\title{
Theory of plasma transport in toroidal confinement systems
}

\author{
F. L. Hinton and R. D. Hazeltine \\ Fusion Research Center, University of Texas at Austin, Austin, Texas 78712
}

The dissipation induced by coulomb-collisional scattering provides an irreducible minimum, and thus a useful standard for comparison, for transport processes in a hot, magnetically confined plasma. The kinetic description of this dissipation is provided by an equation of the Fokker-Planck form. As in the standard transport theory for a neutral gas, approximate solution of the Fokker-Planck equation permits the calculation of transport coefficients, which linearly relate the fluxes of particles, energy, and electric charge, to the density and temperature gradients, and to the electric field. The transport relations are useful in studying the confinement properties of present and future experimental devices for research in controlled thermonuclear fusion. The transport theory for a magnetized plasma (in which the Larmor radius is much smaller than gradient scale lengths describing the plasma fluid) departs from the theory for a neutral gas in several fundamental ways. Thus, transport coefficients for a magnetized plasma can be calculated even when the collisional mean free path is much longer than the gradient scale length (as would pertain in thermonuclear regimes). Such transport coefficients are generally nonlocal, being defined in terms of averages over surfaces with macroscopic dimensions. Furthermore, when the mean free path is long, the magnetized-plasma transport coefficients depend crucially upon the magnetic field geometry, the effects of which must be treated at the kinetic level of the Fokker-Planck equation. The results display several novel couplings between collisional dissipation and the electromagnetic field. The present review of magnetizedplasma transport theory is intended to be as widely accessible as possible. Thus the relevant features of magnetic confinement in closed (toroidal) systems, and of charged particles in spatially varying fields, are derived, at least in outline, from first principles. Although consideration is given to "classical" transport in which most field geometric effects are omitted, major emphasis is placed on the "neoclassical" theory which has been developed over the last decade. Neoclassical transport coefficients are specifically relevant to a magnetically confined plasma, rather than to just a magnetized plasma; their unusual features, such as nonlocality and geometry dependence, become particularly important in the high temperature regime of proposed thermonuclear reactors. The area of neoclassical theory which seems most complete-its application to axisymmetric tokamak-type confinement systems-is correspondingly stressed.

\section{CONTENTS}

I. Introduction

A. History

B. Synopsis

C. Collision operator

D. Conservation laws

E. Small mass-ratio approximations

II. 'Moment Equations

A. Definitions

B. Small gyroradius ordering

1. Refinements

2. Lowest-order consequences

C. Fir st-order equilibrium

1. Confinement geometry

a. Flux coordinates

b. Poloidal and toroidal fields

c. Flux-surface average

2. Equilibrium flow

3. First-order stress

D. Second-order fluxes

1. Classical diffusion

2. Neoclassical transport

E. Axisymmetric systems

1. Ampere's law

2. Second-order fluxes

3. Faraday's law

F. Entropy and heat production

III. Guiding Center Equations

A. Basic formalism

B. Drift surfaces

C. Axisymmetric systems

D. Diffusion

1. Collisional regime

2. Collisionless regimes

3. Asymmetry

E. Distribution function

1. Velocity coordinates

2. Gyrophase dependence
3. Drift-kinetic equation

260

261

261

A. Introduction

B. Classical perpendicular transport

1. Particle fluxes

2. Energy fluxes

C. Parallel transport

1. Kinetic equation

2. Electron version

3. Ion version

4. Variational principle

D. Pfirsch-Schlüter transport

1. Radial fluxes

2. Neoclassical transport coefficients

V. Kinetic Theory of Transport in Axisymmetric Systems

A. Small gyroradius expansion

1. Electron-ion collision term

2. Ion-electron collision term

3. Maximal ordering

4. Perturbation expansion

5. Second-order equations

B. Linearized kinetic equations

1. Coordinate system

2. Transformation of the kinetic equations 273

3. Transport coefficients

4. Weak-coupling approximation $\quad 275$

5. Two ion-species problem 276

C. General variational principle 276

D. Banana regime 278

1. Definitions 278

2. Electron distribution function 278

3. Variational principle 279

4. Lorentz model 280

5. Ion distribution function 282

E. Collisional regime 282

1. Introduction 282

2. Electron transport 282 
3. Ion transport

VI. Transport Coefficients for Large Aspect-Ratio Tokamaks

A. Geometry

B. Electron transport in the banana regime

1. Large aspect-ratio expansion

2. Effects of electron-electron collisions

C. Ion transport in the banana regime

1. Ion thermal conductivity

2. Ion parallel flow

D. The banana-plateau transition

1. The plateau limit

a. Electron transport

b. Ion transport

2. Variational calculation

3. Boundary layer calculation

4. Lorentz model

E. The plateau-collisional transition

1. Electron transport

2. Ion transport

3. Model like-particle collision operator

F. Summary of results

1. Electron fluxes

2. Ion fluxes

3. Weak-coupling approximation

4. Two ion species problem

VII. Closure of the Moment Equations

\section{A. Faraday's law}

B. Ampere's law

C. Transport relations

D. Particle conservation law

E. Energy conservation laws

F. Adiabatic compression

G. Summary

Acknowledgments

References

\section{INTRODUCTION}

A principal object of the controlled fusion program (Post, 1956) is the containment of a thermonuclear plasma by means of strong magnetic fields. Here, containment entails maintaining plasma temperatures in the neighborhood of $10^{9} \mathrm{~K}$ for a time-depending upon the density of plasma ions-long enough for appreciable fusion reactions to occur. Hence the basic role of the magnetic field is to provide thermal isolation between the plasma and the walls of the reactor vessel.

Since experiments with magnetically confined plasma began in the 1950's, a major obstacle in the way of containment has come from plasma instability, manifested either through violent bulk distortions of the discharge column or through strong, fine-scale turbulence. However, during the last decade, and especially since the introduction of the Tokamak confinement device (Artsimovich, 1972), the most serious instability problems appear to have been ameliorated. One result has been a renewed interest in the fundamentally unavoidable mechanisms for loss of containment: diffusion and heat conduction across the confining magnetic field, due to the thermal fluctuations present in even a perfectly stable and quiescent plasma. The effects of such fluctuations are described by a collision operator of the Fokker-Planck form, which will be considered presently. We use the term collisional transport in reference to the resulting transport processes (of course the word "collision" requires interpretation when applied to the shielded Coulomb interaction between particles in a plasma).

This review is concerned with collisional transport in a magnetized plasma, that is, a plasma in which the Larmor frequency, describing charged particle gyration around magnetic field lines, is much larger than any other characteristic frequency of interest. Since each charged particle in such a plasma is "tied" to the field line about which it gyrates, diffusion across the magnetic field is possible only because of collisions, which allow particles to migrate from one field line to another. Therefore, the diffusion rate in a magnetized plasma is typically an increasing function of the collision frequency (rather than a decreasing function, as in the case of a neutral gas). Since magnetic inhibition of free streaming is effective only in directions perpendicular to the field lines, containment of a quasi-equilibrium plasma, for times long enough for diffusion to be a significant process, generally requires a toroidal confinement system.

It has long been recognized that collisional transport is too slow to offer a serious impediment to controlled fusion, especially in view of the large dimensions typical of proposed fusion reactors. This is mainly because the Coulomb-collision frequency varies as the inverse cube of a typical particle speed; at thermonuclear temperatures, collisional effects become quite weak. Furthermore, there are compelling experimental and theoretical indications that "anomalous" transport processes (resulting, for example, from microscopic plasma turbulence) have, and may continue to have, a dominant effect. The study of purely collisional transport remains of interest, however, for the following reasons:

(i) Collisional transport predictions provide a standard, against which both the observed diffusion, and the theoretically predicted anomalous transport processes, can instructively be measured.

(ii) Recent theoretical calculations yield collisional transport coefficients which are surprisingly large (orders of magnitude larger than earlier predictions), which depend upon the magnetic field configuration in a previously unexpected manner, and which imply novel couplings between diffusion and the electromagnetic field. These recent results are intrinsically interesting.

(iii) The transport theory for a magnetically confined plasma represents a qualitative and fundamental departure from "classical" theories, such as the ChapmanEnskog theory for a neutral gas (Chapman and Cowling, 1952). In particular, only the former applies to the case in which the mean free path is longer than a characteristic dimension of the system. The resulting transport equations are nonlocal: transport coefficients are defined in terms of integrals over certain surfaces, determined by the field geometry, which have dimensions comparable to those of the confining system. The resemblance between such a global transport theory and the Chapman-Enskog theory is therefore somewhat superficial. Yet the former also yields a closed set of equations, which determines the time evolution of the ion or electron density and temperature on each appro- 
priately defined surface, even as, in response to transport, the surface configuration changes in time.

It is useful to distinguish two phases in the development of magnetized-plasma transport theory. In the earlier, classical phase, the appropriate form of the Fokker-Planck collision operator was established, and then used to analyze transport properties both parallel and perpendicular to the magnetic field. It is a characteristic of classical transport theory that the spatial variation of the magnetic field has no effect, at least at the level of the kinetic equation; classical transport coefficients can be, and usually were, calculated for the case of a uniform field. The second phase of development, which is now generally referred to as neoclassical, began in the 1960's, especially after the pioneering work of Galeev and Sagdeev (1968). Neoclassical transport theory is also based on the use of the FokkerPlanck collision operator; it departs from classical theory in recognizing that the magnetic field of a toroidal confinement system is necessarily nonuniform, and that in the long mean-free-path regime appropriate to thermonuclear temperatures, the spatial variation of the field has crucial effects, which must be treated at the kinetic level.

The crucial effects of field variation are particle trapping and the gradient- $B$ and curvature drifts of gyrating particles across field lines, resulting from gradual distortion of the Larmor orbit. Thus the magnetic inhibition of free particle motion, mentioned previously, has complementary weakness. Because it provides localization of charged particles in only two dimensions, it requires a toroidal system for containment; but the field curvature resulting from toroidicity leads to perpendicular drifts, i.e., to a weakening of the original inhibition. Neoclassical transport is a prominent result of these weaknesses. It should be noted that, while classical and neoclassical transport processes are additive, neoclassical effects are typically much larger; recall the previous remark (ii).

The present review considers classical theory only briefly, for purposes of comparison and for completeness. A relatively comprehensive treatment of neoclassical theory is attempted. We have tried to make the argument self-contained, and accessible to as wide an audience as possible, including researchers unfamiliar with plasma transport theory. Especially for the benefit of the latter, we draw attention to the following previous reviews.

The famous monograph of Spitzer (1967) discusses, among other relevant topics, the nature of Coulomb collisions in a plasma, and a number of classical transport processes. More detailed treatments of the classical theory, with extensive references, may be found in Braginskii (1965) and Kaufman (1966). The microscopic processes underlying neoclassical transport, as related to field geometry, are considered by Kadomtsev and Pogutse (1971), who also discuss a related class of plasma instabilities. Neoclassical transport is also considered in a review by Galeev and Sagdeev (1975), and in a relatively elementary review by Hazeltine (1975). Finally, a coherent-if unfortunately somewhat dated-account of the experimental observations on to- roidal plasma confinement has been presented by Artsimovich (1972).

\section{A. History}

In less than ten years, the theory of neoclassical transport has acquired a vast literature. We survey the major contributions here, assuming (conveniently, but only temporarily) that the reader is familiar with neoclassical terminology.

The kinetic theory of plasma transport in tokamaks was initiated by Galeev and Sagdeev (1968). They showed that trapped particles, with "banana" orbits, are responsible for a significant enhancement of the calculated diffusion coefficient and thermal conductivity, at very small values of collision frequency (i.e., in the banana regime). ${ }^{1}$ The enhancement was explained qualitatively on the basis of a random walk of the banana orbits, with a step size significantly larger than the Larmor radius. Galeev and Sagdeev also demonstrated the existence of an intermediate collision frequency regime, in which the diffusion coefficient is independent of collision frequency (the "plateau regime").

Plasma diffusion in a stellerator was investigated by Galeev, Sagdeev, Furth, and Rosenbluth (1969). They found a maximum in the diffusion coefficient at low collision frequencies, due to the existence of "localized" particles (Gibson and Taylor, 1967; Gibson and Mason, 1968), with "superbanana" orbits.

The neoclassical theory of plasma transport generated considerable interest, because of its novel character and because of its possible importance in determining toroidal confinement properties. Kovrizhnykh (1969) and Rutherford (1970) independently pointed out that the ion and electron diffusion rates should be equal and independent of the radial electric field, in axisymmetric systems, such as tokamaks. Kovrizhnykh calculated the transport coefficients for a variety of confinement devices (tokamak, levitron, stellerator, and bumpy torus), and considered both weakly ionized and fully ionized plasmas. The details of the calculations, for axisymmetric systems, are particularly clear in Rutherford's paper. Frieman (1970) considered diffusion in general nonaxisymmetric systems. His paper contains a derivation of the drift kinetic equation, which was the starting point for the Galeev-Sagdeev calculation.

Other neoclassical transport properties were also soon discovered. The effect of trapped electrons on the Ohmic conductivity of a tokamak plasma, was considered by Hinton and Oberman (1969) who showed that, at low collision frequencies, the conductivity is reduced by an amount proportional to the fraction of trapped electrons. The possibility of a new kind of pinch effect, due to trapped particles, was discovered independently by Ware (1970) and Galeev (1971). They pointed out that the toroidal electric field, which is necessarily present in tokamaks, causes a radially inward motion of trapped particles, at a velocity which is much larger than the

\footnotetext{
${ }^{1}$ The orders of magnitude of these coefficients in the high collision frequency regime had been worked out several years earlier by Pfirsch and Schlütter (1962) and Shafranov (1965) from a fluid model.
} 
$E \times B$ drift in the classical pinch effect. The existence of a "diffusion driven" current ("bootstrap current") was predicted independently by Galeev (1971) [see also Sagdeev and Galeev (1970), and Galeev and Sagdeev (1971)] and Bickerton, Connor and Taylor (1971). The latter authors considered the possibility of a steadystate "bootstrap" tokamak, in which the poloidal confining magnetic field would be provided by this "bootstrap" current, and in which the toroidal electric field would be zero; this possibility was also considered independently by Kadomtsev and Shafranov (1972). The physical origin of the bootstrap current is the banana orbits of the trapped electrons, in the presence of a density gradient. These result in a current along the magnetic field lines, analogous to the classical diamagnetic current due to particle gyration about the field lines. Through collisions with ions, these particles become untrapped; through collisions with untrapped electrons, the latter are "entrained." The result of these collisions is a current carried by untrapped electrons.

All of the calculations referred to used either simplified collision operators to describe like-particle collisions, or used nonrigorous approximations to deal with the exact Fokker-Planck operators. The resulting numerical transport coefficients differed by as much as an order of magnitude from one paper to the next. Rosenbluth, Hazeltine and Hinton (1972) carried out an accurate and systematic evaluation of the tokamak neoclassical coefficients, starting with the full Fokker-Planck collision operator. By using a variational principle to treat the low collision frequency banana regime, the effects of like-particle collisions were included rigorously. Their results are correct to lowest order in the inverse aspect ratio, in the large aspect ratio, circular cross section case.

Although the correct transport coefficients were known in the banana regime (Rosenbluth, Hazeltine and Hinton, 1972) and the plateau regime (Galeev, 1971), it was the transition between these regimes which was thought to be more relevant to the tokamak experiments of the late 1960's and early 1970's. Hinton and Rosenbluth (1973) calculated the transport coefficients for a large aspectratio tokamak in the banana-plateau transitional regime. They used numerical methods to make use of generalizations of a variational principle due to Rutherford (1970). The results showed that diffusion and thermal conductivity coefficients increase monotonically with collision frequency, even in the plateau regime.

None of the previous papers answered the question of how the radial electric field is determined in the quasisteady state of ambipolar diffusion in axisymmetric systems. Rosenbluth, Rutherford, Taylor, Frieman, and Kovrizhnykh (1971) studied this question for the banana regime. By going to fourth order in the gyroradius, they obtained an equation which determines the time rate of change of the toroidal plasma angular momentum, in terms of radial diffusion of angular momentum. The radial electric field is thus determined, through its relation to the plasma toroidal angular velocity. This question was also studied for the collisional regime by Hazeltine (1974), and for the plateau and transitional re- gimes by Tsang and Frieman (1975).

The banana regime analysis of Rosenbluth, Hazeltine and Hinton (1972) was generalized to include noncircular cross section tokamaks, such as doublet (Ohkawa, 1968) by Glasser and Thompson (1973). A further generalization, to include all collisionality regimes, was considered by Bernstein (1974). He generalized the variational principles used by Hinton and Rosenbluth (1973) to include arbitrary axisymmetric systems, and introduced a convenient inner-product formalism, which has been adopted in this review.

In trying to present a coherent account of the subject under review, we frequently use arguments quite different from those of the original contributors. Yet we have attempted proper assignment of credit, not only to those authors mentioned in the brief history above, but to many others whose contributions have been significant. To those authors whose work is not included, we offer our apologies; some omissions were necessary, in order that we might explore the chosen topics in some depth.

\section{B. Synopsis}

This review is concerned with the calculation of transport coefficients, from the Fokker-Planck equation, in the small gyroradius limit. The transport coefficients linearly relate the fluxes (of particles, energy, and electric charge) to the thermodynamic forces (the pressure and temperature gradients for each charged particle species in the plasma, and the electric field). When combined with the exact conservation laws for particles and energy, and with Maxwell's equations, the linear transport relations provide a closed set of equations, which predict the temporal evolution of the plasma fluid, from a properly chosen set of initial and boundary conditions.

The Fokker-Planck collision operator is introduced at the end of this section. In Sec. II, we postpone further kinetic analysis to consider the velocity moments of the Fokker-Planck equation. The moment equations permit a relatively straightforward introduction of the small gyroradius parameter (Sec. II.B). Then the toroidal nature of a magnetically confined plasma equilibriumwhich is critical to neoclassical theory - can be established (Sec. II.C). We find that this equilibrium is necessarily dynamic, i.e., it can exist only in the presence of significant, divergence-free, flows of particles and energy. A crucial averaging operator-the so-called flux surface average-which is local with respect to minor toroidal radius, is introduced. We also derive convenient, perspicuous expressions for the cross-field particle and energy fluxes, and compare the classical and neoclassical contributions (Sec. II.D). The changing character of diffusion, for different regimes of collision frequency, is considered. These results are derived for the case of a completely general confinement geometry; simplifications permitted in the axisymmetric case, in which the major toroidal axis is a symmetry axis, are considered separately (Sec. II.E). Finally, the relevant dissipation mechanisms are summarized, by consideration of the heat production rate (Sec. II.F).

As remarked previously, the slow drift motion of gy- 
rating particles-the so-called guiding center driftacross the nonuniform confining field, plays a fundamental role in neoclassical transport. Hence in Sec. III we adopt a microscopic viewpoint to review the relevant conclusions of the guiding center theory. As in Sec. II, an initially general formalism (Secs. III.A, III.B) is later specialized to the important case of axisymmetry (Sec. III.C). Once the character of the collisionless guiding center orbits is established, conventional random walk arguments are used to estimate diffusion coefficients under various circumstances (Sec. III.D). (These arguments provide useful understanding and their conclusions are approximately correct; but it should be noted that the details of the simplified random walk picture are not always borne out by kinetic theory.) Next we consider the predictions of guiding center theory with regard to the single-particle distribution function, $f(\overrightarrow{\mathrm{x}}, \overrightarrow{\mathrm{v}}, t)$, describing the expected density of particles at the point $(\vec{x}, \vec{v})$ in phase space (Sec. III.E). This is shown to consist of two parts, $f=\bar{f}+\widetilde{f}$, with the following properties: $\tilde{f}$ is determined by Larmor gyration, and yields classical transport, while $\bar{f}$ is determined by guiding center motion, as modified by collisions, and yields neoclassical transport. A small gyroradius approximation to the Fokker-Planck equation-the so-called drift kinetic equation-which $\bar{f}$ must satisfy, is derived here.

Having established, in Secs. II and III, precisely which moments of the distribution function are required, we begin in Sec. IV the actual calculation of transport coefficients. The classical perpendicular transport problem is considered first, and solved by straightforwardly combining some of our previous results (Sec. IV.B). We then turn to the problem of collisional transport parallel to the magnetic field (Sec. IV.C). The variational method used to treat this problem has some resemblance to techniques used in later sections, to which it therefore provides a useful introduction. Furthermore, we show that the parallel transport equations are readily combined with certain conclusions of Sec. II, to yield the neoclassical (cross-field) transport coefficients for an arbitrary toroidal geometry, in the large collision frequency limit (Sec. IV.D).

Unfortunately, under the experimental conditions typical of most present and planned toroidal confinement systems, these short mean-free-path results pertain to only a small fraction of the plasma volume. To treat a less collision-dominated plasma (including, in particular, a thermonuclear plasma), more sophisticated kinetic theory, based on the drift kinetic equation, is required. This theory, specialized to the case of an axisymmetric system, is presented in Sec. V. First we present a refined, and slightly modified, version of the small gyroradius ordering of Sec. II. In the refined version, the electron to ion mass ratio is ordered with respect to the gyroradius, in such a way as to allow the maximum number of transport effects to be considered simultaneously (Sec. V.A). The ordering yields an expansion of the drift-kinetic equation in powers of the (poloidal) gyroradius. The expansion is treated slightly unconventionally here (Sec. V.B), in order to unify as much as possible the analysis of two transport problems: that of a pure plasma, with electrons and one species of ion, and that of a plasma with several ion species. The linearized kinetic equations so obtained are novel also in their more general and rigorous treatment of certain ion-electron coupling effects. Next, we show that the kinetic equations can be obtained from a variational principle, which is valid independently of the size of the collision frequency, i.e., in both short and long mean-free-path regimes (Sec. V.C). As a result, variational expressions for the transport coefficients are obtained, in terms of integrals of the distribution function. A simpler variational principle is derived for the long mean-free-path limit (Sec. V.D). In the opposite, collision-dominated limit, the kinetic equations can be solved directly (Sec. V.E); one thus obtains, by a somewhat different argument, the axisymmetric version of the results of Sec. IV.D.

In long mean-free-path regimes, the linearized kinetic equations involve a quite complicated average of the already formidable Fokker-Planck collision operator, so that rigorous solutions have been obtained only in certain limits. For example, if the ionic charge $Z$ is very large, the electron collision operator reduces to a Lorentz gas operator (to lowest order in $Z^{-1}$ ), and the kinetic theory is analytically tractable; this case is considered in Sec. V.D. A more generally important limit is that of a "thin" (large aspect-ratio) torus, whose minor radius is much smaller than its major radius. This case is considered in Sec. VI; we show that, for sufficiently small collision frequency, the distribution function is localized in velocity space, in such a way that the collision operator may be expanded in powers of the square root of the inverse aspect ratio. Since the lowest-order version resembles a Lorentz gas operator, the kinetic equations are readily solved. The velocity space region in which $\bar{f}$ is localized changes as the collision frequency increases (while remaining smaller than it is in the collision-dominated regime, where $\bar{f}$ is not localized). Hence somewhat different analytical treatments are required, for the nearly collisionless limit (Secs. VI.B, VI.C), and for the regime of intermediate collisionality (Sec. VI.D). Results for the intermediate regime are affected by the behaviour of $\bar{f}$ near a certain boundary layer in velocity space. Explicit neoclassical transport coefficients, which are rigorous in the context of the aspect-ratio expansion, are obtained for the low-to-intermediate transitional regime by substituting numerical solutions to the large aspect-ratio kinetic equations into the general variational formalism of Sec. V.

For the regime of intermediate to high collisionality (Sec. VI.E) transport coefficients are obtained by numerical solution of the large aspect-ratio kinetic equations. Approximate, but very convenient, interpolation formulas are then used to connect smoothly the numerical values of the coefficients in the various collision frequency regimes. The final expressions for the transport coefficients may be found at the end of Sec. VI (Sec. VI.F).

In Sec. VII we return our attention to the moment equations, which express the conservation of particles and energy for each plasma species. The flux-surface averages of these equations, describing transport in minor radius, are seen to involve the particle and energy fluxes, and the electric current, in precisely the same forms as were calculated from transport theory in Secs. 
IV-VI. Then, to obtain a closed set of equations for the plasma fluid, the conservation laws and transport relations are combined with Maxwell's equations. The electromagnetic coupling is especially interesting, because the explicit form of the flux-surface average depends upon the magnetic field configuration, which can change, in general, on the same time scale as other processes of interest. Thus the global nature of neoclassical transport profoundly affects the problem of closing the set of moment equations. The closed set of equations derived here differs from previously published versions, and is intended to be more generally convenient. However, the closure problem is treated only in axisymmetric geometry.

An exhaustive treatment of neoclassical transport theory is not attempted in this review. In particular, we consider only briefly the large literature concerning transport in asymmetrical confinement systems, such as the stellerator. [Early work on transport in stellerators is reviewed by Galeev and Sagdeev (1975).] Transport coefficients for the asymmetrical case are presented, in terms of averages involving the field geometry, only in the short mean-free-path regime. Our discussion of rotational relaxation is similarly incomplete, and several topics relevant to a contaminated plasma, such as charge exchange effects, are barely mentioned. Most of the omitted topics relate, in our opinion, to elements of the theory which, although potentially very important, have yet to be thoroughly and rigorously developed.

Although the axisymmetric theory is developed, as much as possible, in the context of arbitrary magnetic geometry, this is mainly to emphasize the fact that the transport theory does not depend upon any specific assumptions concerning the magnetic field configuration. Explicit numerical results are thus given only for the large aspect ratio, circular cross section case, which is most generally useful.

\section{Collision operator}

All of the transport literature under review is based on the assumption that the distribution function $f_{a}(\overrightarrow{\mathrm{x}}, \overrightarrow{\mathrm{v}}, t)$ satisfies the Fokker-Planck equation,

$$
\partial f_{a} / \partial t+\overrightarrow{\mathrm{v}} \cdot \vec{\nabla} f_{a}+\left(e_{a} / m_{a}\right)\left(\overrightarrow{\mathrm{E}}+c^{-1} \overrightarrow{\mathrm{v}} \times \overrightarrow{\mathrm{B}}\right) \cdot \partial f_{a} / \partial \overrightarrow{\mathrm{v}}=C_{a}(f),
$$

where $e_{a}$ is the charge and $m_{a}$ the mass of particles of species $a, \overrightarrow{\mathrm{E}}$ and $\overrightarrow{\mathrm{B}}$ are, respectively, the (macroscopic) electric and magnetic fields, and $C_{a}$ is the FokkerPlanck collision operator:

$$
\begin{aligned}
& C_{a}=\sum_{b} C_{a b}, \\
& C_{a b}=-\frac{2 \pi e_{a}^{2} e_{b}^{2}}{m_{a}} \ln \Lambda \frac{\partial}{\partial v_{\alpha}} \int d^{3} v^{\prime} {\left[\frac{f_{a}(v)}{m_{b}} \frac{\partial f_{b}\left(\overrightarrow{\mathrm{v}}^{\prime}\right)}{\partial v_{\beta}^{\prime}}\right.} \\
&\left.-\frac{f_{b}\left(v^{\prime}\right)}{m_{a}} \frac{\partial f_{a}(\overrightarrow{\mathrm{v}})}{\partial v_{\beta}}\right] U_{\alpha \beta}\left(\overrightarrow{\mathrm{v}}-\overrightarrow{\mathrm{v}}^{\prime}\right) .
\end{aligned}
$$

Here, a sum over repeated Cartesian indices $(\alpha, \beta)$ is implied, and

$$
U_{\alpha \beta}(\overrightarrow{\mathrm{x}}) \equiv x^{-3}\left(x^{2} \delta_{\alpha \beta}-x_{\alpha} x_{\beta}\right) .
$$

The Coulomb logarithm is formally given by

$$
\ln \Lambda=\ln (9 N),
$$

where $N \gg 1$ is the number of particles in a sphere of radius $\lambda_{D}$, the Debye length.

Equation (1.2) was first derived by Landau (1936). Chandrasekhar (1943) used a different argument to derive a special case of Eq. (1.2), which was later extended, somewhat, by Cohen, Spitzer and Routly (1950). Rosenbluth, MacDonald and Judd (1957) derived the expression (for $e_{a}^{2}=e_{b}^{2}$ )

$$
C_{a}=-\Gamma_{a}\left[\frac{\partial}{\partial v_{\alpha}}\left(f_{a} \frac{\partial h_{a}}{\partial v_{\alpha}}\right)-\frac{1}{2} \frac{\partial^{2}}{\partial v_{\alpha} \partial v_{\beta}}\left(f_{a} \frac{\partial^{2} g}{\partial v_{\alpha} \partial v_{\beta}}\right)\right],
$$

where $\Gamma_{a}=4 \pi e^{4} \ln \Lambda / m_{a}^{2}$, and

$$
\begin{aligned}
& h_{a}=\sum_{b}\left(1+m_{a} / m_{b}\right) \int d^{3} \overrightarrow{\mathrm{v}}^{\prime} f_{b}\left(\overrightarrow{\mathrm{v}}^{\prime}\right) /\left|\overrightarrow{\mathrm{v}}-\overrightarrow{\mathrm{v}}^{\prime}\right|, \\
& g=\sum_{b} \int d^{3} \overrightarrow{\mathrm{v}}^{\prime} f_{b}\left(\overrightarrow{\mathrm{v}}^{\prime}\right)\left|\overrightarrow{\mathrm{v}}-\overrightarrow{\mathrm{v}}^{\prime}\right| .
\end{aligned}
$$

It is not hard to show that Eqs. (1.2) and (1.5) are equivalent; for many applications, the latter is more convenient. The functions $h_{a}$ and $g$ are called "Rosenbluth potentials," because they satisfy the differential equations

$$
\begin{aligned}
& \left(\nabla_{v}\right)^{4} g=-8 \pi \sum_{b} f_{b}, \\
& \left(\nabla_{v}\right)^{2} h_{a}=-4 \pi \sum_{b}\left(1+m_{a} / m_{b}\right) f_{b},
\end{aligned}
$$

where $\left(\nabla_{v}\right)^{2}=\partial^{2} / \partial v_{\alpha} \partial v_{\alpha}$, etc.

We review neither the derivations of Eq. (1.2) nor the attempts to derive improved operators [see the review by Fried (1966)]. However, some comments on the validity of the Fokker-Planck formalism are appropriate.

A basic element in all derivations of the collision operator is that, because of the long-range nature of the Coulomb interaction, the great majority of scattering events result in quite small deflections: $\Delta \vec{v} / v \ll 1$. In the absence of coherent wave propagation, the effect of many such deflections is to cause each particle to perform a random walk in velocity space; the corresponding evolution of the velocity distribution function is then determined by an operator having the Fokker-Planck form of Eq. (1.5).

Because the range of the "bare" Coulomb force is infinite, the accumulated effect of very small deflections is divergent, unless Debye shielding is taken into account. In its simplest form, this shielding-the result of space-charge polarization-appears in the Coulomb logarithm: the Coulomb interaction is cut off at a distance corresponding to the Debye length. Thus a more general expression for the Coulomb logarithm is given by (Spitzer, 1967; Braginskii, 1965)

$$
\ln \Lambda \equiv \ln \left(b_{\max } / b_{\min }\right),
$$

where $b_{\max }$ and $b_{\min }$ are, respectively, the largest and smallest values of the impact parameter for the class of 
scattering processes under consideration. Equation (1.4) is recovered by choosing $b_{\max }$ to be the Debye length and $b_{\min }$ to be the classical distance of closest approach, for a particle moving at the thermal speed. This choice is suitable if the minimum deflection, caused by collisions at impact parameter $b_{\max }$, exceeds the uncertainty in velocity implied by quantum mechanics (Marshak, 1941). Thus quantum effects impose on $b_{\max }$ an upper limit, which becomes relevant for the case of electron scattering in a hot plasma. An expression for the Coulomb logarithm which includes these effects is given by (Braginskii, 1965)

$$
\begin{aligned}
& \ln \Lambda=23.4-1.15 \log n+3.45 \log T_{e}, \text { for } T_{e}<50 \mathrm{eV} ; \\
& \ln \Lambda=25.3-1.15 \log n+2.3 \log T_{e}, \quad \text { for } T_{e}>50 \mathrm{eV} .
\end{aligned}
$$

Here $n$ is the density in $(\mathrm{cm})^{-3}$, and $T_{e}$ is the electron temperature measured in electron volts.

The cutoff procedure as a whole can be justified only if the plasma is quiescent (i.e., microscopically stable) and if the Coulomb logarithm is large. For most plasmas of thermonuclear interest, $\ln \Lambda$ is between 15 and 20.

Of course, the Fokker-Planck operator cannot accurately describe the effects of large-angle deflections (which are relatively rare, in so far as $\ln \Lambda \gg 1$ ). Operators which include large-angle scattering terms, and which require for their validity only $\Lambda \gg 1$, have been constructed; this topic was reviewed by Kihara and Aono (1971).

Finally, the derivation of the Fokker-Planck equation assumes that in the absence of Coulomb interaction, particle trajectories are straight. When the magnetic field is so strong that the gyrofrequency, $\Omega$, is comparable to the plasma frequency, $\omega_{p}$, as can occur in some confinement devices of interest, this assumption is not strictly justified. The modification of the collision operator which is required to treat the case $\Omega \gg \omega_{p}$ has been considered [see, for example, Montgomery, et al. (1974), and references cited therein]; it appears that a dominant effect is to replace the Debye length cutoff by a gyroradius cut-off, in the Coulomb logarithm.

\section{Conservation laws}

A realistic collision operator must conserve particles, momentum, and energy. These conservation laws are expressed by the following relations between moments of $C_{a b}$ :

$$
\begin{aligned}
& \int d^{3} \overrightarrow{\mathrm{v}} C_{a b}=0, \\
& \int d^{3} \overrightarrow{\mathrm{v}}\left[m_{a} \overrightarrow{\mathrm{v}} C_{a b}+m_{b} \overrightarrow{\mathrm{v}} C_{b a}\right]=0, \\
& \int d^{3} \overrightarrow{\mathrm{v}}\left[m_{a} v^{2} C_{a b}+m_{b} v^{2} C_{b a}\right]=0 .
\end{aligned}
$$

It is easily verified, by straightforward substitution and integration by parts, that the collision operator of Eq. (1.2) satisfies Eqs. (1.10)-(1.12). Other basic properties of the collision operator (e.g., that it satisfies Boltzmann's $H$ theorem) are verified in later sections.

\section{E. Small mass-ratio approximations}

The unlike-species $(a \neq b)$ collision operator can usually be simplified when the two species have disparate masses. For definiteness, consider first the case of electrons $(a=e)$ being scattered by ions $(b=i)$. The basic simplifying assumption is that $f_{i}$ is strongly peakedconcentrated in a narrow range of velocities-compared to $f_{e}$. It follows in particular that the unlike-species contribution to the Rosenbluth potential $g$ [Eq. (1.7)] may be approximated by

$$
\begin{aligned}
\int d^{3} \overrightarrow{\mathrm{v}}^{\prime} f_{i}\left(\overrightarrow{\mathrm{v}}^{\prime}\right)\left|\overrightarrow{\mathrm{v}}-\overrightarrow{\mathrm{v}}^{\prime}\right| & \simeq \int d^{3} \overrightarrow{\mathrm{v}} f_{i}\left(\overrightarrow{\mathrm{v}}^{\prime}\right) v\left(1-\overrightarrow{\mathrm{v}} \cdot \overrightarrow{\mathrm{v}}^{\prime} / v^{2}\right) \\
& =n_{i} v\left(1-\overrightarrow{\mathrm{u}}_{i} \cdot \overrightarrow{\mathrm{v}} / v^{2}\right),
\end{aligned}
$$

where $n_{i}$ is the ion density, and $\overrightarrow{\mathrm{u}}_{i}$ is the ion flow velocity

$$
n_{i} \overrightarrow{\mathrm{u}}_{i}=\int d^{3} \overrightarrow{\mathrm{v}} \overrightarrow{\mathrm{v}} f_{i}(\overrightarrow{\mathrm{v}})
$$

which has also been assumed small compared to $\vec{v}$, over most of the domain of $f_{e}(\overrightarrow{\mathrm{v}})$. Similarly approximating the ion contribution to Eq. (1.6), and substituting the results into Eq. (1.5), we obtain

$C_{e i} \simeq\left(2 \pi e^{4} \ln \Lambda / m_{e}^{2}\right) n_{i}(\partial / \partial \overrightarrow{\mathrm{v}}) \cdot \mathrm{U}\left(\overrightarrow{\mathrm{v}}-\overrightarrow{\mathrm{u}}_{i}\right) \cdot\left(\partial f_{e} / \partial \overrightarrow{\mathrm{v}}\right)$.

Here, terms of second- or higher-order in $\left|u_{i} / v\right|$ are to be neglected.

These approximations can be made more explicit if we assume that both distribution functions are nearly Maxwellian

$$
f_{a}=f_{a M}\left(1+\hat{f_{a}}\right),
$$

where

$$
f_{a M}=\pi^{-3 / 2} v_{\text {tha }}^{-3} n_{a} \exp \left(-v^{2} / v_{\text {th } a}^{2}\right),
$$

with $v_{\text {tha }}$ being the thermal speed and

$$
\left|\hat{f}_{a}\right| \ll 1 \text {. }
$$

If the temperatures of the two species are roughly comparable, and if $u_{i}$ is not much larger than $v_{\text {thi }}$, then the ratio

$$
v_{\text {thi }} / v_{\text {the }} \sim\left(m_{e} / m_{i}\right)^{1 / 2} \ll 1
$$

provides a natural small parameter, with respect to which Eq. (1.13) represents a first order expansion. The linearized electron-ion collision operator is obtained by assuming $u_{i} / v_{\text {th } i} \sim \hat{f}$, and retaining only first order, $O(\hat{f})$, terms in Éq. (1.14). We find (Braginskii, 1965)

$$
C_{e i}^{l}=\Gamma_{e} n_{i}\left\{\frac{1}{2} \frac{\partial}{\partial \overrightarrow{\vec{v}}} \cdot \mathrm{U}(\overrightarrow{\mathrm{v}}) \cdot \frac{\partial f_{e}}{\partial \overrightarrow{\mathrm{v}}}+\frac{2 \overrightarrow{\mathrm{u}}_{i} \cdot \overrightarrow{\mathrm{v}}}{v^{3} v_{\text {the }}^{2}} f_{e M}\right\} .
$$

Here the $l$ superscript refers to the linearization.

The first term of Eq. (1.16), which involves only the electron distribution function, describes angular scattering at fixed $|v|$, and vanishes if $f_{e}$ is isotropic. This is because, in collisions with the much more massive ions, the energy change of an electron is very small (of order $\left.m_{e} / m_{i}\right)$. Thus neither Eq. (1.14) nor Eq. (1.16) is sufficiently accurate for calculating the rate of energy exchange between the two species.

Momentum exchange between ions and electrons is 
relatively fast, and Eq. (1.16) is applicable. The momentum exchange rate is measured by the electron-ion "friction force,"

$$
\overrightarrow{\mathrm{F}}_{e} \equiv \int d^{3} \dot{\overrightarrow{\mathrm{v}}} m_{e} \overrightarrow{\mathrm{v}} C_{e i}
$$

After straightforward partial integration, Eq. (1.16) yields

$$
\overrightarrow{\mathrm{F}}_{e}=-\Gamma_{e} n_{i} m_{e} \int d^{3} \overrightarrow{\mathrm{v}} f_{e} \overrightarrow{\mathrm{v}} / v^{3}+\left(m_{e} n_{e} / \tau_{e}\right) \overrightarrow{\mathrm{u}}_{i} .
$$

Here

$$
\tau_{e}=3 \pi^{1 / 2} v_{\text {the }}^{3} / 4 n_{i} \Gamma_{e}
$$

provides a convenient measure (which becomes precise for a Maxwellian $f_{e}$ ) of the momentum exchange time. Because typical relaxation processes associated with electron-electron collisions also proceed at the rate $\tau_{e}^{-1} . \quad \tau_{e}$ is often called the electron collision time.

The small mass-ratio approximation to the ion-electron collision operator, $C_{i e}$, may be obtained in a similar way. We again assume $f_{e}$ has the nearly Maxwellian form of Eq. (1.15), and neglect $\hat{f}_{e}$ in calculating $g$ from Eq. (1.7). However, the contribution of $\hat{f}_{e}$ to $h_{i}$ [Eq. (1.6) ] can be comparable, formally, to other terms in $C_{i e}$, so we must compute

$$
\partial h_{i}\left(\hat{f_{e}}\right) / \partial \overrightarrow{\mathrm{v}} \cong-\left(m_{i} / m_{e}\right) \int d^{3} \overrightarrow{\mathrm{v}}^{\prime} f_{\theta M}\left(\overrightarrow{\mathrm{v}}^{\prime}\right) f_{e}\left(\overrightarrow{\mathrm{v}}^{\prime}\right) \overrightarrow{\mathrm{v}}^{\prime}\left(v^{\prime}\right)^{-3} .
$$

Here we have retained only the dominant term in $\left|\vec{v}^{\prime}-\vec{v}\right|^{-1}$. Equation (1.17) provides a relation $\partial h_{i}\left(\hat{f}_{e}\right) / \partial \vec{v}$ $=m_{i} \overrightarrow{\mathrm{F}}_{e} / \Gamma_{e} n_{i} m_{e}^{2}-m_{i} n_{e} \overrightarrow{\mathrm{u}}_{i} / \Gamma_{e} n_{i} \tau_{e} m_{e}$. Hence, after straightforwardly evaluating $g\left(f_{e M}\right)$ and $h_{i}\left(f_{e M}\right)$, we obtain the collision operator

$$
C_{i e}=\frac{m_{e}}{m_{i}} \frac{n_{e}}{n_{i} \tau_{e}} \frac{\partial}{\partial \overrightarrow{\mathrm{v}}} \cdot\left[\frac{T_{e}}{m_{i}} \frac{\partial f_{i}}{\partial \overrightarrow{\mathrm{v}}}+\left(\overrightarrow{\mathrm{v}}-\overrightarrow{\mathrm{u}}_{i}\right) f_{i}\right]+\frac{1}{m_{i} n_{i}} \overrightarrow{\mathrm{F}}_{e} \cdot \frac{\partial f_{i}}{\partial \overrightarrow{\mathrm{v}}},
$$

where $T_{e} \equiv m_{e} v_{\text {the }}^{2} / 2$. In Eq. (1.18), the first term, in square brackets, determines the rate of energy exchange. The last term, involving $\overrightarrow{\mathrm{F}}_{e}$, is responsible for momentum conservation, as in Eq. (1.11).

Finally, it is convenient to present here the form of the like-species, linearized collision operator. Equations (1.2) and (1.15) readily yield

$$
\begin{gathered}
C_{a a}^{l}=\frac{\Gamma_{a}}{2} \frac{\partial}{\partial v_{\alpha}} f_{a M}(\overrightarrow{\mathrm{v}}) \int d^{3} \overrightarrow{\mathrm{v}}^{\prime} f_{a M}\left(\overrightarrow{\mathrm{v}}^{\prime}\right) U_{\alpha \beta}\left(\overrightarrow{\mathrm{v}}-\overrightarrow{\mathrm{v}}^{\prime}\right) \\
\cdot\left[\frac{\partial \hat{f_{a}(\overrightarrow{\mathrm{v}})}}{\partial v_{\beta}}-\frac{\partial \hat{f_{a}\left(\overrightarrow{\mathrm{v}}^{\prime}\right)}}{\partial v_{\beta}^{\prime}}\right] .
\end{gathered}
$$

\section{MOMENT EQUATIONS}

\section{A. Definitions}

To provide a framework for later discussion, we consider here the velocity moments of Eq. (1.1). First we define, omitting species subscripts for convenience, the particle flux

$$
n \overrightarrow{\mathrm{u}}=\int d^{3} \overrightarrow{\mathrm{v}} \overrightarrow{\mathrm{v}} f
$$

the stress tensor

$$
\mathbf{P}=\int d^{3} \overrightarrow{\mathrm{v}} m \overrightarrow{\mathrm{v}} \overrightarrow{\mathrm{v}} f ;
$$

the energy flux

$$
\overrightarrow{\mathrm{Q}}=\int d^{3} \overrightarrow{\mathrm{v}}\left(m v^{2} / 2\right) \overrightarrow{\mathrm{v}} f,
$$

and the energy-weighted stress tensor

$$
\mathbf{R}=\int d^{3} \overrightarrow{\mathrm{v}}\left(m v^{2} / 2\right) \overrightarrow{\mathrm{v}} \overrightarrow{\mathrm{v}} f .
$$

Of course $n=\int d^{3} \overrightarrow{\mathrm{v}} f$ is the single-species density. The stress tensor is to be distinguished from the pressure tensor,

$$
P^{\prime}=\int d^{3} \overrightarrow{\mathrm{v}} m(\overrightarrow{\mathrm{v}}-\overrightarrow{\mathrm{u}})(\overrightarrow{\mathrm{v}}-\overrightarrow{\mathrm{u}}) f
$$

similarly, the energy flux is to be distinguished from the heat flux

$$
\overrightarrow{\mathrm{q}}=\int d^{3} \overrightarrow{\mathrm{v}}(m / 2)(\overrightarrow{\mathrm{v}}-\overrightarrow{\mathrm{u}})^{2}(\overrightarrow{\mathrm{v}}-\overrightarrow{\mathrm{u}}) f .
$$

We measure the temperature in energy units

$$
p=n T=\operatorname{Tr}\{\mathrm{P}\} / 3,
$$

where $p$ is the scalar pressure, and $\mathrm{Tr}$ denotes the trace. Three moments of the Fokker-Planck operator are also needed; these are the collisional momentum exchange, or friction force

$$
\overrightarrow{\mathrm{F}}=\int d^{3} \overrightarrow{\mathrm{v}} m \overrightarrow{\mathrm{v}} C(f) ;
$$

the collisional energy exchange

$$
Q=\int d^{3} \vec{v}(m / 2)(\vec{v}-\vec{u})^{2} C(f) ;
$$

and the collisional change in energy flux

$$
\overrightarrow{\mathrm{G}}=\int d^{3} \overrightarrow{\mathrm{v}}\left(m v^{2} / 2\right) \overrightarrow{\mathrm{v}} C(f) \text {. }
$$

Making explicit the species subscript $a$, we recall that the conservation of momentum and energy in collisions requires

$$
\begin{aligned}
& \sum_{a} \overrightarrow{\mathrm{F}}_{a}=0, \\
& \sum_{a}\left(Q_{a}+\overrightarrow{\mathrm{F}}_{a} \cdot \overrightarrow{\mathrm{u}}_{a}\right)=0 .
\end{aligned}
$$

Now the even $\left(|v|^{0}\right.$ and $\left.v^{2}\right)$ moments of Eq. (1.1), which express the conservation of particles and energy, respectively, may be written as

$$
\begin{aligned}
& \partial n / \partial t+\vec{\nabla} \cdot(n \overrightarrow{\mathrm{u}})=0, \\
& (\partial / \partial t) 3 p / 2+\vec{\nabla} \cdot \overrightarrow{\mathrm{Q}}=Q+\overrightarrow{\mathrm{u}} \cdot(\overrightarrow{\mathrm{F}}+e n \overrightarrow{\mathrm{E}}) .
\end{aligned}
$$

The odd ( $\overrightarrow{\mathrm{v}}$ and $\left.\left|v^{2}\right| \overrightarrow{\mathrm{v}}\right)$ moments, which express the conservation of momentum and of energy flux, respectively, take the form

$$
\begin{aligned}
& (\partial / \partial t) m n \overrightarrow{\mathbf{u}}+\vec{\nabla} \cdot \mathrm{P}-e n\left(\overrightarrow{\mathrm{E}}+c^{-1} \overrightarrow{\mathbf{u}} \times \overrightarrow{\mathrm{B}}\right)=\overrightarrow{\mathbf{F}}, \\
& \begin{aligned}
(\partial / \partial t) \overrightarrow{\mathbf{Q}}+\vec{\nabla} \cdot \mathbf{R}-(3 / 2)(e / m) \overrightarrow{\mathbf{E}} p-(e / m) \overrightarrow{\mathrm{E}} \cdot \mathbf{P} \\
-(e / m c) \overrightarrow{\mathbf{Q}} \times \overrightarrow{\mathrm{B}}=\overrightarrow{\mathrm{G}} .
\end{aligned}
\end{aligned}
$$


Transport theory of a magnetically confined plasma has the principal object of providing a closed set of equations for the time evolution of the densities and pressures of each species in the plasma fluid. Thus only the even moment equations appear in the final closed set. The odd moment equations are useful mainly because they alone involve the magnetic field. Hence an ordering argument based on the strong field limit can be understood at the fluid, rather than kinetic, level only through consideration of Eqs. (2.15) and (2.16).

This strong field, or small gyroradius, ordering is made precise in the following subsection. It is then used, with Eqs. (2.13)-(2.16), to study the confined plasma equilibrium, to provide physical understanding of several transport processes, and to provide expressions for the cross-field fluxes which are significantly more convenient than the definitions of Eqs. (2.1) and (2.3).

\section{B. Small gyroradius ordering}

We denote the scale length for changes in macroscopic parameters, such as the pressure, by

$$
l \cong|\nabla \ln p|^{-1} ;
$$

the thermal speed by

$$
v_{\text {th }} \equiv(2 T / m)^{1 / 2} ;
$$

and the transit frequency by

$$
\omega \equiv v_{\text {th }} / l \text {. }
$$

A convenient measure of a particle gyroradius (Larmor radius) is then

$$
\rho \equiv v_{\text {th }} / \Omega=m c v_{\text {th }} /(e B) .
$$

The basic ordering assumption is that this length is much smaller than $l$ :

$$
\delta \equiv \rho / l=\omega / \Omega \ll 1 .
$$

We further assume

$$
\partial \ln p / \partial t=O\left(\delta^{2} \omega\right)
$$

and

$$
c E /\left(B v_{\text {th }}\right)=O(\delta),
$$

where $E$ is the electric field. Equation (2.20), in which $p$ could be replaced by $n, T$, etc., serves to identify the (diffusion) time scale under consideration. It is assumed that all faster processes have evolved to equilibrium. Equation (2.21), called the drift ordering, serves to rule out certain rapid fluid motions associated with the case $c E /\left(B v_{\text {th }}\right)=\boldsymbol{O}(1)$ (magnetohydrodynamical ordering).

\section{Refinements}

Because of the small electron-to-ion mass ratio

$$
\left(m_{e} / m_{i}\right)<10^{-3} \text {, }
$$

it is occasionally useful to include the species subscripts; we assume

$$
T_{e} \sim T_{i}, l_{e} \sim l_{i},
$$

so that

$$
\delta_{\dot{e}} / \delta_{i} \sim v_{\text {th } i} / v_{\text {the }} \sim\left(m_{e} / m_{i}\right)^{1 / 2} \ll 1 .
$$

One further refinement is of general importance. In low $-\beta$ toroidal confinement systems, the magnetic field can be decomposed into poloidal $\left(\overrightarrow{\mathrm{B}}_{p}\right)$ and toroidal $\left(\overrightarrow{\mathrm{B}}_{T}\right)$ components such that $\overrightarrow{\mathrm{B}}=\overrightarrow{\mathrm{B}}_{p}+\overrightarrow{\mathrm{B}}_{T}$, with, typically

$$
\left|B_{p} / B_{T}\right| \ll 1
$$

as shown in Sec. II.C, below. Hence we introduce the poloidal gyroradius

$$
\rho_{p}=v_{\text {th }} / \Omega_{p}, \quad \Omega_{p}=e B_{p} / m c,
$$

and let

$$
\delta_{p}=\left(B / B_{p}\right) \delta \text {. }
$$

Thus two measures of the gyroradius may be distinguished for each plasma species. We adopt the strongest version of Eq. (2.19)

$$
\delta_{p i} \ll 1,
$$

since this applies to most of the literature under review. The subscripts are frequently omitted for the sake of simplicity. However, we point out that certain stellerator experiments do not satisfy Eq. (2.26), and that it is only marginally satisfied on some present tokamak devices.

\section{Lowest-order consequences}

As shown by kinetic argument in Sec. V, the orderings (2.19)-(2.21) force the distribution functions to be approximately Maxwellian

$$
\begin{aligned}
& f=f_{M}+O(\delta), \\
& f_{M} \equiv n_{0}\left(\pi^{1 / 2} v_{\text {th }}\right)^{-3} \exp \left[-\left(v / v_{\text {th }}\right)^{2}\right] .
\end{aligned}
$$

Hence in lowest order each plasma species is described by a density $n_{0}$ and a temperature $T=m v_{\text {th }}^{2} / 2$. [This circumstance is so crucial to the linear transport processes under consideration that, if it could not be deduced from the orderings, Eq. (2.27) would be assumed to hold anyway.] Some elementary consequences are that

$$
\{n \overrightarrow{\mathrm{u}}, \overrightarrow{\mathrm{Q}}, \overrightarrow{\mathrm{F}},(\mathrm{P}-\mid p),[\mathrm{R}-(5 p T / 2 m) \mid]\}=O(\delta),
$$

where 1 is the unit dyadic. From these results, and from the definitions of Eqs. (2.3) and (2.6), it follows that

$$
\overrightarrow{\mathrm{q}}=[\overrightarrow{\mathrm{Q}}-(5 / 2) p \overrightarrow{\mathrm{u}}][1+O(\delta)] .
$$

Note also that, in view of the slow time variation described by Eq. (2.20), the electric field is predominantly electrostatic

$$
\left(c / B v_{\text {th }}\right)(\overrightarrow{\mathrm{E}}+\vec{\nabla} \Phi)=\boldsymbol{O}\left(\delta^{2}\right),
$$

where $\Phi$ is the electrostatic potential.

We now turn to the odd moments, Eqs. (2.15) and (2.16). Using Eq. (2.29) to identify the zeroth-order terms, we find

$$
\begin{aligned}
& \overrightarrow{\mathrm{B}} \cdot[\vec{\nabla} p+e n \vec{\nabla} \Phi]=O(\delta), \\
& \overrightarrow{\mathrm{B}} \cdot[\vec{\nabla}(p T)+e p \vec{\nabla} \Phi]=O(\delta) .
\end{aligned}
$$

Hence

$$
\begin{aligned}
& \overrightarrow{\mathrm{B}} \cdot \vec{\nabla} T=O(\delta), \\
& \overrightarrow{\mathrm{B}} \cdot \vec{\nabla}[n \exp (-e \Phi / T)]=O(\delta)
\end{aligned}
$$


In combination with the quasi-neutrality condition

$$
\sum_{a} e_{a} n_{a}=0
$$

Eq. (2.33) yields the constraints

$$
\begin{aligned}
& \overrightarrow{\mathrm{B}} \cdot \vec{\nabla} n=O(\delta)=\overrightarrow{\mathrm{B}} \cdot \vec{\nabla} p, \\
& \overrightarrow{\mathrm{B}} \cdot \vec{\nabla} \Phi=O(\delta) .
\end{aligned}
$$

\section{First-order equilibrium}

\section{Confinement geometry}

Equations (2.15) and (2.16) provide the exact formulae

$$
\begin{aligned}
& n \overrightarrow{\mathrm{u}}_{\perp}=(m \Omega)^{-1} \hat{n} \times[\vec{\nabla} \cdot \mathrm{P}-\overrightarrow{\mathrm{F}}-e n \overrightarrow{\mathrm{E}}+m(\partial / \partial t) n \overrightarrow{\mathrm{u}}], \\
& \overrightarrow{\mathrm{Q}}_{\perp}=\Omega^{-1} \hat{n} \times\left[\vec{\nabla} \cdot \mathrm{R}-\overrightarrow{\mathrm{G}}-(e / m) \overrightarrow{\mathrm{E}} \cdot\left(\mathrm{P}-\frac{3}{2} p \mathrm{I}\right)+\partial \overrightarrow{\mathrm{Q}} / \partial t\right],
\end{aligned}
$$

where $\hat{n} \equiv \overrightarrow{\mathrm{B}} / B$ and $\vec{F}_{\perp} \equiv \hat{n} \times(\vec{F} \times \hat{n})$. Notice that, in order to compute these flows through $O\left(\delta^{n}\right)$, we need evaluate the expressions in brackets only through $O\left(\delta^{n-1}\right)$. In particular the perpendicular current

$$
\overrightarrow{\mathrm{J}}_{\perp}=\sum_{a} e_{a} n_{a} \overrightarrow{\mathrm{u}}_{\perp a}
$$

may be computed from Eqs. (2.29) and (2.37); we find, through first order in $\delta$,

$$
\vec{J} \times \overrightarrow{\mathrm{B}}=c \vec{\nabla} P,
$$

where

$$
P=\sum_{a} p_{a}
$$

denotes the total plasma pressure.

Equation (2.40), together with Ampere's law,

$$
\vec{\nabla} \times \vec{B}=(4 \pi / c) \vec{J},
$$

describes the equilibrium configuration of a magnetically confined plasma. This configuration consists in general of a sequence of nested toroids-the magnetic surfaces, or flux surfaces-(almost) each of which is covered ergodically by a single field line. The innermost, degenerate toroid is called the magnetic axis (Kruskal and Kulsrud, 1958).

Grad (1967) has emphasized that the equilibrium relations need not possess solutions in general. The existence of solutions can be guaranteed only for the axisymmetric case and, in an approximate sense, for near axisymmetry. We therefore restrict our attention to configurations in which any asymmetries are sufficiently weak to permit the existence of nested flux surfaces.

\section{a. Flux coordinates}

Figure 1 shows a typical flux surface, the field line from which it is constructed, and the magnetic axis. The directions of variation of so-called "flux coordinates" $(\Psi, \Theta, \zeta)$ are also shown in the figure. The functions $\Psi(\overrightarrow{\mathrm{x}}), \Theta(\overrightarrow{\mathrm{x}})$, and $\zeta(\overrightarrow{\mathrm{x}})$ have the following properties.

(i) $\Psi$, the radial coordinate, is constant on each flux surface.

(ii) $\Theta(\zeta)$, the poloidal (toroidal) angle, increases by
$2 \pi$ upon one traversal of the minor (major) toroidal circumference.

(iii) $\Theta$ and $\zeta$ are chosen such that the ratio $\overrightarrow{\mathrm{B}} \cdot \vec{\nabla} \Theta /$ $\overrightarrow{\mathrm{B}} \cdot \vec{\nabla} \zeta$ depends only on $\Psi$.

(iv) $\Psi, \Theta$ and $\zeta$ are chosen such that the metric determinant

$$
g(\Psi, \Theta, \zeta) \equiv|\vec{\nabla} \Psi \cdot(\vec{\nabla} \Theta \times \vec{\nabla} \zeta)|^{-2}
$$

is independent of $\zeta$

$$
\partial g / \partial \zeta=0 \text {. }
$$

That these properties are compatible has been shown by Hamada (1962) [see also Greene and Johnson (1961)], who imposed the stronger requirement $g=$ constant, instead of Eq. (2.44).

It is convenient to consider here three specific realizations of the radial coordinate $\Psi$. These are: the poloidal flux (divided by $2 \pi$ ) enclosed by a surface on which lies the given point $\vec{x}$

$$
\psi(\overrightarrow{\mathrm{x}}) \equiv(2 \pi)^{-2} \int d^{3} \overrightarrow{\mathrm{x}} \vec{\nabla} \Theta \cdot \overrightarrow{\mathrm{B}} ;
$$

the enclosed toroidal flux

$$
\phi(\overrightarrow{\mathrm{x}}) \equiv(2 \pi)^{-1} \int d^{3} \overrightarrow{\mathrm{x}} \vec{\nabla} \zeta \cdot \overrightarrow{\mathrm{B}} ;
$$

and the enclosed volume

$$
V(\overrightarrow{\mathrm{x}}) \equiv \int d^{3} \overrightarrow{\mathrm{x}}
$$

In each definition, the integration domain is the interior of that flux surface which passes through $\vec{x}$. The fluxes $\psi$ and $\phi$ can be put in the more familiar, although less convenient, form of surface integrals by noting, for example, $\vec{\nabla} \zeta \cdot \vec{B}=\vec{\nabla} \cdot(\zeta \vec{B})$, applying Gauss' theorem, and using the multivalued nature of $\zeta$. We see that the toroidal flux is simply the flux of $\overrightarrow{\mathrm{B}}$ through the surface $S$ shown in Fig. 1.

Each of the quantities $\psi, \phi$, and $V$ provides an allowable radial coordinate, and we shall have occasion to use all three.

\section{b. Poloidal and toroidal fields}

Noting that $\overrightarrow{\mathrm{B}} \cdot \vec{\nabla} \phi=\overrightarrow{\mathrm{B}} \cdot \vec{\nabla} \psi=\vec{\nabla} \cdot \overrightarrow{\mathrm{B}}=0$, and using Eqs. (2.45) and (2.46), one can express $\vec{B}$ in terms of its flux functions

$$
\overrightarrow{\mathrm{B}}=(2 \pi)^{-1} \vec{\nabla} \phi \times \vec{\nabla} \Theta+\vec{\nabla} \zeta \times \vec{\nabla} \psi
$$

Here, the first term is called the toroidal field $\vec{B}_{T}$, and the second term the poloidal field $\vec{B}_{p}$. These fields are tangent to the (not necessarily orthogonal) $\zeta$ and $\Theta$ coordinate lines, respectively. An equivalent expression is

$$
\overrightarrow{\mathrm{B}}_{p}=\vec{\nabla} \zeta \times \vec{\nabla} \psi, \quad \overrightarrow{\mathrm{B}}_{T}=q \vec{\nabla} \psi \times \vec{\nabla} \Theta,
$$

where the quantity

$$
q(\psi) \equiv(2 \pi)^{-1} d \phi / d \psi
$$

is the reciprocal of the rotational transform, and describes the average pitch of the field lines: $q$ is the number (not rational, in general) of toroidal circuits for one complete poloidal circuit, of a field line around the magnetic axis. 
It can be seen that rational values of $q$ correspond to surfaces which are not ergodically covered, the field line reconnecting with itself after several circuits of the torus. The breakdown of the assumed equilibrium conditions on such exceptional surfaces need not be serious. However, small, nearly integral values of $q$ are dangerous, in low- $\beta$ systems, with regard to fluid stability. Hence $q$ is called the safety factor; a stably confined, low- $\beta$ plasma must have (Kruskal, et al., 1958; G. G. Dolgov-Saveliev, et al., 1960)

$$
q>q_{\min } \sim 1 \text {. }
$$

In fact the safety factor is as large as 4 at the maximum radius in present toroidal devices.

The confinement geometry is further characterized by a representative value of $|\nabla \Theta / \nabla \zeta|$, i.e., the ratio of major to minor toroidal circumference. This "aspect ratio" is evidently larger than 1 and, in typical contemporary devices, as large as 5 ,

$$
|\nabla \Theta| /|\nabla \zeta| \gg 1 \text {. }
$$

Hence, as noted previously, the poloidal field is relatively small $\left|B_{p} / B_{T}\right| \ll 1$.

\section{c. Flux-surface average}

Most of the transport studies considered in this review are nonlocal, in the sense that they relate a certain spatial average of each particle flux, energy flux, etc., to the same average of the driving forces. The useful aver aging operation, called the flux-surface average and denoted by \langle\rangle , is defined to satisfy

$$
\langle\overrightarrow{\mathrm{B}} \cdot \vec{\nabla} \mathfrak{F}\rangle=\langle\vec{\nabla} \cdot(\overrightarrow{\mathrm{B}} \mathfrak{F})\rangle=0,
$$

for any function $F(\Psi, \Theta, \zeta)$; and

$$
\langle\bar{F}\rangle=\bar{F} \text {, }
$$

for any function $\bar{F}(\Psi)$ which is constant on flux surfaces. In other words, \langle\rangle is the normalized, radially local, annihilator for $\vec{B} \cdot \vec{\nabla}$. An explicit form, in terms of the volume average over the infinitesimal volume $\Delta V$ between two neighboring flux surfaces, can be inferred from Eq. (2.53)

$$
\begin{aligned}
\langle F\rangle & =\int_{\Delta V} d^{3} \overrightarrow{\mathrm{x}} F / \int_{\Delta V} d^{3} \overrightarrow{\mathrm{x}}, \\
& =(d \Psi / d V) \int(d S /|\nabla \Psi|) \mathcal{F},
\end{aligned}
$$

where $d S$ is the area element on the flux-surface $\Psi$ which can be any radial coordinate.

Using Eq. (2.55) and Gauss' theorem one can show that, for any vector $\overrightarrow{\mathrm{F}}$

$$
\begin{aligned}
\langle\vec{\nabla} \cdot \overrightarrow{\mathrm{F}}\rangle & =(d / d V)\langle\vec{F} \cdot \vec{\nabla} V\rangle, \\
& =(d \Psi / d V)(d / d \Psi)(d V / d \Psi)\langle\overrightarrow{\boldsymbol{F}} \cdot \vec{\nabla} \Psi\rangle .
\end{aligned}
$$

It follows in particular that

$$
\langle\vec{\nabla} \Psi \cdot \vec{\nabla} \times \vec{F}\rangle=\langle\vec{\nabla} \cdot(\vec{F} \times \vec{\nabla} \Psi)\rangle=0 .
$$

\section{Equilibrium flow}

Because of Eqs. (2.32)-(2.36), and the ergodic property of field lines, the densities, temperatures, and electro-

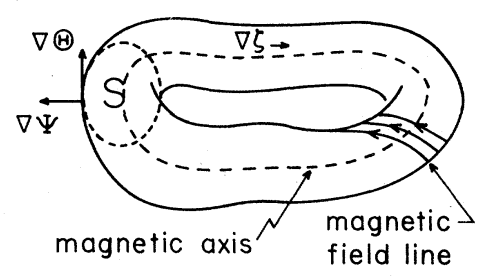

FIG. 1. A toroidal flux surface, showing the directions of variation of the flux coordinates, $\Psi, \Theta$, and $\zeta$. The surface is covered ergodically by a single magnetic field line, a few loops of which are indicated.

static potential are, in lowest order, constant on flux surfaces

$$
\begin{aligned}
& n_{a}=\overline{n_{a}}(\Psi)[1+O(\delta)], \quad T_{a}=\overline{T_{a}}(\Psi)[1+O(\delta)], \\
& \overrightarrow{\mathrm{E}}=-\vec{\nabla} \Phi(\Psi)[1+O(\delta)] .
\end{aligned}
$$

Equations (2.29), (2.30), (2.37), and (2.38) then provide the following expressions for the first order perpendicular flows

$$
\begin{aligned}
& \left(n \overrightarrow{\mathrm{u}}_{\perp}\right)_{1}=(m \Omega)^{-1} \hat{n} \times(\vec{\nabla} \bar{p}+e \bar{n} \vec{\nabla} \Phi), \\
& \overrightarrow{\mathrm{q}}_{\perp_{1}}=(5 / 2)(m \Omega)^{-1} \bar{p} \hat{n} \times \vec{\nabla} \bar{T},
\end{aligned}
$$

where the subscripts refer to the $\delta$ ordering, i.e.,

$$
\left(n \overrightarrow{\mathrm{u}}_{\perp}\right)_{n}=O\left(\delta^{n}\right) \text {. }
$$

These flows, which result from Larmor gyration, evidently remain within the magnetic surface

$$
\left(n \overrightarrow{\mathrm{u}}_{\perp}\right)_{1} \cdot \vec{\nabla} \Psi=\overrightarrow{\mathrm{q}}_{\perp_{1}} \cdot \vec{\nabla} \Psi=0 .
$$

According to Eq. (2.20), the total first-order flows must be divergence free

$$
\vec{\nabla} \cdot(n \overrightarrow{\mathrm{u}})_{1}=\vec{\nabla} \cdot \overrightarrow{\mathrm{q}}_{1}=0 .
$$

Equations (2.61)-(2.64) can be satisfied only in the presence of parallel "return" flows, which serve to maintain the presumed relatively slow variation of $n_{a}$ and $T_{a}$.

In order to derive useful expressions for these parallel motions, we note from Eq. (2.63) that $\left(n \overrightarrow{\mathrm{u}}_{1}\right)$, for example, may be written as

$$
(n \overrightarrow{\mathrm{u}})_{1}=\hat{K} \overrightarrow{\mathrm{B}}+\tilde{K} \vec{\nabla} \psi \times \vec{\nabla} \Theta
$$

for some functions $\hat{K}$ and $\tilde{K}$. Equation (2.64) requires

$$
g^{-1 / 2} \partial \tilde{K} / \partial \zeta=-\overrightarrow{\mathrm{B}} \cdot \vec{\nabla} \hat{K},
$$

where $g$ is the metric determinant of Eq. (2.43), here specialized to the choice $\Psi=\psi$. But Eq. (2.61) implies

$$
\tilde{K}=-(c / e)(d \bar{p} / d \psi+e \bar{n} d \bar{\Phi} / d \psi) g^{1 / 2},
$$

which, according to Eq. (2.44), is independent of $\zeta$. Hence, $\overrightarrow{\mathrm{B}} \cdot \nabla \hat{K}=0$, and the parallel flow is

$$
\left(n u_{\|}\right)_{1}=\hat{n} \cdot(n \overrightarrow{\mathrm{u}})_{1}=-(m \Omega)^{-1} I(d \bar{p} / d \psi+e \bar{n} d \bar{\Phi} / d \psi)+\hat{K}(\psi) B,
$$

where

$$
I(\psi, \Theta, \zeta) \equiv g^{1 / 2} \vec{\nabla} \psi \times \vec{\nabla} \Theta \cdot \overrightarrow{\mathrm{B}} .
$$


A similar argument yields, for the parallel heat flow,

$$
q_{\| 1}=-(5 / 2)(m \Omega)^{-1} I \bar{p} d T / d \psi+\hat{L}(\psi) B .
$$

While the functions $\hat{K}(\psi)$ and $\hat{L}(\psi)$ remain to be determined, Eqs. (2.66) and (2.68) are useful because all the $(\Theta, \zeta)$ dependence is given explicitly in terms of the field geometry.

Equation (2.66) also provides the first-order current

$$
\overrightarrow{\mathrm{J}}=K(\psi) \overrightarrow{\mathrm{B}}-c(d P / d \psi) g^{1 / 2} \vec{\nabla} \psi \times \vec{\nabla} \Theta
$$

or

$$
J_{\|}=K(\psi) B-c(I / B) d P / d \psi,
$$

where

$$
K=\sum_{a} e_{a} \hat{K}_{a}
$$

is a function of $\psi$ only. Note that Eq. (2.69) can also be derived from Eq. (2.40) and the quasi-neutrality condition

$$
\vec{\nabla} \cdot \vec{J}=0 \text {. }
$$

\section{First-order stress}

Equation (2.14) can be considered as the trace of the more general moment

$$
\int d^{3} \overrightarrow{\mathrm{v}} m \overrightarrow{\mathrm{v}} \overrightarrow{\mathrm{v}}[\mathrm{Eq} \cdot(1.1)]
$$

Without writing Eq. (2.73) any more explicitly, we note that it can be reduced to the form [see, for example, Chew, Goldberger and Low (1956)]

$$
\overrightarrow{\mathrm{B}} \times \mathrm{P}+\mathrm{P} \times \overrightarrow{\mathrm{B}}=O\left(\delta^{2}\right) .
$$

Hence, in a coordinate system with one axis along $\vec{B}$, the first-order stress must be diagonal.

$$
\mathrm{P}=\hat{n} \hat{n} \mathrm{P}_{\|}+(I-\hat{n} \hat{n}) \mathrm{P}_{\perp}+O\left(\delta^{2}\right),
$$

where $P_{\|}$and $P_{\perp}$ are, respectively, the parallel and perpendicular stresses. Equation (2.29) requires the stress anisotropy to be at most first order in $\delta$.

This stress tensor describes a plasma in which the various directions of velocity in the plane perpendicular to $\overrightarrow{\mathrm{B}}$ appear uniformly populated, when viewed on time scales long compared to the gyroperiod.

A similar argument yields, for the tensor $R$

$$
\mathbf{R}=\hat{n} \hat{n} \mathbf{R}_{\|}+(1-\hat{n} \hat{n}) \mathbf{R}_{\perp} .
$$

\section{Second-order fluxes}

As suggested by Eqs. (2.20) and (2.63), motion of particles and energy across flux surfaces appears as a second-order process. While closed form expressions for the radial particle and energy fluxes can be obtained only by solving the kinetic equation, useful information is nonetheless available at the fluid level.

Consider first the particle flux. Using Eqs. (2.37) and (2.61), we may write

$$
n \overrightarrow{\mathrm{u}}_{\perp}=n \overrightarrow{\mathrm{u}}_{\perp 1}+n \overrightarrow{\mathrm{u}}_{c}+n \overrightarrow{\mathrm{u}}_{\mathrm{NC}}+O\left(\delta^{3}\right),
$$

where

$$
n \overrightarrow{\mathrm{u}}_{c} \equiv-(m \Omega)^{-1} \hat{n} \times \overrightarrow{\mathrm{F}}
$$

is called the classical flux, and (Wimmel, 1970)

$$
n \overrightarrow{\mathrm{u}}_{\mathrm{NC}} \equiv(m \Omega)^{-1} \hat{n} \times[\vec{\nabla} \cdot(\mathrm{P}-\mathrm{I} \bar{p})-e(n \overrightarrow{\mathrm{E}}+\vec{n} \vec{\nabla} \Phi)]
$$

is called the neoclassical flux. (This terminology, which is roughly conventional, assumes that both fluxes are finally evaluated under the assumption of ordinary Coulomb collisions, as discussed in Sec. I.)

\section{Classical diffusion}

To interpret the flux $n \overrightarrow{\mathbf{u}}_{c}$, we may observe from Eq. (2.61) that the gyration induced diamagnetic drifts for oppositely charged species are in opposite directions. The resulting counter-streaming between ions and electrons is accompanied by collisional friction, $F_{\perp e} \simeq \nu_{e i} m_{e} n_{e}\left(u_{e 1 \perp}-u_{i 1 \perp}\right)$ where $\nu_{e i}$ is the Coulomb collision frequency for electron-ion momentum exchange. (An additional contribution to $\overrightarrow{\mathrm{F}}_{\perp}$, coming from the temperature gradient, is irrelevant to the present, qualitative, argument.) Equation (2.77) then yields a radial flux $n \overrightarrow{\mathrm{u}}_{c} \cong-(\nu / \Omega)(m \Omega)^{-1} \vec{\nabla} \bar{P}$, and correspondingly a classical diffusion coefficient (Rosenbluth and Kaufman, 1958)

$$
D_{c} \simeq 2 \nu_{e i}\left(T_{e} / m_{e}\right) \Omega_{e}^{-2}=\nu_{e i} \rho_{e}^{2},
$$

where $\rho_{e}$ is the electron gyroradius defined by Eq. (2.18).

Equation (2.79) (which happens to be exact when $T_{i}=T_{e}$ ) has been derived for the case of electron diffusion, but in fact in the case of a single ion species, it holds for the ions as well. This is because collisional momentum conservation, Eq. (2.11), guarantees ambipolarity of the classical flux

$$
\sum_{a} e_{a} n_{a} \overrightarrow{\mathrm{u}}_{a c}=0 \text {. }
$$

Notice that Eq. (2.61), and therefore Eq. (2.79), require only

$$
\nu \ll \Omega,
$$

i.e., that the lowest-order gyromotion be unperturbed by collisions. As shown in Sec. III, such nonrandom particle motion drives a non-Maxwellian perturbation on the distribution function, $f-f_{M}=O(\delta)$, and this perturbation in turn drives $\overrightarrow{\mathrm{F}}_{\perp}$.

\section{Neoclassical transport}

In our definition the neoclassical flux has two sources, $\mathrm{P}-\mid \bar{p}$ and $n \overrightarrow{\mathrm{E}}+\bar{n} \vec{\nabla} \bar{\Phi}$. The latter pertains mainly to motion of the magnetic surfaces, and is discussed in Sec. II.E below. Here we consider the typically more important source, $P-I \bar{p}$. It's form and origin depend upon the size of the collision frequency: always assuming $\nu \ll \Omega$, we must also specify the relative magnitudes of $\nu$ and $\omega$. In the collisional regime

$$
\nu \gg \omega \text {, }
$$

the stress anisotropy is kept small by collisional randomization,

$$
\mathrm{P}_{\mathrm{li}}-\mathrm{P}_{\perp}=O(\delta \omega / \nu),
$$

but the mean free path is short enough to allow pressure variation 


$$
p-\bar{p}=O(\delta \nu / \omega)
$$

within the magnetic surface; indeed, in the nonuniform magnetic field of a toroidal confinement system, such variation is required by the parallel equilibrium flows of Eqs. (2.66)-(2.68) (Pfirsch and Schliuter, 1962). The resulting diamagnetic drift, $(m \Omega)^{-1} \hat{n} \times \nabla(p-\bar{p})$, is radially outward, and contributes to $n \overrightarrow{\mathrm{u}}_{\mathrm{NC}}$ a particle flux, often called the Pfirsch-Schluiter flux, which exceeds classical diffusion by a factor of roughly $q^{2}$.

An alternate explanation for Pfirsch-Schlüter transport, in terms of $\nabla B$-drifts superimposed on the stochastic motion of collision-dominated guiding centers, is discussed in Sec. III, and the complete collisional transport problem for a torus is considered in Sec. IV.

In collisionless regimes $(\nu \ll \omega)$, the particles move freely along field lines to maintain $p \simeq \bar{p}$, but an even larger enhancement of diffusion results from the stress anisotropy. The underlying mechanism which supports $\mathrm{P}_{\|}-\mathrm{P}_{\perp}=O(\delta)$ is again the $\nabla B-$ drift, which in this case perturbs free particle motion, as discussed in Sec. III. The kinetic theory appropriate to collisionless regimes is presented in Sec. V.

Notice that in both collision frequency regimes $n \overrightarrow{\mathrm{u}}_{\mathrm{NC}}$ can be related to magnetic field inhomogeneity. Thus in a uniform system (not a confinement system!), it may be assumed that

$$
\mathrm{P}-I \bar{p}=n \overrightarrow{\mathrm{E}}+\bar{n} \vec{\nabla} \bar{\Phi}=0,
$$

and only classical transport need by present. An obvious inference would seem to be that neoclassical transport vanishes in the infinite aspect ratio limit, in which a torus becomes a straight cylinder. However, this turns out to be correct only if the safety factor $q$ also vanishes in the limit. Thus, even for very large aspect ratio, neoclassical effects remain strong, so long as Eq. (2.51) holds.

Similar comments apply to the energy flux. Thus Eq. (2.38) may be written as

$$
\overrightarrow{\mathrm{Q}}_{\perp}=\overrightarrow{\mathrm{Q}}_{\perp 1}+\overrightarrow{\mathrm{Q}}_{c}+\overrightarrow{\mathrm{Q}}_{\mathrm{NC}}+O\left(\delta^{3}\right),
$$

where $\vec{Q}_{\perp 1}=\vec{q}_{\perp 1}+(5 / 2) \vec{p}_{\perp 1}$ [cf. Eqs. (2.30) and (2.62)], and

$$
\begin{aligned}
& \overrightarrow{\mathrm{Q}}_{c} \equiv-\Omega^{-1} \hat{n} \times \overrightarrow{\mathrm{G}} \\
& \overrightarrow{\mathrm{Q}}_{\mathrm{NC}} \equiv \Omega^{-1} \hat{n} \times\left\{\vec{\nabla} \cdot\left(\mathrm{R}-\frac{5}{2} \frac{\bar{p}}{m} \bar{T} 1\right)-\frac{5}{2} \frac{e}{m}(p \overrightarrow{\mathrm{E}}+\bar{p} \vec{\nabla} \bar{\Phi})\right. \\
&\left.+\frac{e}{m} \vec{\nabla} \bar{\Phi} \cdot\left[\mathrm{P}-\mid \bar{p}-\frac{3}{2} I(p-\bar{p})\right]\right\},
\end{aligned}
$$

are the classical and neoclassical contributions, respectively.

\section{E. Axisymmetric systems}

The transport theory for a rigorously axisymmetric torus is relatively complete, not only because of its comparative theoretical simplicity, but also because this is the more relevant case: asymmetry is purposefully minimized in the design of most present confinement systems. Axisymmetry provides essentially two sources of simplification in our previous formulae. The first is related to the axisymmetric version of Ampere's law, and pertains mainly to the plasma equilibrium. The sec- ond is related to conservation of angular momentum, and pertains to the form of the second order fluxes.

\section{Ampere's law}

We may assume that $\psi$ and $\Theta$ vary orthogonally to the ignorable coordinate $\zeta$, whence

$$
R^{2} \vec{\nabla} \zeta=g^{1 / 2} \vec{\nabla} \psi \times \vec{\nabla} \Theta
$$

where

$$
R=|\nabla \zeta|^{-1}
$$

denotes the major radius, i.e., the distance from the axis of symmetry. From Eq. (2.67) we see that $R \vec{\nabla} \zeta \cdot \overrightarrow{\mathrm{B}}$ $=I / R$, so that Eq. (2.48) becomes

$$
\overrightarrow{\mathrm{B}}=I \vec{\nabla} \zeta+\vec{\nabla} \zeta \times \vec{\nabla} \psi
$$

Now consider Ampere's law, Eq. (2.42). The radial component requires

$$
\partial I / \partial \Theta=0,
$$

i.e., that $I$ is a function of $\psi$ only; the resulting simplification of Eqs. (2.66)-(2.68) and (2.87) is significant. The poloidal component relates $I(\psi)$ to the function $K(\psi)$ of Eq. (2.71)

$$
d I / d \psi=-(4 \pi / c) K(\psi) \text {. }
$$

Finally, the toroidal component of Ampere's law can be reduced to the form (Grad and Rubin, 1958; Laing, et al., 1959; Shafranov, 1958)

$$
R^{2} \vec{\nabla} \cdot\left(R^{-2} \vec{\nabla} \psi\right)=-I d I / d \psi-4 \pi R^{2} d P / d \psi .
$$

This relation is frequently used to determine the equilibrium flux surface geometry, $\psi(\overrightarrow{\mathrm{x}})$.

\section{Second-order fluxes}

In the flux surface averages of the even moment equations, the particle and energy fluxes enter only through their averaged, radial components, $\langle n \overrightarrow{\mathrm{u}} \cdot \vec{\nabla} \psi\rangle$ and $\langle\overrightarrow{\mathrm{Q}} \cdot \vec{\nabla} \psi\rangle$, respectively [recall Eq. (2.57)]. We could compute these averages for the axisymmetric case by specializing our previous formulae, Eqs. (2.76) and (2.83), but it is simpler to proceed directly from the moment equations, Eqs. (2.15) and (2.16) (Hazeltine, 1975).

Consider first the particle flux. Conservation of angular momentum suggests that we multiply Eq. (2.15) by $(c / e) R^{2} \vec{\nabla} \zeta$ and perform the average; observe that

$$
\left\langle R^{2} \vec{\nabla} \zeta \cdot \vec{\nabla} \cdot \mathrm{P}\right\rangle=\left\langle\vec{\nabla} \cdot\left(R^{2} \vec{\nabla} \zeta \cdot \mathrm{P}\right)\right\rangle=\frac{d}{d V}\left\langle\mathrm{P}_{\zeta}^{V}\right\rangle,
$$

where

$$
\mathrm{P}_{\zeta}^{V} \equiv R^{2} \vec{\nabla} \zeta \cdot \mathrm{P} \cdot \vec{\nabla} V=O\left(\delta^{2}\right),
$$

and we have used Eqs. (2.57) and (2.74). The remaining terms in Eq. (2.15) yield simply

$$
\langle n \overrightarrow{\mathrm{u}} \cdot \vec{\nabla} \psi\rangle=-(c / e)\left\langle R^{2} \vec{\nabla} \zeta \cdot(\overrightarrow{\mathrm{F}}+e n \overrightarrow{\mathrm{E}})\right\rangle+O\left(\delta^{3}\right) .
$$

This expression contains both classical and neoclassical contributions. These may be distinguished by noting from Eq. (2.77) that

$$
\left\langle n \overrightarrow{\mathrm{u}}_{c} \cdot \vec{\nabla} \psi\right\rangle=-(c / e)\left\langle R^{2} \vec{\nabla} \zeta \cdot \overrightarrow{\mathrm{F}}_{\perp}\right\rangle,
$$

and therefore that 


$$
\left\langle n \overrightarrow{\mathrm{u}}_{\mathrm{NC}} \cdot \vec{\nabla} \psi\right\rangle=-(c / e)\left\langle R^{2} \vec{\nabla} \zeta \cdot\left(\overrightarrow{\mathrm{F}}_{\| \mid}+e n \overrightarrow{\mathrm{E}}\right)\right\rangle .
$$

Thus (aside from the $E_{T}=R \vec{\nabla} \zeta \cdot \overrightarrow{\mathrm{E}}$ terms, which shall be interpreted later), the classical and neoclassical fluxes are closely analogous: the first is driven by perpendicular friction just as the second is driven by parallel friction. The more fundamental analogy, between the gyromotion giving classical transport and the guiding center motion giving neoclassical transport, is considered in Sec. III.

Notice that the parallel component of Eq. (2.15), with Eq. (2.74), requires

$$
F_{\|}+e n E_{\|} \simeq \hat{n} \cdot \vec{\nabla} \mathrm{P}_{\|}-\left(\mathrm{P}_{\|}-\mathrm{P}_{\perp}\right) \hat{n} \cdot \vec{\nabla} B / B \text {. }
$$

In this way, the stress anisotropy and poloidal pressure variation, which were previously emphasized, enter into $\mathrm{Eq}$. (2.95). In fact, using Eq. (2.96) and the relations

$$
\begin{aligned}
& \hat{n} \times \vec{\nabla} \cdot \mathrm{P}=\hat{n} \times \vec{\nabla} \mathrm{P}_{\perp}+\left(\mathrm{P}_{\|}-\mathrm{P}_{\perp}\right) \hat{n} \times(\hat{n} \cdot \vec{\nabla}) \hat{n}, \\
& \vec{\nabla} \psi \cdot \hat{n} \times \overrightarrow{\mathrm{E}}=-I E_{\|}+B R^{2} \vec{\nabla} \zeta \cdot \overrightarrow{\mathrm{E}},
\end{aligned}
$$

the equivalence of Eqs. (2.78) and (2.95) can straightforwardly be demonstrated (Bernstein, 1974). We omit the details.

Note also that in an axisymmetric system, collisional momentum conservation guarantees ambipolarity of the total second-order particle flux (Kovrizhnikh, 1969; Rutherford, 1970)

$$
\sum_{a} e_{a}\left\langle n_{a} \overrightarrow{\mathrm{u}}_{a} \cdot \vec{\nabla} \psi\right\rangle=0 ;
$$

compare Eq. (2.80).

Finally, we consider the energy flux. The same manipulations used to derive Eq. (2.93) yield, when applied to Eq. (2.16), the expression

$$
\langle\overrightarrow{\mathrm{Q}} \cdot \vec{\nabla} \psi\rangle=-(c / e)\left\langle R^{2} \vec{\nabla} \zeta \cdot(m \overrightarrow{\mathrm{G}}+5 e p \overrightarrow{\mathrm{E}} / 2)\right\rangle+O\left(\delta^{3}\right),
$$

where $\vec{G}$ is given by Eq. (2.10). The classical and neoclassical contributions are, respectively,

$$
\begin{aligned}
& \left\langle\overrightarrow{\mathrm{Q}}_{c} \cdot \vec{\nabla} \psi\right\rangle=-(c / e)\left\langle R^{2} \vec{\nabla} \zeta \cdot m \overrightarrow{\mathrm{G}}_{\perp}\right\rangle, \\
& \left\langle\overrightarrow{\mathrm{Q}}_{\mathrm{NC}} \cdot \vec{\nabla} \psi\right\rangle=-(c / e)\left\langle R^{2} \vec{\nabla} \zeta \cdot\left(m \overrightarrow{\mathrm{G}}_{\|}+5 p \overrightarrow{\mathrm{E}} / 2\right)\right\rangle .
\end{aligned}
$$

\section{Faraday's law}

Faraday's law

$$
\vec{\nabla} \times \overrightarrow{\mathrm{E}}=-c^{-1} \partial \overrightarrow{\mathrm{B}} / \partial t,
$$

is important primarily for a tokamak plasma, in which the poloidal magnetic field, being supported by plasma current, can change on the diffusion time scale. Hence the consequences of Eq. (2.103) have been studied extensively only in axisymmetric geometry.

The poloidal and toroidal components of Faraday's law are needed to describe magnetic field diffusion, and therefore to obtain a closed set of transport equations; this matter is considered in Sec. VII. Here we consider only some simple consequences of the radial component. From $\vec{\nabla} \psi \cdot \vec{\nabla} \times \overrightarrow{\mathrm{E}}=\overrightarrow{\mathrm{B}} \cdot \vec{\nabla} R E_{T}$ and $\vec{\nabla} \psi \cdot \partial \overrightarrow{\mathrm{B}} / \partial t=-\overrightarrow{\mathrm{B}} \cdot \vec{\nabla} \partial \psi / \partial t$ we have $\overrightarrow{\mathrm{B}} \cdot \vec{\nabla}\left(c R E_{T}-\partial \psi / \partial t\right)=0$. We may therefore take

$$
\partial \psi / \partial t=c R E_{T},
$$

or

$$
\psi=-R A_{T},
$$

where $A_{T} \equiv R \vec{\nabla} \zeta \cdot \overrightarrow{\mathrm{A}}$ is the toroidal component of the vector potential. Note that with this choice $\psi$ vanishes not on the magnetic axis, as Eq. (2.45) would suggest, but rather on the symmetry axis. In fact $R A_{T}$ measures the poloidal flux outside a given flux surface. This circumstance has no effect on our previous analysis, which involved only the spatial dependence of $\psi$.

The velocity $\overrightarrow{\mathrm{u}}_{s}$ of a flux surface may be defined by

$$
\partial \psi / \partial t+\overrightarrow{\mathrm{u}}_{s} \cdot \vec{\nabla} \psi=0,
$$

since only the radial flux surface motion is physically significant. The relation

$$
\overrightarrow{\mathrm{u}}_{s} \cdot \vec{\nabla} \psi=-c R E_{T}
$$

provides a useful interpretation of the $E_{T}$ term in the radial particle flux. Thus Eq. (2.93) may be written as

$$
\left\langle n\left(\overrightarrow{\mathrm{u}}-\overrightarrow{\mathrm{u}}_{\mathrm{s}}\right) \cdot \vec{\nabla} \psi\right\rangle=-(c / e)\left\langle R F_{\boldsymbol{T}}\right\rangle+O\left(\delta^{3}\right),
$$

and we see that radial diffusion, relative to a poloidal flux surface, is proportional to the friction force.

It is instructive to consider a third version of Eq. (2.93). If we use Eq. (2.98) to express the toroidal electric field $R \vec{\nabla} \zeta \cdot \overrightarrow{\mathrm{E}}=-c^{-1} R \vec{\nabla} \zeta \cdot \partial \overrightarrow{\mathrm{A}} / \partial t$ in terms of its components perpendicular and parallel to $\vec{B}$, we obtain

$$
\begin{aligned}
\langle n \overrightarrow{\mathrm{u}} \cdot \vec{\nabla} \psi\rangle= & \left\langle\vec{\nabla} \psi \cdot n B^{-1} \hat{n} \times \partial \overrightarrow{\mathrm{A}} / \partial t\right\rangle \\
& +I\left\langle n B^{-1} \hat{n} \cdot \partial \overrightarrow{\mathrm{A}} / \partial t\right\rangle-(c / e)\left\langle R F_{T}\right\rangle .
\end{aligned}
$$

In this form the dissipationless flux $\left(F_{T}=0\right)$ is most easily understood. Note that a lowest-order equilibrium without friction can exist only if $\hat{n} \cdot \partial \overrightarrow{\mathrm{A}} / \partial t$ also vanishes, since otherwise charged particles would be accelerated indefinitely. Hence, in the limit of no dissipation, only the first term of Eq. (2.109), giving the inductive $\overrightarrow{\mathrm{E}} \times \overrightarrow{\mathrm{B}}$ drift, survives, and Eq. (2.109) becomes the statement of "frozen" field lines, as in ideal magnetohydrodynamics.

With dissipation, on the other hand, Eq. (2.98) shows that the velocity $\overrightarrow{\mathrm{u}}_{s} \cdot \vec{\nabla} \psi /|\nabla \psi| \simeq-c E_{T} / B_{p}$ is typically much faster than an ordinary $\overrightarrow{\mathrm{E}} \times \overrightarrow{\mathrm{B}}$ drift (Klima, 1965).

Similar remarks pertain to the energy flux of Eq. (2.100), which may be written in the form

$$
\left\langle\left(\overrightarrow{\mathrm{Q}}-(5 / 2) p \overrightarrow{\mathrm{u}}_{s}\right) \cdot \vec{\nabla} \psi\right\rangle=-(c / e)\left\langle R^{2} \vec{\nabla} \zeta \cdot m \overrightarrow{\mathrm{G}}\right\rangle+O\left(\delta^{3}\right) .
$$

\section{F. Entropy and heat production}

The lowest-order entropy density is defined, at the fluid level, by

$$
s_{a}=\ln \left(T_{a}^{3 / 2} / n_{a}\right)
$$

(we suppress the over-bars: $T_{a}=\bar{T}_{a}$, etc.). Since the entropy flux is $n_{a} s_{a} \overrightarrow{\mathrm{u}}_{a}+\overrightarrow{\mathrm{q}}_{a} / T_{a}$, the rate of collisional entropy production is given by

$$
\Theta_{a} \equiv(\partial / \partial t) n_{a} s_{a}+\vec{\nabla} \cdot\left(n_{a} s_{a} \overrightarrow{\mathrm{u}}_{a}+\overrightarrow{\mathrm{q}}_{a} / T_{a}\right) .
$$

It can be said that the object of transport theory is a sufficiently explicit evaluation of $\Theta_{a}$. A more useful, related quantity is the rate of heat production

$$
\mathfrak{H}=\sum_{a} T_{a} \Theta_{a} .
$$


After substituting the conservation laws, Eqs. (2.13) and (2.14), into Eq. (2.111), and using Eq. (2.30), we obtain

$$
\begin{aligned}
\Theta_{a}= & -\overrightarrow{\mathrm{u}}_{a} \cdot \vec{\nabla} p_{a} / T_{a}-\overrightarrow{\mathrm{q}}_{a} \cdot \vec{\nabla} T_{a} / T_{a}^{2} \\
& +Q_{a} / T_{a}+\overrightarrow{\mathrm{u}}_{a} \cdot\left(\overrightarrow{\mathrm{F}}_{a}+e_{a} n_{a} \overrightarrow{\mathrm{E}}\right) / T_{a} .
\end{aligned}
$$

Therefore, using Eqs. (2.11) and (2.12), we have

$$
\mathfrak{H C}=-\sum_{a}\left\{\overrightarrow{\mathrm{u}}_{a} \cdot \vec{\nabla} p_{a}+\overrightarrow{\mathrm{q}}_{a} \cdot \vec{\nabla} T_{a} / T_{a}\right\}+\overrightarrow{\mathrm{J}} \cdot \overrightarrow{\mathrm{E}} .
$$

In this form, the irreversible nature of $\mathcal{H}$ is apparent: Eq. (2.113) has the characteristic form of a sum of products of forces $\left(\vec{\nabla} p_{a}, \vec{\nabla} T_{a} / T_{a}, \overrightarrow{\mathrm{E}}\right)$ and fluxes $\left(\overrightarrow{\mathrm{u}}_{a}, \overrightarrow{\mathrm{q}}_{a}, \vec{J}\right)$.

Next, we specialize to an axisymmetric system, and consider the flux-surface average of $\mathfrak{H}$. After making use of Eqs. (2.69), (2.86), (2.89), and (2.93), we obtain

$$
\begin{aligned}
\langle\mathcal{H C}\rangle= & -\sum_{a}\left\{\left\langle\overrightarrow{\mathrm{q}}_{a} \cdot \vec{\nabla} \psi\right\rangle d \ln T_{a} / d \psi+\left(c / e_{a}\right)\left\langle R F_{T a}\right\rangle n_{a}^{-1} d p_{a} / d \psi\right\} \\
& -(c / 4 \pi)\left\langle E_{\|} B\right\rangle d I / d \psi .
\end{aligned}
$$

Here, the first term requires no interpretation. The second term involves the radial particle flux, relative to the moving poloidal flux surface, as shown by Eq. (2.108). To understand the last term of Eq. (2.114), note that the Poynting flux relative to the poloidal flux surface is given by $(c / 4 \pi)\left[\overrightarrow{\mathrm{E}}+c^{-1} \overrightarrow{\mathrm{u}}_{s} \times \overrightarrow{\mathrm{B}}\right] \times \overrightarrow{\mathrm{B}}$, where $\overrightarrow{\mathrm{u}}_{s}$ is the flux surface velocity of Eq. (2.106). Averaging the radial component over a flux surface, and using Eq. (2.107), we obtain

$$
(c / 4 \pi)\left\langle\left[\left(\overrightarrow{\mathrm{E}}+c^{-1} \overrightarrow{\mathrm{u}}_{s} \times \overrightarrow{\mathrm{B}}\right) \times \overrightarrow{\mathrm{B}}\right] \cdot \vec{\nabla} \psi\right\rangle=(c I / 4 \pi)\left\langle E_{\|} B\right\rangle .
$$

Hence the last term of Eq. (2.114) is simply the product of the relative Poynting flux and its corresponding "force," $d \ln I / d \psi$.

Thus, the electric field enters our final results only through $\left\langle E_{\|} B\right\rangle$ and, implicitly, through $\overrightarrow{\mathrm{u}}_{s} \cdot \vec{\nabla} \psi=-c R E_{T}$. It shall be seen in later sections that this circumstance is characteristic of an axisymmetric confinement system (even when slightly different choices of fluxes and forces are found more convenient). Note in particular that the electrostatic potential does not appear, because of Eq. (2.53).

\section{GUIDING CENTER EQUATIONS}

\section{A. Basic formalism}

For small gyroradius, the particle velocity, $\vec{v}=\vec{v}_{\|}+\vec{v}_{\perp}$, is most conveniently parametrized in terms of the magnetic moment

$$
\mu=v_{\perp}^{2} / 2 B,
$$

the energy

$$
\dot{\epsilon}=v^{2} / 2+e \Phi / m,
$$

and the gyrophase angle,

$$
\zeta=-\tan ^{-1}\left(\overrightarrow{\mathrm{v}} \cdot \hat{e}_{2} / \overrightarrow{\mathrm{v}} \cdot \hat{e}_{3}\right),
$$

where the unit vectors $\hat{e}_{2}$ and $\hat{e}_{3}$ are chosen to make $\left(\hat{n}, \hat{e}_{2}, \hat{e}_{3}\right)$ a right-handed orthogonal triplet at each point. Thus

$$
\overrightarrow{\mathrm{v}}_{\perp}=(2 \mu B)^{1 / 2}\left[\hat{e}_{2} \cos \zeta-\hat{e}_{3} \sin \zeta\right] .
$$

Note that in Eq. (3.1) we measure $\mu$ in a fixed reference frame, rather than in one moving with the $\vec{E} \times \vec{B}$ drift. This reflects our choice of the drift ordering, Eq. (2.21), rather than the magnetohydrodynamical ordering, $c E / B$ $\sim v_{\text {th }}$. Note also that the drift ordering, with Faraday's law, requires only $d \ln B / d t=O(\delta \omega)$; but the stronger assumption

$$
d \ln B / d t=O\left(\delta^{2} \omega\right)
$$

which is implied by Eq. (2.20), is more relevant to transport theory, and is adopted here.

The equation of motion

$$
\frac{d \overrightarrow{\mathrm{v}}}{d t}=\frac{e}{m}\left[\overrightarrow{\mathrm{E}}(\overrightarrow{\mathrm{x}}, t)+c^{-1} \overrightarrow{\mathrm{v}} \times \overrightarrow{\mathrm{B}}(\overrightarrow{\mathrm{x}}, t)\right]
$$

can be written in terms of $\mu, \epsilon$, and $\zeta$, with the result

$$
\begin{aligned}
& \frac{d \mu}{d t}=\frac{-\mu}{B} \frac{d B}{d t}-\frac{\overrightarrow{\mathrm{v}}_{\|}}{B} \cdot \frac{d \hat{n}}{d t}+\frac{e}{m B} \overrightarrow{\mathrm{v}}_{\perp} \cdot \overrightarrow{\mathrm{E}}, \\
& \frac{d \epsilon}{d t}=\frac{e}{m}\left(\frac{d \Phi}{d t}+\overrightarrow{\mathrm{v}} \cdot \overrightarrow{\mathrm{E}}\right), \\
& \frac{d \zeta}{d t}=\Omega+\hat{e}_{3} \cdot \frac{d \hat{e}_{2}}{d t}+\frac{\hat{n} \times \overrightarrow{\mathrm{v}}_{\perp}}{v_{\perp}^{2}} \cdot\left(v_{\|} \frac{d \hat{n}}{d t}-\frac{e}{m} \overrightarrow{\mathrm{E}}\right),
\end{aligned}
$$

where, on the right-hand sides, $d / d t \equiv \partial / \partial t+\overrightarrow{\mathrm{v}} \cdot \vec{\nabla}$.

Equations describing the motion of the guiding center (g.c.) are obtained by averaging Eqs. (3.6)-(3.9) over a gyroperiod. ${ }^{2}$ We first define the instantaneous g.c. position $\vec{R}$, of a particle at the point $\vec{x}$, by

$$
\overrightarrow{\mathrm{R}} \equiv \overrightarrow{\mathrm{x}}-\vec{\rho} \text {, }
$$

where $\vec{\rho}$ is the gyroradius vector

$$
\vec{\rho}=\hat{n} \times \vec{v}_{\perp} / \Omega \text {. }
$$

Hence

$$
\frac{d \overrightarrow{\mathrm{R}}}{d t}=\overrightarrow{\mathrm{v}}-\frac{d \vec{\rho}}{d t}
$$

Next, the gyrophase average of any function $f$ is defined by

$$
\bar{f} \equiv \oint(d \zeta / 2 \pi) f,
$$

the integral being performed at constant $\mu, \epsilon, \vec{x}, t$. The g.c. velocity is given by the average of Eq. (3.12),

$$
\overrightarrow{\mathrm{v}}_{\mathrm{gc}} \equiv \overline{d \overrightarrow{\mathrm{R}} / d t}=\overrightarrow{\mathrm{v}}_{\|}-\overline{d \vec{\rho} / d t} \text {. }
$$

After substituting Eq. (3.11) into Eq. (3.14), and using Eqs.(3.7)-(3.9), we find

$$
\overrightarrow{\mathrm{v}}_{\mathrm{gc}}=\hat{n}\left(v_{\|}+\hat{v}_{\|}\right)+\overrightarrow{\mathrm{v}}_{D},
$$

where

$$
\hat{v}_{\|}=\left(v_{\perp}^{2} / 2 \Omega\right) \hat{n} \cdot \vec{\nabla} \times \hat{n},
$$

and

$$
\overrightarrow{\mathrm{v}}_{D}=c \overrightarrow{\mathrm{E}} \times \hat{n} / B+\hat{n} \times\left(\mu \vec{\nabla} B+v_{\|}^{2} \hat{n} \cdot \vec{\nabla} \hat{n}+v_{\|} \partial \hat{n} / \partial t\right) / \Omega
$$

${ }^{2}$ A lucid treatment of the averaging is presented by Baños (1967) who however, uses the magnetohydrodynamical ordering. More extensive treatments of the g.c. theory may be found in, for example, Northrop (1963) Hastie et al. (1967), and Morosov and Solov'ev (1966); the last of these is most relevant to transport theory. 
are, respectively, the parallel and perpendicular g.c. drifts. The first term of Eq. (3.17) is the familiar $\overrightarrow{\mathrm{E}} \times \overrightarrow{\mathrm{B}}$ drift, the second term is called the gradient- $B \mathrm{drift}$, and the third term is called the curvature drift. The third and fourth terms together comprise the "acceleration drift," in the terminology of Northrop (1963). The various perpendicular drifts may be understood in terms of distortions of the gyro-orbit; see, for example, Rosenbluth and Longmire (1957).

Equations (3.15)-(3.17) are exact. The small $\delta$ assumption enters in the statement that when viewed on time scales long compared to the gyroperiod, the exact particle trajectory may be approximated by $\overrightarrow{\mathrm{v}}_{\mathrm{gc}}$.

Thus the lowest-order g.c. motion consists of a streaming along the magnetic field lines.

$$
\overrightarrow{\mathrm{v}}_{\mathrm{gc}}=\hat{n} v_{\|}[1+O(\delta)] \text {, }
$$

where

$$
v_{\|}= \pm[2(\epsilon-\mu B-e \Phi / m)]^{1 / 2} .
$$

In a nonuniform field, even this motion displays interesting features, because Eqs. (3.7) and (3.8) imply

$$
\begin{aligned}
& \overline{d \mu / d t}=-(\mu / B) \partial B / \partial t, \\
& \overline{d \epsilon / d t}=(e / m)\left[\partial \Phi / \partial t-c^{-1} v_{\|} \hat{n} \cdot \partial \overrightarrow{\mathrm{A}} / \partial t\right],
\end{aligned}
$$

i.e., that the averaged changes in $\mu$ and $\epsilon$ are relatively small. Hence a particle with sufficiently large perpendicular energy will be "trapped"-confined to regions of the magnetic surface in which $\mu B(\overrightarrow{\mathrm{x}})<\epsilon-e \Phi(\overrightarrow{\mathrm{x}}) / m$.

The first-order drift terms can be conveniently simplified, for the important case of small $\beta=8 \pi P / B^{2}$. Assuming $\beta=O(\delta)$ we find from Ampere's law and Eq. (2.40) that

$$
\hat{n} \cdot \vec{\nabla} \hat{n}-\vec{\nabla}_{\perp} B / B=4 \pi \vec{\nabla} p / B^{2}=O(\beta),
$$

so that the curvature and $\nabla B$ drifts may be combined. Similarly, $\hat{n} \cdot \vec{\nabla} \times \hat{n}=O(\beta)$, assuming $J_{\|} / J_{\perp} \sim 1$. Finally, treating the time derivatives in Eq. (3.17) as second order, and recalling from Eq. (2.31) that the electric field is mainly electrostatic, we find that Eq. (3.17) may be written as

$$
\overrightarrow{\mathrm{v}}_{\mathrm{gc}}=\hat{n} v_{\|}+\overrightarrow{\mathrm{v}}_{d}+O\left(\delta^{2}\right),
$$

where

$$
\overrightarrow{\mathrm{v}}_{d}=-v_{\|} \hat{n} \times \vec{\nabla}\left(v_{\|} / \Omega\right) .
$$

Here the gradient is to be performed at constant $\mu$ and $\epsilon$, using Eq. (3.19). This result was apparently first obtained by Alfven (1950).

\section{B. Drift surfaces}

Following Morosov and Solov'ev (1966), we may introduce the modified vector potential

$$
\overrightarrow{\mathrm{A}}_{*}=\overrightarrow{\mathrm{A}}+v_{\|} \overrightarrow{\mathrm{B}} / \Omega \text {, }
$$

with corresponding field

$$
\overrightarrow{\mathrm{B}}_{*}=\vec{\nabla} \times \overrightarrow{\mathrm{A}} * \text {. }
$$

Then Eq. (3.23) becomes (with the consistent neglect of small $\beta$ corrections)

$$
\overrightarrow{\mathrm{v}}_{\mathrm{gc}}=\left(\overrightarrow{\mathrm{B}}_{*} / B\right) v_{\|},
$$

formally similar to Eq. (3.18). To pursue this analogy, one presumes the existence of a function $\Psi_{*}(\vec{x}, \mu, \epsilon)$ satisfying

$$
\overrightarrow{\mathrm{B}}_{*} \circ \vec{\nabla} \Psi_{*}=0,
$$

analogously to $\overrightarrow{\mathrm{B}} \cdot \vec{\nabla} \Psi=0$. The surfaces corresponding to fixed values of $\mu, \epsilon$, and $\Psi_{*}$ are called drift surfaces; Eq. (3.28) states that the first order g.c. motion is confined to a single drift surface, just as the zeroth-order motion is confined to a single flux surface.

While the family of trajectories $\vec{B} *(\mu, \epsilon)$ are generally well-defined, the existence of drift surfaces can be guaranteed only for confinement geometries possessing appropriate symmetry. Then $\Psi_{*}$ appears as the dynamical invariant, in a g.c. Lagrangian sense, corresponding to the ignorable coordinate (Morosov and Solev'ev, 1966). In an axisymmetric toroidal system, for example, $\Psi_{*}$ is realized as the gyrophase-averaged, canonical angular momentum. We consider this case in further detail.

\section{Axisymmetric systems}

The predominant magnetic field variation in an axisymmetric (or nearly axisymmetric) toroidal system results from

$$
B \simeq B_{T} \propto R^{-1},
$$

where $R$ is the major radius [cf. Eq. $(2.87)$ ].

With regard to the zeroth-order g.c. motion, Eq. $(3.29)$ implies that trapped particles reside mainly in the outer (larger $R$ ) regions of the torus. With regard to the first-order motion, it implies that the drift due to magnetic field inhomogeneity is approximately vertical: positively (negatively) charged particles drift downwards (upwards), as can be seen from Eq. (3.17). These facts determine the character of g.c. orbits in a torus. Notice in particular that when projected onto the $(\Psi, \theta)$ plane, the orbits appear to be closed, as shown in Fig. 2. (Morosov and Solov'ev, 1966; Berk and Galeev, 1967). The net radial drift, after one bounce (trapped particle), or one poloidal circuit around the magnetic axis (untrapped particle), is zero, because in the presence of $\vec{B}_{p} \neq 0$, the g.c.'s lowest-order motion causes it to spend equal times above the magnetic axis (where an electron, say, drifts radially outward) and below the magnetic axis (where an electron drifts inward).

The collisionless orbits remain within a fixed distance of a single flux surface essentially because they are confined to surfaces of constant canonical angular momentum, $R^{2} \vec{\nabla} \zeta \cdot(m \overrightarrow{\mathrm{v}}+e \overrightarrow{\mathrm{A}} / c)$. Recalling Eq. (2.105), we may write this constraint as

$$
\psi-c m v_{T} R / e=\text { const., }
$$

where $v_{T}=R \vec{\nabla} \zeta \cdot \overrightarrow{\mathrm{v}}$ is the toroidal velocity. A gyrophase average provides the useful drift surface label

$$
\Psi_{*}=\psi-I v_{\|} / \Omega+O\left(\delta^{2}\right),
$$

where $I$ is defined by Eq. (2.87), and we have recalled Eq. (3.18).

Now consider the equation $\overrightarrow{\mathrm{v}}_{\mathrm{gc}} \cdot \vec{\nabla} \Psi_{*}=0$, which defines the drift surface. The first order terms come from $v_{\|} \hat{n} \cdot \vec{\nabla} \Psi_{*}$ and $\vec{v}_{D} \cdot \vec{\nabla} \psi$; we find 


$$
\overrightarrow{\mathrm{v}}_{D} \circ \vec{\nabla} \psi=I v_{\|} \hat{n} \circ \vec{\nabla}\left(v_{\|} / \Omega\right)+O\left(\delta^{2}\right) .
$$

The agreement between this expression, which holds for any $\beta$, and the axisymmetric version of Eq. (3.24), which assumes $\beta=O(\delta)$, may seem surprising. But Eq. (3.24) omits only a term proportional to $\hat{n} \times \vec{\nabla} p$, as shown by Eq. (3.22), and axisymmetry implies $\vec{\nabla} \psi \cdot \hat{n} \times \nabla p$ $=-I \hat{n} \cdot \vec{\nabla} p=O(\delta)$, for any $\beta$, as noted in Eq. (2.35).

A more interesting result follows from the exact invariant of Eq. (3.30):

$$
d \psi / d t=(\mathrm{cm} / e) d\left(R v_{T}\right) / d t,
$$

where the derivative is taken along the particle trajectory. Considering only the trapped particles, we integrate Eq. (3.33) between two successive times $t_{1}$ and $t_{2}$ at which $v_{T}$ vanishes, and obtain

$$
\left.\psi\left(t_{2}\right)-\psi\left(t_{1}\right)=\overline{(\partial \psi / \partial t}+\overline{\vec{v} \cdot \vec{\nabla} \psi}\right)\left(t_{2}-t_{1}\right)=0,
$$

where the overbar denotes an orbital average. Equation (2.106) then provides the result

$$
\overline{\overrightarrow{\mathrm{v}} \circ \vec{\nabla} \psi}=\overline{\overrightarrow{\mathrm{u}}_{s} \cdot \vec{\nabla} \psi}=\overline{-c R E_{T}} .
$$

Hence, the trapped particles remain, on the average, tied to the poloidal flux surface, even as this surface moves in response to toroidal electric fields. The resulting $c E_{T} / B_{p}$ inward motion ("trapped particle pinch effect"), which bears significantly on transport in a tokamak, was discovered by Ware (1970) and independently by Galeev (1971).

In Sec. II we pointed out that, without collisions, the induced, parallel electric field must be presumed to vanish [cf. the discussion concerning Eq. (2.109)]. In this case the velocity $\overrightarrow{\mathrm{u}}_{s}$ coincides with an $\overrightarrow{\mathrm{E}} \times \overrightarrow{\mathrm{B}}$ drift in the induced electric field [recall Eq. (2.98)], and Eq. (3.35) appears trivial. It is not trivial because it remains approximately correct in the presence of collisions, when $\overrightarrow{\mathrm{u}}_{s}$ is typically much faster than the $\overrightarrow{\mathrm{E}} \times \overrightarrow{\mathrm{B}}$ drift, provided the collision frequency is sufficiently small (Rutherford, et al., 1970). This surprising circumstance can be thoroughly explained only from the kinetic considerations of Sec. V. However, a partial explanation is possible here. Note first that, when acting on the untrapped particles, the (parallel components of the) accelerating toroidal electric field and decelerating toroidal friction force tend to balance. Untrapped particles therefore experience, roughly, only the relatively slow $\overrightarrow{\mathrm{E}} \times \overrightarrow{\mathrm{B}}$ drift; see Eq. (2.109). For the trapped particles, on the other hand, the parallel electric field, which always acts in the same direction, cannot be balanced by the parallel friction force, which changes direction each time the trapped particle is reflected. The kinetic analysis of Sec. V shows that for these particles the effect of friction, when averaged over the collisionless orbit, is so small that Eq. (3.35) remains roughly valid.

Equation (3.31) may be used to estimate the radial excursion of a g.c. from a given flux surface. Let $\Delta f$ denote the change in $f$ which occurs on the lowest-order orbit. Then $\Delta \Psi_{*}=0$ implies that the poloidal flux change associated with the excursion is $\Delta \psi=I \Delta\left(v_{\|} / \Omega\right)$; the excursion in minor radius, $r$, is $\Delta r=\Delta \psi /|\nabla \psi|$ or

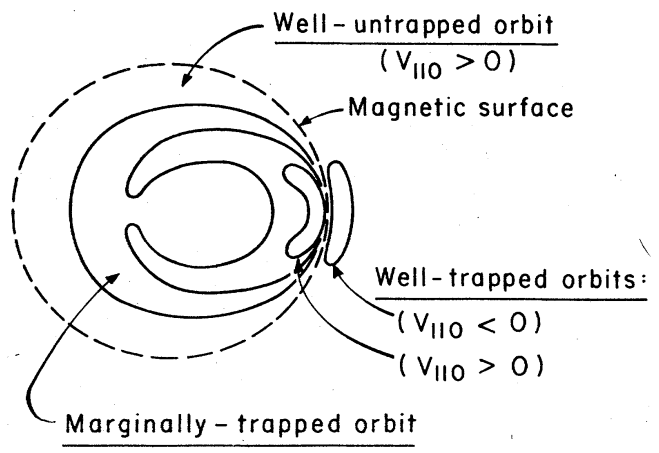

FIG. 2. Trapped and untrapped guiding center orbits, in an axisymmetric system, projected onto a constant- $\zeta$ plane. The dashed line is the projection of the magnetic surface to which each orbit shown is tangent; the parallel velocity at the position of that magnetic surface is labeled $v_{\|_{0}}$. The signs indicated for $v_{\| 0}$ correspond to a positively charged particle, when the toroidal magnetic field and current are in the direction out of the paper. The designations "well-untrapped" and "well-trapped" refer to the cases $\left|v_{110}\right| \gg v_{\perp 0}(\Delta B / B)^{1 / 2}$ and $\left|v_{110}\right| \ll v_{\perp 0}(\Delta B / B)^{1 / 2}$, respectively, while "marginally trapped" refers to the case $\left|v_{10}\right| \lesssim v_{\perp 0}(\Delta B / B)^{1 / 2}$.

$$
\begin{aligned}
\Delta r & \simeq\left(B_{T} / B_{p}\right) \Delta\left(v_{\|} / \Omega\right), \\
& \simeq \Omega_{p}^{-1}\left(\Delta v_{\|}+v_{\|} a / R\right),
\end{aligned}
$$

where $\Omega_{p}=e B_{p} / m c$ is the poloidal gyrofrequency, $\Delta v_{\|}$ is to be determined from Eq. (3.19), and we have approximated, for large aspect ratio

$$
|\Delta B / B| \simeq \Delta R / R \simeq a / R \ll 1,
$$

where $a$ is the minor radius of the torus. Equation (3.36) further simplifies in the following two regions of phase space. (i) For the far untrapped particles with small $\mu$, Eq. (3.19) yields $\left|\Delta v_{\|}\right| \sim v_{\text {th }} a / R$, whence

$$
\Delta r \simeq \rho_{p}(a / R), \quad v_{\perp} \ll v_{\|},
$$

where $\rho_{p}$ is the gyroradius in the poloidal field. (ii) For the trapped and nearly trapped particles we find $\Delta v_{\|}$ $\sim v_{\text {th }}(a / R)^{1 / 2}$ and therefore

$$
\Delta r \simeq \rho_{p}(a / R)^{1 / 2}, \quad v_{\perp} \sim v .
$$

Hence these particles make the largest excursions. That the g.c. excursion is proportional to $\rho_{p}$, rather than $\rho$, was first noted by Tamm (1959).

The trapped particle g.c. orbits have been named banana orbits (Furth and Rosenbluth, 1969), for reasons evident from Fig. 2, and the trapped (or nearly trapped) particle excursion width

$$
\Delta_{b}=\rho_{p}(a / R)^{1 / 2}
$$

is called the banana width.

\section{Diffusion}

The preceding discussion neglects the microscopic Coulomb interaction between particles. Such "collisions" change each particle's energy and magnetic moment, causing diffusion in both velocity- and coordinatespace. As noted in Sec. II, the character of this diffusion depends upon the size of the collision frequency. 


\section{Collisional regime}

In the collision frequency range $\Omega \gg \nu_{c} \gg \omega$, free particle motion occurs only on the short time-scale of the gyroperiod. After a time $\nu_{c}^{-1}$, before a particle has traversed any appreciable distance along the field line about which it is gyrating, the particle's $\mu$ and $\epsilon$ will have diffused sufficiently for an effective $90^{\circ}$ scattering. This results in a random perpendicular displacement of the g.c. orbit by a distance $\rho$. The classical diffusion coefficient given by Eq. (2.79) then follows from the general random walk formula

$$
D=(\text { collision frequency }) \times(\text { step-size })^{2} .
$$

As noted previously, classical transport occurs in all collision-frequency regimes.

Of course the $\omega$-time-scale free particle orbits considered previously are not relevant in the collisional regime, in which the g.c. motion is predominantly stochastic. However, the $\delta \omega$-time-scale g.c. drift remains significant, in that the $\nabla B$ forces, like an electric field in a resistive medium, act to superimpose an overall drift motion on the g.c. random walk. To see how this $\mathrm{dr}$ ift leads to spatial diffusion, recall that it is radially inward for an electron (for example) in the lower half of the torus, and radially outward for an electron in the upper half. Let $\tau$ be the time for a g.c. to diffuse along a field line, half way around the magnetic axis; the g.c. then reverses the direction of its mean drift after each interval $\tau$, and thus performs a radial random walk with step-size $v_{D} \tau$ and effective collision frequency $\tau^{-1}$. The resulting diffusion coefficient is

$$
D \simeq v_{D}^{2} \tau
$$

We may estimate the diffusion time, $\tau$, by noting that the random walk along the field lines is characterized by a step-size $v_{\text {th }} / \nu_{c}$, the classical mean free path. Hence, after a time $t$, the g.c. will have diffused a distance $\left(v_{\mathrm{th}} / \nu_{c}\right)\left(\nu_{c} t\right)^{1 / 2}$; it will have traversed the magnetic axis when this distance is comparable to the "connection length" $q R$ (where $q$ is the safety factor and $R$ the major toroidal radius). Therefore $q^{2} R^{2}=\left(v_{\mathrm{th}}^{2} / \nu_{c}\right) \tau$; with $v_{D} \simeq \rho v_{\text {th }} / R$, Eq. (3.42) provides the Pfirsch-Schlüter coefficient

$$
D_{\mathrm{PS}}=q^{2} \rho^{2} \nu_{c}=q^{2} D_{c} .
$$

\section{Collisionless regimes}

Let $\Theta$ be the pitch angle of the gyro-orbit

$$
\sin ^{2} \Theta=2 \mu B / v^{2} \text {. }
$$

In a random walk description, the change in $\Theta$ due to Coulomb scattering is proportional to the square-root of the time. Since the classical collision frequency, $\nu_{c}$, pertains to roughly $90^{\circ}$ changes in $\Theta$, the collision frequency for some arbitrary change $\Delta \Theta$ is

$$
\nu(\Delta \Theta)=\nu_{c} /(\Delta \Theta)^{2} .
$$

For example, the collision frequency for scattering a magnetically trapped particle onto an untrapped orbit is roughly

$$
\nu_{t} \simeq(B / \Delta B) \nu_{c}
$$

where $\Delta B$ measures the field variation which causes the trapping, and we have noted, from Eq. (3.19), that the trapped particle region in velocity space is described by $\Delta \Theta \cong(\Delta B / B)^{1 / 2}$; see Fig. 3 .

In other words, a trapped particle remains on a given collisionless orbit only for a time (orbit correlation time) $\nu_{t}^{-1}$, so that trapped particle orbits are actually traversed only when

$$
\nu_{t}<\omega_{b},
$$

where $\omega_{b}$ is the bounce frequency of the collisionless trapped particle motion, i.e., the trapped particle velocity $(\Delta B / B)^{1 / 2} v_{\text {th }}$ divided by the connection length

$$
\omega_{b}=(\Delta B / B)^{1 / 2} v_{\text {th }} / q R \text {. }
$$

Similarly, the form of the untrapped orbits is pertinent only if

$$
\nu_{c}<\omega_{T},
$$

where

$$
\omega_{T} \equiv v_{\mathrm{th}} / q R
$$

is the untrapped particle transit frequency.

It follows that in the case $\Delta B \ll B$, as in a large aspect-ratio torus, two small-collision-frequency regimes may be distinguished (Galeev and Sagdeev, 1968): the banana, or collisionless regime, in which

$$
\nu_{c} / \omega_{T}<(\Delta B / B)^{3 / 2},
$$

and the plateau, or intermediate regime, in which

$$
(\Delta B / B)^{3 / 2}<\left(\nu_{c} / \omega_{T}\right)<1 \text {. }
$$

In certain geometries, a more detailed classification can be useful. For example, in a stellerator, there are two measures of $\Delta B$, due, respectively, to the helical and toroidal field perturbations. Furthermore, some stellerator studies (Galeev, et al., 1969) have considered regimes of even smaller collision frequency, in which $B \nu_{c} / \Delta B$ is comparable to the toroidal drift frequency $\omega_{D}=\vec{\nabla} \zeta \cdot \vec{v}_{D}$, associated with the periodic motion of trapped banana orbits (superbanana diffusion). However, with the neglect of such extremely collisionless effects, the classification outlined above is quite generally adequate [see, for example, Connor and Hastie (1974)].

We now estimate (neoclassical) diffusion coefficients for these two regimes, specializing first to the axisymmetric, large aspect ratio case.

In the banana regime, the dominant contribution to diffusion comes from the trapped and nearly trapped particles, whose g.c.'s make the largest radial excursions. In Fig. 4 we show two electron orbits, both of which pass through the point $P$. (Although banana orbits are depicted, the following argument applies equally to nearly trapped particles with small $v_{\|}$.) Because the orbits are being traversed in opposite directions, one lies inside the indicated flux surface and the other lies outside. It is clear that a reversal of the g.c. parallel velocity at $P$ induces a radial step of the orbit center by a distance $\sim \Delta_{b}$. From Fig. 3 , we see that pitch angle diffusion by as little as $\Delta \Theta \simeq(a / R)^{1 / 2}$ is sufficient for such reversals, which therefore occur with frequency $\nu_{t}$. Hence the trapped particle diffusion coefficient is 
$\nu_{t} \Delta_{b}^{2}$; since trapped particles comprise a fraction $(a / R)^{1 / 2}$ of all the particles, the banana diffusion coefficient is roughly

$$
\begin{aligned}
D_{b} & \simeq(a / R)^{1 / 2} \nu_{t} \Delta_{b}^{2}, \\
& \simeq(a / R)^{1 / 2} \nu_{c} \rho_{p}^{2},
\end{aligned}
$$

where the second form follows from Eqs. (3.40) and (3.46). The banana coefficient exceeds $D_{c}$ by as much as two orders of magnitude in typical tokamak devices, because $\rho_{p} \gg \rho$.

In the plateau regime, collisions are too frequent for undisturbed banana motion, but the collisionless g.c. motion of particles with small $v_{\|}$remains significant. Consider the class of "resonant" particles with $v_{\|}<v_{0}$, where the speed $v_{0}$ is to be determined (Stringer, 1970). From Eq. (3.45), the effective collision frequency for scattering particles out of this class is roughly

$$
\nu\left(v_{0}\right)=\left(v_{\text {th }} / v_{0}\right)^{2} \nu_{c} .
$$

If

$$
\nu\left(v_{\mathrm{o}}\right)>v_{\|} / q R,
$$

then resonant particles will suffer effective collisions before they are able to sample upper and lower halves of the torus. The uncompensated radial drift of such particles, between effective collisions, leads to a random walk with step-size $v_{D} / \nu\left(v_{0}\right)$. The corresponding diffusion coefficient is

$$
D=\left(v_{0} / v_{\text {th }}\right) \nu\left(v_{0}\right)\left[v_{D} / \nu\left(v_{0}\right)\right]^{2},
$$

since only a fraction $v_{0} / v_{\text {th }}$ of all the particles are resonant in this sense. Finally, Eqs. (3.54) and (3.55) imply $v_{0} / q R=\nu_{c}\left(v_{\mathrm{th}} / v_{0}\right)^{2}$, so that Eq. (3.56) reduces to the plateau diffusion coefficient

$$
D_{p}=v_{D}^{2} / \omega_{T},
$$

so-called because it it independent of $\nu_{c}$.

In experimentally realistic geometries true plateau behavior does not occur, because the necessary inequalities, (3.52), cannot simultaneously be satisfied very strongly unless the aspect ratio is extremely large. Thus, for typical aspect ratios, the diffusion coefficient increases monotonically with $\nu$, and only a mild flattening is observed in the regime of Eq. (3.52) (Hinton and Rosenbluth, 1973). The plateau argument nonetheless provides a useful estimate for this regime.

\section{Asymmetry}

An asymmetrizing magnetic field perturbation $b(\zeta) \ll B$ has no effect on the form of the diffusion coefficients, as estimated above, for the collisional or plateau regimes. However the banana formula, Eq. (3.53), depends upon the periodic nature of the collisionless orbits, which pertains only in the axisymmetric case. In general, we must consider particles trapped by local maxima of $b$. Because the g.c. orbits of such "localized" particles (Gibson and Mason, 1969) may be confined to the upper or lower halves of the torus, they drift away from the magnetic surface, between effective collisions, in a manner similar to resonant particles in the plateau regime. The effective collision frequency for localized particles is $\nu_{A}=(B / b) \nu_{c}$, their step-size is roughly

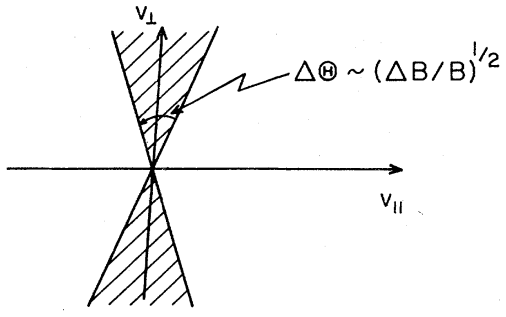

FIG. 3. Trapped and untrapped regions of velocity space, in terms of the parallel $\left(v_{\|}\right)$and perpendicular $\left(v_{\perp}\right)$ velocities. The trapped region is shaded; it occupies the region outside of a cone whose angle depends upon the field variation, $\Delta B$, as indicated.

$v_{D} / \nu_{A}$, and they comprise a fraction $(b / B)^{1 / 2}$ of all the particles. Hence our usual argument yields the diffusion coefficient

$$
\begin{aligned}
D_{A} & =(b / B)^{1 / 2} \nu_{A}\left(v_{D} / \nu_{A}\right)^{2} \\
& =(b / B)^{3 / 2} v_{D}^{2} / \nu_{c},
\end{aligned}
$$

appropriate to the banana regime

$$
\nu_{A}<\omega_{b}
$$

in an asymmetric system (Gibson and Mason, 1969; Galeev, et al., 1969; Frieman, 1970; Hazeltine and Rosenbluth, 1972; Connor and Hastie, 1974). As remarked previously, diffusion across the asymmetrical confining field of a stellerator is more complicated, in that several low collision frequency regimes can be distinguished. A detailed discussion of g.c. orbits and diffusion in stellerator geometry may be found in the review of Kadomtsev and Pogutse (1971), and in the review of Galeev and Sagdeev (1975).

A serious disadvantage of asymmetry, with regard to transport in a high temperature, nearly collisionless plasma, is evident from the $\nu_{c}^{-1}$ behavior of $D_{A}$.

\section{E. Distribution function}

\section{Velocity coordinates}

It is generally convenient to express the distribution function, $f$, in terms of the velocity variables $(\mu, \epsilon, \zeta)$, defined by Eqs. (3.1)-(3.4). Some technical properties of the transformation $\vec{v} \rightarrow(\mu, \epsilon, \zeta)$ are considered here.

First note that the variables $(\mu, \epsilon, \zeta)$ do not determine

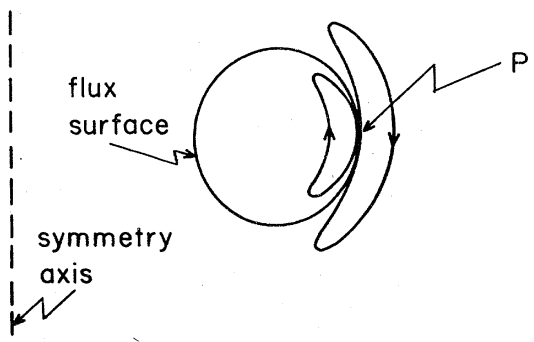

FIG. 4. Two banana orbits, both of which pass through the same point, $P$, on a flux surface, but which differ in the direction in which they are being traversed, at $P$. A smallangle collision at $P$, which causes a change in the sign of $v_{\|}$, leads to a radial step of the orbit center. 
$\overrightarrow{\mathrm{v}}$ uniquely, because the sign of $v_{\|}$is not specified [cf. Eq. (3.19)]. One must therefore introduce the additional, discrete variable

$$
\sigma \equiv v_{\|} /\left|v_{\|}\right| \text {. }
$$

Evidently $\sigma$ is a constant of the motion for untrapped particles, but not for trapped particles, whose parallel velocities change sign at each "bounce." The significance of this fact can be appreciated after the trapped and untrapped regions of velocity space have been more explicitly distinguished.

In general, the distinction between trapped and untrapped regions must be made relatively to a specific field line, about which a particle with given $(\mu, \epsilon)$ is gyrating. (The gyrophase $\zeta$ is clearly irrelevant to questions of trapping.) If $v_{\|}$, or $\epsilon-\mu B(\overrightarrow{\mathrm{x}})-e \Phi(\overrightarrow{\mathrm{x}}) / m$, can vanish for points $\vec{x}$ on a chosen field line, then $\mu$ and $\epsilon$ are in the trapped region relative to that field line. The points at which $v_{\|}$vanishes will be called bounce points, and denoted by $\vec{x}_{b}$. The set of all bounce points, corresponding to different field lines but to the same values of $\mu$ and $\epsilon$, is called the bounce surface and denoted by

$$
S_{b}(\mu, \epsilon) \equiv\{\overrightarrow{\mathbf{x}} \mid \epsilon-\mu B(\overrightarrow{\mathrm{x}})=e \Phi(\overrightarrow{\mathrm{x}}) / m\} .
$$

Since the value of $f$ at points $\overrightarrow{\mathrm{x}}_{b}$ on the bounce surface measures the number of particles being reflected at $\vec{x}_{b}$, we must have

$$
f\left(\overrightarrow{\mathbf{x}}_{b}, \mu, \epsilon, \sigma=+1\right)=f\left(\overrightarrow{\mathbf{x}}_{b}, \mu, \epsilon, \sigma=-1\right),
$$

for all $(\mu, \epsilon)$ in trapped regions. Equation (3.61) may be considered as a boundary condition, in the sense that, for fixed $\mu$ and $\epsilon$, the bounce surface constitutes a boundary of the domain of definition of $f$. Of course the $\sigma$ dependence of $f$ is not constrained in untrapped regions.

We have already noted [Eq. (2.36)] that the electrostatic potential is nearly constant along $\overrightarrow{\mathrm{B}}$. For particles with large ionic charge, $Z \gg 1$, the variation of potential energy along field lines remains important (Hazeltine, et al., 1974), but more typically,

$$
\overrightarrow{\mathrm{v}}_{\mathrm{gc}} \cdot \vec{\nabla}(e \Phi / m) \ll \overrightarrow{\mathrm{v}}_{\mathrm{gc}} \cdot \vec{\nabla}(\mu B) .
$$

In this case the kinetic energy is a lowest-order constant of the motion, and the variables $(\lambda, w, \zeta)$, where

$$
\lambda=\mu / w, \quad w=\epsilon-e \Phi / m=v^{2} / 2,
$$

are most convenient. Since

$$
v_{\|}=\sigma[2 w(1-\lambda B)]^{1 / 2},
$$

the trapped and untrapped velocity space regions are given by $\lambda_{m}>\lambda>\lambda_{c}$ and $0<\lambda<\lambda_{c}$, respectively. Here

$$
\lambda_{m}=[B(\overrightarrow{\mathrm{x}})]^{-1}
$$

is the largest $\lambda$ for which $f(\overrightarrow{\mathrm{x}}, \lambda, w)$ is defined, and the critical $\lambda$ for trapping is

$$
\lambda_{c}=B_{\max }^{-1},
$$

where $B_{\max }$ is a local maximum of $B$ occurring on a given field line.

As an example, we consider the axisymmetric geometry of Eq. (2.87), with $B \simeq B_{T}$. The ergodic property of field lines then implies that the distinction between trapped and untrapped regions is uniform on each flux surface, i.e., $\lambda_{c}$ depends only on $\Psi$. Explicitly,

$$
\lambda_{c}=R_{\min } / I,
$$

where $R_{\min }$ is the smallest distance between the symmetry axis and the flux surface under consideration. The size of the trapped region still depends upon position, through $\lambda_{m}$. The bounce surface, $S_{b}(\lambda)$, consists of points such that

$$
R=\lambda I \text {. }
$$

Finally, we take note of the Jacobians for the two velocity-coordinate systems presented above:

$$
\int d^{3} \overrightarrow{\mathrm{v}}=B \sum_{\sigma} \int d \mu d \epsilon d \zeta /\left|v_{\|}\right|
$$

where the integration domain is given by $e \Phi / m<\epsilon<\infty$, $0<\mu<(\epsilon-e \Phi / m) / B$; and

$$
\int d^{3} \overrightarrow{\mathrm{v}}=B \sum_{\sigma} \int d \lambda d w d \zeta w /\left|v_{\|}\right|,
$$

where the integration domain is given by $0<w<\infty$, $0<\lambda<\lambda_{m}$.

\section{Gyrophase dependence}

We now consider the dependence of $f$ upon the gyrophase angle $\zeta$. Recalling the gyrophase average of Eq. (3.13), we define a function $\tilde{f}$ by

$$
f(x, \mu, \epsilon, \zeta, t)=\bar{f}(\vec{x}, \mu, \epsilon, t)+\tilde{f}(\vec{x}, \mu, \epsilon, \zeta, t) .
$$

An approximate expression for $\tilde{f}$ is easily derived. We note that, in the presence of rapid gyration, $f$ can satisfy the assumed orderings,

$$
\partial \ln f / \partial t \ll \Omega, \quad|\vec{\nabla} \ln f| \ll \rho^{-1},
$$

only if it is nearly constant on the gyrotrajectory

$$
f(\overrightarrow{\mathrm{x}}, \mu, \epsilon, \zeta, t)=g(\overrightarrow{\mathrm{x}}-\vec{\rho}(\zeta), \mu, \epsilon, t),
$$

where $\vec{x}-\vec{\rho}=\vec{R}$, the position of the g.c., and $\vec{\rho}$ is given by Eq. (3.11). A first-order Taylor expansion of the function $g$ provides $\bar{f}=g(\overrightarrow{\mathbf{x}}, \mu, \epsilon, t)$ and $\tilde{f}=-\vec{\rho} \cdot \vec{\nabla} \bar{f}$. But $\bar{f}$ must be nearly Maxwellian, so we have

$$
\tilde{f}_{1}=-\vec{\rho} \cdot \vec{\nabla} f_{M}+O\left(\delta^{2}\right) .
$$

Later in this section, we outline the derivation, from kinetic theory, of the more accurate expression

$$
\begin{aligned}
\tilde{f}=-\vec{\rho} \cdot \vec{\nabla} \bar{f}+\vec{\rho} \cdot & {\left[\frac{\partial \bar{f}}{\partial \mu} \frac{1}{B}\left(\mu \vec{\nabla} B+v_{\|}^{2} \hat{n} \cdot \vec{\nabla} n+v_{\|} \frac{\partial \hat{n}}{\partial t}-\frac{e}{m} \overrightarrow{\mathrm{E}}\right)\right.} \\
& \left.+\frac{\partial \bar{f}}{\partial \epsilon} \frac{e}{m c} \frac{\partial \overrightarrow{\mathrm{A}}}{\partial t}\right] \\
+ & \frac{v_{\|} \mu}{\Omega} \frac{\partial \bar{f}}{\partial \mu}\left[\left(\hat{\rho} \hat{v}_{\perp}\right): \vec{\nabla} \hat{n}-\frac{1}{2} \hat{n} \cdot \vec{\nabla} \times \hat{n}\right],
\end{aligned}
$$

where $\vec{A}$ is the vector potential, $\hat{\rho}=\vec{\rho} / \rho$, and $\hat{v}_{\perp}=\vec{v}_{\perp} / v_{\perp}$.

It is instructive to compute the perpendicular particle flux from Eqs. (2.1) and (3.74)

$$
\int d^{3} \overrightarrow{\mathrm{v}} \overrightarrow{\mathrm{v}}_{\perp} f=\int d^{3} \overrightarrow{\mathrm{v}}_{\perp} \tilde{f}
$$

After considerable manipulation, using Eqs. (3.4), (3.61), and (3.69), one obtains 


$$
\int d^{3} \overrightarrow{\mathrm{v}}_{\perp} \tilde{f}=\int d^{3} \overrightarrow{\mathrm{v}}\left(\overrightarrow{\mathrm{v}}_{D}+\hat{\overrightarrow{\mathrm{v}}}_{\|}\right) \vec{f}-\vec{\nabla} \times \overrightarrow{\mathrm{M}}
$$

where $\overrightarrow{\mathrm{v}}_{D}$ and $\hat{\vec{v}}_{\|}$, given by Eqs. (3.17) and (3.16), are, respectively, the perpendicular and parallel g.c. drifts, and

$$
\overrightarrow{\mathrm{M}}=c \mathrm{P}_{\perp} \hat{n} / e B
$$

is the (single species) plasma magnetization. The g.c. flux, given by the first term on the right-hand side of Eq. (3.76), may be evaluated by substitution from Eqs. (3.16) and (3.17). Neglecting the $O\left(\delta^{4}\right)$ time derivative term, we obtain

$$
\begin{aligned}
\int d^{3} \overrightarrow{\mathrm{v}} \bar{f}\left(\overrightarrow{\mathrm{v}}_{D}+\hat{\overrightarrow{\mathrm{v}}}_{\|}\right)=c n \overrightarrow{\mathrm{E}} \times \overrightarrow{\mathrm{B}} / B^{2}+(c / e B) & \left\{\mathrm{P}_{\perp}(\hat{n} \times \vec{\nabla} B) / B\right. \\
& +\mathrm{P}_{\|}(\vec{\nabla} \times \hat{n})_{\perp} \\
& \left.+\mathrm{P}_{\perp}(\vec{\nabla} \times \hat{n})_{\|}\right\},
\end{aligned}
$$

where

$$
(\vec{\nabla} \times \hat{n})_{\perp}=\hat{n} \times[(\vec{\nabla} \times \hat{n}) \times \hat{n}]=\hat{n} \times \hat{n} \circ \vec{\nabla} \hat{n} .
$$

We interpret the perpendicular fluxes of Eqs. (3.76) and (3.78) by comparison with the rigorous flux, $n \overrightarrow{\mathrm{u}}_{\perp}$, obtained from the moment equation, Eq. (2.37). Through $O\left(\delta^{2}\right)$, the latter may be written as

$$
\begin{aligned}
n \overrightarrow{\mathrm{u}}_{\perp}= & c n \overrightarrow{\mathrm{E}} \times \overrightarrow{\mathrm{B}} / B^{2}+(c / e B)\left\{\mathrm{P}_{\perp}(\hat{n} \times \vec{\nabla} B) / B+\mathrm{P}_{\perp} \vec{\nabla} \times \hat{n}\right. \\
& \left.+\left(\mathrm{P}_{\|}-\mathrm{P}_{\perp}\right) \hat{n} \times(\hat{n} \cdot \vec{\nabla}) \hat{n}\right\} \\
& +n \overrightarrow{\mathrm{u}}_{c}-\vec{\nabla} \times \overrightarrow{\mathrm{M}},
\end{aligned}
$$

where $n \overrightarrow{\mathrm{u}}_{c}$ is the classical flux given by Eq. (2.77), and we have used Eq. (2.74) for the stress. From Eqs. $(3.78)-(3.80)$ we conclude

$$
\int d^{3} \overrightarrow{\mathrm{v}}\left(\overrightarrow{\mathrm{v}}_{D}+\hat{\overrightarrow{\mathrm{v}}}_{\|}\right) \bar{f}=n \overrightarrow{\mathrm{u}}_{\perp}-n \overrightarrow{\mathbf{u}}_{c}+\vec{\nabla} \times \overrightarrow{\mathrm{M}},
$$

and therefore, from Eq. (3.76)

$$
\int d^{3} \overrightarrow{\mathrm{v}}_{\perp} \tilde{f}=n \overrightarrow{\mathrm{u}}_{\perp}-n \overrightarrow{\mathrm{u}}_{c} .
$$

Hence the g.c. results, Eqs. (3.15) and (3.74), are not sufficiently accurate to describe classical collisional transport. This is not surprising, because the collision frequency does not appear explicitly in either $\mathrm{Eq}$. (3.15) or Eq. (3.74). The $O\left(\nu \delta^{2}\right)$ terms in $\tilde{f}$ which produce $n \tilde{\mathbf{u}}_{c}$ have been evaluated by Rosenbluth and Kaufman (1958), for the case of a uniform magnetic field. Alternatively, the classical transport coefficients may be evaluated from moments of the collision operator, Eqs. (2.77) and (2.84). In Sec. IV, we show that this method yields the same results, and requires knowledge of only the lowest-order $\tilde{f}$, given by Eq. (3.73).

On the other hand, neoclassical transport is fully included in Eqs. (3.81) and (3.82); the relevant collisional effects enter implicitly, through non-Maxwellian corrections to $\bar{f}$. In other words, while classical transport, which results from collisional interaction with the gyromotion, is neglected in Eq. (3.74) for $\tilde{f}$, neoclassical transport, which results from collisional interaction with the g.c. motion, can be included, in principle, by sufficiently accurate calculation of $\bar{f}$.
For the case of an axisymmetric system such refined calculation of $\bar{f}$ can be obviated by use of the generalized collisional moment prescription, Eq. (2.95), for which the first-order correction $\bar{f}_{1}=O(\delta)$ is sufficient. However, we shall see that this prescription is advantageous mainly in the banana regime. For arbitrary geometry and collision frequency, Eq. (3.81) provides the general formula for calculating neoclassical diffusion.

The g.c. flux differs from $n \overrightarrow{\mathbf{u}}_{\perp}$ also by the curl of the magnetization. This term is easily interpreted [see, for example, Spitzer (1967), p. 30ff.] in terms of the net circulation of gyrating particles; it is irrelevant to particle conservation, since $\vec{\nabla} \cdot \vec{\nabla} \times \vec{M}=0$. But the magnetization can enter the energy conservation law, as shown below.

Finally, it is instructive to consider the lowest-order flow. Substituting Eq. (3.73) into Eq. (3.75) we quickly find

$$
\int d^{3} \vec{v}_{\perp} \tilde{f}_{1}=n \vec{u}_{\perp 1},
$$

where the right-hand side is the diamagnetic (and electrostatic) drift given by Eq. (2.61), and we have recalled Eq. (2.36). Thus, as remarked in Sec. II, this firstorder flow is a result of Larmor gyration. Similarly,

$$
\int d^{3} \overrightarrow{\mathrm{v}}\left(\overrightarrow{\mathrm{v}}_{D}+\hat{\overrightarrow{\mathrm{v}}}_{\|}\right) f_{M}=n \overrightarrow{\mathrm{u}}_{\perp 1}+\vec{\nabla} \times \overrightarrow{\mathrm{M}}_{1},
$$

where $\overrightarrow{\mathrm{M}}_{1}=c p \hat{n} / e B$ is the lowest-order magnetization.

We next turn our attention to the energy flux, $\vec{Q}_{\perp}$. By manipulations similar to those used in deriving Eq. (3.81), we find

$$
\begin{aligned}
\int d^{3} \overrightarrow{\mathrm{v}} \bar{f}\left(m v^{2} / 2\right)\left(\overrightarrow{\mathrm{v}}_{D}+\hat{\overrightarrow{\mathrm{v}}}_{\|}\right) & =\overrightarrow{\mathrm{Q}}_{\perp}-\overrightarrow{\mathrm{Q}}_{c}+\vec{\nabla} \times\left(m c \overrightarrow{\mathrm{B}} \mathrm{R}_{\perp} / e B^{2}\right) \\
& -c \mathrm{P}_{\perp} \hat{n} \times \vec{\nabla} \Phi / B,
\end{aligned}
$$

where $\vec{Q}_{c}$ is the classical perpendicular energy flux, and $R_{\perp}$ is defined by Eqs. (2.4) and (2.75). The first three terms on the right-hand side of Eq. (3.85) are closely analogous to those on the right-hand side of Eq. (3.81), and only the last term, which apparently describes an electrostatic transport of energy, requires comment. It enters the energy conservation law through its divergence; notice that

$$
\vec{\nabla} \cdot c \mathrm{P}_{\perp} \hat{n} \times \vec{\nabla} \Phi / B=e \vec{\nabla} \Phi \cdot \vec{\nabla} \times \overrightarrow{\mathrm{M}},
$$

using Eq. (3.77). Hence the last term of Eq. (3.85) takes into account electrostatic energy changes, en $\overrightarrow{\mathrm{u}} \cdot \vec{\nabla} \Phi$, associated with the plasma magnetization. From Eq. (2.59) we observe that the lowest-order potential $\bar{\Phi}(\Psi)$ cannot contribute to the flux surface average of Eq. (3.86). In fact, in most theoretical treatments the poloidal variation of $\Phi$ turns out to be so small that the last term of Eq. (3.85) is omitted.

The lowest-order energy flux is

$$
\int d^{3} \overrightarrow{\mathrm{v}} \frac{m v^{2}}{2}\left(\overrightarrow{\mathrm{v}}_{D}+\hat{\overrightarrow{\mathrm{v}}}_{\|}\right) f_{M}=\overrightarrow{\mathrm{Q}}_{\perp 1}+\vec{\nabla} \times\left(\frac{5}{2} \frac{c}{e} \frac{p T \overrightarrow{\mathrm{B}}}{B^{2}}\right),
$$

where $\vec{Q}_{\perp_{1}}$ is given by Eq. (2.62), and we have recalled Eq. (2.29).

In conclusion, we have shown that the solution to the 
classical transport problem is given implicitly by Eqs. (2.77), (2.84), and (3.73). We have also derived general expressions for the neoclassical particle and energy fluxes, Eqs. (3.81), and (3.85), in terms of the gyrophase-averaged distribution function $\bar{f}$. In an idealized geometry with uniform magnetic field, $\bar{f}=f_{M}$, and the only radial transport is classical [cf. Eqs. (2.59), (2.63), (3.84), and (3.87)]. But in the necessarily nonuniform field of a toroidal confinement system, g.c. motion across the density and temperature gradients produces non-Maxwellian corrections to $\bar{f}$, which are typically first order in the poloidal gyroradius, and which yield neoclassical transport. Hence a fundamental task of transport theory is the solution of the drift-kinetic equation, which determines $\bar{f}$.

\section{Drift-kinetic equation}

The drift-kinetic equation is the Fokker-Planck equation, ordered in the gyroradius and averaged over the gyrophase. Formal derivations have been presented by, for example, Hastie, et al. (1967), Rutherford and Frieman (1968), Frieman (1970), Hazeltine (1973), and Glasser and Thompson (1973). The derivation outlined here follows the recursive procedure of Hazeltine (1973).

In terms of the velocity variables $(\mu, \epsilon, \zeta)$, Eq. (1.1) can be written as

$\frac{\partial f}{\partial t}+\left(\vec{v}_{\|}+\vec{v}_{\perp}\right) \cdot \vec{\nabla} f+\frac{\partial f}{\partial \mu} \frac{d \mu}{d t}+\frac{\partial f}{\partial \epsilon} \frac{d \epsilon}{d t}+\frac{\partial f}{\partial \zeta} \frac{d \zeta}{d t}=C(f ; f)$,

where $\overrightarrow{\mathrm{v}}_{\|}=\hat{n} \sigma\left|v_{\|}\right|$is given by Eq. (3.19), $\overrightarrow{\mathrm{v}}_{\perp}$ is given by Eq. (3.4), and $d \mu / d t, d \epsilon / d t$ and $d \zeta / d t$ are given by Eqs. (3.7)-(3.9). Note that all terms in Eq. (3.88) are at most of order $\omega=\delta \Omega$, except the $\Omega$ term in $d \zeta / d t$. To emphasize this, and to simplify notation, we introduce the operator $\vec{\delta}^{*}$, defined by

$$
\begin{gathered}
\vec{\delta} * f \equiv-\Omega^{-1}\left\{\frac{\partial f}{\partial t}+\frac{\partial f}{\partial \mu} \frac{d \mu}{d t}+\frac{\partial f}{\partial \epsilon} \frac{d \epsilon}{d t}-C(f, f)\right. \\
\left.+\frac{\partial f}{\partial \zeta}\left(\frac{d \zeta}{d t}-\Omega\right)\right\} .
\end{gathered}
$$

Then we have

$$
\partial f / \partial \zeta=\vec{\delta} * f
$$

which implies

$$
\overline{\bar{\delta} * f}=0
$$

and

$$
\frac{\partial f}{\partial \zeta}=\vec{\delta} * f-\overline{\vec{\delta} * f},
$$

where the overbar indicates a gyrophase average, as in Eq. (3.13).

At this point the small gyroradius approximation is introduced. Inferring from Eq. (3.89) that

$$
\tilde{f} \equiv f-\bar{f}=O(\delta),
$$

we replace Eq. (3.91) by

$$
\partial \tilde{f}^{(1)} / \partial \zeta=\vec{\delta} * \bar{f}-\overline{\vec{\delta} * \bar{f}},
$$

and Eq. (3.90) by

$$
\overline{\vec{\delta} *\left(\bar{f}+\tilde{f}^{(1)}\right)}=0 \text {. }
$$

The superscript on $\tilde{f}^{(1)}$ indicates that Eq. (3.92) results from Eq. (3.91) after one recursion; $\tilde{f}^{(1)}$ differs from a first-order solution to Eq. (3.91) in so far as we retain second- and higher-order terms in $\vec{\delta} * \bar{f}$.

Note that the collisional part of $\vec{\delta} *$ is bilinear, and involves the distribution functions $f_{s}$ for species other than that to which $f$ refers. In Eq. (3.92), we implicitly assume that $f_{s}$, like $f$, is replaced by its gyrophase average on the right-hand side. Then, since

$$
C\left(\bar{f}, \bar{f}_{s}\right)=\overline{C\left(\bar{f}, \bar{f}_{s}\right)},
$$

the collisional contribution to Eq. (3.92) vanishes. To obtain the $O\left(\nu \delta^{2}\right)$ terms in $\tilde{f}$, and thus to compute classical transport coefficients from Eq. (2.1), a second recursion is required. Since Eqs. (2.77) and (2.84) provide a much simpler prescription for classical transport, we do not consider the second recursion here.

Using Eqs. (3.7)-(3.9) and (3.20)-(3.21) for the righthand side, we can integrate Eq. (3.92) straightforwardly, to obtain the result given by Eq. (3.74). The general form of the drift-kinetic equation for $\bar{f}$ is obtained by substituting Eq. (3.74) into Eq. (3.93). Here, we restrict our attention to a simpler version, which is most pertinent to transport theory. First, we keep terms only through $O\left(\delta^{2}\right)$. Second, we assume

$$
\beta=O(\delta)
$$

so that Eqs. (3.22)-(3.24) are applicable. We then obtain

$$
\begin{array}{r}
\partial \bar{f} / \partial t+\left(v_{\|} \hat{n}+\overrightarrow{\mathrm{v}}_{d}\right) \cdot \vec{\nabla} \bar{f}+\frac{v_{\|} B}{\Omega} \hat{n} \cdot \vec{\nabla}\left(\frac{v_{\|} \hat{n} \cdot \vec{\nabla} \times \hat{n}}{B}\right) \mu \frac{\partial \bar{f}}{\partial \mu} \\
+(d \epsilon / d t)_{\mathrm{gc}} \partial \bar{f} / \partial \epsilon=C(\bar{f}, \bar{f}),
\end{array}
$$

where

$$
\begin{aligned}
(d \epsilon / d t)_{\mathrm{gc}} & \equiv \overline{d \epsilon / d t}+\mu \partial B / \partial t \\
& =(e / m) \partial \Phi / \partial t+\mu \partial B / \partial t-(e / m c) v_{\|} \hat{n} \cdot \partial \overrightarrow{\mathrm{A}} / \partial t,
\end{aligned}
$$

using Eq. (3.21).

Equation (3.95) is easily interpreted. The coefficient of $\nabla \bar{f}$ is the g.c. velocity; the coefficient of $\partial \bar{f} / \partial \mu$ displays the well-known (Hastie, et al., 1968) departure of $\mu$ from the exact, adiabatically invariant, magnetic moment; and the coefficient of $\partial \bar{f} / \partial \epsilon$, given by Eq. (3.96), is the rate of change of energy of a g.c. with magnetic moment $\mu$ and velocity $\overrightarrow{\mathrm{v}}_{\mathrm{gc}}=\overrightarrow{\mathrm{v}}_{\| \mid}[1+O(\delta)]$. Thus $\bar{f}$ appears in Eq. (3.95) as the phase-space distribution of g.c.'s.

To further simplify the equation, we begin by observing that the coefficient of $\partial \bar{f} / \partial \mu$ is second order in $\delta$, since $\hat{n} \cdot \vec{\nabla} \times \hat{n}=\hat{n} \cdot \vec{\nabla} \times \overrightarrow{\mathrm{B}} / B \sim \beta \sim \delta$. From consideration of the zeroth-order terms in Eq. (3.95),

$$
v_{\|} \hat{n} \cdot \vec{\nabla} \bar{f}_{0}=C\left(\bar{f}_{0}, \bar{f}_{0}\right),
$$

where the subscript refers to the $\delta$ ordering, it will be shown in Sec. $\mathrm{V}$ that $\overline{f_{0}}$ must be a Maxwellian, as anticipated in Eq. (2.27). Hence the $\partial \bar{f} / \partial \mu$ term is $O\left(\delta^{3}\right)$ and negligible.

With regard to the coefficient of $\partial \bar{f} / \partial \epsilon$, we first recall 
Eq. (2.31), which implies

$$
(e / m c) v_{\|} \hat{n} \cdot \partial \overrightarrow{\mathrm{A}} / \partial t=O\left(\delta \omega v_{\mathrm{th}}^{2}\right) .
$$

With regard to the $\partial B / \partial t$ term in Eq. (3.96), it must be noted that our previous estimate, Eq. (3.5), does not take into account the low- $\beta$ assumption of Eq. (3.94). Thus, restricting our attention to situations in which changes in the external current occur much more slowly than changes in the plasma current, and noting, from Eq. (2.40), that the plasma current contributes to $B$ only in $\operatorname{order} \beta$, we have

$$
\partial \ln B / \partial t \sim \beta \partial \ln p / \partial t=O\left(\beta \delta^{2} \omega\right) .
$$

Hence for second-order accuracy, the $\partial B / \partial t$ term in Eq. (3.96) may be neglected.

In axisymmetric systems, a more explicit and instructive derivation of Eq. (3.99) is possible. We observe from the equilibrium relation, Eq. (2.90), that

$$
B_{p}^{2}=|\nabla \psi / R|^{2} \sim P,
$$

whence

$$
\left(B_{p} / B_{T}\right)^{2}=O(\beta) \text {. }
$$

Since $B_{T}$, which is driven mainly by external currents, is nearly constant, we have

$$
\frac{\partial B}{\partial t}=B_{T} \frac{\partial}{\partial t}\left(1+B_{p}^{2} / B_{T}^{2}\right) \sim B \frac{\partial}{\partial t}\left(B_{p}^{2} / B_{T}^{2}\right),
$$

and the ordering of Eq. (3.99) follows. Equation (3.100) is often expressed in terms of the "poloidal $\beta$,"

$$
\beta_{p} \equiv 8 \pi P / B_{p}^{2} \sim 1 ;
$$

note that this provides an alternative explanation, for axisymmetric systems, of Eq. (2.23).

We have reduced Eq. (3.95) to the drift-kinetic equation

$$
\begin{aligned}
\partial \bar{f} / \partial t+ & \left(v_{\|} \hat{n}+\overrightarrow{\mathrm{v}}_{d}\right) \cdot \vec{\nabla} \bar{f} \\
+ & {\left[(e / m) \partial \Phi / \partial t-(e / m c) v_{\|} \hat{n} \cdot \partial \overrightarrow{\mathrm{A}} / \partial t\right] \partial \bar{f} / \partial \epsilon=C(\bar{f}, \bar{f}) . }
\end{aligned}
$$

This version provides the starting point for the kinetic theory of Secs. IV and V.

We conclude this section by considering the form that the drift-kinetic equation acquires when it is written in terms of the modified vector potential, $\vec{A}_{*}$. Neglecting the $\partial \bar{f} / \partial \mu$ term, we find from Eqs. (3.25), (3.26), and (3.95) that

$$
\partial \bar{f} / \partial t+\left(v_{\|} / B\right)\left[\overrightarrow{\mathbf{B}}_{*} \cdot \vec{\nabla} \bar{f}-\Omega\left(\partial \overrightarrow{\mathrm{A}}_{*} / \partial t\right) \partial \bar{f} / \partial \epsilon\right]=C(\bar{f}, \bar{f}) .
$$

A collisionless steady state is evidently described by $\overrightarrow{\mathrm{B}}_{*} \cdot \vec{\nabla} \bar{f}(\overrightarrow{\mathrm{x}}, \mu, \epsilon)=0$, the statement that $\bar{f}$ is constant on drift surfaces. For axisymmetric systems, we choose $\Psi_{*}$ of Eq. (3.31) as the drift-surface label; the collisionless steady state is then characterized by

$$
\bar{f}(\overrightarrow{\mathrm{x}}, \mu, \epsilon)=\mathfrak{F}\left(\psi-I v_{\|} / \Omega, \mu, \epsilon\right),
$$

where $\mathcal{F}$ is an arbitrary function of its arguments. For small poloidal gyroradius we may expand

$$
\bar{f}=\mathcal{F}(\psi, \mu, \epsilon)-\left(I v_{\|} / \Omega\right) \partial \mathcal{F} / \partial \psi+O\left(\delta^{2}\right),
$$

and by requiring Eq. (2.27) to hold, we find that

$$
\bar{f}=f_{M}(\psi, \epsilon)+g(\psi, \mu, \epsilon)-\left(I v_{\|} / \Omega\right) \partial f_{M} / \partial \psi,
$$

where the function $g$, which must be constant on flux surfaces, represents an $O(\delta)$ correction to the Maxwellian.

It turns out that Eq. (3.104) accurately describes the first-order distribution function even in the presence of collisions (Rutherford, 1970). Of course, solution of the first-order drift-kinetic equation, including collisions, is required to determine the function $g$.

The main content of Eq. (3.104) is that the first order distribution function can depend upon the poloidal angle $\Theta$ only through the $v_{\|} / \Omega$ factor appearing in the last term. In this sense, Eq. (3.104) is similar to Eqs. (2.66), (2.68), and (2.88) for the first-order flows, which were derived from consideration of the moment equations. Indeed, after substituting Eq. (3.104) into the definitions, Eqs. (2.1), (2.3), and (2.6), of the particle and heat fluxes, and using Eq. (3.69), we find that Eqs. (2.66) and (2.68) are reproduced; the flux functions $\hat{K}(\psi)$ and $\hat{L}(\psi)$ appear in this way as integrals of $g$ (Hazeltine, et al., 1973)

$$
\begin{aligned}
& \hat{K}(\psi)=2 \pi \sum_{\sigma} \sigma \int d \mu d \epsilon g, \\
& \hat{L}(\psi)=2 \pi \sum_{\sigma} \sigma \int d \mu d \epsilon m[\epsilon-e \Phi / m-(5 / 2) \bar{T} / m] g .
\end{aligned}
$$

The last term of Eq. (3.104)

$$
\begin{aligned}
\left(I v_{\|} / \Omega\right) \partial f_{M} / \partial \psi & \simeq\left(B_{T} R v_{\mathrm{th}}\right) /(\Omega|\nabla \psi|) \cdot\left|\nabla f_{M}\right| \\
& \sim \rho_{p}\left|\nabla f_{M}\right|,
\end{aligned}
$$

resembles the lowest-order $\tilde{f}$, as given by Eq. (3.73), with the important difference that the gyro-excursion $\rho$ is replaced by the radial g.c. excursion $\sim \rho_{p} \gg \rho$. Note that while $\rho_{p} \ll l$, the poloidal dimensions of the g.c. or bit, as shown in Fig. 2, are comparable to the dimensions of the confining system. That is, the collisionless g.c. orbit, unlike the gyro-orbit, carries a particle over macroscopic distances. It is for this reason that the neoclassical transport equations are not local: in general, only the flux surface average of, for example, the particle flux, can be related to corresponding averages of the density and temperature gradients. Neoclassical transport theory is radially local, of course, because the radial width of the excursion is presumed small. Finally, a radially local theory is sufficient because the basic macroscopic variables $(n$ and $T$ ) are nearly constant on flux surfaces.

\section{CLASSICAL AND COLLISION-DOMINATED TRANSPORT}

\section{A. Introduction}

In this section we begin the calculation of transport coefficients, restricting our attention to the collisional regime, in which the mean free path $v_{\text {th }} / \nu$ is much shorter than a macroscopic length $l$. The kinetic theory for this regime closely resembles the standard ChapmanEnskog theory (Chapman and Cowling, 1952), which also 
assumes $v_{\text {th }} / \nu \ll l$, and, in the sense described at the end of Sec. III, is nearly local. Thus the flux-surface geometry must be considered (to obtain the average particle or energy flux across a surface) only at the fluid, rather than at the kinetic, level. This partial decoupling of collisional and geometrical considerations is in sharp contrast to the kinetic theory for collisionless regimes, in which a relatively complicated, flux-surface-averaged, version of the drift-kinetic equation must be solved.

Hence the perpendicular transport problem for the collisional regime is comparatively simple, and only in this regime have exact transport coefficients, for arbitrary toroidal geometry, been calculated.

For convenience, we begin this section by calculating the classical perpendicular transport coefficients. However, it should be recalled that classical transport always occurs (so long as $\Omega \gg \nu$ ), and that the same classical results pertain in all collision frequency regimes.

We next consider, in Sec. IV.C, the classical parallel transport problem. While this topic has definite intrinsic interest, it would be beyond the subject of this review if it did not bear on the problem of neoclassical, perpendicular transport. Indeed, from the discussion of collisional transport in Sec. III, it is evident that the neoclassical (Pfirsch-Schlüter) enhancement of collision-dominated transport depends upon diffusion and heat conduction in the direction of the magnetic field.

Finally, in Sec. IV.D, we combine the results for the parallel transport problem with some conclusions of Sec. II, to obtain the neoclassical transport coefficients for a general toroidal geometry.

\section{B. Classical perpendicular transport}

We have already noted that, in a toroidal confinement system, classical transport typically presents only a small correction to neoclassical transport. Hence the following discussion, which is mainly intended to illustrate some remarks of previous sections, is brief, and only the major results are presented. More detailed discussions of classical transport may be found in, for example, the reviews of Kaufman (1966) and Braginskii (1965).

\section{Particle fluxes}

By our definition, Eq. (2.77), the classical perpendicular particle flux is proportional to the perpendicular friction force

$$
\overrightarrow{\mathrm{F}}_{\perp a}=\int d^{3} \overrightarrow{\mathrm{v}} m_{a} \overrightarrow{\mathrm{v}}_{\perp} C_{a} .
$$

Here

$$
C_{a}=\sum_{b} C_{a b}\left(f_{a}, f_{b}\right)
$$

is the bilinear Fokker-Planck collision operator, and $\overrightarrow{\mathrm{v}}_{\perp}$ is given by Eq. (3.4). Any momentum-conserving collision operator must satisfy

$$
\int d^{3} \overrightarrow{\mathrm{v}} m_{a} \overrightarrow{\mathrm{v}} C_{a a}\left(f_{a}, f_{a}\right)=0,
$$

since like-species collisions cannot change the bulk mo- mentum of that species. Thus only the unlike-species collision operator enters Eq. (4.1). For simplicity, we consider the case of a single ion species, and calculate the friction force exerted on the electrons

$$
\overrightarrow{\mathrm{F}}_{\perp e}=\int d^{3} \overrightarrow{\mathrm{v}} m_{e} \overrightarrow{\mathrm{v}}_{\perp} C_{e i}\left(f_{e}, f_{i}\right) .
$$

As noted in Sec. II, to compute the flux through $O\left(\delta^{2}\right)$, only the $O(\delta)$ form of $\overrightarrow{\mathrm{F}}_{\perp}$ is required. We therefore expand

$$
f_{a}=f_{a M}+f_{a 1}+\cdots,
$$

where $f_{a M}$ is a Maxwellian, as in Eq. (2.28), and $f_{1}=O(\delta)$ can be decomposed into its gyrophase-averaged and gyrophase-dependent parts

$$
f_{1}=\bar{f}_{1}+\tilde{f}_{1} \text {. }
$$

The corresponding expansion of the collision operator is written as

$$
C_{a b}\left(f_{a}, f_{b}\right)=C_{a b}\left(f_{a M}, f_{b M}\right)+C_{a b}^{l}\left(f_{1}\right)+O\left(\delta^{2}\right),
$$

where $C^{l}$ denotes the linearized operator

$$
C_{a b}^{l}\left(f_{1}\right)=C_{a b}\left(f_{a M}, f_{b 1}\right)+C_{a b}\left(f_{a 1}, f_{b M}\right) .
$$

The first term of Eq. (4.4) vanishes in general only if all plasma species have the same temperature

$$
C_{a b}\left(f_{a M}, f_{b M}\right)=\left(T_{a}-T_{b}\right) \mathfrak{e}(|\overrightarrow{\mathrm{v}}|) .
$$

The specific form of the function $\mathfrak{C}$, is not required here. We need only observe that, since the collision operator is rotationally symmetric-it does not involve a preferred direction in velocity space-and since the Maxwellians are similarly independent of direction, $\mathfrak{C}$ can depend upon only the magnitude of the velocity. Hence the first term of Eq. (4.4) cannot contribute to the integral of Eq. (4.3).

The rotationally symmetric property of the linearized collision operator implies that, for example,

$$
C^{l}(g \sin \zeta) \propto \sin \zeta,
$$

for any function $g$ which is independent of the gyrophase $\zeta$. Hence, after recalling Eq. (3.69), we see that the perpendicular friction force comes entirely from $\tilde{f}_{e 1}$ and $\tilde{f}_{i 1}$, and we have

$$
\overrightarrow{\mathbf{F}}_{\perp e}=\int d^{3} \overrightarrow{\mathrm{v}} m_{e} \overrightarrow{\mathrm{v}}_{\perp} C_{e i}^{\boldsymbol{l}}\left(\tilde{f}_{1}\right)+O\left(\delta^{2}\right) .
$$

Note, by a similar argument, that the parallel friction force involves only $\overline{f_{1}}$ :

$$
F_{\| l e}=\int d^{3} \overrightarrow{\mathrm{v}} m_{e} v_{\|} C_{e i}^{l}\left(\overline{f_{1}}\right)+O\left(\delta^{2}\right) .
$$

Equations (4.7) and (4.8), when considered together with Eqs. (2.94) and (2.95), make explicit the previously emphasized relation between classical transport, resulting from gyro-oscillation $(\tilde{f})$, and neoclassical transport, which results from g.c. motion $(\bar{f})$.

We next calculate $\overrightarrow{\mathrm{F}}_{\perp e}$ explicitly, using the lowestorder $\tilde{f}_{1}$ given by Eq. (3.73) and the small mass-ratio collision operator given by Eq. (1.16). After substituting the latter into Eq. (4.3), we obtain 


$$
\overrightarrow{\mathrm{F}}_{\perp e}=\frac{m_{e} \Gamma_{e} n_{i}}{2} \int d^{3} \overrightarrow{\mathrm{v}} \overrightarrow{\mathrm{v}}_{\perp}\left[\frac{\partial}{\partial \overrightarrow{\mathrm{v}}} \cdot \mathrm{U} \cdot \frac{\partial \tilde{f}_{e 1}}{\partial \overrightarrow{\mathrm{v}}}+\frac{2 m_{e}}{T_{e}} \frac{\overrightarrow{\mathrm{u}}_{i \perp} \cdot \overrightarrow{\mathrm{v}}}{v^{3}} f_{e M}\right]
$$

The first term here, coming from $C_{e i}\left(\tilde{f}_{e 1}, f_{i M}\right)$, may be simplified by integrating by parts, twice, and using the identity

$$
\partial / \partial \vec{v} \cdot u=-2 \vec{v} / v^{3} .
$$

The integral of the second term, which comes from $C_{e i}^{l}\left(f_{e M}, \tilde{f}_{i 1}\right)$, is straightforward to evaluate, because of the rotational symmetry of $f_{e M}$. Thus Eq. (4.9) reduces to

$$
\overrightarrow{\mathrm{F}}_{\perp e}=-\left(3 \pi^{1 / 2} / 4\right) m_{e} \tau_{e}^{-1} v_{\text {the }}^{2} \int d^{3} \overrightarrow{\mathrm{v}} \tilde{f}_{e 1} \overrightarrow{\mathrm{v}}_{\perp} v^{-3}+\left(m_{e} n_{e} / \tau_{e}\right) u_{i \perp} .
$$

Here

$$
\tau_{e}=\frac{3}{16 \pi^{1 / 2}} \frac{m_{e}^{2} v_{\text {the }}^{3}}{Z^{2} e^{4} n_{i} \ln \Lambda}
$$

is the electron-ion momentum exchange time (or "electron collision time") and $\overrightarrow{\mathrm{u}}_{i \perp}$ is the first-order ion perpendicular velocity, given by Eq. (2.61) (the 1-subscript is suppressed). From Eqs. (3.11) and (3.73) we have

$$
\tilde{f}_{e 1}=-\frac{\vec{v}_{\perp}}{\left|\Omega_{e}\right|} \cdot \hat{n} \times \vec{\nabla} f_{e M},
$$

where $\left|\Omega_{e}\right|=e B / m_{e} c(e>0)$, and the gradient to be taken at constant energy

$$
\nabla f_{e M}=f_{e M}\left[\nabla \ln p_{e}+\left(v^{2} / v_{\text {the }}^{2}-5 / 2\right) \vec{\nabla} \ln T_{e}-e \vec{\nabla} \Phi / T_{e}\right] .
$$

The integral in the first term of Eq. (4.11) is now readily evaluated, and after substitution from Eq. (2.61) we find

$$
\overrightarrow{\mathrm{F}}_{\perp e}=\frac{1}{\tau_{e}\left|\Omega_{e}\right|} \hat{n} \times\left[\vec{\nabla} P-\frac{3}{2} n_{e} \vec{\nabla} T_{e}\right],
$$

since $\Omega_{i}=-Z m_{e} \Omega_{e} / m_{i}$, for ions with charge $Z e$. Recall that $P=\sum_{a} p_{a}$ is the total pressure.

An important feature of Eq. (4.14) is that the electrostatic potential does not appear. Since this fact has not always been appreciated, we provide an alternate derivation. Suppose $\vec{\nabla} p_{e}=\vec{\nabla} p_{i}=\vec{\nabla} T_{e}=0$. Then the electron distribution function may be written as

$$
\tilde{f}_{e 1}=2 f_{e M} \vec{v}_{\perp} \cdot \overrightarrow{\mathrm{u}}_{\perp e} / v_{\text {the }}^{2},
$$

where

$$
\overrightarrow{\mathrm{u}}_{\perp e}=e \hat{n} \times \vec{\nabla} \Phi / m_{e}\left|\Omega_{e}\right| .
$$

Equation (4.15) gives the first-order form of a Maxwellian which is displaced in perpendicular velocity, i.e.,

$$
f_{M}(\overrightarrow{\mathrm{v}}-\overrightarrow{\mathrm{u}})=f_{M}(\overrightarrow{\mathrm{v}})\left[1+2 \overrightarrow{\mathrm{v}} \cdot \overrightarrow{\mathrm{u}} / v_{\mathrm{th}}^{2}+O\left(\delta^{2}\right)\right] .
$$

Furthermore, it can be seen that (for $\nabla p=0$ ) the two species are subject to the same displacement $\overrightarrow{\mathrm{u}}_{\perp e}=\overrightarrow{\mathrm{u}}_{\perp i}$ $\equiv \overrightarrow{\mathrm{u}}$. Hence the integrand of Eq. (4.7) involves the quantity

$$
C_{e i}\left(f_{e M}(\overrightarrow{\mathrm{v}}-\overrightarrow{\mathrm{u}}), f_{i M}(\overrightarrow{\mathrm{v}}-\overrightarrow{\mathrm{u}})\right)=C_{e i}\left(f_{e M}, f_{i M}\right) .
$$

Here we have noted that the collision operator must be Galilean invariant. Since, as noted previously, the right-hand side of Eq. (4.17) cannot contribute to the friction force, we have shown that the electrostatic potential cannot appear in the classical particle flux. This conclusion is evidently independent of the specific form of the collision operator, provided $C_{e i}$ is Galilean invariant and rotationally symmetric. In particular, the conclusion does not depend upon any small mass-ratio expansion.

After substituting Eq. (4.14) into Eq. (2.77), we obtain the classical perpendicular particle flux (Rosenbluth and Kaufman, 1958)

$$
\begin{aligned}
n \overrightarrow{\mathrm{u}}_{e c} & =-\left(m_{e} \Omega_{e}^{2} \tau_{e}\right)^{-1}\left(\vec{\nabla}_{\perp} P-\frac{3}{2} n_{e} \vec{\nabla}_{\perp} T_{e}\right), \\
& =n \overrightarrow{\mathbf{u}}_{i c} .
\end{aligned}
$$

The first term of Eq. (4.18) has been interpreted in Sec. II; a physical interpretation of the second, temperature gradient term may be found in the review of Braginskii (1965). It will be recalled that the ambipolarity condition of Eq. (4.19) follows from collisional momentum conservation.

\section{Energy fluxes}

According to Eqs. (2.10) and (2.84), the classical energy flux is proportional to the collision operator moment

$$
\overrightarrow{\mathrm{G}}_{\perp}=\int d^{3} \overrightarrow{\mathrm{v}}\left(m v^{2} / 2\right) \overrightarrow{\mathrm{v}}_{\perp} C^{l}\left(f_{1}\right) .
$$

As in the case of diffusion, only the linearized operator appears. But unlike the diffusion case, both like and unlike species collisions contribute. In particular, ion thermal conduction is dominated by the effects of ionion collisions; to lowest order in $\left(m_{e} / m_{i}\right)^{1 / 2}$, we have

$$
\overrightarrow{\mathrm{G}}_{\perp}=\int d^{3} \overrightarrow{\mathrm{v}}\left(m_{i} v^{2} / 2\right) \overrightarrow{\mathrm{v}}_{\perp} C_{i i}^{i}\left(\tilde{f}_{i 1}\right) .
$$

Since the like-particle collision operator must vanish when acting on a displaced Maxwellian [c.f. Eq. (4.17)], it is clear that only the temperature gradient term in $\tilde{f}_{i 1}$ contributes to $\overrightarrow{\mathrm{G}}_{\perp i}$ : to this order in the mass ratio, the classical ion energy flux is driven solely by the ion temperature gradient.

The corresponding electron moment, on the other hand,

$$
\overrightarrow{\mathrm{G}}_{\perp e}=\int d^{3} \overrightarrow{\mathrm{v}}\left(m_{e} v^{2} / 2\right) \overrightarrow{\mathrm{v}}_{\perp}\left[C_{e e}^{l}\left(\tilde{f}_{e 1}\right)+C_{e i}^{l}\left(\tilde{f}_{1}\right)\right]
$$

involves both like- and unlike-species collisions, so that both temperature and pressure gradient terms can be expected to enter the classical perpendicular energy flux. Of course the electrostatic potential gradient cannot enter, for precisely the same reasons that it disappears from the friction force.

The evaluation of the integrals in Eqs. (4.20) and (4.21) is lengthy, because of the complicated form of the likespecies collision operators. We present only the resulting fluxes, as given (in somewhat different forms) by Kaufman (1966) or Braginskii (1965)

$$
\vec{Q}_{i c}=-2\left(m_{i} \Omega_{i}^{2} \tau_{i}\right)^{-1} p_{i} \vec{\nabla}_{\perp} T_{i},
$$




$$
\overrightarrow{\mathrm{Q}}_{e c}=-\left(m_{e} \Omega_{e}^{2} \tau_{e}\right)^{-1}\left[0.91 p_{e} \vec{\nabla}_{\perp} T_{e}-T_{e} \vec{\nabla}_{\perp} P\right] .
$$

in Eq. (4.22), $\tau_{i}$ is the ion-ion collision time, defined by

$$
\tau_{i}=3 m_{i}^{1 / 2} T_{i}^{3 / 2} / 4 \pi^{1 / 2} Z^{4} e^{4} n_{i} \ln \Lambda .
$$

The numerical coefficient in the first term of Eq. (4.23) pertains only to the case of unit ionic charge; the value of this coefficient for other values of $Z$ may be found in Braginskii (1965).

Equations (4.18), (4.19), (4.22), and (4.23) complete the solution to the classical perpendicular transport problem. Notice that:

(i) The dominant cross-field transport process is ion thermal conduction. Indeed,

$$
\overrightarrow{\mathbf{Q}}_{i c} \sim\left(m_{i} / m_{e}\right)^{1 / 2} \overrightarrow{\mathbf{Q}}_{e c},
$$

while

$$
\overrightarrow{\mathrm{Q}}_{e c} \sim p_{e} u_{e c}=p_{e} u_{i c} \text {. }
$$

The ordering of Eq. (4.25) is not surprising, because we would expect $\vec{Q}_{i c} / \vec{Q}_{e c} \sim\left(\rho_{i}^{2} / \rho_{e}^{2}\right)\left(\tau_{e} / \tau_{i}\right)$, and Eqs. (2.18), (4.12), and (4.24) yield $\left(\rho_{i}^{2} / \rho_{e}^{2}\right)\left(\tau_{e} / \tau_{i}\right) \sim\left(m_{i} / m_{e}\right)^{1 / 2}$. How ever, the disparity between the ion energy flux and the other cross-field fluxes yields two distinct time scales for collisional dissipation; if $T_{e} \neq T_{i}$, some delicacy is required to obtain equations which simultaneously describe processes occurring on both time-scales. This matter is considered in detail in Sec. V.

(ii) to lowest order in the mass ratio, $\vec{Q}_{i c}$ involves only $\vec{\nabla} T_{i}$. The reason for this has been discussed previously. Of course there is a correction term, $\vec{Q}_{i c} *$ $\sim \vec{Q}_{e c}$, which involves the other gradients; to our knowledge, this correction has not been calculated.

It shall be seen that remarks similar to (i) and (ii) also apply to the neoclassical fluxes.

\section{Parallel transport}

\section{Kinetic equation}

The basic kinetic equations describing classical parallel transport can be derived from the Fokker-Planck equation (Braginskii, 1965), even in the absence of a magnetic field (Cohen, Spitzer and Routly, 1950). For reasons of consistency, our derivation proceeds from the drift-kinetic equation, Eq. (3.102).

All derivations are based upon an ordering in the Chapman-Enskog parameter-the mean free path divided by the scale length - which is denoted by

$$
\Delta=\omega / \nu \ll 1
$$

where $\omega=v_{\text {th }} / l$ is the transit frequency, and $\nu$ is the collision frequency. Of course small $\Delta$ corresponds to the collisional regime of neoclassical transport theory. The gyrophase-averaged distribution function is to be expanded in terms of the two small parameters, $\delta$ (the gyroradius parameter), and $\Delta$. Since only the firstorder terms in $\delta$ are needed here, we write

$$
\bar{f}=f_{M}+\overline{f_{1}},
$$

where $f_{M}$ is the usual Maxwellian, and $\overline{f_{1}}$ is further expanded

$$
\overline{f_{1}}=\sum_{n}{\overline{f_{1}}}^{n}, \quad{\overline{f_{1}}}^{n}=O\left(\Delta^{n} \delta\right) .
$$

From Eq. (3.97), we see that $f_{M}$ must be constant along field lines, so that parallel gradients of $n$ and $T$ enter the kinetic theory through $f_{1}$. To estimate the steepness of these gradients, we consider the parallel force balance law, Eq. (2.96). In the collision-dominated case, the stress is nearly isotropic, so we have

$$
\begin{aligned}
\hat{n} \cdot \vec{\nabla} p \equiv \nabla_{\|} p & =F_{\|}+e n E_{\|} \\
& =O\left(\delta \Delta^{-1}\right),
\end{aligned}
$$

because $F_{\|} \propto \nu$ [recall Eqs. (2.81) and (2.82)]. It follows that we should allow for an $n=-1$ term in the series of Eq. (4.28)

$$
\overrightarrow{f_{1}}={\overrightarrow{f_{1}}}^{-1}+{\overrightarrow{f_{1}}}^{0}+\cdots \text {. }
$$

For a maximal ordering, the parallel electric field term in Eq. (3.102) is also treated as $O\left(\delta \Delta^{-1}\right)$. More specifically, we assume

$$
(e / m c) v_{\|} \hat{n} \cdot \partial \overrightarrow{\mathrm{A}} / \partial t=O\left(\delta \Delta^{-1} \omega v_{\mathrm{th}}^{2}\right),
$$

as a refinement of Eq. (3.98). [In small collision frequency regimes, Eq. (4.32) is restrictive, and excludes electron runaway effects. See Sec. V.]

Turning our attention now to Eq. (3.102), we change the energy variable, for convenience, from $\epsilon$ to $w=\epsilon$ $-e \Phi / m$. This transformation yields

$$
\partial \bar{f} / \partial t+\left(v_{\|} \hat{n}+\vec{v}_{d}\right) \cdot \vec{\nabla} \bar{f}+e / m E_{\|} v_{\|} \partial \bar{f} / \partial w=C(\bar{f}, \bar{f}),
$$

where the time and space derivatives are taken at constant $\mu$ and $w$. The first-order version is evidently

$$
v_{\|} \hat{n} \cdot \vec{\nabla} \bar{f}_{1}-C^{l}\left(\bar{f}_{1}\right)=-\overrightarrow{\mathrm{v}}_{d} \cdot \vec{\nabla} f_{M}+e E_{\|} f_{M} / T,
$$

where $C^{l}$ is the linearized operator defined by Eq. (4.5). Before expanding Eq. (4.34) in $\Delta$, it is convenient to recall, from Sec. I, some properties of the linearized, unlike species, collision operators.

\section{Electron version}

The operator $C_{e i}^{l}$ is given, to lowest order in the mass ratio, by Eq. (1.16). When the distribution functions are independent of gyrophase, as in Eq. (4.33), this expression reduces to

$$
C_{e i}^{l}=\nu_{e i}(v)\left[\mathcal{L} f_{e 1}+2 v_{\|}\left(u_{i \|} / v_{\text {the }}^{2}\right) f_{e M}\right],
$$

where $\tau_{e}$ is given by Eq. (4.12)

$$
\nu_{e i} \equiv\left(3 \pi^{1 / 2} / 4 \tau_{e}\right)\left(v_{\text {the }} / v\right)^{3},
$$

and we have noted that

$$
\int d^{3} \overrightarrow{\mathrm{v}} \overrightarrow{\mathrm{v}} \overline{f_{i}}=n_{i} u_{i \|} \hat{n} \text {. }
$$

The operator $\&$ describes pitch-angle scattering of electrons by the much more massive ions. In terms of the cosine of the pitch angle

$$
\xi \equiv v_{\|} / v
$$

we have

$$
\mathscr{L}=\frac{1}{2}(\partial / \partial \xi)\left(1-\xi^{2}\right)(\partial / \partial \xi) .
$$


Equations (4.35)-(4.39) are written for the case of a single-ion species with charge $Z e$; however, if all ion species have (to sufficient accuracy) the same parallel flow velocity $u_{i \|}$, then the equations remain correct for any number of ion species, provided we make the replacement

$$
n_{i} Z^{2}-\sum_{i} n_{i} Z_{i}^{2}
$$

in Eq. (4.12). The quantity

$$
Z_{\mathrm{eff}}=\sum_{i} n_{i} Z_{i}^{2} / n_{e}
$$

is called the "effective $Z$."

Note that the electron-ion collision operator contains a term, proportional to $u_{i \|}$, which is independent of $f_{e 1}$. The convenient way to treat this inhomogeneous term is to introduce a function $g_{e}$, defined by

$$
\overline{f_{e 1}}=\frac{2 u_{i \|} v_{\|}}{v_{\text {the }}^{2}} f_{e M}+g_{e} \text {. }
$$

Equations (4.35) and (4.39) then imply

$$
C_{e i}^{l}\left(f_{1 e}\right)=\nu_{e i} \mathcal{L} g_{e} .
$$

Evidently $g_{e}$ represents the electron distribution function as measured in the rest frame of the ions [recall Eq. (4.16)]. Since

$$
C_{e e}\left(f_{e M}(\overrightarrow{\mathrm{v}}-\overrightarrow{\mathrm{u}}), f_{e M}(\overrightarrow{\mathrm{v}}-\overrightarrow{\mathrm{u}})\right)=0
$$

to all orders in $u / v_{\text {the }}$, we have

$$
C_{e e}^{l}\left(2 u_{\|} v_{\|} v_{\text {the }}^{-2} f_{e M}\right)=0 \text {. }
$$

We therefore introduce the notation

$$
C_{0 e}^{l} \equiv C_{e e}^{l}+\nu_{e i} \mathcal{L},
$$

i.e., $C_{0 e}^{l}$ is the homogeneous electron collision operator, corresponding to the case $u_{i}=0$. Equation (4.42) implies that

$$
C_{e}^{l}\left(f_{1 e}\right)=C_{0 e}^{l}\left(g_{e}\right),
$$

so that Eq. (4.34) becomes

$$
\begin{aligned}
v_{\|} \hat{n} \cdot \vec{\nabla} g_{e}-C_{0 e}^{l}(g)= & -e v_{\|} E_{\|} f_{e M} / T_{e}-\overrightarrow{\mathrm{v}}_{d} \cdot \vec{\nabla} f_{e M} \\
& -f_{e M} v_{\|} \hat{n} \cdot \vec{\nabla}\left(2 u_{i \|} v_{\|} / v_{\text {the }}^{2}\right) .
\end{aligned}
$$

After the function $g_{e}=O\left(\delta f_{e M}\right)$ is expanded in $\Delta$

$$
g_{e}=g_{e}^{-1}+g_{e}^{0}+\cdots,
$$

we find that Eq. (4.47) has one term of order $\Delta^{-2}$

$$
C_{0 e}^{l}\left(g_{e}^{-1}\right)=0 \text {. }
$$

This can be satisfied only by a perturbed Maxwellian

$$
g_{e}^{-1}=f_{e M}\left[\frac{p_{1}}{p_{0}}+\frac{T_{1}}{T_{0}}\left(\frac{v^{2}}{v_{\text {the }}^{2}}-\frac{5}{2}\right)+\frac{2 u_{e \|} v_{\|}}{v_{\text {the }}^{2}}\right] \text {. }
$$

With regard to the $O\left(\delta \Delta^{-1}\right)$ terms in Eq. (4.47), observe first that from Eq. (3.24)

$$
\overrightarrow{\mathrm{v}}_{d} \cdot \vec{\nabla} f_{M}=O\left(\delta \Delta^{0}\right)+O\left(\delta^{2} \Delta^{-1}\right) .
$$

Hence the g.c. drift is irrelevant in this order. The parallel flow terms would contribute if we assumed $u_{e \|} \sim u_{i \|} \sim \delta \Delta^{-1}$; in this way classical viscosity coefficients could be computed. However, for a toroidally confined, quiescent plasma, we show in Sec. $V$ that

$$
u_{i \|} \sim u_{e \|} \sim \delta \Delta^{0} \text {. }
$$

Therefore, for the purposes of this review, the parallel flow terms may be omitted; we thus obtain from Eq. (4.47)

$$
C_{0 e}^{l}\left(g_{e}^{0}\right)=f_{e M} v_{\|}\left(e E_{\|} / T_{e}+\hat{n} \cdot \nabla g_{e}^{-1}\right),
$$

which becomes, after substitution from Eq. (4.48),

$$
C_{o e}^{l}\left(g_{e}^{0}\right)=f_{e M} v_{\|}\left[A_{1}+\left(\frac{v^{2}}{v_{\text {the }}^{2}}-\frac{5}{2}\right) A_{2}\right] \text {. }
$$

Here the "forces" $A_{n}$, which drive the electron distribution away from equilibrium, are given by

$$
A_{1}=\nabla_{\|} \ln p_{e}+e E_{\|} / T_{e},
$$

and

$$
A_{2}=\nabla_{\|} \ln T_{e} .
$$

we have suppressed the subscripts of Eq. (4.48), with the understanding that $A_{n}=O\left(\delta \Delta^{-1}\right)$. The solution to Eq. $(4.50)$ is considered later.

\section{Ion version}

The collision operator describing the effect on the ion distribution function of scattering by electrons (or by relatively light ions) is given by Eq. (1.18). For small mass ratio and $\left|f_{i 1}\right| \sim\left|f_{e 1}\right|$, the dominant term is

$$
C_{i e}^{l}(\bar{f})=\left(F_{i \|} v_{\|} / p_{i}\right) f_{i M},
$$

where $F_{i \|}$ is the parallel component of the ion friction force. Evidently a transformation of the form of Eq. (4.41) is not useful for the ion kinetic equation, which is therefore written in terms of $f_{i 1}$. In other respects the reduction of the ion kinetic equation for parallel transport proceeds analogously to the electron case. In particular, the dominant perturbation to the ion distribution function, $f_{i 1}^{-1}$, has the form given by Eq. (4.48), so the $O\left(\delta \Delta^{-1}\right)$ version of Eq. (4.34) becomes, in this case

$$
\begin{aligned}
C_{i i}^{l}\left(\bar{f}_{i 1}^{o}\right)= & -C_{i e}^{l}\left(\bar{f}_{i 1}\right) \\
& +f_{i M} v_{\|}\left[\nabla_{\|} \ln p_{i}+\left(\frac{v^{2}}{v_{\mathrm{th} i}^{2}}-\frac{5}{2}\right) \nabla_{\|} \ln T_{i}-e E_{\|} / T_{i}\right],
\end{aligned}
$$

where the first term on the right-hand side is given by Eq. (4.53). Because of Eq. (4.29), this term cancels with the $E_{\|}$and $\nabla_{\|} p_{i}$ terms in Eq. (4.54), which therefore becomes

$$
C_{i i}^{l}\left(\bar{f}_{i 1}^{\mathrm{o}}\right)=f_{i M} v_{\|}\left(v^{2} / v_{\mathrm{th} i}^{2}-5 / 2\right) A_{i},
$$

with

$$
A_{i} \equiv \nabla_{\|} \ln T_{i} .
$$

This relation describes ion parallel heat conduction.

\section{Variational principle}

We have reduced the kinetic equations for both species to the general form

$$
C^{2}(f)=Q(v, \xi)
$$


where $C^{l}$ is a linearized, homogeneous, Fokker-Planck collision operator, $f$ is the function to be determined, and $Q$ is a given function of $v$ and $\xi=v_{\|} / v$. Because special cases of Eq. (4.57) were first solved by Spitzer and collaborators (Cohen, Spitzer and Routly, 1950; Spitzer and Härm, 1953), it is often referred to as the "Spitzer problem." In the special case of Eq. (4.50) with $\nabla_{\|} p_{e}$ $=\nabla_{\|} T_{e}=0$, the solution is called the Spitzer function, and denoted by $v_{\|} E_{\|} f_{S}$

$$
C_{o e}^{l}\left(v_{\|} f_{S}\right)=e v_{\|} f_{e M} / T_{e} \text {. }
$$

Spitzer's method of solution was based on numerical integration; the function $f_{s}$, tabulated by Spitzer and Härm (1953), is nearly exact.

Braginskii (1965) has solved Eq. (4.57) analytically, by expanding $f$ in a (truncated) series of Laguerre polynomials in $v$ (we shall see that the $\xi$-dependence of $f$ is readily determined, because of the rotational symmetry of $C^{l}$ ). This method is found to give quite accurate results, at least with regard to the relevant velocity moments of $f$.

A third method for solving the Spitzer problem, presented by Robinson and Bernstein (1962), is based on a variational principle. We outline the variational procedure here, because it resembles, in some respects, techniques used in neoclassical theory, and thus provides a useful introduction to the analysis of Sec. V. [See also Kaufman (1960).]

Since Robinson and Bernstein allowed for arbitrary relative magnitudes of the collision frequency and gyrofrequency, they considered a kinetic equation more complicated than Eq. (4.57). For a special case $\Omega \gg \nu$ of interest here, the variational principle is obtained as follows. For arbitrary functions $g_{1}(v, \xi)$ and $g_{2}(v, \xi)$, we define the functionals

$$
\begin{aligned}
& \mathcal{P}\left[g_{1}\right] \equiv-\int d^{3} \overrightarrow{\mathrm{v}}\left(g_{1} / f_{M}\right) Q, \\
& \mathcal{S}\left[g_{1}, g_{2}\right] \equiv-\int d^{3} \overrightarrow{\mathrm{v}}\left(g_{1} / f_{M}\right) C^{l}\left(g_{2}\right),
\end{aligned}
$$

and

$$
[\mathbb{R}] \equiv \mathfrak{Q}\left[g_{1}, g_{1}\right] \equiv \frac{\mathcal{P}^{2}\left[g_{1}\right]}{S\left[g_{1}, g_{1}\right]} .
$$

We denote the exact solution to Eq. (4.57) by $f$, and distinguish the functional evaluated at $f$ by omitting the brackets: $S \equiv S[f, f]$, etc. Note that Eq. (4.57) implies

$$
\mathcal{P}=\mathrm{S}=\boldsymbol{R} \text {. }
$$

The quantity $\$$ may be recognized as the rate of irreversible entropy production. Indeed, starting with the kinetic definition of entropy,

$$
S \equiv-\int d^{3} \overrightarrow{\mathrm{v}} f \ln f,
$$

retaining only the collisional term in $\partial f / \partial t$, and recalling Eq. (1.10), we find that

$$
(\partial S / \partial t)_{c}=-\int d^{3} \overrightarrow{\mathrm{v}} \ln f C(f) .
$$

We then expand $f=f_{M}+f_{1}$ and, for consistency with the small mass-ratio approximation of Eqs. (4.50) and
(4.55), omit the collisional energy exchange term, coming from $\ln f_{M}$. The result is $(\partial S / \partial t)_{c}=\delta$.

That the entropy production be positive (Boltzmann's " $H$-theorem") is guaranteed by the self-adjointness property

$$
\mathrm{s}\left[g_{1}, g_{2}\right]=\mathrm{s}\left[g_{2}, g_{1}\right] \text {. }
$$

Equation (4.63) is readily verified from the definitions of the operators $C_{i i}^{l}$ and $C_{e 0}^{l}$.

Now consider the first order variation of the function [ब]. Letting $g=f+\delta f$ in Eq. (4.61), and using Eq. (4.62), we find

$$
\delta[R]=\mathbf{2} \delta[\mathbb{P}]-\delta[S]
$$

where $\delta[\mathcal{P}]=\mathscr{P}[\delta f]$ and, according to Eq. (4.63)

$$
\delta S=2 S[\delta f, f] \text {. }
$$

It immediately follows from Eq. (4.57) that $[\mathbb{R}]$ is variational,

$$
\delta[\mathbb{R}]=0 .
$$

Furthermore, by considering the second-order variation of $[R]$, one can show that $[R] \leqslant R$, i.e., the variational principle is maximal.

It is instructive to evaluate $\mathcal{R}$ for the specific cases of Eqs. (4.50) and (4.55). Using Eqs. (4.41) and (5.62), we have, for the electron case,

$$
\begin{aligned}
& \mathbb{R}_{e}=\mathbb{P}_{e}=-\int d^{3} \overrightarrow{\mathrm{v}} v_{\|}\left[A_{1}+\left(\frac{v^{2}}{v_{\text {the }}^{2}}-\frac{5}{2}\right) A_{2}\right] \\
& \cdot\left(f_{e 1}-\frac{2 u_{i \|} v_{\|}}{v_{\text {the }}^{2}} f_{e M}\right) \\
&=A_{1} J_{\|} / e-A_{2} q_{\| e} / T_{e},
\end{aligned}
$$

where $J_{\|}$and $q_{\| e}$ are the parallel electric current and parallel electron heat flux, respectively, and we have recalled Eq. (2.30). Similarly, for the ion entropy production rate we find

$$
\mathfrak{R}_{i}=-A_{i} q_{\|_{i}} / T_{i} .
$$

Hence the two entropy production rates are given by the usual products of forces $\left(A_{1}, A_{2}, A_{i}\right)$ and fluxes $\left(-J_{\|} / e\right.$, $\left.q_{\| e} / T_{e}, q_{\| i} / T_{i}\right)$. Since the solutions to Eqs. (4.50) and (4.55) must be linear functions of the relevant forces, there must exist transport coefficients, $K_{m n}$, which linearly relate the fluxes to the forces. These coefficients are defined by

$$
\begin{aligned}
& J_{\|} / e=K_{11} A_{1}+K_{12} A_{2}, \\
& q_{\| e} / T_{e}=-K_{12} A_{1}-K_{22} A_{2}, \\
& q_{\| i} / T_{i}=-K_{i} A_{i} .
\end{aligned}
$$

Here we have taken note of the Onsager symmetry relation [see, for example, DeGroot and Mazur (1962)],

$$
K_{12}=K_{21} \text {, }
$$

which is implied by Eq. (4.63). Thus Eqs. (4.65) and (4.66) may be expressed as

$$
\begin{aligned}
& \mathfrak{R}_{i}=K_{i} A_{i}^{2}, \\
& \mathfrak{R}_{e}=K_{11} A_{1}^{2}+2 K_{12} A_{1} A_{2}+K_{22} A_{2}^{2} .
\end{aligned}
$$

To outline the variational procedure for approximate 
evaluation of the transport coefficients, we consider first the ion Spitzer problem given by Eq. (4.55). The solution, $f_{i 1}$ is represented by a trial function $f_{*}$; for example

$$
f_{*}=a_{1} v^{3}\left(1+a_{2} v^{2}\right) \xi,
$$

where $a_{1}$ and $a_{2}$ are variational parameters. We then use Eqs. (4.59)-(4.61) to express $\left[\mathbb{R}_{i}\right]$ in terms of the $a_{n}$; because the variational quantity is normalized, only $a_{2}$ finally appears

$$
\mathbb{R}_{i}\left[f_{*}, f_{*}\right]=\mathbb{R}_{i}\left(a_{2}\right) \text {. }
$$

After maximizing with respect to $a_{2}$, we obtain

$$
\mathbb{R}_{i *} \equiv \max _{a_{2}} \mathbb{R}_{i}\left(a_{2}\right)=K_{i *} A_{i}^{2} .
$$

Because the exact distribution function has in general a more complicated $v$ dependence than $f_{*}$, the coefficient $K_{i *}$ will differ from $K_{i}$. But because the quantity $\mathbb{R}_{i}\left(a_{2}\right)$ is variational, the difference must be second order in $f-f_{*}$ :

$$
K_{i *} \cong K_{i} \text {. }
$$

To our knowledge, the exact $K_{i}$ has not been calculated. Braginskii's expansion method yields the result

$$
K_{i}=3.9 n_{i} T_{i} \tau_{i} / m_{i} ;
$$

the variational procedure, using Eq. (4.73), reproduces this value precisely.

To evaluate a moment of $f_{*}$ other than $q_{\| i}$, the coefficient $a_{1}$ would be needed. This can be found from Eq. (4.62). Note, however, that the accuracy indicated in Eq. (4.75) need not apply when a trial function is used to calculate nonvariational quantities.

Of course similar remarks pertain to the electron Spitzer problem. After choosing a trial function $f_{* e}$, we calculate the maximal $R_{* e}$, as a quadratic form in $A_{1}$ and $A_{2}$. The variational transport coefficients $K_{m n *}$ may then be obtained by comparison with Eq. (4.72). In Table $\mathrm{I}$, the results of such a calculation are compared to those of Spitzer and Härm (1953), and Braginskii (1965); for this case, an electron trial function having the form given by Eq. (4.73) was used. The numbers $\kappa_{m n}$ listed in the table are related to the transport coefficients by

$$
K_{m n}=\left(\tau_{e} n_{e} T_{e} / m_{e}\right) \kappa_{m n} .
$$

Table II presents the Spitzer-Härm electron transport coefficients for several values of $Z_{\text {eff }}$. The classical parallel electrical conductivity is called the Spitzer conductivity, and denoted by $\sigma_{\|}$. Equation (4.67) shows that

$$
\sigma_{\|}=e^{2} K_{11} / T_{e}=\kappa_{\|} e^{2} \tau_{e} n_{e} / m_{e} .
$$

Finally, we note that the $\xi$ dependence of $f_{*}$, as given in Eq. (4.73), is exact. This can be seen by expanding $f$ and $Q$ in series of Legendre polynomials, $P_{n}(\xi)\left(P_{0}=1\right.$, $P_{1}=\xi$, etc.)

$$
f=\sum_{n} F_{n}(v) P_{n}(\xi), \quad Q=\sum_{n} Q_{n}(v) P_{n}(\xi) .
$$

Rotational symmetry of the collision operator implies

$$
C\left(F_{n} P_{n}\right) \propto P_{n},
$$

TABLE I. A comparison of the parallel transport coefficients, $\kappa_{m n}$, as evaluated by Spitzer and Härm (1953), by Braginskii (1965), and by the variational procedure outlined in the text. Only the case of $Z_{\text {eff }}=1$ is included.

\begin{tabular}{cccc}
\hline \hline & Spitzer-Härm & Braginskii & Variational \\
\hline$\kappa_{11}$ & 1.975 & 1.96 & 1.91 \\
$\kappa_{12}$ & 1.381 & 1.39 & 1.42 \\
$\kappa_{22}$ & 4.174 & 4.15 & 4.13 \\
\hline \hline
\end{tabular}

so that the various Legendre components of Eq. (4.57) decouple, and we need only solve

$$
C^{l}\left(F_{n} P_{n}\right)=Q_{n} P_{n}, \quad n=0,1, \ldots
$$

for $F_{n}$. Equations (4.50) and (4.55) involve only $Q_{1} P_{1}$, and therefore only $F_{1} P_{1}$, as in Eq. (4.73). (A $P_{2}$ component would appear, had we allowed for larger flow velocities.)

\section{Pfirsch-Schlüter transport}

Pfirsch and Schlüter (1962) first demonstrated the neoclassical enhancement of radial transport in the collisional regime $(\Delta \ll 1)$. This early treatment, based on previous work of Kruskal and Kulsrud (1958), used fluid equations, with an approximate model for the magnetic field geometry. Some temperature gradient effects were omitted. Similarly approximate results were later obtained from kinetic theory by, for example, Rutherford (1970) and Frieman (1970). The fluid treatment was revived by Hazeltine and Hinton (1973) who retained the temperature gradient terms and allowed for a general, axisymmetric field geometry. Equivalent results were later obtained by Bernstein (1974). Hence the argument presented here, which generalizes the Hazeltine-Hinton calculation to allow for asymmetry in the confining field, is close in spirit to the original Pfirsch-Schluter treatment.

\section{Radial fluxes}

We begin by deriving convenient expressions for the radial particle and energy fluxes. The analysis of the previous subsection shows that, through first order in $\delta$, each species in a collision-dominated plasma is described by a scalar pressure:

$$
P_{a}-1 p_{a}(\psi, \Theta, \zeta)=O\left(\delta^{2}\right), \text { for } \Delta \ll 1,
$$

where $I$ is a unit tensor and we recall

$$
\hat{n} \cdot \vec{\nabla} p_{a}=O\left(\delta \Delta^{-1}\right) \text {. }
$$

Similarly

$$
\mathrm{R}_{a}-1(5 / 2) p_{a} T_{a} / m_{a}=O\left(\delta^{2}\right) .
$$

TABLE II. The Spitzer-Härm transport coefficients $\kappa_{m n}$, for several values of the effective ionic charge $Z_{\text {eff }}$.

\begin{tabular}{lccrr}
\hline & $Z_{\text {eff }}=1$ & $Z_{\text {eff }}=2$ & $Z_{\text {eff }}=4$ & $Z_{\text {eff }}=\infty$ \\
\hline$\kappa_{11}$ & 1.98 & 2.32 & 2.67 & 3.39 \\
$\kappa_{12}$ & 1.38 & 2.05 & 2.91 & 5.16 \\
$\kappa_{22}$ & 4.17 & 6.75 & 10.01 & 20.32 \\
\hline \hline
\end{tabular}


These equations permit a straightforward generalization of Eqs. (2.93) and (2.100) to the non-axisymmetric case. Considering first the radial particle flux, we multiply the momentum conservation law, Eq. (2.15), by

$$
-(c / e) g^{1 / 2} \vec{\nabla} \psi \times \vec{\nabla} \Theta=-(c / e) g^{1 / 2} q^{-1} \overrightarrow{\mathrm{B}}_{T},
$$

where $g$ is the metric determinant of Eq. (2.43) and $q$ is the safety factor of Eq. (2.50). In view of Eq. (4.81), the stress tensor term is given to sufficient accuracy by

$$
\begin{aligned}
g^{1 / 2} \vec{\nabla} \psi \times \vec{\nabla} \Theta \cdot \vec{\nabla} p & =g^{1 / 2} \vec{\nabla} \psi \times \vec{\nabla} \Theta \cdot \vec{\nabla} \zeta \partial p / \partial \zeta \\
& =\partial p / \partial \zeta .
\end{aligned}
$$

The flux-surface average of this term vanishes because of Eq. (2.44), and, since,

$$
g^{1 / 2} \vec{\nabla} \psi \times \vec{\nabla} \Theta \cdot \overrightarrow{\mathbf{u}} \times \overrightarrow{\mathrm{B}}=\overrightarrow{\mathrm{u}} \cdot \vec{\nabla} \psi,
$$

we obtain the lowest-order radial particle flux

$$
\langle n \overrightarrow{\mathrm{u}} \cdot \vec{\nabla} \psi\rangle=-(c / e) q^{-1}\left\langle g^{1 / 2} \overrightarrow{\mathrm{B}}_{T} \cdot(\overrightarrow{\mathrm{F}}+e n \overrightarrow{\mathrm{E}})\right\rangle
$$

in close analogy to Eq. (2.93). The classical particle flux,

$$
\langle n \overrightarrow{\mathrm{u}} \cdot \vec{\nabla} \psi\rangle=-(c / e) q^{-1}\left\langle g^{1 / 2} \overrightarrow{\mathrm{B}}_{T} \cdot \overrightarrow{\mathrm{F}}_{\perp}\right\rangle,
$$

could be computed, for a given field geometry, from Eq. (4.14). We restrict our attention here to the neoclassical contribution, which is typically much larger. Recalling Eq. (2.67), which may be written as

$$
I(\psi, \Theta, \zeta)=q^{-1} g^{1 / 2} \overrightarrow{\mathrm{B}} \cdot \overrightarrow{\mathrm{B}}_{T},
$$

we find that Eq. (4.85) yields

$$
\left\langle n \overrightarrow{\mathrm{u}}_{\mathrm{NC}} \cdot \vec{\nabla} \psi\right\rangle=-(c / e)\left\langle I\left(F_{\|} / B+e n \overrightarrow{\mathrm{B}}_{T} \cdot \overrightarrow{\mathrm{E}} / \overrightarrow{\mathrm{B}}_{T} \cdot \overrightarrow{\mathrm{B}}\right)\right\rangle .
$$

Finally, we specialize to the electron version of Eq. (4.88). Parallel momentum balance [Eq. (4.29)] then requires $F_{\| l}=p_{e} A_{1}$, where $A_{1}$ is defined by Eq. (4.51), so we have

$$
\left\langle n \overrightarrow{\mathrm{u}}_{\mathrm{NC}} \cdot \vec{\nabla} \psi\right\rangle=(c / e) p_{e}\left\langle I A_{1} / B\right\rangle-c n_{e}\left\langle\overrightarrow{\mathrm{B}}_{T} \cdot \overrightarrow{\mathrm{E}} / \overrightarrow{\mathrm{B}}_{T} \cdot B\right\rangle .
$$

Here we have recalled Eq. (2.35).

A very similar argument yields, for the radial electron heat flux

$$
\left\langle\overrightarrow{\mathrm{q}}_{e \mathrm{NC}} \cdot \vec{\nabla} \psi\right\rangle=(5 / 2)(c / e) p_{e} T_{e}\left\langle I A_{2} / B\right\rangle,
$$

where $A_{2}=\hat{n} \cdot \vec{\nabla} T_{e} / T_{e}$, as in Eq. (4.52); and, for the radial ion heat flux

$$
\left\langle\overrightarrow{\mathrm{q}}_{i \mathrm{NC}} \cdot \vec{\nabla} \psi\right\rangle=-(5 / 2)(c / e) p_{i} T_{i}\left\langle I A_{i} / B\right\rangle,
$$

where $A_{i}=\hat{n} \cdot \vec{\nabla} T_{i} / T_{i}$.

One consequence of Eq. (4.85), of course, is that the lowest-order particle flux is automatically ambipolar (i.e., ambipolar for any electrostatic field), even in the presence of asymmetry, for $\Delta \ll 1$. This can also be seen from Eqs. (4.29) and (4.89), since the former implies

$$
\hat{n} \cdot \vec{\nabla} P=\hat{n} \cdot \vec{\nabla} \sum_{a} p_{a}=0,
$$

through $O\left(\delta \Delta^{-1}\right)$; thus the total pressure is more nearly uniform on each flux surface than are the pressures of the individual species. It follows that, in the case of a single ion species, Eqs. (4.89)-(4.91) provide all the radial fluxes of interest. The two ion species case, in which one species is much more massive than the other, can be treated by a closely analogous argument (Rutherford, 1974).

\section{Neoclassical transport coefficients}

In order to obtain transport equations from Eqs. (4.89)-(4.91), we must express the parallel gradients $\left(A_{1}, A_{2}\right.$ and $\left.A_{i}\right)$ in terms of radial gradients $(d P / d \psi$ and $d T / d \psi$ ) and the electric field. But our previous results make this task very simple. Consider first the neoclassical ion heat flux of Eq. (4.91). According to Eq. (4.69), the parallel ion heat flux is

$$
q_{\| i}=-T_{i} K_{i} A_{i},
$$

to lowest order in $\Delta$ and $\delta$. On the other hand, it was shown as Sec. II that this flux must have the general form

$$
q_{\| i}=-(5 / 2)(c / Z e B) I p_{i} d T_{i} / d \psi+\hat{L}_{i}(\psi) B .
$$

Therefore

$$
A_{i}=(5 / 2)(c / Z e B) K_{i}^{-1} I n_{i} d T_{i} / d \psi+\hat{L}_{i}(\psi) B / K_{i} T_{i} \text {. (4.93) }
$$

The function $\hat{L}_{i}(\psi)$ can now be determined by observing that Eqs. (2.53) and (4.56) require

$$
\left\langle A_{i} B\right\rangle=0 \text {; }
$$

thus

$$
A_{i}=(5 / 2)(c / Z e B) K_{i}^{-1} n_{i} d T_{i} / d \psi\left[I-B^{2}\langle I\rangle /\left\langle B^{2}\right\rangle\right] .
$$

Equations (4.76) and (4.91) then provide the neoclassical ion heat flux

$$
\begin{aligned}
\left\langle\overrightarrow{\mathrm{q}}_{i \mathrm{NC}} \cdot \vec{\nabla} \psi\right\rangle= & -1.6(c / Z e)^{2}\left(m_{i} / \tau_{i}\right) p_{i} d T_{i} / d \psi \\
& \cdot\left[\left\langle I^{2} / B^{2}\right\rangle-\langle I\rangle^{2} /\left\langle B^{2}\right\rangle\right] .
\end{aligned}
$$

The axisymmetric version of this result, as first obtained by Maschke (1972), differs only in that the $I^{2}$ factors can be taken outside the flux-surface average; recall Eq. (2.88).

The origin of the relation, Eq. (4.94), between $\nabla_{\|} T$ and $d T / d \psi$, can be traced back to our diffusion-time scale ordering, Eq. (2.20). Thus, for example, an excessive variation of $T_{i}$ along $\overrightarrow{\mathrm{B}}$, would induce an ion parallel heat flux which could not be consistent with Eq. (2.68), i.e., with the assumed equilibrium condition $\vec{\nabla} \cdot \overrightarrow{\mathrm{q}}_{i}=O\left(\delta^{2}\right)$.

Electron transport equations are obtained from Eqs. (4.89) and (4.90) in a similar way. Thus we combine Eqs. (2.70) and (2.68) of Sec. II

$$
\begin{aligned}
& J_{\|}=-c(I / B) d P / d \psi+K(\psi) B, \\
& q_{e \|}=(5 / 2)(c / e)\left(p_{e} / B\right) d T_{e} / d \psi+\hat{L}_{e}(\psi) B,
\end{aligned}
$$

with the electron parallel transport equations Eqs. (4.67) and (4.68). After the geometrical identities,

$$
\left\langle B A_{2}\right\rangle=0,\left\langle B A_{1}\right\rangle=e\left\langle E_{\|} B\right\rangle / T_{e},
$$

are used to eliminate $K(\psi)$ and $\hat{L}_{e}(\psi)$, we obtain

$$
\begin{aligned}
K_{11} A_{1}+K_{12} A_{2}= & -(c / e) d P / d \psi\left[I / B-B\langle I\rangle /\left\langle B^{2}\right\rangle\right] \\
& +\left(e K_{11} / T_{e}\right) B\left\langle E_{\|} B\right\rangle /\left\langle B^{2}\right\rangle,
\end{aligned}
$$




$$
\begin{aligned}
K_{12} A_{1}+K_{22} A_{2}= & -(5 / 2)(c / e) n_{e} d T_{e} / d \psi\left[I / B-B\langle I\rangle /\left\langle B^{2}\right\rangle\right] \\
& +\left(e K_{12} / T_{e}\right) B\left\langle E_{\|} B\right\rangle /\left\langle B^{2}\right\rangle .
\end{aligned}
$$

These equations can be solved for the $A_{n}$, in terms of the radial gradients and $\left\langle E_{\|} B\right\rangle$. When the results are substituted into Eqs. (4.89) and (4.90), we obtain the radial transport equations

$$
\begin{aligned}
\left\langle n \overrightarrow{\mathrm{u}}_{\mathrm{NC}} \cdot \vec{\nabla} \psi\right\rangle= & -(c / e)^{2}\left(m_{e} / \tau_{e}\right)\left[\left\langle I^{2} / B^{2}\right\rangle-\langle I\rangle^{2} /\left\langle B^{2}\right\rangle\right] \\
& \cdot\left[\left(\kappa_{22} / \kappa\right) d P / d \psi-\frac{5}{2}\left(\kappa_{12} / \kappa\right) n_{e} d T_{e} / d \psi\right] \\
& +c n_{e}\left[\langle I\rangle\left\langle E_{\|} B\right\rangle /\left\langle B^{2}\right\rangle-\left\langle I \overrightarrow{\mathrm{B}}_{T} \cdot \overrightarrow{\mathrm{E}} / \overrightarrow{\mathrm{B}}_{T} \cdot \overrightarrow{\mathrm{B}}\right\rangle\right],
\end{aligned}
$$

and

$$
\begin{aligned}
\left\langle\overrightarrow{\mathrm{q}}_{e \mathrm{NC}} \cdot \vec{\nabla} \psi\right\rangle= & -(5 / 2)(c / e)^{2}\left(m_{e} T_{e} / \tau_{e}\right) \\
& \cdot\left[\left\langle I^{2} / B^{2}\right\rangle-\langle I\rangle^{2} /\left\langle B^{2}\right\rangle\right] \\
& \cdot\left[(5 / 2)\left(\kappa_{11} / \kappa\right) n_{e} d T_{e} / d \psi-\left(\kappa_{12} / \kappa\right) d P / d \psi\right] .
\end{aligned}
$$

Here

$$
\kappa \equiv \kappa_{11} \kappa_{22}-\kappa_{12}^{2},
$$

and the $\kappa$ 's are given, for several values of ionic charge, in Table II. Again, the axisymmetric case is obtained by taking $I$ to be constant on flux surfaces.

Note also that Eqs. (4.67) and (4.96) provide the following expression for the parallel current

$$
J_{\|}=\sigma_{\|} B\left\langle E_{\|} B\right\rangle /\left\langle B^{2}\right\rangle-c d P / d \psi\left[I / B-\langle I\rangle B /\left\langle B^{2}\right\rangle\right] .
$$

The first term here gives the Spitzer current, as modified by toroidicity and $\nabla_{\|} P$ effects. Note than an electrostatic field cannot contribute, because of Eq. (2.53). The second term, called the Pfirsch-Schlüter or return current, is most easily understood in terms of the discussion of Sec. II.C.2: it maintains $\vec{\nabla} \cdot \vec{J}=0$ in the presence of the diamagnetic current. The return current cannot vanish (for nonuniform $B$ ), and will generally change sign across the plasma cross section.

Equation (4.98) can be seen to agree with the estimate of Sec. III,

$$
u_{\mathrm{NC}} \sim q^{2} u_{c}, \text { for } \Delta \ll 1,
$$

for large aspect-ratio, axisymmetric geometry. This case is considered in Sec. VI; it is convenient to anticipate some elements of the Sec. VI discussion here. With $B \simeq B_{T}=I / R$, the flux-surface averages in Eq. (4.98) reduce to

$$
\left\langle I^{2} / B^{2}\right\rangle-\langle I\rangle^{2} /\left\langle B^{2}\right\rangle \simeq\left\langle R^{2}\right\rangle-\left\langle R^{-2}\right\rangle^{-1} \simeq 2 r^{2},
$$

where $r$ is the minor radius of the flux surface, which is assumed to be nearly circular. Using $|\nabla \psi| \simeq B_{p} R$, we find that the flux $\overrightarrow{\mathrm{u}}_{\mathrm{NC}} \cdot \hat{r}$ is related to the density gradient $d n_{e} / d r$ by the Pfirsch-Schlüter diffusion coefficient

$$
D_{\mathrm{PS}}=\mathbf{2}\left(m_{e} \Omega_{e}^{2} \tau_{e}\right)^{-1}\left(\kappa_{22} / \kappa\right) q^{2}\left(T_{i}+T_{e}\right),
$$

where the safety factor $q$ is roughly given by $q=(r / R)\left(B_{T} / B_{p}\right)$, for this geometry. Referring to Eq. (4.18), we see that

$$
D_{\mathrm{PS}} / D_{c} \simeq 2\left(\kappa_{22} / \kappa\right) q^{2},
$$

as previously estimated. The original Pfirsch-Schlüter result ("Pfirsch-Schlüter factor") was slightly different, $D_{\mathrm{PS}} / D_{c}=2 q^{2}$, because the early studies did not properly allow for $\nabla_{\|} T_{e}$.

The large aspect-ratio, axisymmetric version of $\mathrm{Eq}$. (4.95) can be obtained in a similar way; this result was first derived by Shafranov (1965).

Finally, we note that the relation between the parallel and radial gradients, expressed by Eqs. (4.96) and (4.97), can (in the axisymmetric case) be used to obtain similar relations between the parallel and radial fluxes. In particular, the parallel current can be written in terms of the radial particle and heat fluxes (Hazeltine and Hinton, 1973). That such a relation also obtains, approximately, in other collision frequency regimes, was first pointed out by Ware (1973).

\section{KINETIC THEORY OF TRANSPORT IN AXISYMMETRIC SYSTEMS}

\section{A. Small gyroradius expansion}

The kinetic theory of transport in toroidal systems is based on a generalization of the well-known ChapmanEnskog method in the kinetic theory of gases (Chapman and Cowling, 1952), in which the motion along field lines is not necessarily localized by collisions. The basis of this kinetic theory is an expansion in powers of the poloidal gyroradius $\rho_{p}$, which measures the degree of localization in the direction perpendicular to a flux surface.

We begin by rewriting the drift-kinetic equation, Eq. (3.102), in terms of the independent variable $\bar{\epsilon} \equiv \epsilon$ $+(e / m)(\langle\Phi\rangle-\Phi)$ (instead of $\epsilon$ ) and $\mu$

$$
\frac{\partial f}{\partial t}+\left(\overrightarrow{\mathrm{v}}_{\|}+\overrightarrow{\mathrm{v}}_{d}\right) \cdot \vec{\nabla} f+\frac{e}{m}\left[\frac{\partial\langle\Phi\rangle}{\partial t}+v_{\|} E_{\|}\right] \frac{\partial f}{\partial \bar{\epsilon}}=C(f, f) .
$$

In axisymmetric systems the second term, $\overrightarrow{\mathrm{v}}_{\|} \cdot \vec{\nabla} f$, is of order $v_{\mathrm{th}}\left(B_{p} / B\right) / l$, while the third term $\overrightarrow{\mathrm{v}}_{d} \cdot \nabla f$, is of order $v_{\text {th }} \rho / l^{2}$, and smaller than the second by a factor $\rho_{p} / l$, where $\rho_{p} \equiv \rho B / B_{p}$ is the poloidal gyroradius, mentioned in Sec. II. We choose the basic small parameter to be $\delta_{p i} \equiv \rho_{p i} / l$, the ion poloidal gyroradius divided by a characteristic length (which may be the major or minor radius of the plasma). In this section, we use the assumption that $B_{p} / B \ll 1$, so that $\rho_{p} \gg \rho$, and we may expand the drift kinetic equation to any order in $\rho_{p}$.

We make a noncommittal choice of ordering for the like-particle collision terms, that these are of the same order as the term $\vec{v}_{\|} \cdot \vec{\nabla} f$. That is, the collision frequency is of the same order as the bounce or transit frequency. Subsidiary expansions will be carried out later for the limiting cases $\nu l / v_{\mathrm{th}}\left(B_{p} / B\right)$ small (Sec. V.D) or large (Sec. V.E) compared with unity. Before expanding $f$ in powers of $\delta_{p i}$, we must also choose the ordering of $E_{\|}$, and specify the collision terms in more detail. We make use of the fact that $\left(m_{e} / m_{i}\right)^{1 / 2}$ is small, in order to simplify the unlike particle collision terms.

\section{Electron-ion collision term}

We recall that the linearized electron-ion collision operator is given by Eq. (1.16), to lowest order in 
$\left(m_{e} / m_{i}\right)^{1 / 2}$. Only the gyrophase-averaged version will be needed here

$$
\bar{C}_{e i}^{l}=\nu_{e i}(v)\left[\mathcal{L} f_{e}+\left(2 v_{\|} u_{i \|} / v_{\text {the }}^{2}\right) f_{e M}\right],
$$

where the electron-ion collision frequency is

$$
\nu_{e i}(v) \equiv\left(3 \pi^{1 / 2} / 4 \tau_{e}\right)\left(v_{\text {the }} / v\right)^{3},
$$

with

$$
1 / \tau_{e}=\frac{4}{3}(2 \pi)^{1 / 2}\left(n_{i} Z_{i}^{2} e^{4} \ln \Lambda / m_{e}^{1 / 2} T_{e}^{3 / 2}\right)
$$

and $v_{\text {the }} \equiv\left(2 T_{e} / m_{e}\right)^{1 / 2}$. The pitch-angle scattering operator may be written either in terms of the cosine of the pitch angle $\xi\left(\equiv v_{\|} / v\right)$, or in terms of $\lambda(=\mu / w)$, where $\mu$ is the magnetic moment, and $w \equiv v^{2} / 2$

$$
\mathcal{L} \equiv \frac{1}{2} \frac{\partial}{\partial \xi}\left(1-\xi^{2}\right) \frac{\partial}{\partial \xi}=(2 \xi / B) \frac{\partial}{\partial \lambda} \lambda \xi \frac{\partial}{\partial \lambda} .
$$

\section{Ion-electron collision term}

The ion-electron collision term is given, to lowestorder in $m_{e} / m_{i}$, by Eq. (1.18). If $f_{i}$ and $f_{e}$ are Maxwellian (with different temperatures), this reduces to

$$
C_{i e}^{M}=\left(m_{e} n_{i} / m_{i} n_{i} \tau_{e}\right)\left(1-T_{e} / T_{i}\right)(\partial / \partial \vec{v}) \cdot\left(\vec{v} f_{i M}\right),
$$

which describes the temperature equilibration of the two species. By linearizing Eq. (1.18) and averaging over the gyrophase angle, we obtain the linearized operator

$$
\bar{C}_{i e}^{l}=v_{\|} F_{\| i} f_{i 0} / p_{i 0}+C_{i e}^{\prime},
$$

where $F_{\| i}$ is the friction force, and $C_{i e}^{\prime}$ is a term which will not be needed. With the further assumption given by Eq. (5.9), $C_{i e}^{\prime}$ is smaller than the first term in Eq. (5.7) by $O\left(\delta_{p i}\right)$.

\section{Maximal ordering}

The ordering of $E_{i \mid}$ and $\left(m_{e} / m_{i}\right)^{1 / 2}$ can be chosen by the following argument. We want the maximum number. of different processes, which determine the rate of change of $T_{\boldsymbol{e}}$ and $T_{\boldsymbol{i}}$, to be of the same order of magnitude. In particular, we do not want the temperature equilibration to occur on such a short time scale that we are forced to either set $T_{e}=T_{i}$ in the equations describing diffusion, or to include the transient contribution to the diffusion flux which is proportional to $T_{e}-T_{i}$. Such an effect was pointed out by Taylor (1961) in the context of classical diffusion.

The rate of change of ion temperature due to heat conduction is of order $\delta_{p i}^{2} / \tau_{i}$, while that due to equilibration is of order $m_{e} / m_{i} \tau_{e} \sim\left(m_{e} / m_{i}\right)^{1 / 2} / \tau_{i}$. Hence, we choose

$$
\left(m_{e} / m_{i}\right)^{1 / 2} \sim \delta_{p i}^{2} \text {. }
$$

The rate of change of electron temperature due to Joule heating is of order $n_{e} m_{e}\left(u_{e}-u_{i}\right)^{2} / \tau_{e}$, which is of the same order as the equilibration rate, $\left(m_{e} / m_{i} \tau_{e}\right) n_{e} T_{e}$, only if we choose

$$
\left|u_{e}-u_{i}\right| \sim\left(T_{e} / m_{i}\right)^{1 / 2} .
$$

Since $\left|u_{e}-u_{i}\right| \sim e E_{\|} \tau_{e} / m_{e}$, this implies

$$
E_{\|} / E_{\text {run }} \sim\left(m_{e} / m_{i}\right)^{1 / 2},
$$

where $E_{\mathrm{run}} \sim m_{\boldsymbol{e}} \dot{v}_{\text {the }} / e \tau_{\boldsymbol{e}}$ is the order of magnitude of the field which would cause massive electron runaway
(Dreicer, 1959). The order of magnitude of the electric field term in either the electron, or the ion, kinetic equation, relative to the collision term is thus $E_{\|} / E_{\text {run }}$ $\sim\left(m_{e} / m_{i}\right)^{1 / 2} \sim \delta_{p i}^{2} \sim \delta_{p e}^{2 / 3}$, [where we have used $\delta_{p e} \sim \delta_{p i}^{3}$, which follows from Eq. (5.8).] This may be treated as a term of order $\delta_{p e}$, along with the drift term, in the electron equation. In the ion equation, the $E_{\|}$and $F_{\|}^{e i}$ terms are both or order $\delta_{p i}^{2}$.

With the maximal ordering given by Eqs. (5.8) and (5.10), transport processes occur on two distinct time scales. (1) Joule heating, temperature equilibration, and ion heat conduction occur at roughly the same rate, $m_{e} / m_{i} \tau_{e}$, and are assumed to be approximately in balance, so that the temperatures change at a slower rate. (2) Particle diffusion, due to electron-ion collisions, and electron heat conduction, cause changes in the density and temperatures at the rate $\delta_{p e}^{2} / \tau_{e} \sim\left(m_{e} / m_{i}\right)^{3 / 2} / \tau_{e}$.

A complicating feature of the maximal ordering is that the rate $\delta_{p e}^{2} / \tau_{e}$, associated with the slow time scale, coincides with the rate $\delta_{p i}^{4} / \tau_{i}$, at which ion diffusion due to ion-ion collisions changes the ion density. Since the $O\left(\delta_{p i}^{4}\right)$ contribution to the ion particle flux is not included in Eq. (2.93), ambipolar diffusion is no longer guaranteed on the time scale of electron diffusion. Thus, with a maximal ordering the assumption of ambipolar diffusion must be made; in fact, as pointed out by Rosenbluth, et al. (1971), it determines the rate of change of plasma toroidal angular momentum. This process is considered in Sec. VI.C. [Note also that once ambipolarity is assumed, Eq. (2.93) is again valid.]

In the equal temperature case $\left(T_{i}=T_{e}\right)$ this complication is readily avoided, since $\left(m_{e} / m_{i}\right)^{1 / 2}$ need not be ordered in $\delta_{p i}$. This case might be most relevant to reactor-size experiments. The maximal ordering, however, has the advantages of describing present experiments more completely, and of yielding results which are easily specialized to the equal temperature case.

\section{Perturbation expansion}

By writing an expansion of the form

$$
f=f_{0}+f_{1}+f_{2}+\cdots
$$

for each species, where the different terms correspond to different powers of $\delta_{p}$, we obtain a sequence of equations from Eq. (5.1). The zeroth-order equations, obtained by keeping only the largest terms, are

$$
\begin{aligned}
& \overrightarrow{\mathrm{v}}_{\|} \cdot \nabla f_{e 0}=C_{e e}\left(f_{e 0}, f_{e 0}\right)+C_{e i}\left(f_{e 0}, f_{i 0}\right), \\
& \overrightarrow{\mathrm{v}}_{\|} \cdot \nabla f_{i 0}=C_{i i}\left(f_{i 0}, f_{i 0}\right),
\end{aligned}
$$

where $C_{e e}$ and $C_{i i}$ are the like-particle collision terms, given by Eq. (1.2), and $C_{e i}$ is the gyro-phase-averaged version of Eq. (1.14) (the overbar, indicating the average, will henceforth be omitted).

A generalization of the well-known $H$-theorem can be used to show that $f_{e 0}$ and $f_{i 0}$ must both be Maxwellian and constant on magnetic surfaces, as follows. We first multiply Eq. (5.11) by $\log f_{\text {eo }}$, and integrate over velocity space, making use of the Jacobian, Eq. (3.69). We obtain 


$$
\begin{aligned}
\overrightarrow{\mathrm{B}} \cdot \nabla \int d^{3} v & \frac{v_{\|}}{B}\left(f_{e 0} \log f_{e 0}-f_{e 0}\right) \\
= & \int d^{3} v \log f_{e 0} C_{e e}\left(f_{e 0}, f_{e 0}\right)+\int d^{3} v \log f_{e 0} C_{e i}\left(f_{e 0}, f_{i 0}\right)
\end{aligned}
$$

The usual $H$ theorem for the Fokker-Planck collision term (Lenard, 1960) shows that

$$
\int d^{3} v \log f_{e 0} C_{e e}\left(f_{e 0}, f_{e 0}\right) \leqslant 0,
$$

with equality only if $f_{e 0}$ is Maxwellian. Also, a straightforward integration by parts gives (assuming again $v \sim v_{\text {the }} \gg u_{i}$ )

$$
\begin{array}{r}
\int d^{3} v \log f_{e 0} C_{e i}\left(f_{e 0}, f_{i 0}\right)=-\frac{n_{i} \Gamma_{e}}{2} \int d^{3} w \frac{1}{f_{e 0}} \frac{\partial f_{e 0}}{\partial \overrightarrow{\mathrm{w}}} \\
\cdot v(\overrightarrow{\mathrm{w}}) \cdot \frac{\partial f_{e 0}}{\partial \overrightarrow{\mathrm{w}}} \leqslant 0,
\end{array}
$$

(where $\overrightarrow{\mathrm{w}} \equiv \overrightarrow{\mathrm{v}}-u_{i \|} \hat{n}$ ) with equality only if $f_{e 0}$ is isotropic in the ion mean rest frame (the reference frame moving with velocity $\overrightarrow{\mathrm{u}}_{i}$ relative to the laboratory). By averaging Eq. (5.13) over a flux surface, using Eq. (2.53), we have

$$
\left\langle\int d^{3} v \log f_{e 0}\left(C_{e e}+C_{e i}\right)\right\rangle=0,
$$

from which it follows that $f_{e 0}$ must be a Maxwellian, with mean velocity equal to that of the ions, $u_{i \|} \hat{n}$. The electron temperature may differ from the ion temperature, because Eq. (1.14) does not include the $O\left(m_{e} / m_{i}\right)$ temperature equilibration term. A similar analysis of Eq. (5.12) allows us to conclude that $f_{i_{0}}$ is also Maxwellian. Substitution into Eqs. (5.11) and (5.12) then shows that these Maxwellians must be constant along field lines (and hence constant on magnetic surfaces), at constant $\mu, \bar{\epsilon}$. It follows that $u_{i \|}=0$, to this order, since $\left(\overrightarrow{\mathrm{v}}-u_{i \|} \hat{n}\right)^{2}$ would otherwise contain $v_{\|}$, which is not constant along field lines, at constant $\mu, \bar{\epsilon}$. Hence,

$$
\begin{aligned}
& f_{e 0}=N_{e}\left(m_{e} / 2 \pi T_{e}\right)^{3 / 2} \exp \left(-m_{e} \bar{\epsilon} / T_{e}\right), \\
& f_{i 0}=N_{i}\left(m_{i} / 2 \pi T_{i}\right)^{3 / 2} \exp \left(-m_{i} \bar{\epsilon} / T_{i}\right),
\end{aligned}
$$

where $N_{e}=n_{e 0} \exp \left[-e\langle\Phi\rangle / T_{e}\right], N_{i}=n_{i 0} \exp \left[Z_{i} e\langle\Phi\rangle / T_{i}\right]$, with $n_{e 0}, n_{i 0}, T_{e}$, and $T_{i}$ functions of $\psi$ and $t$. Charge neutrality requires that $n_{e 0}=Z_{i} n_{i 0}$.

The first-order equations are

$$
\begin{aligned}
& \overrightarrow{\mathrm{v}}_{\|} \cdot \nabla f_{e 1}-\left(C_{e e}^{l}+C_{e i}^{l}\right) f_{e 1}=-\overrightarrow{\mathrm{v}}_{d e} \cdot \nabla f_{e 0}-\left(e / T_{e}\right) v_{\|} E_{\|} f_{e 0}, \\
& \overrightarrow{\mathrm{v}}_{\|} \cdot \nabla f_{i 1}-C_{i 1}^{l} f_{i 1}=-\overrightarrow{\mathrm{v}}_{d i} \cdot \nabla f_{i \mathrm{0}},
\end{aligned}
$$

where the superscript $l$ denotes the linearized collision operators. On the right-hand sides of these equations, the following expression for the drift velocity is to be used [recall Eq. (3.24) or Eq. (3.32)]

$$
\begin{aligned}
\overrightarrow{\mathrm{v}}_{d} \cdot \nabla \psi & =-v_{\|} \hat{n} \times \nabla\left(v_{\|} / \Omega\right) \cdot \nabla \psi, \\
& =I v_{\|} \hat{n} \cdot \nabla\left(v_{\|} / \Omega\right),
\end{aligned}
$$

where the spatial derivative acting on $v_{\|}$is taken at constant $\bar{\epsilon}, \mu$, neglecting the $O(\delta)$ term [contained in Eqs. (3.24) and (3.32)] due to the variation of $\Phi$ tangent to a flux surface. Then, since we only need the component of the drift perpendicular to flux surfaces, the derivative of $v_{\|}$may be taken at constant $w \equiv v^{2} / 2=\bar{\epsilon}$ $-(q / m)\langle\Phi\rangle$, i.e., we may use $v_{\|}=[2(w-\mu B)]^{1 / 2}$ or $v_{\|}=v(1-\lambda B)^{1 / 2}$, where $\lambda=\mu / w$. We also use $\lambda$ and $v$ as independent variables on the left-hand side of Eqs.

(5.19) and (5.20), which become

$$
\overrightarrow{\mathrm{v}}_{\|} \cdot \nabla f_{e 1}-C_{e}^{l} f_{e 1}=I \overrightarrow{\mathrm{v}}_{\|} \cdot \nabla\left(v_{\|} /\left|\Omega_{e}\right|\right) \frac{\partial f_{e 0}}{\partial \psi}-\left(e / T_{e}\right) v_{\|} E_{\|} f_{e 0},
$$

$$
\overrightarrow{\mathrm{v}}_{\|} \cdot \nabla f_{i 1}-C_{i i}^{l} f_{i 1}=-I \overrightarrow{\mathrm{v}}_{\|} \cdot \nabla\left(v_{\|} / \Omega_{i}\right) \frac{\partial f_{i 0}}{\partial \psi},
$$

where

$$
\frac{\partial f_{e 0}}{\partial \psi}=\left[\frac{\partial \ln n_{e 0}}{\partial \psi}-\frac{e}{T_{e}} \frac{\partial\langle\Phi\rangle}{\partial \psi}+\left(\frac{v^{2}}{v_{\text {the }}^{2}}-\frac{3}{2}\right) \frac{\partial \ln T_{e}}{\partial \psi}\right] f_{e 0},
$$

and

$$
\frac{\partial f_{i 0}}{\partial \psi}=\left[\frac{\partial \ln n_{i 0}}{\partial \psi}+\frac{Z_{i} e}{T_{i}} \frac{\partial\langle\Phi\rangle}{\partial \psi}+\left(\frac{v^{2}}{v_{\mathrm{th} i}^{2}}-\frac{3}{2}\right) \frac{\partial \ln T_{i}}{\partial \psi}\right] f_{i 0},
$$

with $v_{\text {th }} \equiv(2 T / m)^{1 / 2}$. Also, $C_{e}^{l} \equiv C_{e e}^{l}+C_{e i}^{l}$. The main problem of the kinetic theory is the solution of Eqs. (5.21) and (5.22).

The analogy with the Chapman-Enskog method is made complete by consideration of the constraints imposed by the second-order and third-order equations. By writing Eq. (5.1) to second and third order in $\delta_{p}$, and by the operation of integration over velocity space and averaging over a magnetic surface, we obtain solubility conditions in the form of surface-averaged moment equations. These equations determine the $\psi$ and $t$ dependence of $f_{e 0}$ and $f_{i 0^{\circ}}$. They will not be written down here, because we have already written down the (somewhat more accurate) moments of the exact Fokker-Planck equation, Eqs. (2.13) and (2.14), which need only to be averaged over a flux surface to yield the desired equations. This topic will be pursued in more detail in Sec. VII.

We now give a physical interpretation of some of the above equations. If the time derivatives were replaced in Eqs. (5.11) and (5.12), their solutions would approach the Maxwellian steady states, given by Eqs. (5.17) and (5.18), in a time of the order of the $90^{\circ}$-scattering collision time. The decay of small departures from these steady states, is described by linear equations. In the short mean-free-path limit, this decay is related to the classical parallel transport processes, considered in Sec. IV. Thus heat conduction makes the temperatures uniform on a magnetic surface, resistivity reduces the relative parallel flow velocity to zero, and parallel viscosity eliminates the parallel mass motion through regions of spatially varying magnetic field strength.

A similar interpretation of the time-dependent version of Eq. (5.20) can be given if the ion temperature gradient is zero. In this special case, the steady-state solution is

$$
f_{i 1}=\frac{2 v_{\|} u}{v_{\text {thi }}^{2}} f_{i 0},
$$

where 


$$
u \equiv-I\left(v_{\mathrm{th} i}^{2} / 2 \Omega_{i}\right)\left[\frac{\partial \ln n_{i 0}}{\partial \psi}+\frac{Z_{i} e}{T_{i}} \frac{\partial\langle\Phi\rangle}{\partial \psi}\right] .
$$

The relaxation of $f_{i 1}$, to this shifted Maxwellian, can be described in terms of the decay of poloidal rotation. Note that when Eq. (5.26) is used for the parallel velocity component and combined with Eq. (2.61) for the perpendicular component, one obtains zero for the poloidal component $\overrightarrow{\mathrm{u}}_{i} \cdot \overrightarrow{\mathrm{B}}_{p}=0$. The rate of decay of the poloidal velocity is of the order of the ion-ion $90^{\circ}$-collision time. In the collisional and plateau regimes, investigated by Stix (1973), the dissipation mechanisms are magnetic pumping (parallel viscosity) and Landau damping. In the banana regime, Rosenbluth (1973) has obtained a similar decay rate, due to friction between trapped and untrapped ions.

\section{Second-order equations}

Although we are primarily interested in the firstorder equations, the second-order ion equation is of some interest:

$$
\begin{aligned}
\overrightarrow{\mathrm{v}}_{\|} \circ \nabla f_{i 2}-C_{i i}^{l} f_{i 2}= & -\overrightarrow{\mathrm{v}}_{d i} \cdot \nabla f_{i 1}+v_{\|}\left(n_{i 0} Z_{i} e E_{\|}+F_{\| i}\right) f_{i 0} / p_{i 0} \\
& +C_{i i}\left(f_{i 1}, f_{i 1}\right) \\
& +\left(m_{e} n_{e} / m_{i} n_{i} \tau_{e}\right)\left(1-T_{e} / T_{i}\right) \\
& \times \frac{1}{v^{2}} \frac{\partial}{\partial v}\left(v^{3} f_{i 0}\right)-\left(\partial f_{i 0} / \partial t\right)
\end{aligned}
$$

The time derivative of $f_{i 0}$ is here taken at constant $v$, and is to be evaluated using the solubility conditions, to this order

$$
\begin{aligned}
& \partial n_{i 0} / \partial t=0, \\
& \begin{aligned}
\frac{3}{2} n_{i 0} \frac{\partial T_{i}}{\partial t}+\left\langle\nabla \cdot \int d^{3} v \frac{m_{i} v^{2}}{2} \overrightarrow{\mathrm{v}}_{d i} f_{i 1}\right\rangle & \\
& =3\left(m_{e} / m_{i} \tau_{e}\right) n_{e 0}\left(T_{e}-T_{i}\right),
\end{aligned}
\end{aligned}
$$

[where the second term may be rewritten, using Eq. (2.58)]. Thus, the temperature equilibration term cancels, and we have

$$
\begin{aligned}
\overrightarrow{\mathrm{v}}_{\|} \cdot \nabla f_{i 2}-C_{i i}^{l} f_{i 2}= & -\overrightarrow{\mathrm{v}}_{d i} \cdot \nabla f_{i 1} \\
& +\left(2 / 3 p_{i 0}\right)\left(v^{2} / v_{\mathrm{thi}}^{2}-\frac{3}{2}\right) f_{i 0} \\
& \times\left\langle\nabla \cdot \int d^{3} v \frac{m_{i} v^{2}}{2} \overrightarrow{\mathrm{v}}_{d i} f_{i 1}\right\rangle \\
& +v_{\|}\left(n_{i 0} Z_{i} e E_{\|}+F_{\| i}\right) f_{i 0} / p_{i 0}+C_{i i}\left(f_{i 1}, f_{i 1}\right),
\end{aligned}
$$

This equation has been solved in limiting cases, which are mentioned in Sec. VI.C.

It is instructive to consider here the version of Eq. (5.27) which is obtained when the ion temperature gradient is relatively small

$$
\partial \ln T_{i} / \partial \ln n_{i}=O\left(\delta_{p i}\right) \text {. }
$$

Then $f_{i 1}$ is given by the shifted Maxwellian of Eq. (5.25), and it is convenient to express $f_{i 2}$ in terms of a secondorder shifted Maxwellian

$$
f_{i 2}=\left(2 v_{\|}^{2} u^{2} / v_{\text {thi }}^{4}\right) f_{i 0}+g_{i 2} \text {. }
$$

Galilean invariance, as in Eq. (4.17), may now be used to simplify the collisional terms in Eq. (5.27), which becomes

$$
\begin{aligned}
\overrightarrow{\mathrm{v}}_{\|} \cdot \vec{\nabla} g_{i 2}-C_{i i}^{l} g_{i 2}= & -I \overrightarrow{\mathrm{v}}_{\|} \cdot \vec{\nabla}\left(v_{\|} / \Omega_{i}\right)\left(\frac{v^{2}}{v_{\mathrm{th} i}^{2}}-\frac{3}{2}\right) \frac{\partial \ln T_{i}}{\partial \psi} f_{i 0} \\
& +v_{\|}\left(n_{i 0} Z_{i} e E_{\|}+F_{\| i}\right) f_{i 0} / p_{i 0} \\
& -2 I\left(v_{\|} / B\right) \overrightarrow{\mathrm{v}}_{\|} \\
& \cdot \nabla\left(v_{\|} / \Omega_{i}\right)\left(f_{i 0} / v^{2}{ }_{i}\right) \partial(u B) / \partial \psi,
\end{aligned}
$$

where $u$ is given by Eq. (5.26). The first term on the right-hand side of the equation for $g_{i_{2}}$ comes from $\overrightarrow{\mathrm{v}}_{d} \cdot \vec{\nabla} f_{i 0}$, and appears in the second-order theory when the ion temperature gradient is small. The second term, containing the electric field and frictional coupling terms, is considered below. The third term, involving $\partial u B / \partial \psi$, is due to the gradient of the toroidal angular velocity. Recalling that $\overrightarrow{\mathrm{u}}_{i} \cdot \overrightarrow{\mathrm{B}}_{p} \simeq 0$, for small ion temperature gradient, we see that $u$ is related to the toroidal velocity $u_{T}$ by $u=\left(B_{T} / B\right) u_{T}$, and hence to the toroidal angular velocity $\omega_{T}=u_{T} / R$ by $u B=I \omega_{T}$. We do not consider the effects of this third term, since they have not been considered in the literature. We thus assume that $\partial(u B) / \partial \psi=0$; using Eq. (5.26) and neglecting $\partial I / \partial \psi$ $[=O(\beta)]$, this condition may be written as

$$
\partial \ln n_{i 0} / \partial \psi+\left(Z_{i} e / T_{i}\right) \partial\langle\Phi\rangle / \partial \psi=\text { const. },
$$

which corresponds to rigid body rotation: $\partial \omega_{T} / \partial \psi=0$.

The $E_{\|}$and $F_{\|}$terms in Eq. (5.27) are of particular importance, especially in the case of two ion species, which is considered later. We therefore find it convenient to treat these terms as first order, so that Eq. (5.22) becomes

$$
\begin{aligned}
\overrightarrow{\mathrm{v}}_{\|} \cdot \nabla f_{i 1}-C_{i i}^{\boldsymbol{l}}\left(f_{i 1}\right)= & -\boldsymbol{I} \overrightarrow{\mathrm{v}}_{\|} \circ \nabla\left(v_{\|} / \Omega_{i}\right) \partial f_{i 0} / \partial \psi \\
& +v_{\|}\left(n_{i 0} Z_{i} e E_{\|}+F_{\| i}\right) f_{i 0} / p_{i 0} .
\end{aligned}
$$

This artifice makes it easy to obtain results for the two ion species case, from the solution to the single ion species problem now under consideration.

\section{B. Linearized kinetic equations}

\section{Coordinate system}

In this section, the linearized kinetic equations will be transformed to a canonical form, given by Eqs. (5.80) and (5.81). Expressions appear on the right-hand sides of these equations which also appear in the integrands of the expressions for the fluxes. Transport coefficients appear naturally as inner products in this formalism, which was introduced by Bernstein (1974), and the Onsager relations are then just the symmetry properties of the se inner products. The coupling of the kinetic equations, for the two particle species, is also most easily treated within this formalism.

At this point, it is convenient to introduce a poloidal angle coordinate $\theta$, which differs from the coordinate $\Theta$, introduced in Sec. II.C. Many formulae are simplified by choosing $\theta$ such that

$$
\overrightarrow{\mathrm{B}} \cdot \nabla \theta=\overrightarrow{\mathrm{B}}_{p} \cdot \nabla \theta=k,
$$


where $k$ is independent of $\theta$ (and $\zeta$ ). This means that $\theta$ may be obtained from a knowledge of the poloidal field

$$
\theta=k \int_{0}^{l_{p}} \frac{d l_{p}}{B_{p}}
$$

where the integration path is tangent to the poloidal projection of a field line (on a plane of constant $\zeta$ ). The requirement that $\theta$ increase by $2 \pi$ on one poloidal circuit around the magnetic axis implies that

$$
k=2 \pi / \oint \frac{d l_{p}}{B_{p}} \text {. }
$$

Note that $k$ is also related to the derivative with respect to poloidal flux of the volume enclosed by a flux surface

$$
\frac{1}{2 \pi} \frac{d V}{d \psi}=\oint \frac{d \theta}{\overrightarrow{\mathrm{B}} \cdot \nabla \theta}=\oint \frac{d l_{p}}{B_{p}}=\frac{2 \pi}{k}
$$

and to the metric determinant of Eq. (2.43): $k=g^{-1 / 2}$.

The natural choice of $\zeta$ as the symmetry angle, in axisymmetric systems, requires that $\overrightarrow{\mathrm{B}} \cdot \nabla \zeta=B_{T} / R$ which is not constant. Therefore, the local pitch of the field lines, $\overrightarrow{\mathrm{B}} \cdot \nabla \zeta / \overrightarrow{\mathrm{B}} \cdot \nabla \theta$, is not constant, i.e., the field lines are not "straight," in these coordinates, in contrast to those used in Eq. (2.49).

The flux surface average, defined by Eq. (2.56), may be written as

$$
\langle A\rangle=\oint \frac{d l_{p}}{B_{p}} A / \oint \frac{d l_{p}}{B_{p}} .
$$

With the above choice of poloidal angle $\theta$, this is simply

$$
\langle A\rangle=\oint \frac{d \theta}{2 \pi} A \text {. }
$$

In place of one of the flux coordinates $\psi, \phi$, or $V$, as a label for the flux surfaces, we shall use a coordinate $\rho$ which has the dimensions of length. This will make the results easier to understand, since the dimensions of various quantities (e.g., diffusion coefficients) will be the physical ones. We define the effective minor radius coordinate $\rho$ in terms of the toroidal flux $\phi$ by the relation

$$
\phi=B_{\mathbf{T o}} \cdot \pi \rho^{2},
$$

where $B_{\mathrm{Tb}}$ is a constant, representative value of the toroidal field; in general, $\rho$ is then a typical dimension of the magnetic surface cross section. It reduces to the usual minor radius coordinate in the large aspect ratio, circular cross section case, as discussed in Sec. VI. The advantage of this particular definition, in terms of toroidal flux, will become apparent in Sec. VII.

We also define an effective poloidal field magnitude in terms of the minor radius coordinate $\rho$, by

$$
B_{\mathrm{po}}(\rho, t)=\frac{1}{R_{0}} \frac{\partial \psi}{\partial \rho},
$$

so that the poloidal field vector is

$$
\overrightarrow{\mathrm{B}}_{p}=\left(\hat{e}_{\zeta} \times \nabla \rho\right)\left(R_{\mathrm{o}} / R\right) B_{\mathrm{po}}(\rho, t) .
$$

Here $R_{0}$ is an arbitrarily chosen constant, a typical major radius, and $\hat{e}_{\zeta}$ is a unit vector in the toroidal direction. The drift velocity component which is needed is $\overrightarrow{\mathrm{v}}_{d} \cdot \nabla \rho=\overrightarrow{\mathrm{v}}_{d} \cdot \nabla \psi /(\partial \psi / \partial \rho)$, which can be written as

$$
\overrightarrow{\mathrm{v}}_{\boldsymbol{d}} \cdot \nabla \rho=\left(B_{\mathrm{To}} / B_{\mathrm{po}}\right) v_{\|} \hat{n} \cdot \nabla\left(v_{\|} / \Omega\right),
$$

where we have used $R B_{T}=R_{0} B_{\mathrm{Tb}}$. Further,' we write

$$
B=B_{0} / h,
$$

so that

$$
\overrightarrow{\mathrm{v}}_{d} \cdot \nabla \rho=\left(v_{\|} / \Omega_{p}\right) \hat{n} \cdot \nabla\left(v_{\|} h\right),
$$

where

$$
\Omega_{\mathrm{p}} \equiv\left(e B_{0} / m c\right)\left(B_{\mathrm{po}} / B_{\mathrm{To}}\right) \simeq e B_{\mathrm{po}} / m c
$$

is a typical value for the gyrofrequency in the poloidal magnetic field. We shall usually use the approximation $B \simeq B_{T}$, so that $h \simeq R / R_{0}$.

\section{Transformation of the kinetic equations}

Rather than work with Eqs. (5.21) and (5.29), it is useful to transform them by introducing the functions $H_{e}, H_{i}$, defined as follows (now using $-e$ for the electron charge and $Z_{i} e$ for the ion charge)

$$
\begin{aligned}
f_{e 1}= & \frac{2 v_{\|} u_{i \|}}{v_{\text {the }}^{2}} f_{e 0}-\left(e / T_{e}\right) f_{e 0} \int_{0}^{l_{p}} \frac{d l_{p}}{B_{p}}\left[B E_{\|}-B^{2}\left\langle E_{\|} B\right\rangle /\left\langle B^{2}\right\rangle\right] \\
& +v_{\|} f_{s e} B\left\langle E_{\|} B\right\rangle /\left\langle B^{2}\right\rangle+H_{e}, \\
f_{i 1}= & -\frac{v_{\|} h}{\Omega_{i p}}\left[\frac{\partial \ln p_{i}}{\partial \rho}+\frac{Z_{i} e}{T_{i}} \frac{\partial\langle\Phi\rangle}{\partial \rho}\right] f_{i 0} \\
& +\left(Z_{i} e / T_{i}\right) f_{i 0} \int_{0}^{l_{p}} \frac{d l_{p}}{B_{p}}\left[B E_{*}-B^{2}\left\langle E_{*} B\right\rangle /\left\langle B^{2}\right\rangle\right]+H_{i},
\end{aligned}
$$

where $E_{*} \equiv E_{\|}+F_{\| i} / n_{i 0} Z_{i} e$ is the "effective electric field" acting on the ions, allowing for friction with the electrons. The purpose of introducing the first term in Eq. (5.42) is to cancel the term proportional to $u_{i \|}$, contained in $C_{e i}^{l}$, in Eq. (5.21); recall Eqs. (4.41)-(4.46).

In the second term in Eq. (5.42), we have introduced the integral of $E_{\|}$along a field line, and subtracted a term proportional to the average $\left\langle E_{\|} B\right\rangle$, to make the integral single valued in $\theta$. The purpose of these terms is to eliminate the $\theta$ dependence of $E_{\|}$from the kinetic equation, so that only the average $\left\langle E_{\|} B\right\rangle$ appears; note that the electrostatic field makes no contribution to this average, from Eq. (2.53). The third term in Eq. (5.42) involves the Spitzer function, $f_{\text {se }}$, defined in Sec. IV

$$
C_{e}\left(v_{\|} f_{s e}\right) \equiv\left(C_{e e}+\nu_{e i} \&\right) v_{\|} f_{s e}=\left(e / T_{e}\right) v_{\|} f_{e 0} .
$$

This is conventional, following Rosenbluth, Hazeltine and Hinton (1972), and makes possible the exact solution for $H_{e}$ in the limit of a small fraction of trapped particles [see Sec. VI.B]. In Eq. (5.44) and below, we suppress the superscript $l$ on the linearized collision operators; the subscript zero, identifying the homogeneous electron collision operator, is also omitted.

The introduction of the ion pressure gradient and "radial" electric field, in Eq. (5.43), eliminates these from Eq. (5.29). The purpose of the other terms is to eliminate the $\theta$ dependence of $E_{\|}$and $F_{\|_{i}}$.

Thus the function $H_{e}$ satisfies the following equation 


$$
\begin{aligned}
\overrightarrow{\mathrm{v}}_{\|} \circ \nabla H_{e}-C_{e} H_{e}=- & \overrightarrow{\mathrm{v}}_{d e} \cdot \nabla \rho\left[\frac{\partial}{\partial \rho} \ln p_{e}+\left(T_{i} / Z_{i} T_{e}\right) \frac{\partial \ln p_{i}}{\partial \rho}\right. \\
& \left.+\left(v^{2} / v_{\mathrm{the}}^{2}-5 / 2\right) \frac{\partial \ln T_{e}}{\partial \rho}\right] f_{e 0} \\
& -\overrightarrow{\mathrm{v}}_{\|} \cdot \nabla\left(v_{\|} / h\right) f_{s e} B_{0}\left\langle E_{\|} B\right\rangle /\left\langle B^{2}\right\rangle \\
& -\mu_{i}\left(T_{i} / Z_{i} T_{e}\right)\left(v_{\|} /\left|\Omega_{e p}\right|\right) \hat{n} \cdot \nabla\left(v_{\|} / h\right) f_{e 0},
\end{aligned}
$$

where $\mu_{i}$ is related to $u_{i \|}$ by

$$
u_{i \|}=\left(v_{\text {thi }}^{2} / 2 \Omega_{i p}\right)\left\{\mu_{i} / h-\left[\frac{\partial \ln p_{i}}{\partial \rho}+\left(Z_{i} e / T_{i}\right) \frac{\partial\langle\Phi\rangle}{\partial \rho}\right] h\right\},
$$

which follows from Eq. (5.43) and the definition of $u_{i \|}$, with

$$
\int d^{3} v v_{\|} H_{i} \equiv\left(n_{i 0} v_{t h i}^{2} / 2 \Omega_{i p}\right) \mu_{i} / h .
$$

The fact that this parallel ion flow is proportional to $B$ $\left(=B_{0} / h\right)$ follows from the fact that it must be divergenceless, since the other terms in Eq. (5.46) provide the parallel return flows discussed in Sec. $\Pi$. Note that the radial electric field $-\partial\langle\Phi\rangle / \partial \rho$ has cancelled and does not appear in Eq. (5.45)。

The equation satisfied by the function $H_{i}$ is

$$
\begin{aligned}
\overrightarrow{\mathrm{v}}_{\|} \cdot \nabla H_{i}-C_{i i} H_{i}= & -\overrightarrow{\mathrm{v}}_{d i} \cdot \nabla \rho\left(v^{2} / v_{\mathrm{th} i}^{2}-5 / 2\right) \frac{\partial \ln T_{i}}{\partial \rho} f_{i 0} \\
& +\left(Z_{i} e / T_{i}\right) \frac{B_{0}\left\langle E_{*} B\right\rangle}{\left\langle B^{2}\right\rangle} \frac{v_{\|}}{h} f_{i 0} .
\end{aligned}
$$

The term $\left\langle E_{*} B\right\rangle$, which contains the friction force, can be expressed as an integral of $H_{e}$, as follows. By multiplying Eq. (5.21) by $m_{e} v_{\|}$and integrating over all velocities, we obtain the parallel electron momentum equation:

$$
\int d^{3} v m_{e} v_{\|} \overrightarrow{\mathrm{v}}_{\|} \cdot \nabla f_{e 1}=F_{\| e}-n_{e 0} e E_{\|} 。
$$

We now multiply this equation by $B$ and average over a magnetic surface, integrating the left-hand side by parts, to obtain [compare Eq. (2.96)]

$$
\begin{aligned}
-m_{e} B_{0}\left\langle\int d^{3} v f_{e 1} \overrightarrow{\mathrm{v}}_{\|} \circ \nabla\left(v_{\|} / h\right)\right\rangle & =\left\langle\left(F_{\| e}-n_{e 0} e E_{\|}\right) B\right\rangle \\
& =-n_{e 0} e\left\langle E_{*} B\right\rangle,
\end{aligned}
$$

where the second equality follows from charge neutrality $n_{e 0}=Z_{i} n_{i 0}$. When Eq. $(5.42)$ is substituted into Eq. (5.49), one finds that $f_{e 1}$, on the left-hand side, may be replaced by $H_{e}$. Thus

$$
\left\langle\int d^{3} v H_{e^{\vec{v}_{\|}}} \circ \nabla\left(v_{\|} / h\right)\right\rangle=\left(n_{e 0} e / m_{e}\right)\left\langle E_{*} B\right\rangle / B_{0} .
$$

After identification of the four "thermodynamic forces" $A_{n e}$, and the two forces $A_{n i}$, defined below, the equations for $H_{e}$ and $H_{i}$ may be written in the following useful form

$$
\begin{aligned}
& \overrightarrow{\mathrm{v}}_{\|} \cdot \nabla H_{e}-C_{e} H_{e}=-\sum_{n=1}^{4} \alpha_{n} A_{n e} f_{e 0}, \\
& \overrightarrow{\mathrm{v}}_{\|} \cdot \nabla H_{i}-C_{i i} H_{i}=-\sum_{n=1}^{2} \beta_{n} A_{n i} f_{i 0},
\end{aligned}
$$

where

$$
\begin{aligned}
& \alpha_{1}=\overrightarrow{\mathrm{v}}_{d e} \cdot \nabla \rho, \quad \alpha_{2}=\left(v^{2} / v_{\text {the }}^{2}-5 / 2\right) \overrightarrow{\mathrm{v}}_{d e} \cdot \nabla \rho \\
& \alpha_{3}=\overrightarrow{\mathrm{v}}_{\|} \cdot \nabla\left(v_{\|} / h\right) f_{s e} / f_{e 0}, \quad \alpha_{4}=-\left(v_{\|} / \mid \Omega_{e p}\right) \hat{n} \cdot \nabla\left(v_{\|} / h\right) \\
& \left.\beta_{1}=\left(2 \Omega_{i p} / n_{i 0} v_{\mathrm{th} i}^{2}\left\langle h^{-2}\right\rangle\right) v_{\|} / h, \quad \beta_{2}=\left(v^{2} / v_{\text {thi }}^{2}-5 / 2\right) \overrightarrow{\mathrm{v}}_{d i} \cdot \nabla \rho .54\right)
\end{aligned}
$$

The forces have been chosen as follows

$$
\begin{aligned}
& A_{1 e}=\frac{\partial}{\partial \rho} \ln p_{e}+\left(T_{i} / Z_{i} T_{e}\right) \frac{\partial}{\partial \rho} \ln p_{i}, \\
& A_{2 e}=\frac{\partial}{\partial \rho} \ln T_{e}, \quad A_{3 e}=B_{0}\left\langle E_{\|} B\right\rangle /\left\langle B^{2}\right\rangle, \\
& A_{4 e}=-\left(T_{i} / Z_{i} T_{e}\right) \mu_{i}, \\
& A_{1 i}=-\left(n_{i 0} Z_{i} e / m_{i} \Omega_{i p}\right)\left\langle E_{*} B\right\rangle / B_{0}, A_{2 i}=\frac{\partial}{\partial \rho} \ln T_{i} .
\end{aligned}
$$

The driving term $A_{1 i}$ has been expressed as an integral of $H_{e}$ in Eq. (5.50); this equation can also be written as

$$
A_{1 i}=\left(1 / Z_{i}\right)\left\langle\int d^{3} v H_{e} \alpha_{4}\right\rangle .
$$

Similarly, by using Eq. (5.47), the driving term $A_{4 e}$ can be expressed as an averaged moment of $H_{i}$

$$
A_{4 e}=-\left(T_{i} / Z_{i} T_{e}\right)\left\langle\int d^{3} v H_{i} \beta_{i}\right\rangle .
$$

From the linearity of Eqs. (5.51) and (5.52), it follows that the right-hand sides of Eqs. (5.60) and (5.61) may be written as linear combinations of the six forces $A_{n e}, A_{n i}$. Then $A_{1 i}$ and $A_{4 e}$ may be eliminated, by expressing them in terms of the four independent forces $A_{1 e}, A_{2 e}, A_{3 e}$, and $A_{2 i}$. Before doing this, we introduce the transport coefficients.

\section{Transport coefficients}

The fluxes, or moments of the distribution function, which are needed to close the set of moment equations [see Sec. VII for a more detailed discussion] are the electron flux $\Gamma_{e}$, the electron heat flux $q_{e}$, the ion heat flux $q_{i}$, and an average of the parallel current density $J_{\|}$:

$$
\begin{aligned}
& \Gamma_{e} \equiv\left\langle\int d^{3} v \overrightarrow{\mathrm{v}}_{d e} \cdot \nabla \rho H_{e}\right\rangle=\left\langle\int d^{3} v \alpha_{1} H_{e}\right\rangle, \\
& q_{e} / T_{e} \equiv\left\langle\int d^{3} v \overrightarrow{\mathrm{v}}_{d e} \cdot \nabla \rho\left(v^{2} / v_{\mathrm{the}}^{2}-5 / 2\right) H_{e}\right\rangle=\left\langle\int d^{3} v \alpha_{2} \boldsymbol{H}_{e}\right\rangle, \\
& q_{i} / T_{i} \equiv\left\langle\int d^{3} v \overrightarrow{\mathrm{v}}_{d i} \cdot \nabla \rho\left(v^{2} / v_{\mathrm{thi}}^{2}-5 / 2\right) H_{i}\right\rangle=\left\langle\int d^{3} v \beta_{2} H_{i}\right\rangle, \\
& \left\langle\left(J_{\|}-J_{\| s}\right) / h\right\rangle \equiv-e\left\langle\int d^{3} v H_{e} v_{\|} / h\right\rangle .
\end{aligned}
$$

Here we have introduced the Spitzer current density, defined for present purposes as 


$$
J_{\| s} \equiv \sigma_{\|} B\left\langle E_{\|} B\right\rangle /\left\langle B^{2}\right\rangle,
$$

where $\sigma_{\|}$is given in Table II of Sec. IV. An approximate analytical expression for the $Z_{i}$ dependence is

$$
\sigma_{\|}=\frac{n_{e} e^{2} \tau_{e}}{m_{e^{v}}}\left[0.29+\frac{0.46}{1.08+Z_{i}}\right]^{-1} \text {. }
$$

We now show that Eq. (5.65) can be written in terms of an integral of $\alpha_{3}$. By using the definition of $\alpha_{3}$ and integrating by parts in the flux surface average, we have

$$
\left\langle\int d^{3} v \alpha_{3} H_{e}\right\rangle=-\left\langle\int d^{3} v\left(v_{\|} / h\right)\left(f_{s e} / f_{e 0}\right) \overrightarrow{\mathrm{v}}_{\|} \cdot \nabla H_{e}\right\rangle,
$$

where $\overrightarrow{\mathrm{v}}_{\|} \circ \nabla H_{e}$ may be replaced by $C_{e} H_{e}$, using Eq. (5.51). Then the self-adjointness property of the linearized collision operator $C_{e} \equiv C_{e e}+\nu_{e i} \mathcal{L}$, which is expressed by [recall Eq. (4.63)]

$$
\int d^{3} v\left(g_{1} / f_{e 0}\right) C_{e} f_{1}=\int d^{3} v\left(f_{1} / f_{e 0}\right) C_{e} g_{1},
$$

enables us to write

$$
\left\langle\int d^{3} v \alpha_{3} H_{e}\right\rangle=-\left\langle\frac{1}{h} \int d^{3} v\left(H_{e} / f_{e 0}\right) C_{e}\left(v_{\|} f_{s e}\right)\right\rangle .
$$

From Eq. (5.44), the definition of $f_{s e}$, we have

$$
\left\langle\int d^{3} v \alpha_{3} H_{e}\right\rangle=-\left(e / T_{e}\right)\left\langle\frac{1}{h} \int d^{3} v v_{\|} H_{e}\right\rangle,
$$

therefore, from Eq. (5.65),

$$
\frac{1}{T_{e}}\left\langle\left(J_{\|}-J_{\| s}\right) / h\right\rangle=\left\langle\int d^{3} v \alpha_{3} H_{e}\right\rangle .
$$

Eqs. (5.62), (5.63), (5.64), and (5.68) thus give the required fluxes in terms of simple integrals of $H_{e}$ and $H_{i}$, in which the functions $\alpha_{1}, \alpha_{2}, \alpha_{3}, \beta_{2}$ appear. These are the same functions which multiply the forces $A_{n e}, A_{n i}$ on the right-hand sides of Eqs. (5.51) and (5.52), and this makes our choice of fluxes and forces consistent with Onsager symmetry of the transport coefficients [see Sec. V.C].

From the linearity of Eqs. (5.51) and (5.52), we may write their solutions in the form

$$
H_{e}=\sum_{n=1}^{4} g_{n e} A_{n e}, \quad H_{i}=\sum_{n=1}^{2} g_{n i} A_{n i} .
$$

The required fluxes may then be written as linear combinations of all of the $A_{n}$ 's

$$
\begin{aligned}
& \Gamma_{e}=\sum_{n=1}^{4}\left(\alpha_{1}, g_{n e}\right) A_{n e}, \\
& q_{e} / T_{e}=\sum_{n=1}^{4}\left(\alpha_{2}, g_{n e}\right) A_{n e}, \\
& \left(1 / T_{e}\right)\left\langle\left(J_{\|}-J_{\| s}\right) / h\right\rangle=\sum_{n=1}^{4}\left(\alpha_{3}, g_{n e}\right) A_{n e}, \\
& q_{i} / T_{i}=\sum_{n=1}^{2}\left(\beta_{2}, g_{n i}\right) A_{n i} .
\end{aligned}
$$

Here, following Bernstein (1974), we have introduced inner products, defined, for example, by

$$
\left(\alpha_{1}, g_{n e}\right) \equiv\left\langle\int d^{3} v \alpha_{1} g_{n e}\right\rangle \text {. }
$$

These inner products are the transport coefficients, relating the fluxes to the forces.

We now eliminate the "forces" $A_{1 i}$ and $A_{4 e}$, which also appears as fluxes, according to Eqs. (5.60) and (5.61); by substituting Eq. (5.69) into these equations, we find

$$
\begin{aligned}
& A_{1 i}=\left(1 / Z_{i}\right) \sum_{n=1}^{4}\left(\alpha_{4}, g_{n e}\right) A_{n e}, \\
& A_{4 e}=-\left(T_{i} / Z_{i} T_{e}\right) \sum_{n=1}^{2}\left(\beta_{1}, g_{n i}\right) A_{n i} .
\end{aligned}
$$

These equations may be solved for $A_{1 i}$ and $A_{4 e}$, in terms of the other $A_{n}$ 's, to give

$$
\begin{gathered}
A_{1 i}=\left(1 / Z_{i} F\right)\left\{\sum_{n=1}^{3}\left(\alpha_{4}, g_{n e}\right) A_{n e}\right. \\
\left.-\left(T_{i} / Z_{i} T_{e}\right)\left(\alpha_{4}, g_{4 e}\right)\left(\beta_{1}, g_{2 i}\right) A_{2 i}\right\}, \\
A_{4 e}=-\left(T_{i} / Z_{i} T_{e} F\right)\left\{\left(1 / Z_{i}\right)\left(\beta_{1}, g_{1 i}\right) \sum_{n=1}^{3}\left(\alpha_{4}, g_{n e}\right) A_{n e}\right. \\
\left.+\left(\beta_{1}, g_{2 i}\right) A_{2 i}\right\},
\end{gathered}
$$

where

$$
F \equiv 1+\left(T_{i} / Z_{i}^{2} T_{e}\right)\left(\alpha_{4}, g_{4 e}\right)\left(\beta_{1}, g_{1 i}\right) .
$$

When Eqs. (5.77) and (5.78) are substituted into Eqs. (5.70)-(5.73), we finally have the four fluxes expressed in terms of the four independent forces $A_{1 e}, A_{2 e}, A_{3 e}$, and $A_{2 i}$. We shall show in Sec. V.C that both inner products which appear in Eq. (5.79) are negative, so that $F \geqslant 1$.

The transport problem has been reduced to solving for the functions $g_{n e}, g_{n i}$ and calculating the transport coefficients in the form of inner products, $\left(\alpha_{m}, g_{n e}\right),\left(\beta_{m}, g_{n i}\right)$. The equations to be solved are obtained by substituting Eq. (5.69) into Eqs. (5.51) and (5.52)

$$
\begin{aligned}
& \overrightarrow{\mathrm{v}}_{\| 1} \cdot \nabla g_{n e}-C_{e} g_{n e}=-\alpha_{n} f_{e 0}, \quad n=1,2,3,4, \\
& \overrightarrow{\mathrm{v}}_{\| 1} \cdot \nabla g_{n i}-C_{i i} g_{n i}=-\beta_{n} f_{i 0}, \quad n=1,2 .
\end{aligned}
$$

These equations may be solved variationally, as discussed in Subsection V.C. In the large aspect-ratio limiting case, explicit analytical solutions may be obtained, as discussed in Sec. VI.

\section{Weak-coupling approximation}

The above formalism is simplified considerably by an approximation which we shall call the "weak-coupling approximation." The effect on the ions of collisions with the electrons is contained in the "force" $A_{1 i}$ in Eq. (5.52); this equation becomes decoupled from the equation for $H_{e}$ if we neglect $A_{1 i}$. If this is done, Eq. (5.73) gives

$$
q_{i} / T_{i} \simeq\left(\beta_{2}, g_{2 i}\right) A_{2 i},
$$

while Eq. (5.76) yields

$$
A_{4 e} \equiv-\left(T_{i} / Z_{i} T_{e}\right) \mu_{i} \simeq-\left(T_{i} / Z_{i} T_{e}\right)\left(\beta_{1}, g_{2 i}\right) A_{2 i},
$$

where $A_{2 i} \equiv \partial\left(\ln T_{i}\right) / \partial \rho$. These are the lowest-order results, in which the ion fluxes are unaffected by the electron forces, and the ion force $A_{2 i}$ appears in the expressions for the electron fluxes in a simple additive way, 
through $A_{4 e}$.

A first correction to Eq. (5.82) may be obtained by substituting Eq. (5.83) into Eq. (5.75); we obtain

$$
q_{i}-T_{i}\left(\beta_{2}, g_{2 i}\right) A_{2 i}=T_{i}\left(\beta_{2}, g_{1 i}\right) A_{1 i},
$$

with

$$
\begin{aligned}
A_{1 i} \simeq\left(1 / Z_{i}\right) & \left\{\sum_{n=1}^{3}\left(\alpha_{4}, g_{n e}\right) A_{n e}\right. \\
& \left.-\left(T_{i} / Z_{i} T_{e}\right)\left(\alpha_{4}, g_{4 e}\right)\left(\beta_{1}, g_{2 i}\right) A_{2 i}\right\} .
\end{aligned}
$$

By comparison with Eq. (5.77), we see that the weakcoupling approximation is equivalent to assuming $F \simeq 1$, or, more precisely,

$$
F-1 \equiv\left(T_{i} / Z_{i}^{2} T_{e}\right)\left(\alpha_{4}, g_{4 e}\right)\left(\beta_{1}, g_{1 i}\right) \ll 1 .
$$

More specific conditions will be given in Sec. VI, for the various collisionality regimes, after the transport coefficients have been obtained for the large aspect ratio case. We will find that the weak-coupling approximation is satisfied, in the electron-ion problem, under most conditions.

\section{Two ion-species problem}

As a first step in the solution of the problem of transport in the presence of impurities, we consider the special case of one-impurity species. We consider a restricted problem, in which the two ion species have disparate masses. In this case, we may simply transpose the results, for the inner products, from the electron-ion problem to the two ion species problem.

Let the two ion species be labeled " $a$ " and " $b$," and assume $m_{a} \ll m_{b}$. With the ordering $m_{a} / m_{b} \sim \delta_{p a} \sim \delta_{b b}^{2}$, the fastest process is temperature equilibration, so that we must assume $T_{a} \simeq T_{b}$. Diffusion due to unlike-species collisions occurs on a faster time scale than that due to like-species collisions, so only the first-order kinetic equations are needed. The analys is of the electron-ion problem applies, with $m_{e}$ replaced by $m_{a}, m_{i}$ by $m_{b},-e$ by $Z_{a} e$, etc.

The effect of collisions with the electrons is of higherorder, but could be included by replacing $E_{\|}$by $E_{\|}-F_{\|}^{e c} /$ $n_{c} Z_{c} e$, where $c$ denotes either $a$ or $b$, and $F_{\|}^{e c}$ is the friction of that ion species with the electrons. However, this would necessitate solution of the electron kinetic equation in the presence of two ion species, and would complicate the results. Since the effect of these terms on the particle and heat fluxes can be shown to be relatively small, we shall replace $E_{\|}$by zero. By Onsager symmetry, we thereby also neglect the contribution to the "bootstrap current" (see Sec.V.D) from friction between the two ion species. Although this contribution is not negligible (Connor, 1973), when one or both of the ion species is in the banana regime, reliable results for the numerical coefficients are not yet available.

We therefore consider the three fluxes $\Gamma_{a}$ (the species " $a$ " particle flux), $q_{a}$, and $q_{b}$ (the heat fluxes), and the three forces

$$
A_{1 a}=\frac{\partial}{\partial \rho} \ln p_{a}-\left(Z_{a} T_{b} / Z_{b} T_{a}\right) \frac{\partial}{\partial \rho} \ln p_{b} .
$$

$$
A_{2 a}=\frac{\partial}{\partial \rho} \ln T_{a}, \quad A_{2 b}=\frac{\partial}{\partial \rho} \ln T_{b} .
$$

Neglecting the effect of collisions with the electrons, the species " $b$ " particle flux is given by the ambipolar condition

$$
Z_{a} \Gamma_{a}+Z_{b} \Gamma_{b} \simeq 0
$$

The relations between the fluxes and forces are very similar to Eq. (5.70)-(5.73)

$$
\begin{aligned}
& \Gamma_{a}=\sum_{n=1}^{3}\left(\alpha_{1}, g_{n a}\right) A_{n a}, \\
& q_{a} / T_{a}=\sum_{n=1}^{3}\left(\alpha_{2}, g_{n a}\right) A_{n a}, \\
& q_{b} / T_{b}=\sum_{n=1}^{2}\left(\beta_{2}, g_{n b}\right) A_{n b},
\end{aligned}
$$

where the auxiliary forces $A_{3 a}$ and $A_{1 b}$, defined by

$$
A_{3 a}=\left(Z_{a} T_{b} / Z_{b} T_{a}\right) \mu_{b}, \quad A_{1 b}=\left\langle F_{\| a} B\right\rangle / m_{b} \Omega_{b p} B_{0}
$$

appear because of the frictional coupling of the two species. [Note that, neglecting the effect of $E_{\|}$, there is no force corresponding to $A_{3 e}$, in Eq. (5.57); the force corresponding to $A_{4 e}$ has therefore been labeled $A_{3 a}$.] These auxiliary forces are eliminated just as in the electronion problem, to give

$$
\begin{gathered}
A_{3 a}=-\left(Z_{a} T_{b} / Z_{b} T_{a} F\right)\left\{\frac{Z_{a}}{Z_{b}}\left(\beta_{1}, g_{1 b}\right) \sum_{n=1}^{2}\left(\alpha_{3}, g_{n a}\right) A_{n a}\right. \\
\left.-\left(\beta_{1}, g_{2 b}\right) A_{2 b}\right\}, \\
A_{1 b}=-\left(Z_{a} / Z_{b} F\right)\left\{\sum_{n=1}^{2}\left(\alpha_{3}, g_{n a}\right) A_{n a}\right. \\
\left.+\left(Z_{a} T_{b} / Z_{b} T_{a}\right)\left(\alpha_{3}, g_{3 a}\right)\left(\beta_{1}, g_{2 b}\right) A_{2 b}\right\},
\end{gathered}
$$

where

$$
F \equiv 1+\left(Z_{a}^{2} T_{b} / Z_{b}^{2} T_{a}\right)\left(\alpha_{2}, g_{3 a}\right)\left(\beta_{1}, g_{1 b}\right) \geqslant 1 .
$$

There is a major difference between the problem of two ion species, and the electron-ion problem. In the former problem, the weak-coupling approximation is not generally valid, for the parameter ranges relevant to tokamak experiments, while in the latter problem, it is generally valid. This question will be pursued further in Sec. VI, after specific results for the transport coefficients have been obtained.

\section{General variational principle}

The basis of the variational method for solving the kinetic equations, Eqs. (5.80) and (5.81), is the principle of minimum entropy production (Prigogine, 1961). In order to illustrate the argument which leads to the actual form of the variational principle without undue complication, we consider the ion equation in the weak-coupling approximation: Eq. (5.52) with $A_{1 i} \simeq 0$. The local rate of entropy production due to ion-ion collisions, has the following form, when averaged over a magnetic surface (or, more precisely, over the volume region between two nearby magnetic surfaces) 


$$
\dot{S}_{i}=-\left\langle\int d^{3} v \ln f_{i} C_{i i}\left(f_{i}, f_{i}\right)\right\rangle .
$$

By expanding in the gyroradius, $f_{i} \simeq f_{i 0}+f_{i 1}$, with $f_{i 0}$ Maxwellian and constant on a magnetic surface, we obtain

$$
\dot{S}_{i}=-\left\langle d^{3} v\left(f_{i 1} / f_{i 0}\right) C_{i i} f_{i 1}\right\rangle .
$$

Since the collision term conserves particles and momentum, and has no effect on a shifted Maxwellian, the transformation of Eq. (5.43) leads to

$$
\dot{S}_{i}=-\left\langle d^{3} v \hat{H}_{i} C_{i i} H_{i}\right\rangle \text {, }
$$

where $\hat{H}_{i} \equiv H_{i} / f_{i 0}$ 。

Steady-state ion heat conduction is governed by the law that $\left[\dot{S}_{i}\right]$ is a minimum (where the square brackets indicate the functional of $H_{i}$ ), subject to the constraints imposed by the guiding center drifts and the existence of a nonzero temperature gradient. The subsystem consisting of the plasma between two nearby magnetic surfaces is maintained away from thermal equilibrium, since the drifts and the temperature gradient prevent the distribution function from becoming a Maxwellian. If the subsystem were a closed system, its entropy would increase to the maximum value consistent with the constraints of constant total number of particles and energy, and the distribution function would then be Maxwellian. Actually, the entropy increases indefinitely because a steady state difference is maintained, between the distribution function and the equilibrium Maxwellian. This difference must adjust so that the rate of increase of entropy is as small as possible, however.

The mathematical form of the constraints, imposed by the temperature gradient and the guiding center motion, may be expressed in the form of integrals obtained from Eq. (5.52). We first define the even and odd [in $\sigma$ $\left.\equiv \operatorname{sign}\left(v_{\|}\right)\right]$parts of the distribution function

$$
\begin{aligned}
& H_{i}^{+} \equiv \frac{1}{2}\left[H_{i}(\sigma=+)+H_{i}(\sigma=-)\right], \\
& H_{i}^{-} \equiv \frac{1}{2}\left[H_{i}(\sigma=+)-H_{i}(\sigma=-)\right] .
\end{aligned}
$$

Then Eq. (5.52) yields the following equations for the odd and even parts of $H_{i}$

$$
\begin{aligned}
& \left|v_{\|}\right| \hat{n} \cdot \nabla H_{i}^{-}-C_{i i} H_{i}^{+}=-\overrightarrow{\mathrm{v}}_{d i} \circ \nabla \rho\left(v^{2} / v_{\mathrm{th} i}^{2}-5 / 2\right) \frac{\partial \ln T_{i}}{\partial \rho} f_{i 0}, \\
& \left|v_{\|}\right| \hat{n} \cdot \nabla H_{i}^{+}-C_{i i} H_{i}^{-}=0 .
\end{aligned}
$$

By multiplying Eqs. (5.89) and (5.90) by $\hat{H}_{i}^{+}$and $\hat{H}_{i}^{-}$respectively, and averaging over a magnetic surface, we find the constraints to be

$$
J_{B}-J_{+}-J_{D}=0, J_{B}+J_{-}=0,
$$

where

$$
\begin{aligned}
& J_{B} \equiv\left\langle\int d^{3} v \hat{H}_{i}^{+}\left|v_{\|}\right| \hat{n} \cdot \nabla H_{i}^{-}\right\rangle, \\
& J_{D} \equiv-\left\langle\int d^{3} v \overrightarrow{\mathrm{v}}_{d i} \cdot \nabla \rho\left(v^{2} / v_{\mathrm{th} i}^{2}-5 / 2\right) H_{i}^{+}\right\rangle \frac{\partial \ln T_{i}}{\partial \rho},
\end{aligned}
$$

and

$$
J_{ \pm} \equiv\left\langle\int d^{3} v \hat{H}_{i}^{ \pm} C_{i i} H_{i}^{ \pm}\right\rangle
$$

We note that

$$
J_{+}+J_{-}=-\dot{S}_{i},
$$

so that the entropy production associated with the even and odd parts of $H_{i}$ may be identified as $-J_{+}$and $-J_{-}$, respectively.

The principle of minimum entropy production with the constraints given by Eq. (5.91), may be expressed by the requirement that the functional

$$
\left[J_{+}\right]+\lambda_{+}\left(\left[J_{B}\right]-\left[J_{+}\right]-\left[J_{D}\right]\right)
$$

be stationary with respect to variations in $H_{i}^{+}$, and that

$$
\left[J_{-}\right]+\lambda_{-}\left(\left[J_{B}\right]+\left[J_{-}\right]\right)
$$

be stationary with respect to variations in $H_{i}^{-}$, where $\lambda_{+}$and $\lambda_{-}$are Lagrange multipliers. These variational principles give Euler equations for $H_{i}^{ \pm}$which are consistent with the constraints, provided that $\lambda_{+}=2, \lambda_{-}=-2$. By noticing that only $\left[J_{B}\right]$ contains both $H_{i}^{+}$and $H_{i}^{-}$, we may combine the two variational principles. The functional

$$
\left[\dot{S}_{i}\right] \equiv\left[J_{+}\right]-\left[J_{-}\right]-2\left[J_{B}\right]+2\left[J_{D}\right]
$$

is thus stationary with respect to independent variations of $H_{i}^{+}$and $H_{i}^{-}$. In fact, by using the negative-definite property of the collision term

$$
J_{ \pm} \leqslant 0
$$

(with equality if and only if $H_{i}^{ \pm} \equiv 0$ ), it is easily shown that $\left[\dot{S}_{i}\right]$ is maximal with respect to $H_{i}^{+}$and minimal with respect to $H_{i}^{-}$. Its extremal value is given by $\mathrm{Eq}$. (5.92), or

$$
\dot{S}_{i}=J_{D}=-\left(q_{i} / T_{i}\right) \frac{\partial \ln T_{i}}{\partial \rho},
$$

using Eq. (5.91) and the definition of the heat flux, Eq. (5.64). From Eq. (5.94) we have $\dot{S}_{i}>0$, so that a positive coefficient $\kappa_{i}$ (the ion thermal conductivity), defined by

$$
q_{i}=-\kappa_{i}\left(\partial T_{i} / \partial \rho\right)
$$

may be obtained from the extremal value of the functional $\left[\dot{S}_{i}\right]$ :

$$
\dot{S}_{i}=\kappa_{i}\left(\partial \ln T_{i} / \partial \rho\right)^{2} \text {. }
$$

This variational principle was first introduced by Rutherford (1970) in a form which is somewhat different, but equivalent to Eq. (5.93).

A somewhat more convenient form of the variational principle is obtained when $g_{2 i}$ is used in place of $H_{i}$; the extremal value is then equal to the inner product $-\left(\beta_{2}, g_{2 i}\right)=\kappa_{i}$.

All of the inner products required to obtain all of the fluxes of both particle species may be calculated from the following variational expressions:

$$
\begin{aligned}
{\left[V_{m n}^{(c)}\right]=\left\langle\int d^{3} v[\right.} & \hat{g}_{m c}^{+} C_{c} g_{n c}^{+}-\hat{g}_{m c}^{-} C_{c} g_{n c}^{-} \\
& -\hat{g}_{m c}^{+}\left|v_{\|}\right| \hat{n} \cdot \nabla g_{n c}^{-}-\hat{g}_{n c}^{+}\left|v_{\|}\right| \hat{n} \cdot \nabla g_{m c}^{-} \\
& \left.\left.-g_{m c}^{+} \gamma_{n}^{+}-g_{n c}^{+} \gamma_{m}^{+}+g_{m c}^{-} \gamma_{n}^{-}+g_{n c}^{-} \gamma_{m}^{-}\right]\right\rangle,
\end{aligned}
$$


where $c$ stands for either $e$ or $i, \gamma_{n}$ stands for either $\alpha_{n}$ or $\beta_{n}$, and the superscripts \pm indicate the parity in $\sigma$. [Note that the $\alpha_{n}$ 's are all even, and $\beta_{2}$ is even, but $\beta_{1}$ is odd, so that $\alpha_{n}^{-}=\beta_{2}^{-}=\beta_{1}^{+}=0$.] The errors in these variational expressions are quadratic in the errors in the functions $g_{n c}^{ \pm}$. The diagonal $(m=n)$ terms in the matrix $\left[V_{m n}^{(c)}\right]$ provide mini-max variational principles which are useful in solving for the functions $g_{n e}, g_{n i}$. The extremal values of all of the variational expressions give the required inner products

$$
V_{m n}^{(c)}=-\left\langle\int d^{3} v\left(g_{n c}^{+} \gamma_{m}^{+}-g_{n c}^{-} \gamma_{m}^{-}\right)\right\rangle
$$

which may be verified by substituting Eqs. (5.80) and (5.81), written in terms of the even and odd parts, into Eq. (5.96).

Since all of the $\alpha_{n}$ 's are even, we have, for the electron inner products

$$
V_{m n}^{(e)}=-\left(\alpha_{m}, g_{n e}\right) \text {. }
$$

From the self-adjointness property of $C_{e}$, Eq. (5.67), it follows that Eq. (5.96) is symmetric in $m$ and $n$, and therefore so are the extremal values

$$
\left(\boldsymbol{\alpha}_{m}, g_{n e}\right)=\left(\boldsymbol{\alpha}_{n}, g_{m e}\right) \text {. }
$$

These are the Onsager relations, a consequence of the self-adjointness of the collision operators, which is itself a consequence of microscopic reversibility (de Groot and Mazur, 1962). Furthermore, from the negative-definite property of the collision operators, and the fact that the $\alpha_{m}$ 's are even, it follows that the diagonal matrix elements are negative

$$
\left(\alpha_{n}, g_{n e}\right)=-V_{n n}^{(e)}=\left\langle\int d^{3} v\left[\hat{g}_{n e}^{+} C_{e} g_{n e}^{+}+\hat{g}_{n e}^{-} C_{e} g_{n e}^{-}\right]\right\rangle<0 .
$$

In the ion case, the force $\beta_{2}$ is even, but $\beta_{1}$ is odd. The self-adjointness of $C_{i i}$ implies that $V_{12}^{(i)}=V_{21}^{(i)}$, but Eq. (5.97) then implies antisymmetry of the off-diagonal inner products

$$
\left(\beta_{1}, g_{2 i}\right)=-\left(\beta_{2}, g_{1 i}\right) \text {. }
$$

It should be evident that the diagonal matrix element corresponding to the even force $\beta_{2}$ is negative, as in the electron case

$$
\left(\beta_{2}, g_{2 i}\right)<0 \text {. }
$$

It is easily shown that the other diagonal matrix element is also negative

$$
\left(\beta_{1}, g_{1 i}\right)=V_{11}^{(i)}=\left\langle\int d^{3} v\left[\hat{g}_{1 i}^{+} C_{i i} g_{1 i}^{+}+\hat{g}_{1 i}^{-} C_{i i} g_{1 i}^{-}\right]\right\rangle<0 .
$$

The fact, that all of the diagonal matrix elements are negative, corresponds to the definition of the forces as being positive in the direction opposite to the direction of the fluxes which they drive [e.g., diffusion due to a density gradient goes in the direction opposite to the density gradient]. This is due to the positive-definite nature of the entropy production.

\section{Banana regime}

\section{Definitions}

When the effective collision frequencies of the trapped particles are smaller than their bounce frequencies, the collision term in Eq. (5.80) or (5.81) may be treated formally as a small perturbation. For systems with arbitrary aspect ratio (or an arbitrary fraction of trapped particles) the effective collision frequency for trapped electrons is of the order of the momentum transfer frequency $\nu_{e i}(v)$, defined by Eq. $(5.4)$. The bounce frequency is given roughly by

$$
\omega_{b} \sim\left(2 \pi / L_{c}\right) \cdot v,
$$

where $L_{c}(=2 \pi q R)$ is the connection length. The "banana," or "collisionless" regime for the electrons is then defined by

$$
\nu_{e i} q R / v \ll 1 \text {. }
$$

The banana regime for the ions is defined similarly

$$
\nu_{i i}(v) q R / v \ll 1,
$$

where $\nu_{i i}(v)$ is a 90-degree scattering collision frequency [given, for example by Eq. (6.41)]. Although these conditions are velocity dependent, the assumption is usually made that they can be replaced by the equivalent conditions for particles with the thermal velocity, $v_{\text {the }}$ or $v_{\text {thi }}$. Actually, a more stringent condition is required, in order that Eq. (5.101) hold for all velocities which contribute significantly to the transport processes.

The results which are obtained, for this nearly collisionless regime, are dominated by the guiding-center motion. The diffusion and heat conduction results are consistent with a random walk argument, as given in Sec. III. New effects appear, due to the special properties of these orbits, which are not present in the straight magnetic field case. These are the trapped particle pinch effect and the "bootstrap" current.

\section{Electron distribution function}

We begin the perturbation analysis of Eq. (5.80) by expanding $g_{n e}$ in powers of $\nu_{e i} q R / v(\ll 1)$

$$
g_{n e}=g_{n e}^{(0)}+g_{n e}^{(1)}+\cdots \text {. }
$$

The lowest-order equation, obtained by neglecting the collision term is then

$$
v_{\|} \hat{n} \cdot \nabla g_{n e}^{(0)}=-\alpha_{n} f_{e 0},
$$

where we have treated all of the $\alpha_{n}$ 's uniformly, as being of order unity in the collision frequency parameter. The special property of the $\alpha_{n}$ 's, for the axisymmetric case considered here, is that they have the form $v_{\|} \hat{n} \cdot \nabla(\cdots)$. We shall consider here only the $n=1$ equation in detail; the general solution of Eq. (5.102) is [recall Eq. (3.104)]

$$
g_{1 e}^{(0)}=\left(v_{\|} h /\left|\Omega_{e p}\right|\right) f_{e 0}+G_{1 e},
$$

where

$$
\partial G_{1 e} / \partial \theta=0 \text {. }
$$

The function $G_{1 e}$ is determined by the first-order equation

$$
v_{\| 1} \hat{n} \cdot \nabla g_{1 e}^{(1)}-C_{e} g_{1 e}^{(0)}=0 \text {. }
$$

The unknown function $g_{1 e}^{(1)}$ can be eliminated by the operation $\oint d \theta B / v_{\|}$, where the integration is a round trip between turning points (at which $v_{\text {ll }}$ changes sign) for the trapped particles $\left(h_{\min } \leq \lambda B_{0} \leq h_{\max }\right)$, or a complete circuit around the minor circumference, for the untrapped 
particles $\left(0 \leq \lambda B_{0} \leq h_{\min }\right)$; see Fig. 5 . That this operation annihilates $g_{1 e}^{(1)}$ follows, in the trapped region, from Eq. (3.61); in the untrapped region it follows from requiring that $g_{1 e}^{(1)}$ be single valued. The function $G_{1 e}$ provides the freedom to satisfy these solubility conditions, and is, in turn, determined by them:

$$
\oint \frac{d \theta B}{v_{\mathrm{ll}}} C_{e}\left(\frac{v_{1 \mathrm{ll}} h}{\left|\Omega_{e p}\right|} f_{e 0}+G_{1 e}\right)=0 \text {. }
$$

The perturbation analysis thus leads to a reduction of Eq. (5.80) (for $n=1$ ) to this constraint equation, for a function $G_{1 e}$ which is independent of $\theta$. This analysis was first carried out by Galeev and Sagdeev (1968).

Very similar constraint equations may be obtained for the functions $g_{2 e}, g_{3 e}$, and $g_{4 e}$, by following this same procedure. These constraint equations have not been solved, in general, because of the complexity of the Fokker-Planck collision terms. We will subsequently give an explicit solution for a special case which is tractable: the Lorentz model.

We now show that Eq. (5.106) is equivalent to a variational principle (Rosenbluth, Hazeltine and Hinton, 1972) which is useful in obtaining approximate solutions. We first note that Eq. (5.104) and the boundary conditions imply that $G_{1 e}$ is even in $\sigma$, in the trapped region of phase space, since the odd part of $g_{1 e}^{(0)}$ (and hence of $G_{1 e}$ ) must vanish at the trapped particle bounce points, $\theta=\theta_{1,2}(\lambda)$, and also be independent of $\theta$. Thus,

$$
G_{1 e}(\sigma=+)-G_{1 e}(\sigma=-)=0 \text {, for } h_{\min }<\lambda B_{0}<h_{\max } \text {. }
$$

\section{Variational principle}

The constraint equation, Eq. (5.106), is equivalent to the requirement that the functional

$$
\left[Q_{11}^{(e)}\right] \equiv-\int_{-\pi}^{\pi} \frac{d \theta}{2 \pi} \int d^{3} v\left(\frac{v_{\|} h}{\left|\Omega_{e p}\right|}+\hat{G}_{1 e}\right) C_{e}\left(\frac{v_{\|} h}{\left|\Omega_{e p}\right|} f_{e 0}+G_{1 e}\right)
$$

be a minimum, subject to the constraints of Eqs. (5.104) and (5.107). To prove this, we note that variation of $G_{1 e}$ and use of the self-adjointness property of $C_{e}$, Eq. (5.67), gives

$$
\delta\left[Q_{11}^{(e)}\right]=-\int_{0}^{\infty} v^{3} d v \int_{0}^{h} d \lambda \sum_{\sigma} \int \frac{d \theta B}{\left|v_{\|}\right|} \delta \hat{G}_{1 e} C_{e}\left(\frac{v_{11} h}{\left|\Omega_{e p}\right|} f_{e 0}+G_{1 e}\right),
$$

where we have used

$$
\int d^{3} v=2 \pi \int_{0}^{\infty} v^{3} d v \int_{0}^{h} \frac{B d \lambda}{2\left|v_{\|}\right|} \sum_{\sigma},
$$

and interchanged the order of the $\lambda$ and $\theta$ integrations. The $\theta$ limits of integration are the turning points $\theta_{1,2}(\lambda)$ for the trapped particles, and $\mp \pi$ for the untrapped particles. By using the constraints of Eqs. (5.104) and $(5.107)$ as applied to $\delta \hat{G}_{1 e}$, Eq. (5.109) implies

$$
\int_{-\pi}^{\pi} \frac{d \theta B}{\left|v_{\|}\right|} C_{e}\left(\frac{v_{\|} h}{\left|\Omega_{e p}\right|} f_{e 0}+G_{1 e}\right)=0
$$

for $0 \leq \lambda B_{0} \leq h_{\min }$ (untrapped) which holds for $\sigma=+$ and $\sigma=-$ separately, and

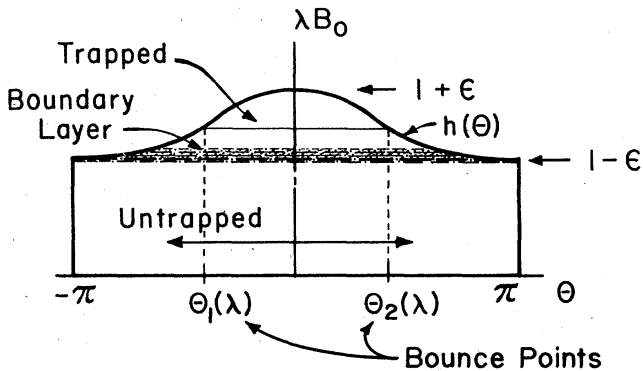

FIG. 5. Phase space, showing trapped and untrapped regions, the boundary layer, and the trapped-particle bounce points. The values $1+\epsilon$ and $1-\epsilon$ for $h_{\max }$ and $h_{\min }$ apply only to the large aspect-ratio, circular cross section case, where $\epsilon=r / R_{0}$.

$$
\sum_{\sigma} \int_{\theta_{1}(\lambda)}^{\theta_{2}(\lambda)} \frac{d \theta B}{\left|v_{\|}\right|} C_{e}\left(\frac{v_{\|} h}{\left|\Omega_{e p}\right|} f_{e 0}+G_{1 e}\right)=0,
$$

for $h_{\min } \leq \lambda B_{0} \leq h_{\max }$ (trapped). Since these equations are equivalent to Eq. (5.106), the variational property of $\left[Q_{11}^{(e)}\right]$ has been demonstrated. That $\left[Q_{11}^{(e)}\right]$ has a minimum follows from the negative-definite property of the collision operator.

A general property of $G_{1 e}$ follows from Eqs. (5.111) and (5.110) (when summed over $\sigma$ ) and the fact that the collision term preserves the parity in $\sigma$ [e.g., $C_{e}\left(v_{\mathrm{II}} f_{e 0}\right)$ is odd in $\sigma]$ :

$$
G_{1 e}(\sigma=+)+G_{1 e}(\sigma=-)=0 \text { for all } \lambda,
$$

i.e., the function $G_{1 e}$ is odd in $\sigma$. It follows from Eq. $(5.107)$ that $G_{1 e}$ is identically zero in the trapped particle region.

The variational expression for $\left[Q_{11}^{(e)}\right]$ may also be obtained directly from Eq. (5.96). We note that $g_{1 e}^{+}$is first order in $\nu_{e i} q R / v$, and that the trial functions must satisfy the constraints of Eqs. (5.103) and (5.104), which imply the cancellation of $\hat{g}_{1 e}^{+}\left|v_{\|}\right| \hat{n} \cdot \nabla g_{n e}^{-}$and $g_{1 e}^{+} \alpha_{1}^{+}$, to first order. Therefore, since $\alpha_{1}^{-}=0$, we have

$$
\left[Q_{11}^{(e)}\right] \simeq\left[V_{11}^{(e)}\right]
$$

neglecting terms quadratic in $\nu_{e i} q R / v$. Furthermore, from Eq. (5.97), it follows that the extremal value is the required transport coefficient, in this case the diffusion coefficient

$$
Q_{11}^{(e)}=-\left(\alpha_{1}, g_{1 e}\right) \text {. }
$$

The other transport coefficients may be obtained from variational principles also:

$$
\left[Q_{m n}^{(e)}\right]=-\left\langle\int d^{3} v \hat{g}_{m e}^{(0)} C_{e} g_{n e}^{(0)}\right\rangle,
$$

where $g_{n e}^{(0)}$ is a solution of Eq. (5.102). By using

$$
\alpha_{n} \equiv v_{\|} \hat{n} \cdot \nabla\left(v_{\|} \gamma_{n}\right)
$$

to define the functions $\gamma_{n}$, i.e., $\gamma_{1}=-h /\left|\Omega_{e p}\right|$ $\gamma_{2}=\left(v^{2} / v_{\text {the }}^{2}-5 / 2\right) \gamma_{1}, \gamma_{3}=\hat{f}_{s e} / h$, and $\gamma_{4}=-1 / h\left|\Omega_{e p}\right|$, the solution of Eq. (5.102) is seen to be

$$
g_{n e}^{(0)}=-v_{\| 1} \gamma_{n} f_{e 0}+G_{n e},
$$

where $\partial G_{n e} / \partial \theta=0$. The extremal values of these func- 
tionals are the transport coefficients:

$$
Q_{m n}^{(e)}=-\left(\alpha_{m}, g_{n e}\right) \text {. }
$$

\section{Lorentz model}

We now consider the special case $Z_{i} \gg 1$, in which electron-electron collisions may be neglected. A simple solution of the constraint equation, Eq. (5.106), may be obtained, in this case. The results are, presumably, qualitatively similar to those of the more general case $Z_{i} \sim 1$, although the latter have not been calculated, except for large aspect ratio. An analysis of the Lorentz model for general geometry was first given by Rutherford (1970).

By using $C_{e} \simeq \nu_{e i} \mathcal{L}$, with $\nu_{e i}$ and $\mathscr{L}$ given by Eqs. (5.4) and (5.6), the constraint equation, Eq. (5.106), becomes

$$
\frac{\partial}{\partial \lambda} \lambda\left[\frac{\partial G_{1 e}}{\partial \lambda} \oint d \theta \xi-\frac{v f_{e 0} B_{0}}{2\left|\Omega_{e p}\right|} \oint d \theta\right]=0
$$

The integral $\oint d \theta$ equals $2 \pi$ for untrapped particles, and zero for trapped particles. Hence,

$$
\frac{\partial g_{1 e}^{(0)}}{\partial \lambda}=-\frac{v f_{e 0} B_{0}}{2 \mid \Omega_{e p}}\left[\frac{1}{\xi}-\frac{H\left(h_{\min }-\lambda B_{0}\right)}{\langle\xi\rangle}\right],
$$

where $H(x)=0$ if $x<0$ or 1 if $x>0$. The function $g_{1 e}^{(0)}$ is obtained after one further integration.

This result can be interpreted in the following way. Eq. (5.103) gives a result of the guiding-center trajectories passing through regions with different particle densities. The distribution function would be constant on a drift surface, in the absence of collisions, and this leads to the first term on the right hand side, after expanding in the poloidal gyroradius [c.f. Eq. (3.104)]. A second term $G_{1 e}$ must be added, so that the time-averaged effect of collisions on the distribution function is zero, as is required for a steady state. The constraint equation, Eq. $(5.106)$ thus states that this time average, over a particle bounce or transit, is zero, and this determines the function $G_{1 e}$. For trapped particles, which spend the same amounts of time with $v_{11}<0$ and $v_{\|}>0$, the time-averaged effect of collisions on the collisionless term is zero, so we must also have $G_{1 e}=0$ : there is no correction to the collisionless result that the distribution function is constant on a drift surface. For untrapped particles, on the other hand, the only way in which the time-averaged collision term can be zero is for the distribution function itself to be nearly zero. Thus, the two terms on the right-hand side of Eq. $(5.116)$ tend to cancel; only for the trapped and nearly trapped particles is the difference between these two terms significant.

The following explicit solutions for the other $g_{n e}^{(0)}$ 's may be obtained in a similar way

$$
\frac{\partial g_{n e}^{(0)}}{\partial \lambda}=\frac{v f_{e 0}}{2}\left[\frac{\gamma_{n} B}{\xi}-\frac{\left\langle\gamma_{n} B\right\rangle H\left(h_{\min }-\lambda B_{0}\right)}{\langle\xi\rangle}\right],
$$

where the $\gamma_{n}$ 's are defined by Eq。(5.114)。 Remarks similar to those given for the $n=1$ result, hold also for the $n=2$ result . The $n=3$ and $n=4$ results give the effect of the parallel electric field and the friction due to the ion flow term $\mu_{i}$. The interpretation of these latter two results is similar, so we shall only discuss the $n=3$ term. The first term on the right-hand side of Eq. $(5.115)$ is the negative of the Spitzer distribution function [except for factors of $E_{11}$ and $h$, which are accounted for in the definition of $g_{3 e}$, Eq. (5.69).] Comparing with Eq. (5.42), we may thus identify $G_{3 e}$ with $f_{e 1} / E_{\| 1}$, when all other driving terms except $E_{\|}$are absent. [For purposes of interpretation, we ignore the distinction between $E_{11}$ and $B\left\langle E_{\| 1} B\right\rangle /\left\langle B^{2}\right\rangle$.] The time-averaged effect of collisions, on the difference between the distribution function $f_{e 1}$ and the Spitzer function (proportional to $E_{\| 1}$ ), must be zero. For the trapped electrons, the time-averaged effect of $E_{\|}$is zero, since the work done by $E_{\|}$ on one-half of the bounce period is cancelled by the work on the other half. Thus, the distribution function $f_{e 1}$ must be zero for the trapped electrons; they are not required to have a net flow velocity (and hence a net momentum transfer to the ions), in order to balance the electric field, since the time-averaged effect of the latter is zero. For the untrapped electrons, the distribution function is not zero, since friction with the ions must approximately balance the electric field. The difference between the distribution function and the Spitzer function, as inferred from Eq. (5.117), thus tends to be small for the well-untrapped electrons.

In order to calculate the transport coefficients, we must first express the inner products $\left(\alpha_{m}, g_{n e}\right)$ in terms of the zeroth-order in collision frequency approximations, the $g_{n e}^{(0)}$ 's. Since the $\alpha_{m}$ 's are even in $\sigma(=\operatorname{sign} \xi)$ we have

$$
\left(\alpha_{m}, g_{n e}\right) \simeq\left\langle\int d^{3} v \alpha_{m} g_{n e}^{(1)}\right\rangle,
$$

where we have noted that $g_{n e}^{(0)}$ does not contribute, being an odd function. Next, we note that the $\alpha_{n}$ 's may be written in the form given by Eq. (5.114), and by integrating by parts in the $\theta$ average, we obtain

$$
\left(\alpha_{m}, g_{n e}\right)=-\left\langle\int d^{3} v\left(v_{\|} \gamma_{m}\right) v_{\|} \hat{n} \cdot \nabla g_{n e}^{(1)}\right\rangle \text {. }
$$

Finally, we use the first-order equation, Eq. (5.105), to write this as

$$
\left(\alpha_{m}, g_{n e}\right)=-\left\langle\int d^{3} v v_{\|} \gamma_{m} C_{e} g_{n e}^{(0)}\right\rangle
$$

For the special case under consideration, when $C_{e}$ $=\nu_{e i} \mathcal{L}, \mathrm{Eq} 。(5.119)$ becomes

$$
\left(\alpha_{m}, g_{n e}\right)=-\left\langle\int d^{3} v\left(v_{\|} \gamma_{m}\right) \nu_{e i} \frac{2 \xi}{B} \frac{\partial}{\partial \lambda} \lambda \xi \frac{\partial g_{n e}^{(0)}}{\partial \lambda}\right\rangle,
$$

into which we may substitute the above expressions for $\partial g_{n e}^{(0)} / \partial \lambda$ 。 After integrating in $v$, we obtain, $\mathrm{e}_{\circ} \mathrm{g}$,

$$
\left(\alpha_{1}, g_{1 e}\right)=-\frac{3}{8} n_{e} \frac{\rho_{e p}^{2}}{\tau_{e}} I_{11}
$$

where

$$
\rho_{e p}^{2} \equiv v_{\text {the }}^{2} / \Omega_{e p}^{2}=2 m_{e} c^{2} T_{e} / e^{2} B_{\mathrm{po}}^{2},
$$

and

$$
\begin{aligned}
I_{11} & \equiv\left\langle\int_{0}^{h} d \lambda^{\prime} \cdot 2 h \xi \frac{\partial}{\partial \lambda^{\prime}} \lambda^{\prime}\left[1-\frac{\xi H\left(h_{\min }-\lambda^{\prime}\right)}{\langle\xi\rangle}\right]\right\rangle \\
& =\left\langle\int_{h_{\min }}^{h} \frac{\lambda^{\prime} d \lambda^{\prime}}{\xi}\right\rangle+\int_{0}^{h_{\min }} \lambda^{\prime} d \lambda^{\prime}\left[\left\langle\frac{1}{\xi}\right\rangle-\frac{1}{\langle\xi\rangle}\right],
\end{aligned}
$$


where $\lambda^{\prime} \equiv \lambda B_{0}$. The second expression for $I_{11}$ was obtained by integrating by parts in $\lambda^{\prime}$, and is a convenient form for calculations with specific geometries; for the case of large aspect ratio and circular cross section, discussed in Sec.VI, we have

$$
I_{11} \simeq 1.38(2 \epsilon)^{1 / 2}+O\left(\epsilon^{3 / 2}\right),
$$

where $\epsilon \equiv r / R_{0}$ is the inverse aspect ratio. Although the assumption of a single minimum in $B$ is implicit in Eq. (5.123), it is easily generalized to include multiple trapped particle regions (in noncircular geometries) as pointed out by Frieman (1970).

The first expression for $I_{11}$ gives us information about the relative contributions to the integral which come from different regions of phase space. [Recall that Eq. $(5.120)$ is a result of calculating the inner product directly with the first-order (in collision frequency) part of the distribution function, without integrating by parts in $\lambda$.] Besides the contributions from the trapped $\left(h_{\min }<\lambda^{\prime} \leq h_{\max }\right)$ and untrapped $\left(0 \leq \lambda^{\prime}<h_{\min }\right)$ regions, there is a finite contribution from the boundary $\lambda^{\prime}=h_{\min }$ between these two regions. Mathematically, it arises from differentiating the step function in Eq. (5.116), which gives a delta function $\delta\left(\lambda^{\prime}-h_{\min }\right)$ in the integrand. This delta function is actually part of the first-order (in collision frequency) distribution function, which is even in $v_{11}$. It represents the tendency for the marginally trapped orbits to be overpopulated, due to the discontinuity in the diffusion flux (proportional to $\partial g / \partial \lambda$ ) across the boundary $\lambda^{\prime}=h_{\min }$. [We thus see that the random walk picture of Sec. III is oversimplified.]

Actually, the banana regime approximation itself breaks down near the trapping boundary. The small expansion parameter is not given by Eq. (5.100), in general, but is $\nu_{e i} \tau_{b}\left(\lambda^{\prime}\right)$, where $\tau_{b}$ is the "bounce time," which becomes logarithmically infinite as $\lambda^{\prime}$ approaches $h_{\text {min }}$. The marginally trapped particles are most susceptible to perturbation by collisions; in fact, they make the predominant contribution to the particle diffusion, in the large aspect ratio limit.

When the boundary layer near $\lambda^{\prime}=h_{\min }$ is treated more accurately, (Hinton and Rosenbluth, 1973) important corrections to the transport coefficients are obtained. The delta function is replaced by a function with a finite width, of order $\left(\nu_{e i} q R / v\right)^{1 / 2}$, which is also shifted away from the trapping boundary, into the trapped region, by an amount of this same order. This shift decreases the effective value of $m_{e} v_{11}$, for the particles contributing to diffusion, and thus decreases the transport coefficients. This boundary-layer correction is discussed in more detail in Sec. VI.D.

All of the other transport coefficients may be obtained by evaluating the integrals in Eq. (5.120). The results are

$$
\begin{aligned}
& \left(\alpha_{1}, g_{2 e}\right)=\frac{9}{16} \frac{n_{e} \rho_{e p}^{2}}{\tau_{e}} I_{11}, \quad\left(\alpha_{2}, g_{2 e}\right)=-\frac{39}{32} \frac{n_{e} \rho_{e p}^{2}}{\tau_{e}} I_{11}, \\
& \left(\alpha_{1}, g_{3 e}\right)=-\frac{3}{4} \frac{n_{e} c}{B_{\mathrm{po}}} I_{13}, \quad\left(\alpha_{2}, g_{3 e}\right)=0, \\
& \left(\alpha_{1}, g_{4 e}\right)=-\frac{3}{8} \frac{n_{e} \rho_{e p}^{2}}{\tau_{e}} I_{13}, \quad\left(\alpha_{2}, g_{4 e}\right)=\frac{9}{16} \frac{n_{e} \rho_{e p}^{2}}{\tau_{e}} I_{13},
\end{aligned}
$$

$$
\left(\alpha_{3}, g_{4 e}\right)=-\frac{3}{4} n_{0} \frac{c}{B_{\mathrm{po}}} I_{33}, \quad\left(\alpha_{4}, g_{4 e}\right)=-\frac{3}{8} \frac{n_{e} \rho_{e p}^{2}}{\tau_{e}} I_{33},
$$

and

$$
\left(\alpha_{3}, g_{3 e}\right)=-\frac{8}{\pi} \frac{n_{e} e^{2} \tau_{e}}{m_{e} T_{e}} I_{33},
$$

where $I_{11}$ is given by Eq. $(5.123)$, and

$$
\begin{aligned}
& I_{13}=\left\langle\frac{1}{h^{2}} \int_{h_{\min }}^{h} \frac{\lambda^{\prime} d \lambda^{\prime}}{\xi}\right\rangle-\int_{0}^{h_{\min }} \lambda^{\prime} d \lambda^{\prime}\left[\left\langle\frac{1}{h^{2} \xi}\right\rangle-\frac{\left\langle 1 / h^{2}\right\rangle}{\langle\xi\rangle}\right], \\
& I_{33}=\left\langle\frac{1}{h^{4}} \int_{h_{\min }}^{h} \frac{\lambda^{\prime} d \lambda^{\prime}}{\xi}\right\rangle-\int_{0}^{h_{\min }} \lambda^{\prime} d \lambda^{\prime}\left[\left\langle\frac{1}{h^{4} \xi}\right\rangle-\frac{\left\langle 1 / h^{2}\right\rangle^{2}}{\langle\xi\rangle}\right] .
\end{aligned}
$$

In the large aspect-ratio, circular cross section case, we have

$$
I_{13} \simeq I_{33} \simeq I_{11} \simeq 1.38(2 \epsilon)^{1 / 2},
$$

with error terms of order $\epsilon^{3 / 2}$, where $\epsilon \equiv r / R_{0}$.

In evaluating the integrals which contain $\alpha_{3}$ (or $\gamma_{3}$ ), we have used the result that, for the Lorentz model, the solution of Eq. (5.44) is

$$
f_{s e}=-\left(e / T_{e}\right) f_{e 0} / \nu_{e i}\left(\text { for } Z_{i} \gg 1\right) \text {. }
$$

Note that the integrals containing $\alpha_{3}$, which determine the current density, Eq. (5.72), could also be obtained more directly by using Eq. (5.65). The latter evaluation enables us to locate the current carriers in phase space.

We now attempt an interpretation of these results. The definition of the inner products is, of course, given by Eqs. (5.70)-(5.72), where the $A_{n e}$ 's are defined in Eqs. (5.56)-(5.58). In addition to the dependence on the pressure gradients, the particle flux is linear in the temperature gradient, the coefficient of proportionality being $\left(\alpha_{1}, g_{2 e}\right)$. This effect is similar to the effect of a temperature gradient on the classical diffusion flux [c.f., Eq. (4.18)]. It is due to the velocity-dependence of the collision frequency $\nu_{e i}$. A parallel friction force arises because of the noncancellation of momentum, transferred to the ions, by electrons on banana orbits passing through regions of different temperatures.

The coefficient $\left(\alpha_{2}, g_{2 e}\right)$ is just the electron thermal conductivity. The Onsager relation $\left(\alpha_{2}, g_{1 e}\right)=\left(\alpha_{1}, g_{2 e}\right)$ tells us that the pressure gradients also drive a heat flux, due to the velocity dependence of $\nu_{e i}$.

There is a radially inward particle flux driven by the parallel electric field, with the coefficient of proportionality $\left(\alpha_{1}, g_{3 e}\right)$. This is the "trapped particle pinch effect," discovered independently by Ware (197.0) and Galeev (1971). Its order of magnitude is consistent with the simple argument given by Ware, that the flux is due to the bounce-averaged drifts of the trapped particles, as discussed in Sec. III. The above remarks about the role of the boundary layer apply here also, however; in fact, for large aspect ratio, the flux is mainly the diffusion of the marginally trapped particles in the boundary layer (Rutherford, et al., 1970).

A similar effect in the heat flux is absent, in the Lorentz model: note that $\left(\alpha_{2}, g_{3 e}\right)=0$. In the corresponding 
integral expression given by Eq. (5.120), the factor $\nu_{e i}$ cancels out of the integrand, leaving an energy-dependent term proportional to $v^{2}\left(\frac{1}{2} m_{e} v^{2}-\frac{5}{2} T_{e}\right)$. This integrates to zero: the average electron energy carried inward in the trapped particle pinch effect is exactly $\frac{5}{2} T_{e}$ 。 When the electron-electron collisions are included, there is no longer an exact cancellation.

Because of the Onsager relations, we have a term proportional to the pressure gradients, in the parallel current density, whose coefficient is $\left(\alpha_{3}, g_{1 e}\right)=\left(\alpha_{1}, g_{3 e}\right)$ 。 Such a term was first pointed out by Galeev (1971), and independently by Bickerton, Connor and Taylor (1971). The corresponding current is called the "bootstrap current," since it makes possible the idea of a "bootstrap tokamak," operating in a steady state with $E_{T}=0$. This possibility was first investigated by Bickerton, et al., (1971), and independently by Kadomtsev and Shafranov (1972)。A limit, on plasma pressure in a tokamak, was also pointed out independently by Bickerton, et al., (1971), and by Galeev and Sagdeev (1971). This limit arises from the condition that the bootstrap current not violate the Kruskal-Shaf ranov criterion for magnetohydrodynamic stability, Eq. (2.51).

The origin of this "bootstrap" current is in the banana orbits of the trapped electrons. They set up a parallel flow, in a manner analogous to the perpendicular diagmagnetic flow due to the particles gyrating around their guiding centers. Actually, the untrapped electrons carry most of the current, in the large aspect-ratio limit, when the fraction of trapped particles is small. This is due to the fact that electron-ion collisions force the untrapped particle distribution to be continuous with the trapped distribution; the parallel flow set up by the banana orbits is retained, to some extent, by the trapped particles even after they have diffused into the untrapped region of phase space.

The terms in Eqs. (5.70)-(5.72) which are proportional to $A_{4 e}$ may be interpreted as the result of the additional friction on the electrons resulting from the ion flow term proportional to $\mu_{i}$, in Eq. (5.46). The coefficient $\left(\alpha_{4}, g_{4 e}\right)$ gives the reaction back on the ions, through friction with the electrons, and enters the self-consistent determination of the forces $A_{1 i}, A_{4 e}$, Eqs. (5.75) and (5.76).

The coefficient of the parallel electric field in Eq. (5.72) is $\left(\alpha_{3}, g_{3 e}\right)$, which gives the reduction in the electrical conductivity, due to the fact that the trapped electrons cannot carry any of the Ohmic current. This result was first obtained by Hinton and Oberman (1969).

\section{Ion distribution function}

The perturbation analysis of Eq. (5.81), in the case $n=2$, is very similar to that given for the electron equation. The constraint equation is found to be

$$
\oint \frac{d \theta B}{v_{11}} C_{i i} g_{2 i}^{(0)}=0
$$

where

$$
g_{2 i}^{(0)}=-\left(v_{11} h / \Omega_{i p}\right)\left(v^{2} / v_{\mathrm{th} i}^{2}-5 / 2\right) f_{i 0}+G_{2 i},
$$

with $\partial G_{2 i} / \partial \theta=0$. The ion thermal conductivity is given, in terms of the solution of this equation, by

$$
-\left(\beta_{2}, g_{2 i}\right)=\left\langle\int d^{3} v \frac{v_{\text {॥ }} h}{\Omega_{i p}}\left(v^{2} / v_{\text {th } i}^{2}-5 / 2\right) C_{i i} g_{2 i}^{(0)}\right\rangle .
$$

The banana-regime variational principle is

$$
\left[Q_{22}^{(i)}\right]=-\left\langle\int d^{3} v \hat{g}_{2 i}^{(0)} C_{i i} g_{2 i}^{(0)}\right\rangle
$$

the minimum value of which is the thermal conductivity

$$
Q_{22}^{(i)}=-\left(\beta_{2}, g_{2 i}\right) \text {. }
$$

In the case $n=1$, the perturbation analysis is somewhat different. The leading term is of order $\left(\nu_{i i} q R / v\right)^{-1}$, and satisfies

$$
v_{\| 1} \hat{n} \cdot \nabla g_{1 i}^{(-1)}=0 \text {. }
$$

It is determined by the constraint equation

$$
\oint \frac{d \theta B}{v_{11}} C_{i i} g_{1 i}^{(\text {(r) })}=\frac{2 \Omega_{i p} B_{0} f_{i 0}}{n_{i} v_{\mathrm{th} i}^{2}\left\langle B^{2}\right\rangle} \oint B^{2} d \theta,
$$

where $\oint B^{2} d \theta$ equals zero for the trapped ions, and $2 \pi\left\langle B^{2}\right\rangle$ for the untrapped ones. A variational principle is easily constructed but will not be given here. The problem of determining the function $g_{1 i}^{(-1)}$, has not been considered in the literature. It is needed only to calculate $\left(\beta_{1}, g_{1 i}\right)$, which helps determine the forces $A_{1 i}, A_{4 e}$ self-consistently. It is not needed at all in the weak-coupling approximation, which is most commonly used.

\section{E. Collisional regime}

\section{Introduction}

When the particle collision frequencies are much higher than their bounce or transit frequencies, the collision terms dominate the kinetic equations, Eqs. (5.80) and (5.81). A perturbation method (Rutherford, 1970; Frieman, 1970) which is similar to the Chapman-Enskog method of the kinetic theory of gases, can then be used. Since the method is very similar to that used in Sec. IV, the discussion in this section will be somewhat brief. We will see that more information can be obtained from the kinetic theory than was available using the macroscopic approach in Sec. IV.D.

\section{Electron transport}

We begin by expanding $g_{n e}$ in powers of $v / \nu_{e i} q R(\ll 1)$, beginning with a term of order $\left(v / \nu_{e i} q R\right)^{-1}$, which is generally necessary:

$$
g_{n e}=g_{n e}^{(-1)}+g_{n e}^{(0)}+g_{n e}^{(1)}+\cdots .
$$

Equation (5.80) becomes, through the first three orders

$$
\begin{aligned}
& C_{e} g_{n e}^{(-1)}=0 \\
& C_{e} g_{n e}^{(0)}=\overrightarrow{\mathrm{v}}_{\|} \cdot \nabla g_{n e}^{(-1)}, \\
& C_{e} g_{n e}^{(1)}=\overrightarrow{\mathrm{v}}_{\|} \cdot \nabla\left(g_{n e}^{(0)}+v_{\|} \gamma_{n} f_{e 0}\right),
\end{aligned}
$$

where $\gamma_{n}$ is defined by Eq. (5.114). Equation (5.138) implies that $g_{n e}^{(-1)}$ is the perturbation of a Maxwellian due to pressure and temperature perturbations

$$
g_{n e}^{(-1)}=\left[a_{n}+\left(v^{2} / v_{\text {the }}^{2}-5 / 2\right) b_{n}\right] f_{e 0} .
$$

Equation (5.139) is then the Spitzer problem [c.f., Sec. IV.C] and the parallel gradients of pressure and temperature drive parallel current and heat flows deter- 
mined by its solution

$$
\begin{aligned}
& -\int d^{3} v v_{\|} g_{n e}^{(0)}=K_{11} \nabla_{\|} a_{n}+K_{12} \nabla_{\|} b_{n}, \\
& -\int d^{3} v v_{\|}\left(v^{2} / v_{\text {the }}^{2}-5 / 2\right) g_{n e}^{(0)}=K_{12} \nabla_{\|} a_{n}+K_{22} \nabla_{\|} b_{n},
\end{aligned}
$$

where the $K_{m n}$ 's are the parallel transport coefficients, defined by Eq. (4.77) and Table II. Equation (5.140) has solubility conditions which determine the function $g_{n e}^{(-1)}$ uniquely

$$
\begin{aligned}
& \overrightarrow{\mathrm{B}} \cdot \nabla \int d^{3} v \frac{v_{\|}}{B}\left(g_{n e}^{(0)}+v_{\|} \gamma_{n} f_{e 0}\right)=0, \\
& \overrightarrow{\mathrm{B}} \cdot \nabla \int d^{3} v \frac{m_{e} v^{2}}{2} \frac{v_{\|}}{B}\left(g_{n e}^{(0)}+v_{\|} \gamma_{n} f_{e 0}\right)=0 .
\end{aligned}
$$

The solution of these differential equations may be written as

$$
\begin{gathered}
\int d^{3} v v_{\|} g_{n e}^{(0)}=-\int d^{3} v v_{\|}^{2} \gamma_{n} f_{e 0}+k_{1}(\rho) / h \\
\int d^{3} v v_{\|}\left(v^{2} / v_{\text {the }}^{2}-5 / 2\right) g_{n e}^{(0)}=-\int d^{3} v\left(v^{2} / v_{\text {the }}^{2}-5 / 2\right) v_{\| 1}^{2} \gamma_{n} f_{e 0} \\
+k_{2}(\rho) / h
\end{gathered}
$$

where the functions $k_{1}(\rho)$ and $k_{2}(\rho)$ are determined by multiplying Eqs. (5.142), (5.143), (5.146), and (5.147) by $B(\propto 1 / h)$ and averaging over a magnetic surface using Eq. (2.53)

$$
\begin{aligned}
& k_{1}(\rho)\left\langle h^{-2}\right\rangle=\left\langle\int d^{3} v v_{\|}^{2}\left(\gamma_{n} / h\right) f_{e 0}\right\rangle, \\
& k_{2}(\rho)\left\langle h^{-2}\right\rangle=\left\langle\int d^{3} v\left(\frac{v^{2}}{v_{\text {the }}^{2}}-\frac{5}{2}\right) v_{\|}^{2}\left(\gamma_{n} / h\right) f_{e 0}\right\rangle .
\end{aligned}
$$

Equations (5.142) and (5.143) may now be solved for $\nabla_{\|} a_{n}$ and $\nabla_{11} b_{n}$, which uniquely determines $g_{n a}^{(-1)}$ under the further condition $\left\langle a_{n}\right\rangle=0=\left\langle b_{n}\right\rangle$.

The effect of the guiding-center drifts, and the resulting perpendicular flows, contained in $\gamma_{1}$ and $\gamma_{2}$, is to require parallel return flows of particles and heat, described by $g_{1 e}^{(0)}$. Equations (5.144) and (5.145) express the requirement that the divergence of the net flows be zero, to this order in the gyroradius. In a collisiondominated plasma, the existence of parallel flows requires parallel gradients of pressure and temperatureas given by Eqs. (5.142) and(5.143). The flux-surface averaged radial fluxes are then determined by these parallel gradients. For example, when there are more particles at the "top" of the magnetic surface (see Fig. 6) drifting up and hence away from the magnetic axis than are at the "bottom" drifting toward the magnetic axis, the radial surface-averaged particle flux is positive.

The inner products may now be calculated using Eq. (5.118) with $g_{n \dot{e}}^{(1)}$ replaced by $g_{n e}^{(-1)}$, which is given by Eq. (5.141)

$$
\left(\boldsymbol{\alpha}_{m}, g_{n e}\right)=-\left\langle\int d^{3} v v_{\|}^{2} \gamma_{m} f_{e 0}\left[\nabla_{\|} a_{n}+\left(v^{2} / v_{\text {the }}^{2}-5 / 2\right) \nabla_{\|} b_{n}\right]\right\rangle .
$$

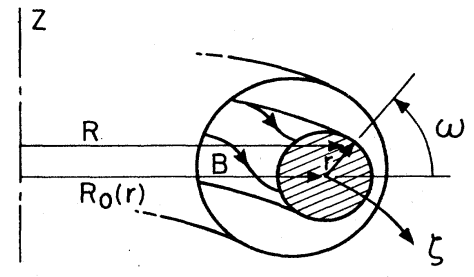

FIG. 6. Toroidal coordinate system, showing its relation to the cylindrical coordinates $R, Z, \zeta$.

After solving Eqs. (5.142) and (5.143) for $\nabla_{\| 1} a_{n}$ and $\nabla_{\| 1} b_{n}$, with the left-hand sides of those equations given by Eqs. (5.146)-(5.149), and substituting into Eq. (5.150), we obtain

$$
\begin{aligned}
& \left(\alpha_{1}, g_{1 e}\right)=-\frac{1}{2} \frac{\kappa_{22}}{\kappa} n_{e}\left(\rho_{e p}^{2} / \tau_{e}\right)\left[\left\langle h^{2}\right\rangle-\left\langle h^{-2}\right\rangle^{-1}\right], \\
& \left(\alpha_{1}, g_{2 e}\right)=\frac{5}{4} \frac{\kappa_{12}}{\kappa} n_{e}\left(\rho_{e p}^{2} / \tau_{e}\right)\left[\left\langle h^{2}\right\rangle-\left\langle h^{-2}\right\rangle^{-1}\right], \\
& \left(\alpha_{2}, g_{2 e}\right)=-\frac{25}{8} \frac{\kappa_{11}}{\kappa} n_{e}\left(\rho_{e p}^{2} / \tau_{e}\right)\left[\left\langle h^{2}\right\rangle-\left\langle h^{-2}\right\rangle^{-1}\right],
\end{aligned}
$$

where $\rho_{e p}^{2} \equiv 2 T_{e} / m_{e}\left|\Omega_{e p}\right|$, and the $\kappa$ 's are given in Table II. When these expressions are substituted into Eqs. (5.70) and (5.71), the results agree with the axisymmetric limit of Eq. (4.98) and (4.99), with $I \equiv R_{0} B_{T 0}$ independent of $\theta$. [The terms involving the electric field, which appear in Eqs. (4.98) are not contained in $\Gamma_{e}$, as defined by Eq. (5.62), since that definition differs from the left-hand side of Eq. (4.98).]

The factor in square brackets in Eqs. (5.151)-(5.153) is positive, which can be seen by writing it as $\left\langle\left(h-h^{-1}\left\langle h^{-2}\right\rangle^{-1}\right)^{2}\right\rangle$. This factor is smaller than the factor $I_{11}$ which appears in Eq. (5.121), the banana regime diffusion coefficient; by multiplying the identity

$$
\left\langle\xi^{-1} h^{-4}\right\rangle-\left\langle h^{-2}\right\rangle^{2} /\langle\xi\rangle=\left\langle\left[\xi^{-1 / 2} h^{-2}-\xi^{1 / 2} \frac{\left\langle h^{-2}\right\rangle}{\langle\xi\rangle}\right]^{2}\right\rangle>0
$$

by $\lambda$ and integrating over $\lambda$, we find, on comparison with Eq. (5.123), that

$$
I_{11}>\frac{4}{3}\left[\left\langle h^{2}\right\rangle-\left\langle h^{-2}\right\rangle^{-1}\right] \text {. }
$$

Thus, the enhancement over classical diffusion is always greater in the banana regime than in the collisional regime (Rutherford, 1970). In the large aspect-ratio, circular cross section case, discussed in more detail in Sec. VI, we find

$$
\left[\left\langle h^{2}\right\rangle-\left\langle h^{-2}\right\rangle^{-1}\right] \simeq 2 \epsilon^{2},
$$

where $\epsilon=r / R_{0} \ll 1$, which is to be compared with the larger factor $I_{11} \simeq 1.38(2 \epsilon)^{1 / 2}$.

The other inner products, with $m$ or $n$ equal to 3 or 4 , are zero to this order in the collision frequency, since $\gamma_{3}$ and $\gamma_{4}$ are proportional to $1 / h$, and the right-hand sides of Eqs. (5.146) and (5.147) vanish identically. We must therefore procede to higher-order. Since $g_{3 e}^{(-1)}$ $=g_{4 e}^{(-1)}=0$, the solution of Eq. (5.139) is

$$
g_{n e}^{(0)}=\left[c_{n}+\left(v^{2} / v_{\text {the }}^{2}-5 / 2\right) d_{n}\right] f_{e 0}
$$

for $n=3$ and 4 ; using the solubility condition for the equation for $g_{n e}^{(2)}$, we find $c_{n}=d_{n}=0$. 
The equation for $g_{4 e}^{(1)}$ is therefore

$$
\begin{aligned}
C_{e} g_{4 e}^{(1)} & =-\overrightarrow{\mathrm{v}}_{\|} \cdot \nabla\left(v_{\|} / h\right) f_{e 0} /\left|\Omega_{e p}\right| \\
& =-\left(v^{2} /\left|\Omega_{e p}\right|\right) \nabla_{\|}(1 / h)\left(\frac{3}{2} \xi^{2}-\frac{1}{2}\right) f_{e 0},
\end{aligned}
$$

which is equivalent to Eq. (4.57) with the right-hand side proportional to $P_{2}(\xi) \equiv \frac{3}{2} \xi^{2}-\frac{1}{2}$. This equation has been approximately solved by Braginskii (1965). By making use of his results, we find

$$
\left(\alpha_{4}, g_{4 e}\right)=-\frac{9}{2} \eta_{0}^{e}\left(T_{e} / m_{e}^{2} \Omega_{e p}^{2}\right)\left\langle\left[\nabla_{\|}(1 / h)\right]^{2}\right\rangle,
$$

where $\eta_{0}^{e}=0.73 n_{e} T_{e} \tau_{e}$ (for $Z_{i}=1$, for example) is the coefficient of parallel electron viscosity.

Note that this transport coefficient decreases with increasing collision frequency. The same is true for the coefficients $\left(\alpha_{m}, g_{4 e}\right)$ for $m=1,2,3$; we find, for example

$$
\left(\alpha_{1}, g_{4 e}\right)=\frac{3}{2} \eta_{0}^{e}\left(T_{e} / m_{e}^{2} \Omega_{e p}^{2}\right)\left\langle\left(\nabla_{\|} \ln h\right)^{2}\right\rangle .
$$

For this reason, the contributions of the force $A_{4 e}$ in the electron particle and heat fluxes, given by Eqs. (5.70) and (5.71), are negligible, of order $\left(v_{\mathrm{the}} \tau_{e} / q R\right)^{2} \mathrm{com}-$ pared with the terms containing $A_{1 e}$ and $A_{2 e}$. Also for this reason, the ion heat flux driven by friction with the electrons, given by Eqs. (5.84) and (5.85), is negligible [we will find, subsequently, that $\left(\beta_{1}, g_{2 i}\right)$, which appears in Eq. (5.85) is of order unity].

The equation for $g_{3 e}^{(1)}$ is similar to Eq. (5.154), but has $-1 /\left|\Omega_{e p}\right|$ replaced by the Spitzer function $\hat{f}_{s e}$ on the righthand side. Its solution has not been determined, to our knowledge. We note that, since $f_{s e} \sim 1 / \nu$, the coefficients $\left(\alpha_{m}, g_{3 e}\right)$, for $m=1,2,4$, must be proportional to $\tau_{e}^{2}$, while $\left(\alpha_{3}, g_{3 e}\right)$ must be proportional to $\tau_{e}^{3}$, in the collisional regime. Consequently, the right-hand side of Eq. (5.72) is negligible, in the collisional regime.

\section{Ion transport}

After expanding in powers of $v / \nu_{i i} q R(\ll 1)$ where $\nu_{i i}$ is the ion collision frequency for $90^{\circ}$ scattering, Eq. (5.81) becomes, through the first three orders

$$
\begin{aligned}
& C_{i i} g_{n i}^{(-1)}=0, \\
& C_{i i} g_{n i}^{(0)}=\overrightarrow{\mathrm{v}}_{\| 1} \cdot \nabla g_{n i}^{(-1)}, \\
& C_{i i} g_{n i}^{(1)}=\overrightarrow{\mathrm{v}}_{\| 1} \cdot \nabla g_{n i}^{(0)}+\beta_{n} f_{i 0},
\end{aligned}
$$

where the $\beta_{n}$ are defined by Eq. (5.55). The general solution of Eq. (5.157) is

$$
g_{n i}^{(-1)}=\left[a_{n} B+b_{n} v_{\| 1} B+c_{n}\left(v^{2} / v_{\text {thi }}^{2}-5 / 2\right)\right] f_{i 0},
$$

so that Eq. (5.158) is again a general Spitzer problem. The conditions of solubility of this equation are $\overrightarrow{\mathrm{B}} \cdot \nabla a_{n}$ $=\overrightarrow{\mathrm{B}} \cdot \nabla b_{n}=0$. The conditions of solubility of Eq. (5.159) are different in the two cases $n=1$ and $n=2$, so we consider them separately.

We consider first the case $n=2$. Since $\beta_{2}$ has the form $\beta_{2}=v_{11} \hat{n} \cdot \nabla(\cdots)$, the analysis is very similar to that for the electron thermal conductivity given above. The solu bility conditions which are obtained from conservation of particles and energy, give $a_{2}=0$. The momentum conservation condition yields $b_{2}=0$; thus, as noted in Eq. (4.49), the parallel flow velocity can not be large in the collision frequency parameter. Eq. (5.158) for $n=2$ then reduces to an equation solved by Braginskii (1965). By making use of his result for the parallel ion thermal con- ductivity, and following the above method, we find

$$
-\left(\beta_{2}, g_{2 i}\right)=0.8 n_{i}\left(\rho_{i \rho}^{2} / \tau_{i}\right)\left[\left\langle h^{2}\right\rangle-\left\langle h^{-2}\right\rangle^{-1}\right] ;
$$

compare Eq. (4.95), The ion guiding -center drifts represent a perpendicular heat flux, which must be compensated by a parallel heat flux, to make the total flux diver gence free. In a collision-dominated plasma, this parallel heat flux requires a parallel temperature gradient, and this is directly related to the surface-averaged radial heat transport, as given by Eq. (5.161).

The function $g_{2 i}^{(0)}$ is not uniquely determined, without proceeding to higher-order in the short mean-free-path expansion. An additional term, proportional to $v_{\mathrm{ll}} f_{i \mathrm{0}}$ may be present, without affecting the left-hand side of Eq. (5.158). This term is needed for the calculation of $\left(\beta_{1}, g_{2 i}\right)$, and hence $\mu_{i}$, from Eq. (5.83) (in the weak-coupling approximation), which helps determine the ion parallel velocity, in Eq. (5.46). This problem was solved by Hazeltine (1974). He used a variational method to solve Eq. (5.159), for $n=2$, and then used the solubility condition on the equation for $g_{2 i}^{(2)}$ to determine $g_{2 i}^{(0)}$ uniquely. His result can be expressed as

$$
-\left(\beta_{1}, g_{2 i}\right)=\frac{1.8}{\left\langle h^{-2}\right\rangle}+0.27 \frac{\left\langle\left(\nabla_{11} \ln h\right)^{2}\right\rangle}{\left\langle\left(\nabla_{\| 1} h^{-1}\right)^{2}\right\rangle} .
$$

In the large aspect-ratio, circular cross section limit, this reduces to $\left(\beta_{1}, g_{2 i}\right) \simeq \mathbf{- 2 . 1}$.

In the $n=1$ case, the solubility conditions corresponding to particle and energy conservation yield $a_{1}=c_{1}=0$. The momentum conservation condition gives, upon multiplying by $B$ and averaging

$$
\left\langle\nabla_{\|}(1 / h) \int d^{3} v v^{2} P_{2}(\xi) g_{2 i}^{(0)}\right\rangle=\Omega_{i p}
$$

Equation (5.158) for $n=1$ is equivalent to an equation solved by Braginskii (1965). By making use of his results in Eq. (5.163), we find the value of $b_{1}$ and finally obtain the inner product

$$
\begin{aligned}
\left(\beta_{1}, g_{1 i}^{(-1)}\right) & =B_{0} \Omega_{i p} b_{1} \\
& =-\Omega_{i p}^{2} / \frac{9}{2} \eta_{0}^{i} \frac{T_{i}}{m_{i}^{2}}\left\langle\left(\nabla_{\|} \frac{1}{h}\right)^{2}\right\rangle,
\end{aligned}
$$

where $\eta_{0}^{i}=0.96 n_{i} T_{i} \tau_{i}$ is the coefficient of parallel ion viscosity.

The friction force due to the electrons must be balanced by a viscous ion stress, which requires a nonuniform ion flow, represented by the $b_{1}$ term in Eq. $(5.160)$, as given by Eq. (5.164). This ion flow, and the friction which it leads to, causes the electrons to flow nonuniformly, resulting in an electron stress; the degree to which the electrons and ions are coupled, throughfrictional interaction is determined by $F-1$ where $F$ is defined by Eq. (5.79). Using Eqs. (5.155) and (5.164), we find $F-1=\eta_{0}^{e} / \eta_{0}^{i}$, the ratio of the parallel viscosity coefficients. For $Z_{i}=1$, for example

$$
F-1=0.54\left(m_{e} / m_{i}\right)^{1 / 2}\left(T_{e} / T_{i}\right)^{5 / 2}
$$

The weak-coupling approximation $(F-1 \ll 1)$ requires (for the hydrogen mass ratio) $T_{e} / T_{i}<5$, which is satis fied under most experimental conditions. Even if this condition were not satisfied, the only change in the above results would be the value of $\mu_{i}$ which appears in Eq. (5.46). 


\section{TRANSPORT COEFFICIENTS FOR LARGE ASPECT- RATIO TOKAMAKS}

\section{A. Geometry}

The problem of specifying the coordinate system, in a concrete way, is greatly simplified in the large aspectratio limit

$$
\epsilon \equiv r / R_{0} \ll 1 \text {. }
$$

Here $r$ is the minor radius, and $R_{0}$ the major radius; these will be defined subsequently. In this limit, many solutions of the flux surface equilibrium equations are known, the most generally useful being given as follows (Shaf ranov, 1966).

We define the toroidal coordinates $r, \omega, \zeta$ in terms of the usual cylindrical coordinates $R, Z, \zeta$ (see Fig.6) by

$$
R=R_{0}(r)+r \cos \omega, \quad Z=r \sin \omega,
$$

where the function $R_{0}(r)$ gives the distance from the symmetry axis to the center of the (circular) flux surface cross section whose minor radius is $r$. The distance to the magnetic axis is $R_{0}(0) \equiv R_{0}$. The poloidal flux function is then

$$
\psi=R_{0} \int_{0}^{r} d r B_{\mathrm{po}}(r)+O\left(\epsilon^{2}\right),
$$

where $B \mathrm{po}(r)$ is the effective poloidal field magnitude, (the time dependence is not written explicitly here) which must be related to the pressure gradient by

$$
\frac{1}{r^{2}} \frac{d}{d r}\left(\frac{r^{2}}{2} B_{\mathrm{po}}^{2}\right)+\frac{1}{R_{0}^{2}} \frac{d}{d r}\left(\frac{1}{2} I_{\mathrm{o}}^{2}\right)=-4 \pi \frac{d P}{d r} .
$$

Here we have written the toroidal field as

$$
B_{\mathrm{T}} \simeq I_{0}(r) / R \text {. }
$$

Equation (6.4) is the equation of pressure balance in a cylindrical plasma of circular cross section, and is a cons equence of the equilibrium equation, Eq. (2.90), to zeroth order in $\epsilon$. A term of order $\epsilon$ is not present in Eq. (6.3) provided that $R_{0}(r)$ satisfies

$$
\frac{d}{d r} r B_{\mathrm{po}}^{2} \frac{d}{d r}\left[R_{0}^{2}(r) / 2\right]+r B_{\mathrm{po}}^{2}=8 \pi r^{2} \frac{d P}{d r} .
$$

That is, the relative shift, of the flux surface centers is determined by the profiles of pressure and poloidal field. In general, $d R_{0} / d r=O(\epsilon)$, provided that $\beta_{p} \equiv 8 \pi P / B_{\mathrm{po}}^{2}$, the ratio of plasma pressure to poloidal magnetic pressure, is not much larger than unity (it is typically somewhat less than one, in present tokamak experiments).

Neglecting terms of order $\epsilon^{2}$, the poloidal field is therefore given by

$$
\overrightarrow{\mathrm{B}}_{p}=\hat{e}_{\zeta} \times \nabla r\left(R_{0} / R\right) B_{\mathrm{po}}(r)=\hat{e}_{\omega} \frac{R_{0} B_{\mathrm{po}}(r)}{R\left[1+\left(d R_{0} / d \gamma\right) \cos \omega\right]},
$$

where $\hat{e}_{r}, \hat{e}_{\omega}$ are unit vectors in the $r$ and $\omega$ directions, respectively. The magnitude of the poloidal field is

$$
B_{p}=B_{\mathrm{po}}(r)\left[1-\Lambda(r)\left(r / R_{0}\right) \cos \omega\right]^{-1}+O\left(\epsilon^{2}\right),
$$

where

$$
\Lambda r / R_{0} \equiv-\left(r / R_{0}+d R_{0} / d r\right)
$$

so that the coordinate $\theta$, introduced in Sec. V.B, is related to $\omega$ by

$$
\theta=\omega-\Lambda\left(r / R_{0}\right) \sin \omega+O\left(\epsilon^{2}\right) .
$$

We will continue to work with the coordinate $\theta$; in fact, the approximation

$$
\omega \simeq \theta
$$

will be adequate.

The toroidal flux is given by

$$
\phi=B_{\mathrm{To}} \cdot \pi r^{2}+O\left(\epsilon^{2}\right),
$$

so that the minor radius coordinate $\rho$, introduced in Sec. V.B, may be identified with $r$.

\section{B. Electron transport in the banana regime 1. Large aspect-ratio expansion}

In the large aspect-ratio limit, the solution of the kinetic equations is simplified by making use of the small ness of the fraction of trapped particles, which is approximately $(2 \epsilon)^{1 / 2}$. Assuming that

$$
(2 \epsilon)^{1 / 2} \ll 1,
$$

the trapped particle region of phase space is very narrow, consisting of the small range of pitch angles such that $1-\epsilon \leq \lambda B_{0} \leq 1+\epsilon$, where $\lambda$ is the pitch-angle variable, defined by Eq. (3.63). A variational method (Rosenbluth, Hazeltine and Hinton, 1972) can then be used to calculate the transport coefficients exactly, in the limit of small $\epsilon$ (and small collision frequency).

We begin the analysis with the banana regime variational principle of Sec. V.D. We wish to minimize the functional

$$
\left[Q_{11}^{(e)}\right] \equiv-\int_{-\pi}^{\pi} \frac{d \theta}{2 \pi} \int d^{3} v \hat{g}_{1 e} C_{e} g_{1 e},
$$

subject to the constraint that $g_{l e}$ has the form given by Eq. (5.103), where $G_{1}$ is independent of $\theta$ and is an even function of $\sigma\left(\equiv \operatorname{sign} v_{11}\right)$ in the trapped particle region of phase space. [Note that we have dropped the superscript $(0)$; the functions $g_{l e}$ and $G_{1}$ are here understood to be zeroth order in collision frequency.] The minimum val ue of $\left[Q_{11}^{(e)}\right]$ then gives the desired transport coefficient, according to Eq. (5.112).

The function $G_{1}$, defined by

$$
g_{1 e}=\left(v_{11} h /\left|\Omega_{e p}\right|\right) f_{e 0}+G_{1}
$$

is assumed to have an expansion of the form

$$
G_{1}=G^{(0)}+G^{(1)}+\cdots,
$$

where the superscript gives the order, in $\epsilon^{1 / 2}$, of the derivative, $\lambda \partial G / \partial \lambda$, in the trapped particle region. $\mathrm{Be}-$ cause of our assumption concerning the smallness of this region in the $\lambda$ direction (see Fig. 5) the predominant effect of the collision operator $C_{e}$ is contained in the $\lambda$ derivatives, i.e., pitch-angle scattering is more important than the other collisional processes contained in $C_{e}$. To make use of this assumption, we write

$$
G^{(0)}=-\left(v_{11} h /\left|\Omega_{e p}\right|\right) f_{e 0}+h^{(0)} \text {. }
$$

The function $h^{(0)}$ is assumed to be "localized" in $\lambda$, in the sense that $\lambda \partial h^{(0)} / \partial \lambda$ is of order unity in the trapped 
region [much larger than $v \partial h^{(0)} / \partial v$, which is $\left.O\left(\epsilon^{1 / 2}\right)\right]$, but is much smaller, of order $\epsilon$, in the untrapped region. The consistency of this assumption can be verified after the solution is obtained. By making use of Eq. (1.19) for $C_{e}$ and expressing the velocity integral in Eq. (6.14) as a symmetrical quadratic form, we find that

$$
\left[Q_{11}^{(e)}\right]=-\int_{-\pi}^{\pi} \frac{d \theta}{2 \pi} \int d^{3} v \hat{h}^{(0)} C_{e}^{(0)} h^{(0)}+O(\epsilon),
$$

where the first term on the right-hand side is of order $\epsilon^{1 / 2}$, and is the dominant term, containing the pitchangle scattering part of $C_{e}$ :

$$
C_{e}^{(0)} \equiv\left(\nu_{e e}(v)+\nu_{e i}(v)\right) \mathcal{L} .
$$

The electron-ion term $\nu_{e i} \mathcal{L}$ was defined by Eqs. (5.3) and (5.5); the electron-electron collision frequency for pitch-angle scattering is defined by

$$
\nu_{e e}(v)=\frac{3 \pi^{1 / 2}}{4 \tau_{e}}\left(\frac{v_{\text {the }}}{v}\right)^{3} \phi\left(\frac{v}{v_{\text {the }}}\right),
$$

where

$$
\phi(x) \equiv\left(1-\frac{1}{2 x^{2}}\right) \operatorname{erf}(x)+\frac{e^{\omega x^{2}}}{\pi^{1 / 2} x},
$$

and $v_{\text {the }} \equiv\left(2 T_{e} / m_{e}\right)^{1 / 2}$. The $O(\epsilon)$ correction in Eq. (6.18) contains $G^{(1)}+\cdots$ as well as $C_{e}-C^{(0)}$; it will not be considered here.

By using Eq. (6.17) and the properties of $G^{(0)}$ (that it is independent of $\theta$ and even in $\sigma$ in the trapped region), we may verify that minimizing the zeroth order part of $\left[Q_{11}^{(e)}\right]$, Eq. (6.18) yields the zeroth-order constraint equation,

$$
\oint \frac{d \theta B}{v_{\|}} C_{e}^{(0)}\left(\frac{v_{\|} h}{\left|\Omega_{e p}\right|} f_{e 0}+G^{(0)}\right)=0 .
$$

By using this equation, Eq. (6.17) and the above-mentioned properties of $G^{(0)}$, the minimum value of $\left[Q_{11}^{(e)}\right]$ may be written as

$$
\begin{aligned}
Q_{11}^{(e)} & =-\int_{-\pi}^{\pi} \frac{d \theta}{2 \pi} \int d^{3} v \frac{v_{\|} h}{\left|\Omega_{e p}\right|} C_{e}^{(0)}\left(\frac{v_{\|} h}{\left|\Omega_{e p}\right|} f_{e 0}+G^{(0)}\right) \\
& =-\left(\alpha_{1}, g_{1 e}\right),
\end{aligned}
$$

with an error which is $O(\epsilon)$. This expression may be called a "variational expression" for the diffusion coef ficient, $-\left(\alpha_{1}, g_{1 e}\right)$, because of its derivation from the functional $\left[Q_{11}^{(e)}\right]$.

\section{Effects of electron-electron collisions}

The diffusion coefficient is to be calculated using the solution of Eq. (6.22); we note that this equation is the same as that solved in the Lorentz-model case, (except that the factor $\nu_{e i}$ is replaced by $\left.\nu_{e i}+\nu_{e e}\right)$. The solution is therefore given by Eq. (5.116). In the expression for the inner product, Eq. (5.120) we need only to replace $\nu_{e i}$ by $\nu_{e i}+\nu_{e e}$, in order to agree with Eq. (6.23). The integral over $v$ can be carried out analytically and we find the diffusion coefficient to be

$$
-\left(\alpha_{1}, g_{1 e}\right)=\frac{3}{8} n_{e} \frac{\rho_{e \theta}^{2}}{\tau_{e}}\left(1+\frac{0.53}{Z_{i}}\right) I,
$$

where $\rho_{e p}$, defined by Eq. (5.122), is now called $\rho_{e \theta}$, and

$$
I=1.38(2 \epsilon)^{1 / 2} \text {, }
$$

which is the large aspect-ratio limit of $I_{11}$, defined by Eq. (5.123).

By comparison with Eq. (5.121), the effect of electron-electron collisions is seen to be an increase, in the value of the diffusion coefficient, by a numerical factor of 1.53 for $Z_{i}=1,1.26$ for $Z_{i}=2$, etc. This may be surprising, in view of the fact that neoclassical diffusion, like classical diffusion, is due to unlike-species collisions. In the classical case, the form of the dis tribution function, which enters the integral for the (perpendicular) friction force, is not affected by like-species collisions. In the neoclassical case, on the other hand, the distribution function which goes into the calculation of the (parallel) friction force is quantitatively affected by electron-electron collisions, even though it is of zeroth order in the collision frequency.

In order to demonstrate this, we return to Eq. (5.119), and try to discover the distribution function $g_{1 e}$ which must be used in this expression. [Note that Eq. (6.23) contains the pitch-angle scattering operator $C_{e}^{(0)}$, rather than the exact $C_{e}$, which appears in Eq. (5.119).] A better approximation to the function $G$ is needed, so we return to the exact constraint equation, Eq. (5.106), and subtract from it the zeroth-order constraint equation, Eq. (6.22). To first order in the expansion of Eq. (6.16), we obtain

$$
\oint \frac{d \theta B}{v_{\mathrm{ll}}}\left(C_{e} G^{(1)}+C_{e}^{(1)} h^{(0)}\right)=0,
$$

where $C_{e}^{(1)} \equiv C_{e}-C_{e}^{(0)}$. We define a function $f_{* e}$ as the solution of

$$
C_{e} f_{* e}=-C_{e}^{(1)} h^{(0)} \text {. }
$$

Then the first-order constraint equation becomes

$$
\oint \frac{d \theta B}{v_{11}} C_{e} h^{(1)}=0
$$

where $h^{(1)} \equiv G^{(1)}-f_{* e}$, which can be assumed to be "localized" in the same sense as $h^{(0)}$, and can be explicitly solved for, in terms of $f_{* e}$. The function $f_{* e}$ is $O\left(\epsilon^{1 / 2}\right)$, like $h^{(0)}$, but is not "localized." These functions are shown in Fig. 7.

Returning to Eq. (6.23), we may write

$$
C_{e}^{(0)} h^{(0)}=C_{e} h^{(0)}-C_{e}^{(1)} h^{(0)}=C_{e}\left(h^{(0)}+f_{* e}\right),
$$

so that Eq. (6.23) becomes the same as Eq. (5.119) provided that, in the latter, we use the approximation

$$
g_{1 e} \simeq h^{(0)}+f_{* e} \text {. }
$$

The "direct" calculation of Eq. (5.119) therefore requires that the nonlocalized funtion $f_{* e}$ be included, while the "variational" calculation of Eq. (6.23) does not require it.

The function $f_{* e}$ contains the quantitative effects of electron-electron collisions. This is clear because Eq. (6.26) may be thought of as a generalized Spitzer problem, Eq. (5.44) with the right-hand side replaced by a more complicated function of velocity. As with the Spitzer distribution, the stronger is the effect of electronelectron collisions, relative to electron-ion collisions (measured by $1 / Z_{i}$ ), the less distorted is the distribution 


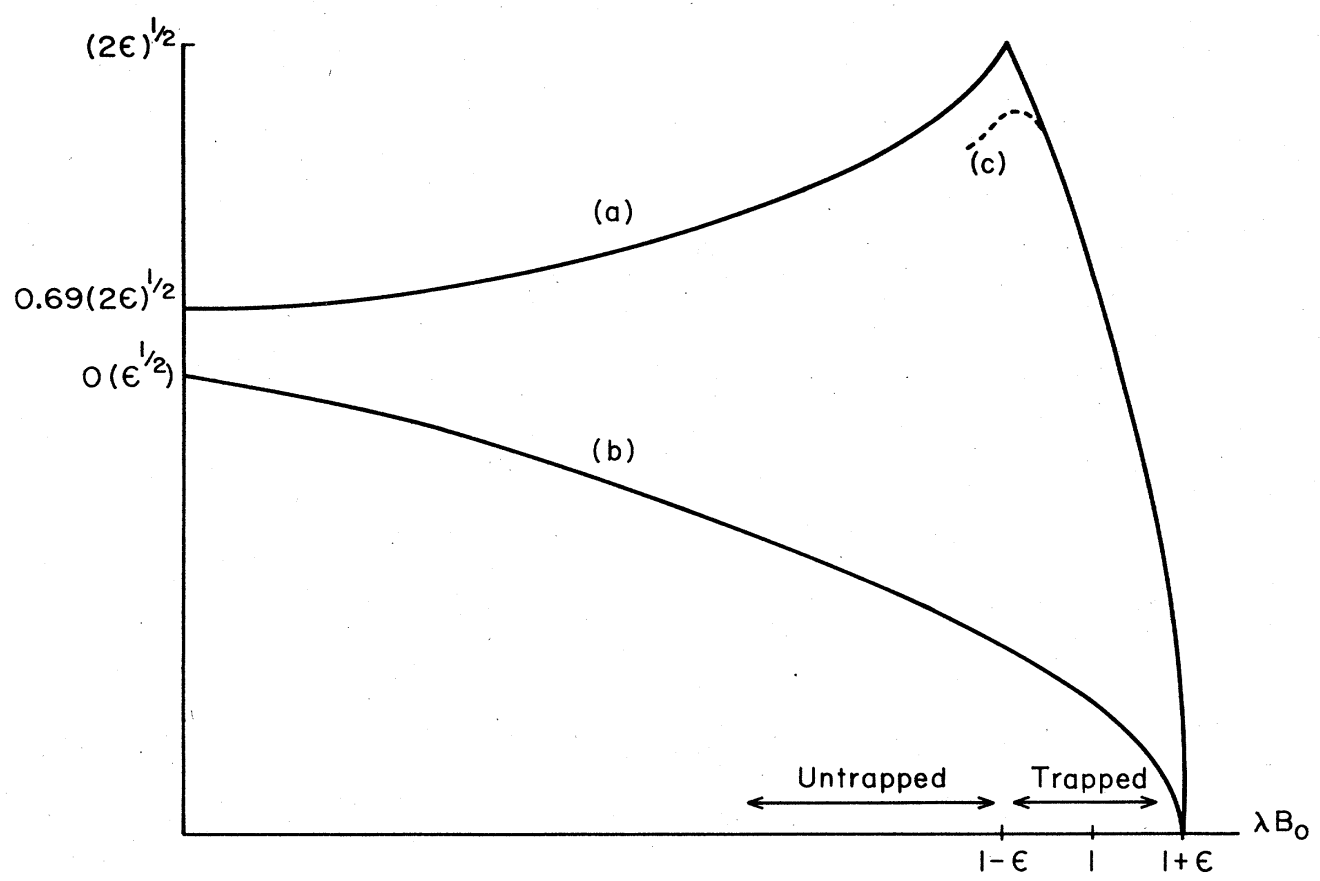

FIG. 7. Banana regime distribution functions: (a) the "localized" function $h^{(0)}$ [divided by $v f_{e 0} /\left|\Omega_{e p}\right|$ ] vs $\lambda B_{0}$, for $\epsilon=0.1$, and (b) the function $f_{* e}$, defined by Eq. (6.26) [divided by the same factor]: Both are evaluated at $\theta=0$, with the $O(\epsilon)$ corrections ignored. Also shown is (c) the smoothing of the function $g_{1 e}^{-}\left(\simeq h^{(0)}\right)$ in the boundary layer, as described by the solution of Eqs. (6.90) and (6.91).

from a Maxwellian (centered at a nonzero mean velocity, of course). In order to clarify the meaning of the force which drives the term $f_{* e}$ in the distribution function, we multiply Eq. (6.26) by $m_{e} v_{11}$ and integrate over velocity space. We note that $C_{e}^{(1)}$ (and therefore $f_{* e}$ ) is due to electron-electron collisions only: $C_{e}^{(1)}=C_{e e}-C_{e e}^{(0)}$. Since $C_{e e}$ conserves momentum, we have

$$
m_{e} \int d^{3} v v_{\|} C_{e i} f_{* e}-m_{e} \int d^{3} v v_{\|} C_{e e}^{(0)} h^{(0)}=0 .
$$

The second term may be called the friction force on the untrapped electrons, due to collisions with the trapped ones; the equation states that this must be balanced by the untrapped electron friction with the ions, calculated from $f_{* e^{\circ}}$ The term $f_{* e}$ is therefore driven by the friction between trapped and untrapped electrons, and this friction is the origin of the electron-electron contribution to the diffusion coefficient, Eq. (6.24). Such a contribution was first pointed out by Galeev and Sagdeev (1968).

The other transport coefficients may be calculated in a manner similar to that used for $\left(\alpha_{1}, g_{1 e}\right)$. Again, the electron-electron collisions make a contribution to the numerical coefficients; the results obtained by Rosenbluth, Hazeltine and Hinton (1972) may be written as follows

$$
\begin{aligned}
\left(\alpha_{1}, g_{1 e}\right) & =\left(\alpha_{1}, g_{4 e}\right)=\left(\alpha_{4}, g_{4 e}\right) \\
& =-0.73\left(1+0.53 / Z_{i}\right) \epsilon^{1 / 2} n_{e} \rho_{e \theta}^{2} / \tau_{e} \\
\left(\alpha_{1}, g_{2 e}\right) & =\left(\alpha_{2}, g_{4 e}\right) \\
& =1.10\left(1+0.41 / Z_{i}\right) \epsilon^{1 / 2} n_{e} \rho_{e \theta}^{2} / \tau_{e} \\
\left(\alpha_{2}, g_{2 e}\right) & =-2.37\left(1+0.43 / Z_{i}\right) \epsilon^{1 / 2} n_{e} \rho_{e \theta}^{2} / \tau_{e}
\end{aligned}
$$

$$
\begin{aligned}
\left(\alpha_{1}, g_{3 e}\right) & =\left(\alpha_{3}, g_{4 e}\right) \\
& =-1.46\left(1+0.67 / Z_{i}\right) \epsilon^{1 / 2} n_{e} c / B_{\mathrm{po}}, \\
\left(\alpha_{2}, g_{3 e}\right) & =\left(1.75 / Z_{i}\right) \epsilon^{1 / 2} n_{e} c / B_{\mathrm{po}}, \\
\left(\alpha_{3}, g_{3 e}\right) & =-1.46\left(1+0.34 / Z_{i}\right) \epsilon^{1 / 2} \sigma_{\|} / T_{e^{\bullet}}
\end{aligned}
$$

In Eqs. (6.31)-(6.33) the $Z_{i}$ dependence is an approximate fit, using the $Z_{i}=1$ and $Z_{i} \rightarrow \infty$ results. For $Z_{i}=1$, the numerical results of Spitzer and Harm (1953) have been used to evaluate the integrals. The conductivity $\sigma_{\| 1}\left(Z_{i}\right)$ is given by Eq. (5.66).

These results were generalized by Glasser and Thompson (1973) to arbitrary (noncircular) axisymmetric toroidal systems. The large aspect-ratio limit was used, in the form of the assumption of a small fraction of trapped particles. The above results, in which the approximation of Eq. (5.131) was made, may be so generalized as follows. The coefficients $\left(\alpha_{1}, g_{1 e}\right),\left(\alpha_{1}, g_{2 e}\right)$ and $\left(\alpha_{2}, g_{2 e}\right)$ should be multiplied by $I_{11} / 1.38(2 \epsilon)^{1 / 2} ;\left(\alpha_{1}, g_{3 e}\right),\left(\alpha_{2}, g_{3 e}\right)$, $\left(\alpha_{1}, g_{4 e}\right)$, and $\left(\alpha_{2}, g_{4 e}\right)$ should be multiplied by $I_{13} / 1.38$ $(2 \epsilon)^{1 / 2} ;\left(\alpha_{3}, g_{3 e}\right),\left(\alpha_{3}, g_{4 e}\right)$ and $\left(\alpha_{4}, g_{4 e}\right)$ should be multi plied by $I_{33} / 1.38(2 \epsilon)^{1 / 2}$; here $I_{11}, I_{13}$, and $I_{33}$ are given by Eqs. (5.123), (5.129), and (5.130).

The interpretation of these results is that the effective collision frequency has a contribution from collisions between trapped and untrapped electrons, as well as from collisions of trapped electrons with ions. The coefficient $\left(\alpha_{1}, g_{3 e}\right)$, which determines the trapped particle pinch effect and bootstrap current, increases when electronelectron collisions are included. This makes it clear that these effects are basically dependent for their exis tence upon collisions; although electron-ion collisions are sufficient, electron-electron collisions enhance the 
effects. In this connection, Kovrizhnykh (1970) has pointed out that the pinch effect coefficient for a weakly ionized plasma is smaller by roughly a factor $\epsilon$ than in a fully ionized plasma. This is due to the much stronger effect of velocity diffusion, in the latter.

The effective reduction in conductivity, given in Eq. (6.33), is also enhanced by electron-electron collisions. This is because the current-carrying untrapped electrons see the trapped electrons (in a time-average sense) as fixed scatterers, which are present in addition to the ion scatterers. The latter point has been discussed in a physical way by Coppi and Sigmar (1973).

One might be tempted to calculate the current density directly from the definition, Eq. (5.65), since the odd (in $\sigma)$ part of $H_{e}$ is presumably given by Eqs. (5.118) and (5.69) (Daybelge, 1971). This does not give the effect of electron-electron collisions correctly, however. It is necessary to include functions similar to $f_{* e}$, introduced above in connection with the calculation of the diffusion coefficient. This was first pointed out by Sigmar (1972), and was demonstrated in a way similar to the above analysis by Hazeltine, Hinton and Rosenbluth (1973). The point is that, since the functions analogous to $h^{(0)}$ and $f_{* e}$ (in the above analysis) are both $O\left(\epsilon^{1 / 2}\right)$ they must both be included in the "direct" calculation, Eq. (5.65). On the other hand, in the "variational" calculation [analogous to Eq. (6.23)] the $\lambda$ derivatives appear, and since $\partial f_{* e} / \partial \lambda$ is smaller than $\partial h^{(0)} / \partial \lambda$ by one order in $\epsilon^{1 / 2}, f_{* e}$ is not needed. The inclusion of the Spitzer function in the transformation Eq. (5.42), is necessary for obtaining this result, since the function $h^{(0)}$ would not otherwise have the property of "localization."

The electrical conductivity reduction coefficient, $\left(\alpha_{3}, g_{3 e}\right)$ has a numerical magnitude such that (for $Z_{i}=1$ ) the effective conductivity

$$
\sigma_{\text {eff }}=\sigma_{\text {II }}-T_{e}\left|\left(\alpha_{3}, g_{3 e}\right)\right| \text {, }
$$

unphysically changes sign for aspect ratios less than about 4 . In order to remedy this, the $O(\epsilon)$ correction to $\left(\alpha_{3}, g_{3 e}\right)$ was calculated by Hazeltine, Hinton, and Rosenbluth (1973). They retained the operator $C_{e}^{(1)}$ in Eq. (6.14), and also the function analogous to $f_{* e}$ for the conductivity problem; its contribution to the transport coefficient was calculated by using the variational principle for Eq. (6.26). The final result obtained is (for $Z_{i}=1$ )

$$
\sigma_{\text {eff }}=\sigma_{\mathrm{IL}}\left[1-1.95 \epsilon^{1 / 2}+0.95 \epsilon\right] \text {. }
$$

In the limit $\epsilon-1$, all of the electrons are trapped, and the effective electrical conductivity must go to zero. Equation (6.35) agrees with this fact, even though the small $\epsilon$ expansion should not be valid for $\epsilon \sim 1$. [Note that, in the Lorentz-gas approximation, $Z_{i} \gg 1$, the first correction to Eq. (6.34) is $O\left(\epsilon^{3 / 2}\right)$, and comes from a more exact evaluation of $I_{11}$ than is given by Eq. (6.25).]

The effective electrical conductivity was also calculated by Connor, Grimm, Hastie and Keeping (1973), using a model like-particle collision operator. A large aspect ratio expansion was not used; they obtained an analytical result which is valid for finite $\epsilon$. The accuracy of their result was limited by the use of the model operator how ever: the Spitzer conductivity, for example, was only reproduced to within $20 \%$, for $Z_{i}=1$.

\section{Ion transport in the banana regime}

\section{Ion thermal conductivity}

When the ions are in the banana regime, the calculation of the ion thermal conductivity $-\left(\beta_{2}, g_{2 i}\right)$ proceeds in a way very similar to the calculations in the previous subsection. The main difference is that the operator $C_{i i}$ conserves momentum, while $C_{e}$ does not. The consequences of this fact will become clear subsequently.

The banana regime variational expression is given by

$$
\left[Q_{22}^{(i)}\right]=-\left\langle\int d^{3} v \hat{g}_{2 i} C_{i i} g_{2 i}\right\rangle
$$

where

$$
g_{2 i}=-\left(v_{\|} h / \Omega_{i p}\right)\left(v^{2} / v_{\text {th } i}^{2}-5 / 2\right) f_{i_{0}}+G_{2 i},
$$

with $G_{2 i}$ independent of $\theta$, and even (in $\sigma$ ) in the trapped particle region. We use an expansion $G_{2 i}=G^{(0)}+G^{(1)}$ $+\cdots$, similar to Eq. (6.16), and further assume

$$
G^{(0)}=\left(v_{\|} h / \Omega_{i p}\right)\left(v^{2} / v_{\text {thi }}^{2}-y\right) f_{i_{0}}+h_{i}^{(0)},
$$

where $h_{i}^{(0)}$ is "localized" in the same sense as was $h_{1 e}^{(0)}$, and $y$ is a parameter to be determined. The lowestorder variational expression is similar to Eq. (6.18)

$$
\left[Q_{22}^{(i)}\right]=-\int_{-\pi}^{\pi} \frac{d \theta}{2 \pi} \int d^{3} v \hat{h}_{i}^{(0)} C_{i i}^{(0)} h_{i}^{(0)}
$$

The zeroth-order collision operator is

$$
C_{i i}^{(0)}=\nu_{i i}(v) \mathcal{L},
$$

with the pitch-angle scattering operator $\mathcal{L}$ given by Eq. (5.6), and

$$
\nu_{i i}(v)=\frac{3}{4} \frac{(2 \pi)^{1 / 2}}{\tau_{i}}\left(\frac{v_{\text {th } i}}{v}\right)^{3} \phi\left(\frac{v}{v_{\text {th } i}}\right),
$$

where $v_{\text {th } i} \equiv\left(2 T_{i} / m_{i}\right)^{1 / 2}, \phi(x)$ is given by Eq. (6.21), and

$$
\frac{1}{\tau_{i}} \equiv \frac{4}{3} \pi^{1 / 2} \frac{n_{i} Z_{i}^{4} e^{4} \ln \Lambda}{m_{i}^{1 / 2} T_{i}^{3 / 2}} .
$$

The zeroth-order constraint equation, obtained by requiring that $\left[Q_{22}^{(i)}\right]$ be minimized, is

$$
\oint \frac{d \theta B}{v_{\|}} C_{i i}^{(0)} h_{i}^{(0)}=0 \text {. }
$$

By using this equation, Eq. (6.37) and the properties of $G_{2 i}$, we may write the minimum value as

$$
\boldsymbol{Q}_{22}^{(i)}=\left\langle\int d^{3} v\left(v_{\|} h / \Omega_{i p}\right)\left(v^{2} / v_{\text {th } i}-y\right) C_{i i}^{(0)} h_{i}^{(o)}\right\rangle=-\left(\beta_{2}, g_{2 i}\right)
$$

which is the "variational" expression for the ion thermal conductivity.

The solution of Eq. (6.43) is

$$
\frac{\partial h_{i}^{(0)}}{\partial \lambda}=\frac{v}{2 \Omega_{i p}}\left(v^{2} / v_{\text {thi }}^{2}-y\right) f_{i_{0}}\left[\frac{1}{\xi}-\frac{H\left(h_{\min }-\lambda B_{0}\right)}{\langle\xi\rangle}\right],
$$

which may be substituted into Eq. (6.44).

Because of momentum conservation in $C_{i i}$, the term proportional to $y$ in Eq. (6.38) does not contribute to the integral in Eq. (6.36), but determines how much of $G^{(0)}$ is "localized." By considering $G^{(0)}$ to be a variational trial function, with $y$ as a parameter, we may calculate 
$y$ by minimizing $Q_{22}^{(i)}$, which is quadratic in $y$, as given by Eq. (6.44) with Eq. (6.45). The value of $y$ is then found to be determined by

$$
\int d^{3} v v_{\|} C_{i i}^{(0)} h_{i}^{(0)}=0 \text {. }
$$

This is equivalent to choosing $y$ consistent with momentum conservation (not automatically satisfied by the pitch-angle scattering approximation $\left.C_{i i}^{(0)}\right)$. The numerical value of $y$ so obtained, and the minimum value of $Q_{22}^{(i)}$ are

$$
y=1.33,-\left(\beta_{2}, g_{2 i}\right)=0.66 n_{i} \epsilon^{1 / 2} \rho_{i \theta}^{2} / \tau_{i} .
$$

These same results can be obtained in a more straightforward way by replacing $C_{i}$ by a model collision operator, suggested by Kovrizhnykh (1970), which consists of the pitch-angle scattering term $C_{i i}^{(0)}$ plus an $a d$ hoc momentum-conserving term. The details may be found in Appendix A of the paper by Rosenbluth, Hazeltine and Hinton (1972). The Kovrizhnykh model operator can be used to obtain the correct result, while other similar operators can not [see, e.g., Furth, et al., (1970)] because it is self-adjoint, and therefore leads to a variational principle, which is equivalent to Eq. (6.39).

The ion thermal conductivity, as given by Eq. (6.47), is the result of pitch-angle scattering of trapped ions by collisions with untrapped ones. [Recall, however, that the marginally trapped particles make the dominant contribution to the transport.] The outwardly diffusing ions are more energetic than the inwardly diffusing ones, resulting in a net energy flux outward. The constraint of zero net ion flux due to ion-ion collisions alone, expressed in terms of momentum conservation by Eq. (6.46), affects the magnitude of the energy flux, through the parameter $y$. The friction force of reaction on the untrapped ions, due to collisions with the trapped ones, produces a parallel flow, related to $y$ in a way described in the following subsection.

\section{Ion parallel flow}

The term proportional to $y$ in Eq. (6.38) represents a mean ion velocity parallel to the magnetic field, which is related to the parameter $\mu_{i}$ defined by Eq. (5.46). Using the weak-coupling approximation, we have $\mu_{i}$ $\simeq\left(\beta_{1}, g_{2 i}\right) A_{2 i}$, where $A_{2 i} \equiv(\partial / \partial \rho) \ln T_{i}$, and where the inner product can be calculated directly from the definition

$$
\left(\beta_{1}, g_{2 i}\right) \equiv\left[2 \Omega_{i_{p}} / n_{i_{0}} v_{\mathrm{th} i}^{2}\left\langle h^{-2}\right\rangle\right]\left\langle\frac{1}{h} \int d^{3} v v_{\|} g_{2 i}\right\rangle .
$$

Using Eqs. (6.37) and (6.38), and neglecting terms of order $\epsilon^{1 / 2}$, we find

$$
\left(\beta_{1}, g_{2 i}\right) \simeq 5 / 2-y,
$$

so that

$$
\mu_{i} \simeq(5 / 2-y) \frac{\partial}{\partial \rho} \ln T_{i} .
$$

The ion poloidal velocity, obtained by combining Eqs. (2.61) and (5.46); is proportional to $\mu_{i}$

$$
\overrightarrow{\mathrm{u}}_{i} \cdot \overrightarrow{\mathrm{B}}_{p} / B_{p}=\mu_{i} v_{\mathrm{thi}}^{2} / 2 \Omega_{i_{0}},
$$

where $\Omega_{i_{0}} \equiv Z_{i} e B_{0} / m_{i} c$. This poloidal rotation is a result of collisions between trapped and untrapped ions, in the presence of a temperature gradient.

The ion parallel flow velocity represents an additional source of friction on the electrons: the fourth electron thermodynamic force is given by

$$
A_{4 e} \equiv-\left(T_{i} / Z_{i} T_{e}\right) \mu_{i} \simeq(y-5 / 2)\left(T_{i} / Z_{i} T_{e}\right) \frac{\partial}{\partial \rho} \ln T_{i} .
$$

The $O\left(\epsilon^{1 / 2}\right)$ correction may be neglected, since $A_{4 e}$ is multiplied, in Eqs. (5.70)-(5.72), by transport coefficients which are themselves of order $\epsilon^{1 / 2}$. This force can be combined with $A_{1 e}$, in the large aspect-ratio limit because of the fact that $\alpha_{4}$ differs from $\alpha_{1}$ only by terms of order $\epsilon$. Hence,

$$
\left(\alpha_{4}, g_{n e}\right) \simeq\left(\alpha_{1}, g_{n e}\right)
$$

and the Onsager relations, Eq. (5.98) imply further that

$$
\left(\alpha_{n}, g_{4 e}\right) \simeq\left(\alpha_{n}, g_{1 e}\right) \text {. }
$$

In Eqs. (5.70)-(5.72), therefore, $A_{4 e}$ is multiplied by the same factor as $A_{1 e}$, and we may use the combined force

$$
\begin{aligned}
A_{1 e}+A_{4 e}= & \left(\frac{\partial}{\partial \rho} \ln n_{e}\right)\left(1+T_{i} / Z_{i} T_{e}\right)+\frac{\partial}{\partial \rho} \ln T_{e} \\
& +\left(T_{i} / Z_{i} T_{e}\right)(y-3 / 2) \frac{\partial}{\partial \rho} \ln T_{i},
\end{aligned}
$$

and thus reduce the number of independent electron forces from four to three.

Again, using Eq. (6.52), the ion force $A_{1 i}$, given by Eq. (5.75), may be written as

$$
A_{1 i} \simeq\left(1 / Z_{i}\right) \sum_{n=1}^{4}\left(\alpha_{1}, g_{n e}\right) A_{n e} \equiv \Gamma_{e} / Z_{i}
$$

The $O\left(m_{e} / m_{i}\right)^{1 / 2}$ correction to the ion heat flux is therefore

$$
q_{i}-T_{i}\left(\beta_{2}, g_{2 i}\right) A_{2 i} \simeq T_{i}\left(\beta_{2}, g_{1 i}\right) \Gamma_{e} / Z_{i} .
$$

Using the Onsager relation $\left(\beta_{2}, g_{1 i}\right)=-\left(\beta_{1}, g_{2 i}\right)$ and Eq. (6.49), we have, finally

$$
q_{i}-T_{i}\left(\beta_{2}, g_{2 i}\right) A_{2 i} \simeq(y-5 / 2) T_{i} \Gamma_{e} / Z_{i} .
$$

Thus, the effect of electron friction on the ions results in an additional ion heat flux, which is proportional to the ion flux $\Gamma_{i}\left(=\Gamma_{e} / Z_{i}\right)$.

In addition to the term $\mu_{i}$ in the ion parallel velocity, Eq. (5.46), there are also terms proportional to the ion pressure gradient and the radial electric field. We shall give an interpretation of these terms, in terms of guiding-center orbits. Note that the ion parallel velocity is approximately equal to the toroidal flow velocity of the plasma, for large aspect ratio.

We first point out that the pressure gradient and radial electric field terms in Eq. (5.46) are easily understood in terms of the trapped ion banana orbits. We may write $\partial \ln p_{i} / \partial \rho=\partial \ln n_{i} / \partial \rho+\partial \ln T_{i} / \partial \rho$, and interpret the density and temperature gradient terms separately. A net flow of trapped ions, along field lines, occurs when there is a density gradient. This is because of the noncancella- 
tion of the numbers of ions at a given value of $r$, with values of $v_{\|}$which are equal in magnitude but opposite in sign, since these come from different average radial locations, and hence from regions of different density. A similar effect occurs for the temperature contribution to the pressure gradient, where particles moving in one direction along $\vec{B}$ are more energetic than those moving in the opposite direction. The radial electric field affects a single trapped ion by giving it a greater kinetic energy on one half of its orbit than on the other half, these being at different values of $r$, and hence at different values of electrostatic potential energy. The untrapped ion contributions to Eq. (5.46) can not be so simply explained, however, and we must now consider collisions between the trapped and untrapped ions.

Consider an initial state of the plasma, with no net angular momentum, and no radial electric field. For simplicity, assume also that there is no ion temperature gradient. In Eq. (5.46), choose $\mu_{i}=\partial \ln p_{i} / \partial \rho$ initially; the toroidal mass flow velocity is then proportional to $\epsilon \cos \theta$, and is thus in opposite directions in opposite halves of the plasma cross section. Next, consider the flow produced by the ion orbits, in the absence of collisions. The orbits of the untrapped ions, and the density gradient, produce a flow similar to that in the initial state. The trapped ions have a parallel flow velocity which is unidirectional, but which is very small (of order $\epsilon^{3 / 2}$ ) for large aspect ratio. The distribution function would then be discontinuous at the boundary between the trapped and untrapped ions. Even a small rate of ion-ion collisions implies a rapid smoothing of this discontinuity, which leads to a unidirectional flow of untrapped ions. The resulting quasi-equilibrium distribution is given by the first two terms in Eq. (5.43); the flow velocity is given by Eq. (5.46) with $\mu_{i}=0$.

The relaxation of the distribution function can be explained in terms of the decay of poloidal rotation. Since the trapped ions have no net poloidal velocity, the effect of collisions with the untrapped ions is to reduce the poloidal velocity of the latter to zero also. The rate of decay of poloidal velocity by this process, was shown by Rosenbluth (1973) to be of order $\tau_{i}^{-1}$ [given by Eq. (6.42)].

The unidirectional plasma flow, given by Eq. (5.46), would represent a large net toroidal angular momentum, if the radial electric field were zero. Since angular momentum is conserved, there is an apparent paradox, which was discussed by Sagdeev and Galeev (1970). Actually, the plasma angular momentum can change only if the electromagnetic field angular momentum changes, so that the sum is constant. A change in the plasma angular momentum thus implies a change in the radial electric field, and this affects the value of the toroidal flow velocity. The unidirectional flow is thereby held at a small value, as we shall now demonstrate.

By adding the toroidal components of the electron and ion momentum equations, Eq. (2.15), and using quasineutrality, we obtain an equation for the plasma toroidal angular momentum

$$
\frac{\partial}{\partial t}\left\langle m_{i} n_{i} u_{i T} R\right\rangle+\frac{1}{r} \frac{\partial}{\partial r} r S_{i}=\frac{1}{c}\langle\vec{J} \cdot \nabla \psi\rangle,
$$

where the stress is [c.f., Eq. (2.91)]

$$
S_{i} \equiv\left\langle R^{2} \nabla \zeta \cdot \mathrm{P}_{i} \cdot \nabla r\right\rangle .
$$

Thus, the toroidal angular momentum changes either by diffusion or because of the torque due to the $\vec{J} \times \vec{B}$ force, with the radial current flow. The relaxation of an arbitrary initial distribution, to that given by Eq. (5.43), occurs in a sufficiently short time (of order $\tau_{i}$ ) that the stresses may be neglected.

The relaxation of the distribution function results in a radial current flow. This can be understood from Fig. 8 , where we have plotted four typical untrapped ion orbits, labeled A, B, C, and D. The effect of friction with the trapped ions is to populate the D orbits at the expense of the A orbit population; the parallel flow velocity must have the same direction everywhere on a magnetic surface, which implies an inversion of the relative populations. Indicating the populations by $F(\mathrm{~A})$, etc., we then have

$$
F(\mathrm{~B})>F(\mathrm{D})>F(\mathrm{~A})>F(\mathrm{C}),
$$

assuming a monotonic decrease in density away from the magnetic axis, uniform temperature, and zero radial electric field. In addition to increasing the angular momentum, this population inversion causes a net radial movement of the average ion position, and hence a radial current.

This radial current implies the buildup of a negative radial electric field, which limits drastically the angular momentum buildup. We note that

$$
4 \pi\langle\overrightarrow{\mathrm{J}} \cdot \nabla \psi\rangle+\frac{\partial}{\partial t}\langle\overrightarrow{\mathrm{E}} \cdot \nabla \psi\rangle=c\langle\nabla \cdot(\overrightarrow{\mathrm{B}} \times \nabla \psi)\rangle=0,
$$

and substituting this into Eq. (6.58) gives

$$
\left\langle m_{i} n_{i} u_{i T} R\right\rangle+\langle\overrightarrow{\mathrm{E}} \cdot \nabla r\rangle R_{0} B_{\mathrm{po}} / 4 \pi c=\text { const. }
$$

This is the statement of conservation of total angular momentum in the plasma and the electromagnetic field. By assuming the constant to be zero (from initial conditions) and combining this equation with Eq. (5.46), using $u_{i T} \simeq u_{i \|}$, we may eliminate the radial electric field:

$$
\left\langle u_{i \|} h\right\rangle=\frac{v_{\mathrm{thi}}^{2}}{2 \Omega_{i p} \kappa}\left[\mu_{i}-\frac{\partial \ln p_{i}}{\partial \gamma}\right]+\boldsymbol{O}\left(\epsilon^{2}\right),
$$

where

$$
\kappa \equiv 1+4 \pi m_{i} n_{i} c^{2} / B_{\text {po }}^{2}
$$

is an effective dielectric constant. For typical tokamak parameters, $\kappa \gg 1$, so the actual buildup of plasma toroidal angular momentum by this process is negligible. A similar conclusion was obtained by Stix (1973).

On a longer time scale, of course, the stress term in Eq. (6.58) may not be neglected, and the diffusion of angular momentum between flux surfaces determines the toroidal flow velocity. [The radial current term in Eq. (6.58) must then be zero, assuming approximate plasma neutrality on the longer time scale.] The plasma can develop a net toroidal angular momentum by transferring angular momentum to the solid boundary which surrounds the plasma. The time dependence of the radial electric field is thus determined, through Eq. (5.46). [On this longer time scale, Eq. (6.62) no longer holds.]

The stress term has been calculated by Rosenbluth, Rutherford, Taylor, Frieman, and Kovrizhnykh (1971). 
In addition to a toroidal viscosity, relating the stress to the rate of strain $\partial u / \partial r$, they obtained a term which couples the rotation to the ion temperature gradient. Large values, for the rotation velocity and electrostatic potential, were implied by their results. However, the time necessary to establish such steady states was found to be very long, of order $\epsilon^{-3 / 2}$ times the ion heat conduction time.

The loss of ion toroidal momentum, due to charge-exchange reactions, was considered by Sigmar, Clarke, Neidigh, and Vander Sluis (1974). They found that a steady state is reached on a time of order $\nu_{\mathrm{cx}}^{-1}$ $\equiv\left[n_{0}\left\langle\sigma_{\mathrm{cx}} v\right\rangle\right]^{-1}$, where $\sigma_{\mathrm{cx}}$ is the charge-exchange cross section. The radial electric field is then determined, in terms of the density and temperature gradients.

The effect of nonaxisymmetric toroidal field ripples on plasma rotation has been considered by Rosenbluth (1973), Connor and Hastie (1973), and Tsang and Frieman (1975). They found a decay of toroidal rotation in a few ion collision times, even with very small ripples. A detailed discussion of this process, as well as the other processes determining toroidal rotation, is beyond the scope of the present review.

\section{The banana-plateau transition}

\section{The plateau limit}

The behavior of the transport coefficients, as functions of collision frequency, is of some interest, especially because the condition for the banana regime cannot be satisfied at all values of minor radius $r$, in a given plasma, even at high temperatures. At small values of $r / R_{0}$, we may approach the limiting case in which the diffusion coefficient, for example, becomes independent of collision frequency. This is known as the "plateau regime," and was discovered by Galeev and Sagdeev (1968).

From the qualitative description given in Sec. III.D, we expect that only a small ("resonant") range of particle velocities is involved, so that the Fokker-Planck collision operators may be simplified as in the banana regime. We assume that pitch-angle scattering dominates in the like-particle collision terms, as well as in the electron-ion collision term. This can be verified a posteriori for the appropriate range of particle energies, although the "plateau regime" approximations are not valid for all particle energies, because of the energy-dependence of the collision frequency.

\section{a. Electron transport}

We therefore begin with the electron kinetic equation, Eq. (5.80), in which the collision operator is to be replaced by $C_{e}^{(0)}$, given by Eq. (6.19). It is more convenient to use $(\theta, \xi)$ as independent variables, rather than $(\theta, \lambda)$, where $\xi \equiv(1-\lambda B)^{1 / 2}$. By neglecting some terms of order $\epsilon^{3}$, Eq. (5.80) becomes

$$
\begin{aligned}
v\left(B_{\mathrm{po}} / r B\right)\left[\xi \frac{\partial g_{n e}}{\partial \theta}-\frac{\epsilon}{2} \sin \theta\left(1-\xi^{2}\right) \frac{\partial g_{n e}}{\partial \xi}\right] \\
-\frac{1}{2}\left(\nu_{e e}+\nu_{e i}\right) \frac{\partial}{\partial \xi}\left(1-\xi^{2}\right) \frac{\partial g_{n e}}{\partial \xi}=-\alpha_{n} f_{e 0} .
\end{aligned}
$$

In this subsection, we shall consider only the $n=1$ equa-

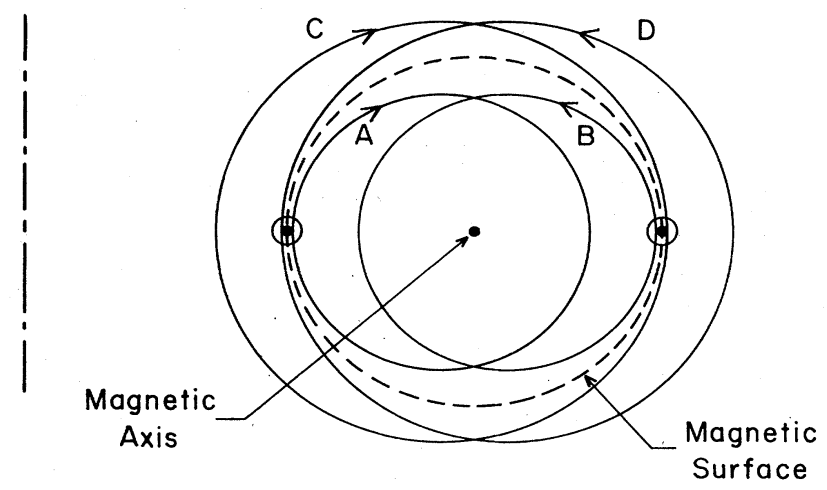

FIG. 8. Four typical untrapped ion orbits, illustrating the effects of collisions with the trapped ions: the parallel flow must be out of the paper at both circled points, which requires more particles in D orbits than in A orbits. The density decreases away from the magnetic axis and is constant on a magnetic surface, with the deficit in A orbits relative to $D$ orbits compensated by the greater number of $\mathrm{B}$ orbits relative to $\mathrm{C}$ orbits, as given by Eq. (6.59).

tion, in which the factor on the right-hand side is

$$
\alpha_{1}=\frac{\sin \theta}{\left|\Omega_{e}\right| R} \frac{v^{2}}{2}\left(1+\xi^{2}\right) .
$$

We define a dimensionless collision frequency (which is a function of velocity $v$ ) by

$$
\nu \equiv\left(\nu_{e e}+\nu_{e i}\right) r B / B_{\mathrm{po}} v .
$$

The plateau regime limit is defined by Eq. (3.52), or

$$
\epsilon^{3 / 2} \ll \nu \ll 1 \text {. }
$$

(Recall that the banana regime limit is $\nu \ll \epsilon^{3 / 2}$.) The assumption $\nu \ll 1$ is necessary to ensure that the solution of Eq. (6.63) is sufficiently well localized. In fact, we may estimate the localization width by comparing the first and third terms on the left-hand side: $\Delta \xi \sim \nu /(\Delta \xi)^{2}$, or $\Delta \xi \sim \nu^{1 / 3}$. We introduce a "boundary-layer variable", $p$, such that $p \sim 1$. when $\xi \sim \nu^{1 / 3}$

$$
p \equiv \nu^{-1 / 3} \xi \text {. }
$$

After neglecting terms of order $\nu^{2 / 3}$, the equation becomes

$$
\begin{aligned}
p \frac{\partial g_{1 e}}{\partial \theta}-\frac{1}{2}\left(\epsilon / \nu^{2 / 3}\right) & \sin \theta \frac{\partial g_{1 e}}{\partial p}-\frac{1}{2} \frac{\partial^{2} g_{1 e}}{\partial p^{2}} \\
= & -\frac{1}{2}\left(\epsilon / \nu^{1 / 3}\right)\left(v /\left|\Omega_{e \theta}\right|\right) \sin \theta f_{e 0} .
\end{aligned}
$$

An expansion in the small parameter $\epsilon / \nu^{2 / 3}$ yields

$$
g_{1 e}=-\frac{1}{2}\left(\epsilon / \nu^{1 / 3}\right)\left(v /\left|\Omega_{e p}\right|\right) f_{e 0} \operatorname{Im}\left(g_{0} e^{i \theta}\right),
$$

where $g_{0}$ is a solution of

$$
\frac{1}{2}\left(d^{2} g_{0} / d p^{2}\right)-i p g_{0}=-1 \text {. }
$$

By extending the interval, in which $g_{0}$ is defined, from $-\nu^{-1 / 3}<p<\nu^{-1 / 3}$ to $-\infty<p<\infty$, we may solve this equation by Fourier transformation. The solution which tends to zero for $|p| \rightarrow \infty$ is (Su and Oberman, 1968)

$$
g_{0}=\int_{0}^{\infty} d t \exp \left[-i p t-t^{3} / 6\right] \text {. }
$$


It is easily verified that $g_{0} \sim 1 / p$, for $p \gg 1$, so that $g_{1 e}$ is indeed localized to a range of approximately $\Delta p \sim 1$, or $\Delta \xi \sim \nu^{1 / 3}$. Although this range varies with $v$ approximately as $v^{-4 / 3}$, the coordinate perpendicular to the "boundary layer" is approximately the pitch-angle coordinate $\xi$, for small $\nu$, and this justifies the pitch-angle scattering approximation.

In order to calculate the "plateau regime" diffusion coefficient, we must assume that all particle energies, which contribute to the velocity integral in Eq. (5.74), satisfy the plateau regime condition, Eq. (6.66). By using the approximation

$$
\nu^{-1 / 3} \int_{-1}^{1} d \xi \operatorname{Re} g_{0} \simeq \int_{-\infty}^{\infty} d p \operatorname{Re} g_{0}=\pi,
$$

we obtain

$$
\left(\alpha_{1}, g_{1 e}\right) \simeq-\left(\pi^{1 / 2} / 4\right) \epsilon^{2} n_{e} \rho_{e \theta}^{2}\left(B_{\mathrm{po}} v_{\text {the }} / r B\right),
$$

which is independent of collision frequency (and hence independent of $Z_{i}$ ). This result was first obtained by Galeev and Sagdeev (1968), by using a Krook model collision operator. [The numerical value is correctly given in the paper by Galeev (1971).] The above derivation, using the pitch-angle scattering model, was first given by Rutherford (1970).

The other electron transport coefficients may be obtained in a very similar way; the results are

$$
\begin{aligned}
& \left(\alpha_{1}, g_{2 e}\right)=-\frac{\pi^{1 / 2}}{8} \epsilon^{2} n_{e} \rho_{e \theta}^{2}\left(v_{\text {the }} B_{\mathrm{po}} / r B\right) \\
& \left(\alpha_{2}, g_{2 e}\right)=-\frac{13}{16} \pi^{1 / 2} \epsilon^{2} n_{e} \rho_{e \theta}^{2}\left(v_{\text {the }} B_{\mathrm{po}} / r B\right) \\
& \left(\alpha_{1}, g_{3 e}\right)=-\left(0.26+0.24 / Z_{i}\right)^{-1} \epsilon^{2}\left(n_{e} c / B_{\mathrm{po}}\right)\left(v_{\text {the }} \tau_{e} B_{\mathrm{po}} / r B\right)
\end{aligned}
$$

$\left(\alpha_{2}, g_{3 e}\right)=-\left(0.13+0.27 / Z_{i}\right)^{-1} \epsilon^{2}\left(n_{e} c / B_{\mathrm{po}}\right)\left(v_{\text {the }} \tau_{e} B_{\mathrm{po}} / r B\right)$,

$\left(\alpha_{3}, g_{3 e}\right)=-\left(0.11+0.25 / Z_{i}\right)^{-1} \epsilon^{2}\left(v_{\text {the }} \tau_{e} B_{\mathrm{po}} / r B\right) \sigma_{\|} / T_{e}$,

where $\sigma_{\|}$is given by Eq. (5.66). [The inner products involving $\alpha_{4}$ or $g_{4 e}$ are obtained by using Eqs. (6.52) and (6.53), as in the banana regime.] In Eqs. (6.74)-(6.76), the $Z_{i}$ dependence is an approximate fit to the results for $Z_{i}=1$ and $Z_{i} \gg 1$. For $Z_{i} \gg 1$, Eq. (5.132) was used for $f_{s e}$, while the $Z_{i}=1$ values were calculated using the simple approximation

$$
f_{s e} \simeq-0.36\left(e / T_{e}\right) \tau_{e}\left[\left(v / v_{\text {the }}\right)^{2}+2 v / v_{\text {the }}\right] f_{e 0}, \text { for } Z_{i}=1 .
$$

The thermal diffusion coefficient, $\left(\alpha_{1}, g_{2 e}\right)$ and the thermal conductivity, $-\left(\alpha_{2}, g_{2 e}\right)$ are independent of collision frequency, like the particle diffusion coefficient, $-\left(\alpha_{1}, g_{1 e}\right)$. The trapped particle pinch effect coefficient $\left(\alpha_{1}, g_{3 e}\right)$, which is also the bootstrap current coefficient, decreases with increasing collision frequency, as do the thermal pinch coefficient $\left(\alpha_{2}, g_{3 e}\right)$ and the conductivity correction coefficient, $\left(\alpha_{3}, g_{3}\right)$. The latter three coefficients represent effects which only exist for sufficiently small collision frequencies, when guiding-center orbit effects dominate, so it is natural that they should approach zero at higher collision frequencies.

\section{b. Ion transport}

In solving the ion-kinetic equation, Eq. (5.81), we must allow for the fact that the operator $C_{i i}$ conserves momentum. Thus, when Eq. (5.81) (for $n=2)$ is written in a form analogous to Eq. (6.63), the mirror force term (proportional to $\epsilon \sin \theta$ ) plays a significant role. Using the variables $(\theta, \xi)$, we have

$$
\begin{aligned}
\xi \frac{\partial g_{2 i}}{\partial \theta}-\left(r B / B_{\mathrm{po}} v\right) C_{i i} g_{2 i} & =\frac{1}{2} \epsilon \sin \theta\left(1-\xi^{2}\right) \frac{\partial g_{2 i}}{\partial \xi} \\
& -\left(r B / B_{\mathrm{po}} v\right) \beta_{2} f_{i_{0}}
\end{aligned}
$$

where

$$
\beta_{2}=\left(-v^{2} / v_{\text {thi }}^{2}+5 / 2\right) \frac{\sin \theta}{\Omega_{i} R} \frac{v^{2}}{2}\left(1+\xi^{2}\right) .
$$

Recalling that the $\theta$ derivative is at constant $\xi$, we note that a term $\left(v \xi u_{\|} / v_{\text {thi }}^{2}\right) f_{i_{0}}$ may be added to the function $g_{2 i}$, without contributing to the left-hand side of Eq. (6.78), provided that $\partial u_{\|} / \partial \theta=0$. In order that this equation have a solution at all, the right-hand side must be orthogonal to the above null solution. That is, multiplication of Eq. (6.78) by $v^{2} \xi$ and integration over $\theta$ and $\vec{v}$ yields the constraint

$$
\frac{1}{2} \epsilon \int_{-\pi}^{\pi} d \theta \sin \theta \int d^{3} v v^{2} \xi\left(1-\xi^{2}\right) \frac{\partial g_{2 i}}{\partial \xi}=0,
$$

which serves to determine the arbitrary parameter $u_{\|}$. This parameter can be introduced, in terms of the parameter $y$ just as in the banana regime [c.f. Eqs. (6.37) and (6.38)]:

$$
g_{2 i}=\left(\frac{5}{2}-y\right)\left(v_{\|} h / \Omega_{i p}\right) f_{i_{0}}+H_{i},
$$

where $H_{i}$ may be assumed to be localized. Substitution into Eq. (6.78) and use of Eq. (6.40) for the collision term gives

$$
\begin{aligned}
\xi \frac{\partial H_{i}}{\partial \theta}-\frac{1}{2} \epsilon \sin \theta & \left(1-\xi^{2}\right) \frac{\partial H_{i}}{\partial \xi}-\frac{1}{2} \nu \frac{\partial}{\partial \xi}\left(1-\xi^{2}\right) \frac{\partial H_{i}}{\partial \xi} \\
& =\left(v^{2} / v_{\mathrm{thi}}^{2}-y\right) \frac{\epsilon}{2} \sin \theta \frac{v\left(1+\xi^{2}\right)}{\Omega_{i p}} f_{i_{0}},
\end{aligned}
$$

where

$$
\nu \equiv \nu_{i i}(v) r B / B \quad v \text {. }
$$

Following the same method of analysis, as was used for Eq. (6.63), we find the approximate solution

$$
H_{i}=\frac{1}{2}\left(\epsilon / \nu^{1 / 3}\right)\left(v / \Omega_{i p}\right)\left(v^{2} / v_{\text {thi }}^{2}-y\right) f_{i_{0}} \operatorname{Im}\left(g_{0} e^{i \theta}\right),
$$

where $g_{0}$ is given by Eqs. (6.70), (6.67), and (6.83).

Now the ion thermal conductivity may be calculated

$$
-\left(\beta_{2}, g_{2 i}\right)=\int_{-\pi}^{\pi} \frac{d \theta}{2 \pi} \frac{\sin \theta}{\Omega_{i} R} \int d^{3} v \frac{v^{2}}{2}\left(v^{2} / v_{\mathrm{th} i}^{2}-5 / 2\right) H_{i},
$$

where the factor $1+\xi^{2}$ has been replaced by unity, since $H_{i}$ is localized to small values of $\xi$. The value of $y$, which helps determine the numerical value of the thermal conductivity, is determined by Eq. (6.80). After integration by parts, and replacing $1-3 \xi^{2}$ by unity, this becomes

$$
\int_{-\pi}^{\pi} d \theta \sin \theta \int d^{3} v v^{2} H_{i}=0
$$


which is equivalent to the condition of zero ion flux, when $1+\xi^{2}$ (in the expression for the drift velocity) is replaced by unity. Comparing this with Eq. (6.84), we see that the $\frac{5}{2}$ in the integrand can be omitted; alternatively, the $\frac{5}{2}$ can be replaced by $y$, making the ion thermal conductivity quadratic in $y$, as in the banana regime variational calculation. The condition which determines $y$, Eq. (6.85), is then equivalent to minimizing the thermal conductivity with respect to $y$. The results of carrying out the integrals in Eq. (6.84) and determining $y$ in this way, are

$$
y=3, \quad\left(\beta_{2}, g_{2 i}\right)=-\frac{3}{4} \pi^{1 / 2} \epsilon^{2} n_{i} \rho_{i \theta}^{2}\left(v_{\mathrm{th} i} B_{\mathrm{po}} / r B\right) .
$$

The thermal conductivity given by this expression is the result of scattering of "resonant" ions (in the sense described in Sec. III.D) by nonresonant ones. The frictional force of reaction, back on the nonresonant ions, produces a parallel flow related to $y$ in the same way as described in Sec. VI.C; Equations (6.49)-(6.51) of that section remain true in the plateau regime as well as the banana regime, with only the numerical values of $y$ being different in the two regimes. Likewise, Eqs. (6.54) and (6.57) hold also in the plateau regime.

\section{Variational calculation}

The collision-frequency dependence of the transport coefficients, in the entire transitional range of collision frequencies between the banana regime and plateau regime, is quite relevant to present experiments. This dependence was calculated by Hinton and Rosenbluth (1973). They carried out a numerical solution of Eqs. (6.63) and (6.82) by a finite difference relaxation method, which makes use of a maximal variational principle to gain rapid convergence. The transport coefficients were calculated from variational expressions, so that only the localized parts of the distribution functions were needed, and the pitch-angle scattering approximation was adequate. Good agreement was thus obtained with the banana regime limiting values which had been calculated analytically, as described in Secs. VI.B and C.

The starting point for the calculation of the electron transport coefficients, is Eq. (5.96). The functions $g_{n a}$ are assumed to be localized in the variable $\lambda$, so that the collision operator can be replaced by the pitch-angle scattering operator, Eq. (6.19). [This variational principle is then equivalent to Eq. (6.63), when the transformation to the variables $(\theta, \xi)$ is made.] Using the pitch-angle scattering approximation, it is possible to eliminate the two functions $g_{1 a}^{+}, g_{1 a}^{-}$(for $n=1$, for example) in favor of a single function $\Phi_{1 a}$. This function is like a "potential," in that both $g_{1 a}^{ \pm}$may be derived from it by differentiation.

The variational principle, when expressed in terms of this single function, is maximal. That is $V_{11}^{(a)}$ has an absolute maximum when the function $\Phi_{1 a}$ satisfies the appropriate partial differential equation and boundary conditions. This property was found to be quite useful, in combination with a finite-difference relaxation method of solving the partial differential equations on a computer. By choosing the relaxation parameter to maximize $V_{11}^{(a)}$ after each iteration, an increasing sequence of values was generated, which converged to the abso- lute maximum of $V_{11}^{(a)}$ at the maximum possible rate.

The value of $V_{11}^{(a)}$ so obtained (using the arbitrary convergence criterion that the fractional change in $V_{11}^{(a)}$, after one iteration, be less than $10^{-6}$ ) gave the numerical values of the transport coefficients. These were calculated for three inverse aspect ratios, $\epsilon=0.01,0.04$, and 0.09 , and for a range of values of collisionality, $10^{-2}<\nu_{* e}<10$, where the electron collisionality parameter is defined as

$$
\nu_{* e} \equiv \sqrt{2} r B_{0} /\left(B_{\mathrm{po}} v_{\text {the }} \tau_{e} \epsilon^{3 / 2}\right),
$$

(the banana-plateau transition occurs nominally for $\left.\nu_{* e} \sim 1\right)$. These results were then fitted to analytical formulas (which will be presented in Sec. VI.F) using the method of least squares.

The starting point for the ion thermal conductivity calculation, is a variational principle equivalent to Eq. (6.82), which may be obtained by substituting Eq. (6.81) into Eq. (5.96) for $m=n=2, c=i$, and using the pitchangle scattering operator, Eq. (6.40), on the localized function $H_{i}$. The numerical method of calculation, using the maximal variational principle, is the same as for the electron transport coefficients. The value of the parameter $y$ is obtained, as in the banana regime calculation, by minimizing the ion thermal conductivity, which is quadratic in $y$. Analytical formulas, obtained from least squares fits to $y$ and $\left(\beta_{2}, g_{2 i}\right)$, as functions of ion collisionality, will be presented in Sec. VI.F.

Analytical checks on the accuracy of the numerical calculations, were made in the following way. We define the functions $Q_{m n}(\nu)$ in terms of the variational expressions, Eq. (5.96), before integrating over particle energy; for example,

$$
\begin{aligned}
V_{11}^{(e)} & =-\left(\alpha_{1}, g_{1 e}\right), \\
& =2 \epsilon^{1 / 2}\left(B_{\mathrm{po}} / r B\right) \int_{0}^{\infty} v^{3} d v f_{e 0}(v)\left(v / \Omega_{e p}\right)^{2} \nu Q_{11}(\nu),
\end{aligned}
$$

where $\nu$ is defined as a function of $v$ by Eq. (6.65). (Since $v$ appears in Eqs. (6.63) and (6.82) only as a parameter, the matrix elements $Q_{m n}$ themselves provide variational principles for these equations.) In the banana regime limit, $\nu \ll \epsilon^{3 / 2}, Q_{11}(\nu)$ must approach the constant value $(\pi / 2) I \epsilon^{-1 / 2}=3.07$ [where $I$ is given by Eq. (6.25)]. In the plateau regime limit, $\epsilon^{3 / 2} \ll \nu \ll 1$, the function $Q_{11}(\nu)$ is given approximately by

$$
\nu Q_{11}(\nu) \simeq\left(\pi^{2} \epsilon^{3 / 2} / 8\right)\left[1+0.32\left(\nu / \epsilon^{3 / 2}\right)^{-4 / 3}\right]^{-1},
$$

where the factor in square brackets includes a correction to the plateau limit. This was obtained by solving Eq. (6.68) to one higher order in $\epsilon / \nu^{2 / 3}$, and substituting the result into the variational principle. Good agreement with these analytical results was obtained, as shown in Fig. 9. Good agreement was also obtained with the result of a boundary layer calculation, which we now describe.

\section{Boundary layer calculation}

Corrections to the banana regime result were obtained, by a careful treatment of the boundary layer between the trapped and untrapped regions of phase space. As we 


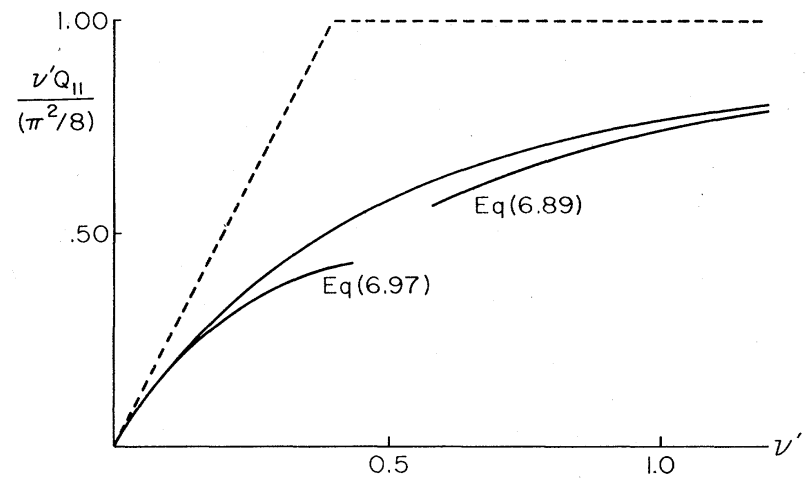

FIG. 9. The function $\nu^{\prime} Q_{11}\left(\nu^{\prime}\right)$, defined by Eq. (6.88), [where $\nu^{\prime} \equiv \nu / \epsilon^{3 / 2}$ and $\nu$ is given by Eq. (6.65)] normalized to the plateau limiting value, $\pi^{2} / 8$. The dashed lines show the banana regime limit, $Q_{11}=3.07$, and the plateau limit $\nu^{\prime} Q_{11}$ $=\pi^{2} / 8$. The correction to the plateau limit given by Eq. (6.89), and the result of the boundary-layer calculation, Eq. (6.97), are also shown.

mentioned in Sec. V.D, the banana regime approximation becomes invalid near the trapping boundary. In order to treat this region properly, the following method was used.

We define the functions $f_{ \pm}$by

$$
g_{1 e}^{+} \pm g_{1 e}^{-}=\left(v /\left|\Omega_{e p}\right|\right) f_{e 0}\left[f_{ \pm} \pm \xi-\nu \theta\right] \text {. }
$$

Then the partial differential equations to be solved may be written approximately as

$$
\partial f_{ \pm} / \partial \varphi= \pm \partial^{2} f_{ \pm} / \partial x^{2},
$$

where the independent variables $x, \varphi$ are defined by

$$
\lambda B_{0}=1-\epsilon+2(\nu / \pi)^{1 / 2}(2 \epsilon)^{1 / 4} x, \quad \varphi=\pi \sin \theta / 2,
$$

and the boundary layer is defined as the region $|x| \sim 1$. The solution of Eq. (6.91) is to be obtained, with the condition $f_{ \pm} \rightarrow 0$ for $x \rightarrow \infty$ (in the trapped region), and with $\partial f_{+} / \partial x$ matched, for $x \rightarrow-\infty$ (in the untrapped region), to the expression obtained from Eq. (5.116). The boundary conditions at $\varphi= \pm \pi$ correspond to particle reflection for $x>0$, and periodicity in $f_{ \pm}$for $x<0$. The solution of this mixed boundary-value problem was obtained by Hinton and Rosenbluth (1973) using the WienerHopf technique. The even part of $g_{1 e}$ is given by the following expression, within the boundary layer

$$
\begin{aligned}
g_{1 e}^{+}= & \frac{v}{\left|\Omega_{e p}\right|} f_{e 0} \frac{\pi}{4} \frac{(\pi \nu)^{1 / 2}}{(2 \epsilon)^{1 / 4}} \int \frac{d k}{2 \pi} e^{-i k x}\left[U(k)-\frac{1}{L(k)}\right] \\
& \times\left\{\exp \left[k^{2}(\varphi+\pi)\right]-\exp \left[-k^{2}(\varphi-\pi)\right]\right\},
\end{aligned}
$$

where $U(k)=\tanh \pi k^{2} / L(k), L(k)$ is analytic and has no zeros in the lower half of the complex $k$ plane, and is such that $L(k) \sim \pi k^{2} \exp (-1.21 i k)$ for $k \rightarrow 0$. This is a highly localized function, which was approximated by a delta-function in the banana regime analysis of Sec. V.D and VI.B.

The boundary-layer contribution to the diffusion coefficient can be obtained by substituting Eq. (6.92) into Eq. (5.118), in place of $g_{1 e}^{(1)}$. The result is proportional to the integral over $v$ of

$$
F \equiv \int d \varphi \int d x \xi \frac{\partial g_{1 e}^{+}}{\partial \varphi}
$$

We evaluate this expression by using an approximate expression for the integrand, $\partial g_{1 e}^{+} / \partial \varphi=\alpha \delta(x-\beta)$, where $\alpha$ and $\beta$ are determined by matching the resulting values of the integrals

$$
\int_{-\infty}^{\infty} d x \partial g_{1 e}^{+} / \partial \varphi, \quad \int_{-\infty}^{\infty} d x x \partial g_{1 e}^{+} / \partial \varphi,
$$

to the exact values, which can be obtained from Eq. (6.92). The result is

$$
\partial g_{1 e}^{+} / \partial \varphi \simeq-\frac{1}{2} \frac{(\pi \nu)^{1 / 2}}{(2 \epsilon)^{1 / 4}} \frac{v}{\left|\Omega_{e p}\right|} f_{e 0} \delta(x-1.21) .
$$

The main effect obtained from the boundary-layer solution is the shift of the maximum of the function $g_{1 e}^{+}$, into the trapped particle region. Since $\xi$ is a decreasing function of $x$, this reduces the value of $F$, Eq. (6.93), by an amount proportional to the shift. (The actual broadening of the function $g_{1 e}^{+}$contributes negligibly.) We thus find the boundary-layer contribution to the diffusion coefficient to be given by Eq. (6.88), with

$$
\left.Q_{11}(\nu)\right|_{\mathrm{bl}}=\left(\pi / 2 \epsilon^{1 / 2}\right) J,
$$

where

$$
\begin{aligned}
J & \equiv \int \frac{d \varphi}{\pi} \int d x \xi \delta(x-1.21), \\
& =1.14 I-0.93 I\left(\nu / \epsilon^{3 / 2}\right)^{1 / 2},
\end{aligned}
$$

with $I \equiv 1.38(2 \epsilon)^{1 / 2}$.

In the banana regime limit $\nu / \epsilon^{3 / 2} \rightarrow 0$, the predominant contribution to $Q_{11}(\nu)$ comes from the boundary layer. [The result $1.14 I$ can also be obtained from the deltafunction term in Eq. (5.123), which is centered at $x=0$.] The untrapped particle region contributes a small negative term, $-0.14 I$, to $I$, while the trapped contribution is negligible, of order $\epsilon^{3 / 2}$. The entire correction to this result, for finite $\nu / \epsilon^{3 / 2}$, comes from the shift of the boundary layer into the trapped region. The odd part of the distribution function $g_{1 e}^{-}$can also be determined from the solution of Eq. (6.91). The result of the careful treatment of the boundary layer is shown in Fig. 7: the discontinuity in the derivative is smoothed out; note the shift in the maximum of the function.

By adding the untrapped particle contribution to Eq. (6.96), we obtain

$$
Q_{11}(\nu)=\left(\pi / 2 \epsilon^{1 / 2}\right) I\left[1-0.93\left(\nu / \epsilon^{3 / 2}\right)^{1 / 2}\right]
$$

for $\nu / \epsilon^{3 / 2} \ll 1$. As shown in Fig. 9, this compares very well with the results of the numerical variational calculation of Hinton and Rosenbluth (1973). As a result of this boundary-layer correction, the diffusion coefficient and the other transport coefficients are reduced by factors $\left[1-a_{m n} \nu_{* e}^{1 / 2}\right]$, where the $a_{m n}$ are constants, and $\nu_{* e}$ is given by Eq. (6.87). These correction factors are included in the more general formulas presented in Sec. VI.F.

\section{Lorentz model}

It is instructive to consider the solution of the Lorentz model kinetic equation for the whole range of collision frequencies. We begin with the assumptions that $Z_{i} \gg 1$, and that electron-electron collisions may be neglected 
FIG. 10. Comparison of $\nu Q_{11}(\nu)$ with $\nu_{*_{e}} K_{11}\left(\nu_{*_{e}}\right)$ for $\epsilon=10^{-2}$. Plotted are (a) $\log \left(\nu^{\prime} Q_{11}\right)$ vs $\log \nu^{\prime}$, where $\nu^{\prime}=\nu / \epsilon^{3 / 2}$ (the dashed lines are the banana, plateau, and collisional regime asymptotic values) and (b) $\log \left(\nu_{* e} K_{11}\right)$ vs $\log \nu_{*_{e}}$, where $\nu_{*_{e}}$ is defined by Eq. (6.87).

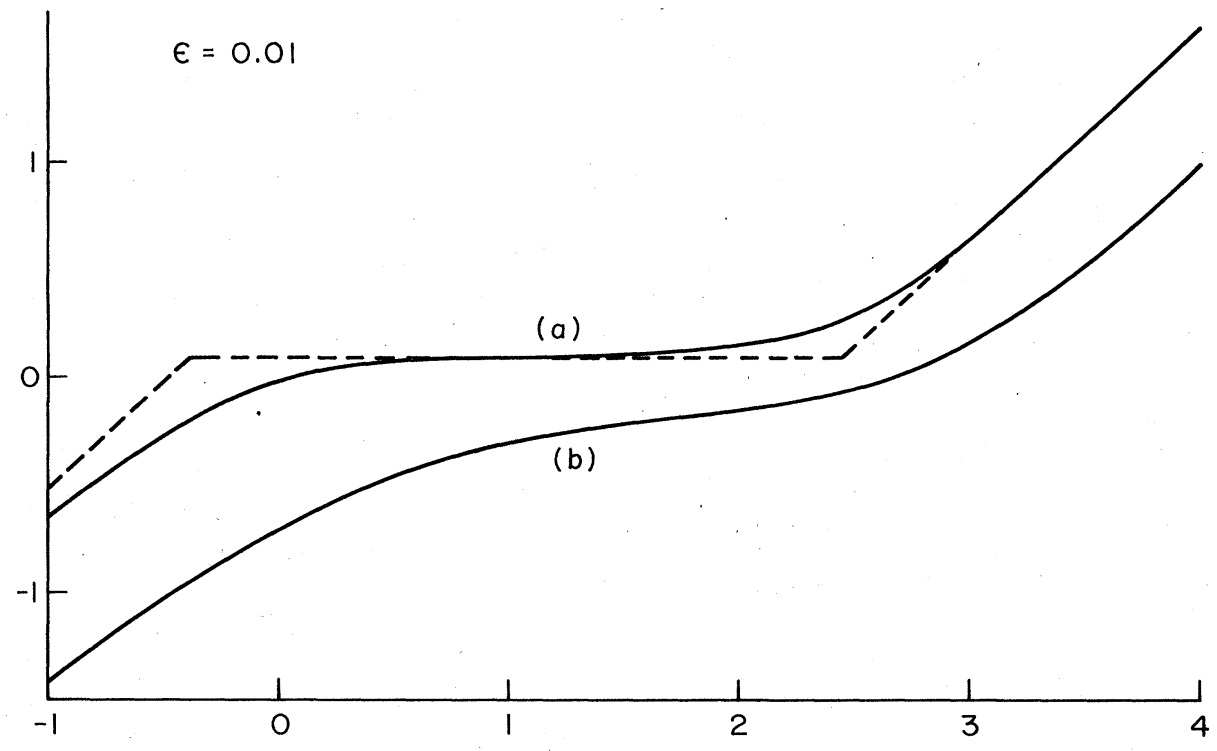

at the outset. Then Eq. (6.63), with $\nu_{e e}=0$, can be regarded as being valid for all values of $\nu$, and can be solved by the numerical method of Hinton and Rosenbluth (1973). The result of calculating $Q_{11}(\nu)$, defined by Eq. (6.88), from the solution so obtained, is shown in Fig. 10, for $\epsilon=0.01$. Also shown, for comparison, is the function $\nu_{* e} K_{11}\left(\nu_{* e}\right)$, defined in Sec. VI.F, which is proportional to the diffusion coefficient $-\left(\alpha_{1}, \alpha_{1 e}\right)$. For this very small value of $\epsilon$, the function $\nu Q_{11}$ has a plateau which is clearly identifiable. The result of integrating over all velocities (and hence over all values of $\nu$ ) is to make the function $\nu_{* e} K_{11}\left(\nu_{* e}\right)$ less flat in the plateau regime than $\nu Q_{11}(\nu)$. Thus, a plateau is not really obtained, because the corrections from the low-energy particles which are in the collisional regime become large before all the higher-energy particles have asymptoted into the plateau regime.

For $\nu / \epsilon^{3 / 2}<0.2$, the computed values of $Q_{11}$ agree well with those given by the analytical expression, Eq. (6.97). For $\nu / \epsilon^{3 / 2}>500$, the computed values agree well with another analytical result, Eq. (6.102) which we shall now derive: the collisional regime limit for the Lorentz model.

By making the substitution

$$
g_{1 e}=\operatorname{Im}\left(h_{1 e} e^{i \theta}\right)
$$

in Eq. (6.63), and neglecting terms of order $\epsilon^{2}$, we have

$$
i \xi h_{1 e}-\frac{1}{2} \nu \frac{d}{d \xi}\left(1-\xi^{2}\right) \frac{d h_{1 e}}{d \xi}=-\frac{\epsilon \nu\left(1+\xi^{2}\right) f_{e 0}}{2\left|\Omega_{e p}\right|},
$$

where $\nu$ is defined in Eq. (6.65) with $\nu_{e e}=0$. In the limit $\nu \gg 1$, we expand $h_{1 e}$ as follows:

$$
h_{1 e}=h_{1 e}^{(-1)}+h_{1 e}^{(0)}+h_{1 e}^{(1)}+\cdots \text {, }
$$

where the superscript gives the power of $1 / \nu$. Then, to lowest order, we have

$$
\frac{d}{d \xi}\left(1-\xi^{2}\right) \frac{d h_{1 e}^{(-1)}}{d \xi}=0,
$$

which implies that $h_{1 e}^{(-1)}$ is isotropic, i.e., a function of $v$ only. The next-order equation is

$$
\frac{1}{2} \nu \frac{d}{d \xi}\left(1-\xi^{2}\right) \frac{d h_{1 e}^{(0)}}{d \xi}=i \xi h_{1 e}^{(-1)}
$$

which may be integrated to give

$$
h_{1 e}^{(0)}=h_{1 e}^{(-1)} \xi / i \nu .
$$

Finally, the equation

$$
\frac{1}{2} \nu \frac{d}{d \xi}\left(1-\xi^{2}\right) \frac{d h_{1 e}^{(1)}}{d \xi}=i \xi h_{1 e}^{(0)}+\frac{\epsilon \nu\left(1+\xi^{2}\right) f_{e 0}}{2\left|\Omega_{e p}\right|}
$$

yields a constraint which determines $h_{1 e}^{(-1)}$ : by integrating over the angular variable $\xi$ from -1 to +1 , and using Eq. (6.100), we obtain

$$
h_{1 e}^{(-1)}=-2 \epsilon\left(v /\left|\Omega_{e p}\right|\right) \nu(v) f_{e 0} .
$$

By substituting this result into Eq. (6.98), calculating the diffusion coefficient $-\left(\alpha_{1}, g_{1 e}\right)$, and comparing with Eq. (6.88), we obtain $Q_{11}=(4 \pi / 3) \epsilon^{3 / 2}$. By carrying the analysis further, we obtain the more accurate result

$$
\nu Q_{11}=(4 \pi / 3) \epsilon^{3 / 2}(\nu+1 / 5 \nu), \text { for } \nu \gg 1 \text {. }
$$

A somewhat paradoxical situation now arises, when we compare (1) the diffusion coefficient obtained by substituting Eq. (6.102) [neglecting the $O(1 / \nu)$ term] into Eq. (6.88), with (2) the result of Sec. V.E, Eq. (5.151) specialized to the Lorentz limit, $Z_{i} \gg 1$. They do not agree: Eq. (6.102) yields $K_{11}=\epsilon^{3 / 2}$ (in the notation defined in Sec. VI.F) while Eq. (5.151) yields $K_{11}=0.46 \epsilon^{3 / 2}$. Thus, the numerical result depends upon which of the two limits, $\nu \rightarrow \infty, Z_{i} \rightarrow \infty$, is taken first.

The physical reason for this nonuniform limit behavior is as follows. When electron-electron collisions are neglected at the outset, the electron distribution function will not approach a Maxwellian (in the absence of forces) in a finite length of time; the choice of $f_{e 0}$ as a Maxwellian is thus not forced on us by the Lorentz model kinetic equation. More to the point, the $O(\nu)$ departure from the equilibrium distribution $f_{e 0}$, due to the requirements of parallel current and heat flow in a torus, is 
not forced to have the form of a Maxwellian with perturbed ( $\theta$-dependent) values of density and temperature. Thus, Eq. (6.101) is not a perturbed Maxwellian, and this is the reason for the numerical discrepancy in the diffusion coefficient: in a sense, the result is a "nonequilibrium" diffusion coefficient.

We conclude that the numerical results shown in Fig. 10 are unphysical in the large $\nu$ limit for two reasons. Firstly, the pitch-angle scattering representation of electron-electron collisions [contained in $Q_{11}(\nu)$ if $\nu$ is given by Eq. (6.65)] is not adequate in the collision-dominated regime. We recognized this by specializing to the case $Z_{i} \gg 1$. Secondly, even when $Z_{i} \gg 1$, the absence of any process, which would tend to Maxwellianize the distribution function, invalidates the results for the transport coefficients. Thus, the numerical results of Hinton and Rosenbluth (1973) are useful only for the bananaplateau regime.

\section{E. The plateau-collisional transition}

We now consider the regime of intermediate to high collisionality, $\nu_{*} \gg 1$, where $\nu_{* e}$ is given by Eq. (6.87) and $\nu_{* i}$ by a similar expression. In the large aspectratio, circular cross section case which we are considering, the $\theta$ dependence of the distribution functions is simply sinusoidal, so that only the dependence on the velocity variables $v, \xi$ remains to be determined.

\section{Electron transport}

We begin with Eq. (6.63), with the exact collision term $C_{e e}$ in place of the pitch-angle scattering term. By expanding in the inverse aspect ratio

$$
g_{n e}=g_{n e}^{(1)}+g_{n e}^{(2)}+\cdots,
$$

and writing the $\theta$ dependence of the first-order term explicitly

$$
g_{n e}^{(1)}=\operatorname{Im}\left(h_{n e} e^{i \theta}\right),
$$

we have (for $n=1$, for example)

$$
i \xi h_{1 e}-\left(r B / B_{\mathrm{po}} v\right) C_{e} h_{1 e}=-\frac{\epsilon v\left(1+\xi^{2}\right)}{2\left|\Omega_{e p}\right|} f_{e 0},
$$

where $C_{e}$ is given by Eq. (1.19). The expressions for the inner products now involve integration only over velocity; for example, the diffusion coefficient is

$$
-\left(\alpha_{1}, g_{1 e}\right)=\frac{-1}{2\left|\Omega_{e 0}\right| R_{0}} \int d^{3} v \frac{v^{2}}{2}\left(1+\xi^{2}\right) \operatorname{Re} h_{1 e},
$$

where $\left|\Omega_{e 0}\right| \equiv e B_{0} / m_{e} c$. We note that the exact solution of Eq. (6.103) would still be difficult, because of the complexity of the Fokker-Planck collision operator for electron-electron collisions. An approximate method of solution will therefore be discussed subsequently.

\section{Ion transport}

We now consider Eq. (6.78) for the ion distribution function $g_{2 i}$. As in the plateau limit, the substitution of Eq. (6.81) leads to Eq. (6.82), but now the exact ion-ion collision operator $C_{i i}$ must be retained. The value of $y$, which appears in Eq. (6.81), is again determined by Eq. (6.80), which is a consequence of the fact that ion-ion collisions conserve momentum. By expanding in powers of $\epsilon$

$$
H_{i}=H_{i}^{(1)}+H_{i}^{(2)}+\cdots
$$

and writing the $\theta$ dependence of the first-order term explicitly

$$
H_{i}^{(1)}=\operatorname{Im}\left(h_{2 i} e^{i \theta}\right),
$$

we obtain

$$
i \xi h_{2 i}-\left(r B / B_{\mathrm{po}} v\right) C_{i i} h_{2 i}=\left(v^{2} / v_{\mathrm{th} i}^{2}-y\right) \frac{\epsilon v\left(1+\xi^{2}\right)}{2 \Omega_{i p}} f_{i_{0}} .
$$

An approximate method of solution will be described subsequently.

The ion thermal conductivity is given by

$-\left(\beta_{2}, g_{2 i}\right)=\frac{1}{2 \Omega_{i_{0}} R_{0}} \int d^{3} v \frac{v^{2}}{2}\left(v^{2} / v_{\mathrm{th} i}^{2}-5 / 2\right)\left(1+\xi^{2}\right) \operatorname{Re} h_{2 i}$.

The constraint, Eq. (6.80), which determines $y$, may be written as follows, after integrating by parts

$$
\int d^{3} v v^{2}\left(1-3 \xi^{2}\right) \operatorname{Re}_{2 i}=0 .
$$

We note that the mirror force term (proportional to $\epsilon \sin \theta$ ) in Eq. (6.82) has been neglected, in obtaining Eq. (6.105); it has been used in obtaining the constraint, Eq. (6.107). In the intermediate-to-high collisionality regime, the majority of particles (the well-untrapped ones) which contribute to diffusion are only slightly affected by the mirror force, so it can be treated as a perturbation. In the absence of any parallel magnetic field gradient, however, the ion parallel flow velocity would be arbitrary, corresponding to the choice of reference frame. The small mirror force removes this degeneracy, in a toroidal confinement system, and enables the parallel velocity [and hence $y$, in Eq. (6.81)] to be uniquely determined.

\section{Model like-particle collision operator}

The above kinetic equations [Eq. (6.103) and its $n=2$ and $n=3$ counterparts, and Eq. (6.105)] were solved by Rawls, Chu, and Hinton (1975) by using a model likeparticle collision operator. Since this operator may be generally useful, we give some of the details

$$
\begin{array}{r}
C_{i i} f_{i_{1}} \equiv \bar{\nu}_{i i}(v)[ \\
{\left[\mathcal{L} f_{i_{1}}-f_{i_{1}}+\sqrt{2}\left(v_{\|} / v_{\text {thi }}\right) p f_{i_{0}}\right.} \\
\left.+\rho f_{i_{0}}+s\left(v^{2} / v_{\text {th }}^{2}-5 / 2\right) f_{i_{0}}\right],
\end{array}
$$

where $\mathscr{L}$ is the pitch-angle scattering operator given by Eq. (5.5). The collision frequency is a function of velocity $v$, defined here as

$$
\bar{\nu}_{i i}(v)=\frac{\beta}{\tau_{i} \lambda^{3}}\left(\frac{v_{\text {th } i}}{v}\right)^{3} \mu\left(\frac{\lambda v}{v_{\text {thi }}}\right),
$$

where $\tau_{i}$ is given by Eq. (6.42), and

$$
\mu(x) \equiv \operatorname{erf}(x)-\left(2 / \pi^{1 / 2}\right) x e^{-x^{2}} .
$$

This particular function was chosen so that $\bar{\nu}_{i i}(v)$, with $\lambda=1$, gives the rate of slowing down of a test ion by col- 
lisions with other ions (Trubnikov, 1965). The values of $\beta$ and $\lambda$ were determined by using the corresponding model electron-electron collision operator to solve the classical parallel transport problem of Spitzer and Harm (1953). By finding a best fit to the values of $\kappa_{11}$, $\kappa_{12}^{\prime}$, and $\kappa_{22}$ given in Table $\Pi$, for $Z_{i}=1$, the values $\beta=0.96, \lambda=0.55$ were obtained. The parameters $p, \rho$, and $s$ are to be determined by the requirements of conservation of particle number, momentum, and energy in like-particle collisions

$$
\int d^{3} v\left(1, m_{i} v_{\|}, m_{i} v^{2} / 2\right) C_{i i} f_{i_{1}}=0
$$

It can be shown that this model collision operator is self-adjoint and satisfies the $H$ theorem.

A convenient method of solution of Eqs. (6.103) and (6.105), with the model operator $C_{e e}$ or $C_{i i}$, involves expansion of the unknown functions, $h_{1 e}$ and $h_{2 i}$, in a series of Legendre polynomials $P_{l}(\xi)$. The expansion coefficients satisfy recursion relations which can be solved by a computer, assuming the series to be truncated at a large value of $l$. The self-consistent solution for the parameters $p, \rho$, and $s$ involves only the coefficients of $P_{0}(\xi)$ and $P_{1}(\xi)$, while the transport coefficients are obtained from the coefficients of $P_{0}(\xi)$ and $P_{2}(\xi)$.

The numerical results obtained by this method agree fairly well in the plateau limit with those given in Sec. VI.D. In the collisional regime, the limiting values agree well with the results of Sec. V.E., specialized to the case of large aspect ratio and circular cross section. We note that the quantity in square brackets in Eqs. (5.151)-(5.153) and Eq. (5.161) is easily evaluated in this case to give

$$
\left\langle h^{2}\right\rangle-\left\langle h^{-2}\right\rangle^{-1}=2 \epsilon^{2} \text {. }
$$

Least-squares fits were made, to simple analytical formulas for the collision frequency dependence, and these are incorporated in the more general results to be presented in Sec. VI.F.

\section{F. Summary of results}

We have obtained transport coefficients for large aspect-ratio tokamaks in several regimes of collisionality. All regimes may be relevant in a given plasma, at different minor radii, since the collisionality is defined in terms of minor radius, density and temperature. To be most useful, the results should be expressed as continuous functions of collisionality. In this section, we give simple analytical expressions, obtained by least-squares fits to the previous results, which express the transport coefficients as continuous functions of collisionality. The electron and ion collisionality parameters used here are defined by

$$
\nu_{* e} \equiv \sqrt{2} r B_{0} /\left(B_{\mathrm{po}} v_{\text {the }} \tau_{e} \epsilon^{3 / 2}\right),
$$

where $v_{\text {the }} \equiv\left(2 T_{e} / m_{e}\right)^{1 / 2}$, and $\tau_{e}$ is given by Eq. (5.4), and

$$
\nu_{* i} \equiv \sqrt{2} r B_{0} /\left(B_{\mathrm{po}} v_{\mathrm{th} i} \tau_{i} \epsilon^{3 / 2}\right),
$$

where $v_{\mathrm{th} i} \equiv\left(2 T_{i} / m_{i}\right)^{1 / 2}$, and $\tau_{i}$ is given by Eq. (6.42).

\section{Electron fluxes}

Rather than fit the inner products $\left(\alpha_{m}, g_{n e}\right)$, it was found more convenient to fit a dimensionless set of electron transport coefficients $K_{m n}$, defined as follows

$$
\begin{aligned}
& -\left(\alpha_{1}, g_{1 e}\right)=n_{e} \epsilon^{1 / 2}\left(\rho_{e \theta}^{2} / \tau_{e}\right) K_{11}, \\
& -\left(\alpha_{1}, g_{3 e}\right)-\frac{5}{2}\left(\alpha_{1}, g_{1 e}\right)=n_{e} \epsilon^{1 / 2}\left(\rho_{e \theta}^{2} / \tau_{e}\right) K_{12}, \\
& -\left(\alpha_{2}, g_{2 e}\right)-5\left(\alpha_{1}, g_{2 e}\right)-\frac{25}{4}\left(\alpha_{1}, g_{1 e}\right)=n_{e} \epsilon^{1 / 2}\left(\rho_{e \theta}^{2} / \tau_{e}\right) K_{22},
\end{aligned}
$$

$$
\begin{aligned}
& -\left(\alpha_{1}, g_{3 e}\right)=\epsilon^{1 / 2}\left(n_{e} c / B_{\mathrm{po}}\right) K_{13}, \\
& -\left(\alpha_{2}, g_{3 e}\right)-\frac{5}{2}\left(\alpha_{1}, g_{3 e}\right)=\epsilon^{1 / 2}\left(n_{e} c / B_{\mathrm{po}}\right) K_{23}, \\
& -\left(\alpha_{3}, g_{3 e}\right)=\epsilon^{1 / 2}\left(\sigma_{\|} / T_{e}\right) K_{33},
\end{aligned}
$$

where $\epsilon \equiv r / R_{0}, \quad \rho_{e \theta}^{2}=2 m_{e} T_{e} c^{2} / e^{2} B_{\mathrm{po}}^{2}, \tau_{e}$ is given by Eq. (5.4), and $\sigma_{\|}$is given by Eq. (5.66).

In terms of these coefficients, the electron transport relations, Eqs. (5.70)-(5.72) may be written as follows, using the weak-coupling approximation and Eq. (5.83)

$$
\begin{aligned}
& \Gamma_{e}=-n_{e} \epsilon^{1 / 2}\left(\rho_{e \theta}^{2} / \tau_{e}\right)\left\{K_{11} A_{1 e}^{\prime}+K_{12} \frac{\partial}{\partial r} \ln T_{e}\right\} \\
&-K_{13} n_{e} \epsilon^{1 / 2} c\left\langle E_{\|} / h\right\rangle / B_{\mathrm{po}}, \\
& q_{e}+\frac{5}{2} T_{e} \Gamma_{e}=-n_{e} T_{e} \epsilon^{1 / 2}\left(\rho_{e \theta}^{2} / \tau_{e}\right)\left\{K_{12} A_{1 e}^{\prime}+K_{22} \frac{\partial}{\partial r} \ln T_{e}\right\} \\
&-K_{23} n_{e} T_{e} \epsilon^{1 / 2} c\left\langle E_{\|} / h\right\rangle / B_{\mathrm{po}}, \\
&\left\langle\left(J_{\|}-J_{\| s}\right) / h\right\rangle=-n_{e} T_{e} \epsilon^{1 / 2}\left(c / B_{\mathrm{po}}\right)\left\{K_{13} A_{1 e}^{\prime}+K_{23} \frac{\partial}{\partial r} \ln T_{e}\right\} \\
&-K_{33} \epsilon^{1 / 2} \sigma_{\|}\left\langle E_{\|} / h\right\rangle .
\end{aligned}
$$

Here we have used $A_{3 e} \simeq\left\langle E_{\|} / h\right\rangle$, which follows from the approximation $\left\langle B^{2}\right\rangle \simeq B_{0}^{2}$. We have combined the forces $A_{1 e}$ and $A_{4 e}$ in a single force

$$
\begin{aligned}
A_{1 e}^{\prime}= & \frac{\partial}{\partial r} \ln p_{e}-\frac{5}{2} \frac{\partial \ln T_{e}}{\partial r} \\
& +\left(T_{i} / Z_{i} T_{e}\right)\left[\frac{\partial}{\partial r} \ln p_{i}-\frac{\left(\beta_{1}, g_{2 i}\right)}{1+\nu_{* e}^{2} \epsilon^{2}} \frac{\partial \ln T_{i}}{\partial r}\right]
\end{aligned}
$$

[where the extra term $-\frac{5}{2}\left(\partial \ln T_{e} / \partial r\right)$ corresponds to the use of $q_{e}+\frac{5}{2} T_{e} \Gamma_{e}$ as a flux, rather than $q_{e}$, and maintains the Onsager symmetry.] The use of the combined force $A_{1 e}^{\prime}$ affords a considerable simplification of the above formulas, and is motivated as follows. For $n=1$ or 2 , we have $\left(\alpha_{n}, g_{4 e}\right) \simeq\left(\alpha_{n}, g_{1 e}\right)$ in the banana-plateau regime $\left(\nu_{* e} \ll \epsilon^{-3 / 2}\right)$, while $\left(\alpha_{n}, g_{4 e}\right) /\left(\alpha_{n}, g_{1 e}\right) \sim \nu_{* e}^{-2} \epsilon^{-3} \ll 1$ in the collisional regime $\left(\nu_{* e} \gg \epsilon^{-3 / 2}\right)$. Since the behavior of the coefficients $\left(\alpha_{1}, g_{4 e}\right)$ and $\left(\alpha_{2}, g_{4 e}\right)$ in the plateau-collisional transition regime is not known, we use the simple approximation

$$
\left(\alpha_{n}, g_{4 e}\right) \simeq\left(\alpha_{n}, g_{1 e}\right) /\left(1+\nu_{* e}^{2} \epsilon^{3}\right) .
$$

For the case $n=3$, however, we have $\left(\alpha_{3}, g_{4 e}\right) /\left(\alpha_{3}, g_{1 e}\right) \sim 1$ in all collisionality regimes, but since the coefficient of $A_{1 e}^{\prime}$ in $\left\langle\left(J_{\|}-J_{\| s}\right) / h\right\rangle$ is small in the collisional regime $\left[\left(\alpha_{3}, g_{1 e}\right) \sim\left(\alpha_{3}, g_{4 e}\right) \sim \nu_{* e}^{-2} \epsilon^{-3}\right]$, the error introduced by using Eq. (6.124) is small. An expression for $\left(\beta_{1}, g_{2 i}\right)$, 
which also appears in Eq. (6.123), will be given subsequently.

The approximate analytic expressions, to which the electron transport coefficient results are fitted, are as follows. For $m$ or $n=1$ or 2 ,

$K_{m n}=K_{m n}^{(0)}\left\{\frac{1}{1+a_{m n} \nu_{* e}^{1 / 2}+b_{m n} \nu_{* e}}+\frac{\epsilon^{3 / 2}\left(c_{m n}^{2} / b_{m n}\right) \nu_{* e} \epsilon^{3 / 2}}{1+c_{m n} \nu_{* e} \epsilon^{3 / 2}}\right\}$

For $n=3$,

$$
K_{m_{3}}=K_{m_{3}}^{(0)}\left[1+a_{m_{3}} \nu_{* e}^{1 / 2}+b_{m_{3}} \nu_{* e}\right]^{-1}\left[1+c_{m_{3}} \nu_{* e} \epsilon^{3 / 2}\right]^{-1}
$$

The numerical coefficients, $K_{m n}^{(0)}, a_{m n}, b_{m n}$, and $c_{m n}$, were obtained by considering the following two limiting cases.

(i) $\nu_{* e} \ll \epsilon^{-3 / 2}$ (banana-plateau regime)

$$
K_{m n} \simeq K_{m n}^{(0)}\left[1+a_{m n} \nu_{* e}^{1 / 2}+b_{m n} \nu_{* e}\right]^{-1} \text {. }
$$

The results of Hinton and Rosenbluth (1973) for $\epsilon \ll 1$, were fitted to such functions. The term involving $\nu_{* e}^{1 / 2}$ reflects the role of the boundary layer, as mentioned previously. The values of $K_{m n}^{(0)}$ and $a_{m n}$ so obtained are listed in Table III. Note that the values of $K_{m n}^{(0)}$ were obtained from least-squares fits, in the range $10^{-2}<\nu_{* e}$ $<10$; they do not agree exactly with the banana regime values, from Eqs. (6.28)-(6.33), which apply in the limit $\nu_{* e} \rightarrow 0$. Also listed in Table MI are the values of $b_{33}$ and $c_{33}$ obtained by Hinton and Rosenbluth (1973), since these are the only known values of these coefficients.

(ii) $\nu_{* e} \gg 1$ (plateau-collisional regime): For $m$ or $n=1$ or 2 ,

TABLE III. Numerical coefficients in Eqs. (6.125) and (6.126): the results of least-squares fits to the transport coefficients.

\begin{tabular}{ccccc}
\hline \hline $\begin{array}{c}\text { A. } \begin{array}{l}Z_{i}=1 \\
m n\end{array} \\
\boldsymbol{K}_{m n}^{(0)}\end{array}$ & $a_{m n}$ & $b_{m n}$ & $c_{m n}$ \\
\hline 11 & 1.04 & 2.01 & 1.53 & 0.89 \\
12 & 1.20 & 0.76 & 0.67 & 0.56 \\
22 & 2.55 & 0.45 & 0.43 & 0.43 \\
13 & 2.30 & 1.02 & 1.07 & 1.07 \\
23 & 4.19 & 0.57 & 0.61 & 0.61 \\
33 & 1.83 & 0.68 & 0.32 & 0.66 \\
\hline
\end{tabular}

B. $Z_{i}=2$

\begin{tabular}{lllll}
$m n$ & $K_{m n}^{(0)}$ & $a_{m n}$ & $b_{m n}$ & $c_{m n}$ \\
\hline 11 & 0.86 & 2.18 & 1.17 & 0.79 \\
12 & 0.95 & 0.78 & 0.50 & 0.51 \\
22 & 1.99 & 0.46 & 0.26 & 0.34 \\
13 & 1.87 & 0.89 & 0.62 & 0.69 \\
23 & 3.72 & 0.52 & 0.34 & 0.38 \\
33 & 1.56 & 0.56 & 0.25 & 0.58 \\
\hline
\end{tabular}

C. $Z_{i}=4$

\begin{tabular}{lllll}
$m n$ & $K_{m n}^{(0)}$ & $a_{m n}$ & $b_{m n}$ & $c_{m n}$ \\
\hline 11 & 0.76 & 2.30 & 0.98 & 0.74 \\
12 & 0.83 & 0.80 & 0.42 & 0.48 \\
22 & 1.71 & 0.46 & 0.22 & 0.30 \\
13 & 1.65 & 0.79 & 0.56 & 0.51 \\
23 & 3.54 & 0.48 & 0.33 & 0.28 \\
33 & 1.42 & 0.47 & 0.20 & 0.51 \\
\hline \hline
\end{tabular}

$K_{m n} \simeq \epsilon^{3 / 2}\left(c_{m n} / b_{m n}\right) K_{m n}^{(0)}\left\{1+\frac{1}{c_{m n} \nu_{* e} \epsilon^{3 / 2}\left(1+c_{m n} \nu_{* e} \epsilon^{3 / 2}\right)}\right\}$

For $n=3$,

$$
K_{m 3} \simeq\left(K_{m 3}^{(0)} / b_{m n} \nu_{* e}\right)\left[1+c_{m n} \nu_{* e} \epsilon^{3 / 2}\right]^{-1} .
$$

The results of Rawls, Chu, and Hinton (1975) for $\epsilon \ll 1$ were fitted to such expressions; their values for $b_{m n}$ and $c_{m n}$ are listed in Table III (except for $b_{33}$ and $c_{33}$, as mentioned previously). Again, note that these values were obtained from least-squares fits, so they do not exactly agree with the collisional regime values given by Eqs. (5.151)-(5.153).

Since these two limiting cases represent regimes of collisionality which overlap, when $\epsilon \ll 1$, we may regard the expressions given in Eqs. (6.125) and (6.126) to be valid for all values of the collisionality parameter $\nu_{* e}$. It then seems reasonable to extrapolate the results to finite values of $\epsilon$. In Fig. 11, we show the result of using the interpolation formula, Eq. (6.125), for a value $\epsilon=0.2$, which makes it possible to see all three collisionality regimes on the same linear scale. The diffusion coefficient $-\left(\alpha_{1}, g_{1 e}\right)$, which is proportional to $\nu_{* e} K_{11}\left(\nu_{* e}\right)$, is plotted; the ordinate gives the diffusion coefficient normalized to its plateau value (called $\hat{D}$ in the figure). Also shown for comparison are the banana, plateau, and collisional regime asymptotes, the banana-plateau transition formula, Eq. (6.127), and the plateau-collisional transition formula, Eq. (6.128).

\section{Ion fluxes}

A dimensionless ion thermal conductivity coefficient $K_{2}$ may be defined as

$$
-\left(\beta_{2}, g_{2 i}\right)=n_{i} \epsilon^{1 / 2}\left(\rho_{i \theta}^{2} / \tau_{i}\right) K_{2},
$$

where $\rho_{i \theta}^{2} \equiv 2 m_{i} T_{i} c^{2} / Z_{i}^{2} e^{2} B_{\text {po }}^{2}$, and $\tau_{i}$ is given by Eq. (6.42). In terms of this coefficient, the ion heat flux, Eq. (5.84), may be written as follows (in the weak-coupling approximation)

$$
q_{i}=-K_{2} n_{i} \epsilon^{1 / 2}\left(\rho_{i \theta}^{2} / \tau_{i}\right) \frac{\partial T_{i}}{\partial r}-\frac{\left(\beta_{1}, g_{2 i}\right) T_{i} \Gamma_{e} / Z_{i}}{1+\nu_{* e}^{2} \epsilon^{3}}
$$

Here we have used the Onsager relation $\left(\beta_{2}, g_{1 i}\right)$ $=-\left(\beta_{1}, g_{2 i}\right)$. We have used the approximation of Eq. (5.75) for $A_{1 i}$, which gives, on comparison with Eq. (5.70),

$$
A_{1 i} \simeq \frac{\Gamma_{e} / Z_{i}}{1+\nu_{* e}^{2} \epsilon^{3}}
$$

The following approximate analytic expression for the coefficient $K_{2}$ was fitted to the results of Hinton and Rosenbluth (1973), for the banana-plateau regime $\left(\nu_{* i} \ll \epsilon^{-3 / 2}\right)$ and to the results of Rawls, Chu and Hinton, for the plateau-collisional regime $\left(\nu_{* i} \gg 1\right)$ :

$$
K_{2}=K_{2}^{(0)}\left\{\frac{1}{1+a_{2} \nu_{* i}^{1 / 2}+b_{2} \nu_{* i}}+\frac{\epsilon^{3 / 2}\left(c_{2}^{2} / b_{2}\right) \nu_{* i} \epsilon^{3 / 2}}{1+c_{2} \nu_{* i} \epsilon^{3 / 2}}\right\} .
$$

The numerical values of $K_{2}^{(0)}, a_{2}, b_{2}$, and $c_{2}$ are as fol- 
lows

$$
K_{2}^{(0)}=0.66, a_{2}=1.03, \quad b_{2}=0.31, \quad c_{2}=0.74 .
$$

The mean ion parallel velocity, averaged over a magnetic surface, is obtained from Eqs. (5.46) and Eq.

(5.83), in the weak-coupling approximation:

$$
\left\langle u_{i \|}\right\rangle=\frac{c T_{i}}{Z_{i} e B}\left\{\left(\beta_{1}, g_{2 i}\right) \frac{\partial \ln T_{i}}{\partial r}-\frac{\partial \ln p_{i}}{\partial r}-\frac{Z_{i} e}{T_{i}} \frac{\partial\langle\Phi\rangle}{\partial r}\right\} .
$$

The coefficient $\left(\beta_{1}, g_{2 i}\right)$, is equal to 1.17 in the banana regime, -0.5 in the plateau limit, and -2.1 in the collisional regime (in terms of ion collisionality). The $\nu_{* i}$ dependence in the banana-plateau regime was obtained by Hinton and Rosenbluth (1973); an approximate fit is (for $\nu_{* i} \ll \epsilon^{-3 / 2}$ )

$$
\left(\beta_{1}, g_{2 i}\right) \simeq \frac{1.17-0.35 \nu_{* i}^{1 / 2}}{1+0.7 \nu_{* i}^{1 / 2}} \equiv \frac{5}{2}-y .
$$

Since the $\nu_{* i}$ dependence in the plateau-collisional regime is not known, we use the simple formula

$$
\left(\beta_{1}, g_{2 i}\right) \simeq \frac{\frac{5}{2}-y-2.1 \nu_{* i}^{2} \epsilon^{3}}{1+\nu_{* i}^{2} \epsilon^{3}},
$$

where $\frac{5}{2}-y$ is given by Eq. (6.135).

\section{Weak-coupling approximation}

As a check on the weak-coupling approximation of Sec. V.B.4, we now state the conditions under which $F-1$, as given by Eq. (5.86), is small. Since the electrons and ions may be in different collisionality regimes, in general, we consider four possibilities. In Table IV, we give estimates for $F-1$ when either the electrons or the ions may be in either the banana or the collisional regime. The expressions used for the inner products $\left(\alpha_{4}, g_{4 e}\right)$ and $\left(\beta_{1}, g_{1 i}\right)$ are obtained as follows. If the electrons are in the banana regime, we use Eq. (6.28); if they are in the collisional regime, we use Eq. (5.155). If the ions are in the banana regime, we note that the equa-

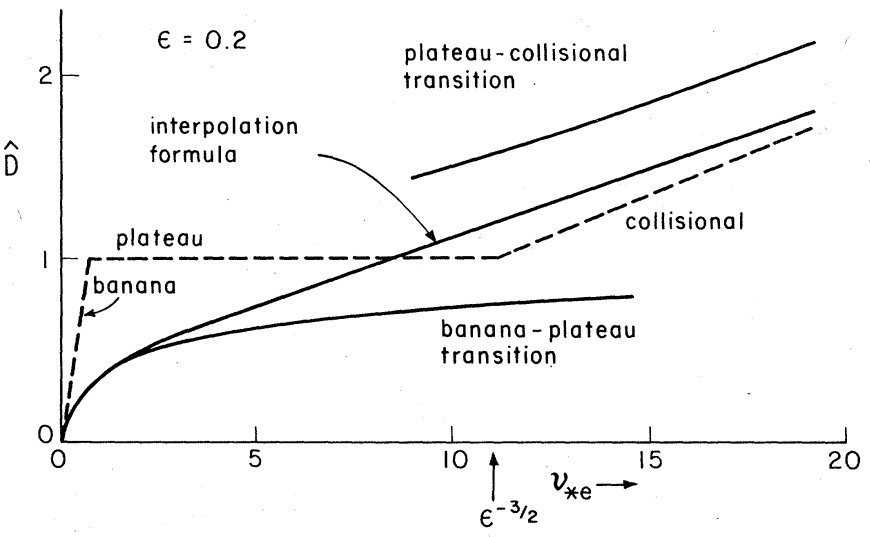

FIG. 11. Comparison of asymptotic forms of the diffusion coefficient $\hat{D}$ (normalized to the plateau value), in the various collisionality regimes, with the interpolation formula, Eq. (6.125). These were derived with the assumption $\epsilon \ll 1$, and have been extrapolated to a finite inverse aspect ratio, $\epsilon=0.2$.

tion satisfied by the function $g_{1 i}$, as given in Sec. V.D.5, has not been solved; therefore, we use the estimate

$$
\left(\beta_{1}, g_{1 i}\right) \sim\left(\epsilon^{1 / 2} n_{i} \rho_{i \theta}^{2} / \tau_{i}\right)^{-1}
$$

for the banana regime. If the ions are in the collisional regime, we use Eq. (5.164). As can be seen from Table IV, the weak-coupling approximation is most difficult to satisfy when both species are in the collisional regime. As mentioned in Sec. V.E, however, this condition requires only $T_{e}<5 T_{i}$ for the hydrogen mass ratio, with $Z_{i}=1$.

\section{Two ion species problem}

As we mentioned in Sec. V.B.5, the weak-coupling approximation is not generally valid for the problem of two ion species. The complete formulae given in that section must be used to obtain the particle and heat fluxes, in general. These are

$$
\begin{aligned}
\Gamma_{a}= & {\left.\left[\alpha_{1}, g_{1}\right)-\left(\alpha_{1}, g_{3 a}\right)^{2}\left(\beta_{1}, g_{1 b}\right)\left(Z_{a}^{2} / Z_{b}^{2} F\right)\right]\left[\frac{\partial \ln p_{a}}{\partial r}-\frac{Z_{a}}{Z_{b}} \frac{\partial \ln p_{b}}{\partial r}\right] } \\
+ & {\left[\left(\alpha_{1}, g_{2 a}\right)-\left(\alpha_{1}, g_{3 a}\right)\left(\alpha_{2}, g_{3}\right)\left(\beta_{1}, g_{1 b}\right)\left(Z_{a}^{2} / Z_{b}^{2} F\right)+\left(\alpha_{1}, g_{3 a}\right)\left(\beta_{1}, g_{2 b}\right)\left(Z_{a} / Z_{b} F\right)\right] \frac{\partial \ln T}{\partial r}, } \\
q_{a} / T= & {\left[\left(\alpha_{1}, g_{2 a}\right)-\left(\alpha_{1}, g_{3 a}\right)\left(\alpha_{2}, g_{3 a}\right)\left(\beta_{1}, g_{1 b}\right)\left(Z_{a}^{2} / Z_{b}^{2} F\right)\right]\left[\frac{\partial \ln p_{a}}{\partial r}-\frac{Z_{a}}{Z_{b}} \frac{\partial \ln p_{b}}{\partial r}\right] } \\
+ & {\left[\left(\alpha_{2}, g_{2 a}\right)-\left(\alpha_{2}, g_{3 a}\right)^{2}\left(\beta_{1}, g_{1 b}\right)\left(Z_{a}^{2} / Z_{b}^{2} F\right)+\left(\alpha_{2}, g_{3 a}\right)\left(\beta_{1}, g_{2 b}\right)\left(Z_{a} / Z_{b} F\right)\right] \frac{\partial \ln T}{\partial r}, } \\
q_{b} / T= & \left(\alpha_{1}, g_{3 a}\right)\left(\beta_{1}, g_{2 b}\right)\left(Z_{a} / Z_{b} F\right)\left[\frac{\partial \ln p_{a}}{\partial r}-\frac{Z_{a}}{Z_{b}} \frac{\partial \ln p_{b}}{\partial r}\right] \\
& +\left[\left(\beta_{2}, g_{2 b}\right)+\left(\alpha_{2}, g_{3 a}\right)\left(\beta_{1}, g_{2 b}\right)\left(Z_{a} / Z_{b} F\right)+\left(\alpha_{3}, g_{3 a}\right)\left(\beta_{1}, g_{2 b}\right)^{2}\left(Z_{a}^{2} / Z_{b}^{2} F\right)\right] \frac{\partial \ln T}{\partial r},
\end{aligned}
$$

where $F \equiv 1+\left(Z_{a}^{2} / Z_{b}^{2}\right)\left(\alpha_{3}, g_{3 a}\right)\left(\beta_{1}, g_{1 b}\right)$, and we have used $T_{b} \simeq T_{a} \equiv T$, and the Onsager relations, Eqs. (5.98) and (5.99).

The inner products may be obtained in special cases by transposing the results given earlier in this section, i.e., by replacing $m_{e}$ by $m_{a}, m_{i}$ by $m_{b}$, etc. (We note, however, that the required inner products are not yet available in the plateau-collisional transition regime.)

Rev. Mod. Phys., Vol. 48, No. 2, Part I, April 1976 
TABLE IV. Estimates of $F-1$, whose smallness is required for the weak-coupling approximation to be valid.

\begin{tabular}{lcc}
\hline & $\begin{array}{c}\text { Electrons in } \\
\text { banana regime } \\
\left(\nu_{* e} \ll 1\right)\end{array}$ & $\begin{array}{c}\text { Electrons in } \\
\text { collisional regime } \\
\left(\nu_{* e} \gg \epsilon^{-3 / 2}\right)\end{array}$ \\
\hline $\begin{array}{c}\text { Ions in banana regime } \\
\left(\nu_{* i} \ll 1\right)\end{array}$ & $\frac{1}{Z_{i}}\left(\frac{m_{e}}{m_{i}}\right)^{1 / 2}\left(\frac{T_{i}}{T_{e}}\right)^{3 / 2}$ & $\frac{Z_{i}}{\epsilon^{3 / 2}}\left(\frac{m_{e}}{m_{i}}\right)^{1 / 2}\left(\frac{T_{e}}{T_{i}}\right)^{1 / 2} / \nu_{* e} \nu_{* i}$ \\
$\begin{array}{c}\text { Ions in collisional regime } \\
\left(\nu_{* i} \gg \epsilon^{-3 / 2}\right)\end{array}$ & $\epsilon^{3 / 2} Z_{i}\left(\frac{m_{e}}{m_{i}}\right)^{1 / 2}\left(\frac{T_{e}}{T_{i}}\right)^{1 / 2} \nu_{* e} \nu_{* i}$ & $Z_{i}^{3}\left(\frac{m_{e}}{m_{i}}\right)^{1 / 2}\left(\frac{T_{e}}{T_{i}}\right)^{5 / 2}$ \\
\hline
\end{tabular}

This replacement procedure gives the same results as, for example, those obtained by Connor (1973) for the banana regime, and those obtained by Rutherford (1974) for the collisional regime. The poloidal gyroradii $\rho_{e \theta}$ and $\rho_{i \theta}$ must be replaced by

$$
\rho_{a \Theta} \equiv\left(2 m_{a} T\right)^{1 / 2} c / Z_{a} e B_{\mathrm{po}}
$$

and

$$
\rho_{b \Theta} \equiv\left(2 m_{b} T\right)^{1 / 2} c / Z_{b} e B_{\mathrm{po}},
$$

respectively. The densities $n_{e}, n_{i}$ are to be replaced by $n_{a}, n_{b}$. The electron collision time $\tau_{e}$, given by Eq. (5.4), is to be replaced by $\tau_{a b}$, where

$$
\tau_{a b}^{-1}=\frac{4}{3} \frac{(2 \pi)^{1 / 2} n_{b} Z_{a}^{2} Z_{b}^{2} e^{4} \ln \Lambda}{m_{a}^{1 / 2} T^{3 / 2}}
$$

while the ion collision time of Eq. (6.42), must be replaced by $\tau_{b b}$, where

$$
\tau_{b b}^{-1}=\frac{4}{3} \pi^{1 / 2} \frac{n_{b} Z_{b}^{4} e^{4} \ln \Lambda}{m_{b}^{1 / 2} T^{3 / 2}} .
$$

The charge-neutrality condition $n_{e}=Z_{i} n_{i}$ has no counterpart in the two ion species problem; the ion charge number $Z_{i}$ in certain expressions must be replaced by the parameter (Connor, 1973)

$$
\alpha=n_{b} Z_{b}^{2} / n_{a} Z_{a}^{2} \text {. }
$$

The banana regime coefficients, for example, Eqs. (6.28)-(6.30), are expressed as continuous functions of $\alpha$ by replacing $1+0.53 / Z_{i}$ by $1+0.53 / \alpha$, etc.

Equations (6.138)-(6.140) may be simplified considerably when both ion species are in the collisional regime. Using Eqs. (5.155), (5.156), and (5.164), we find

$$
\begin{aligned}
& \Gamma_{a}=\left(\alpha_{1}, g_{1 a}\right)\left[\frac{\partial \ln p_{a}}{\partial r}-\frac{Z_{a}}{Z_{b}} \frac{\partial \ln p_{b}}{\partial r}\right]+\left(\alpha_{1}, g_{2^{a}}\right) \frac{\partial \ln T}{\partial r}, \\
& \frac{q_{a}}{T}=\left(\alpha_{1}, g_{2^{a}}\right)\left[\frac{\partial \ln p_{a}}{\partial r}-\frac{Z_{a}}{Z_{b}} \frac{\partial \ln p_{b}}{\partial r}\right]+\left(\alpha_{2}, g_{2^{a}}\right) \frac{\partial \ln T}{\partial r}, \\
& q_{b}=\left(\beta_{2}, g_{2 b}\right) \frac{\partial T}{\partial r}
\end{aligned}
$$

which agree with the expressions given by Rutherford (1974). For the inner products, Eqs. (5.151)-(5.153) are to be used; the numerical values of the $\kappa$ 's given in Table II have been fitted to functions of $\alpha$, with the results (Rutherford, 1974)

$$
\begin{aligned}
& \frac{\kappa_{22}}{\kappa}=0.47+\frac{0.35}{0.66+\alpha}, \\
& \frac{5}{2} \frac{\kappa_{12}}{\kappa}=0.30+\frac{0.41}{0.58+\alpha}, \\
& \frac{25}{4} \frac{\kappa_{11}}{\kappa}=\frac{1.13}{\alpha}+0.50+\frac{0.56}{0.56+\alpha} .
\end{aligned}
$$

If both ion species are in the banana-plateau regime, Eqs. (6.138)-(6.140) may be simplified by the use of Eq. (6.52), and we have

$$
\begin{aligned}
\Gamma_{a}= & \frac{\left(\alpha_{1}, g_{1} a\right)}{F}\left[\frac{\partial \ln p_{a}}{\partial r}-\frac{Z_{a}}{Z_{b}} \frac{\partial \ln p_{b}}{\partial r}+\frac{Z_{a}}{Z_{b}}\left(\beta_{1}, g_{2 b}\right) \frac{\partial \ln T}{\partial r}\right] \\
& +\frac{\left(\alpha_{1}, g_{2 a}\right)}{F} \frac{\partial \ln T}{\partial r}, \\
\frac{q_{a}}{T}= & \frac{\left(\alpha_{1}, g_{2 a}\right)}{F}\left[\frac{\partial \ln p_{a}}{\partial r}-\frac{Z_{a}}{Z_{b}} \frac{\partial \ln p_{b}}{\partial r}+\frac{Z_{a}}{Z_{b}}\left(\beta_{1}, g_{2 b}\right) \frac{\partial \ln T}{\partial r}\right] \\
& +\left[\left(\alpha_{2}, g_{2 a}\right)-\frac{\left(\alpha_{1}, g_{2 a}\right)^{2}}{\left(\alpha_{1}, g_{1 a}\right)} \frac{(F-1)}{F}\right] \frac{\partial \ln T}{\partial r}, \\
q_{b}= & \left(\beta_{2}, g_{2 b}\right) \frac{\partial T}{\partial r},
\end{aligned}
$$

where only the larger, $O\left(m_{b} / m_{a}\right)^{1 / 2}$ term has been retained in $q_{b}$. These expressions agree with those given by Hinton and Moore (1974), when the proper identification of coefficients is made. For the required inner products, it is convenient to use the approximate fits obtained by Hinton and Moore (1974), in which the results are expressed as continuous functions of $\alpha$. We first define coefficients $K_{m n}^{a}$ and $K_{m n}^{b}$, as follows:

$$
\begin{aligned}
& \left(\alpha_{1}, g_{1 a}\right)=-\frac{n_{a} \rho_{a \theta}^{2} \epsilon^{1 / 2}}{\tau_{a b}} K_{11}^{a}, \\
& \left(\alpha_{1}, g_{2 a}\right)=-\frac{n_{a} \rho_{a \theta}^{2} \epsilon^{1 / 2}}{\tau_{a b}}\left(K_{12}^{a}-\frac{5}{2} K_{11}^{a}\right), \\
& \left(\alpha_{2}, g_{2 a}\right)=-\frac{n_{a} \rho_{a \theta}^{2} \epsilon^{1 / 2}}{\tau_{a b}}\left(K_{22}^{a}-5 K_{12}^{a}+\frac{25}{4} K_{11}^{a}\right), \\
& \left(\beta_{1}, g_{2 b}\right)=5 / 2-K_{12}^{b} / K_{11}^{b}, \\
& \left(\beta_{2}, g_{2 b}\right)=-\frac{n_{b} \rho_{b \theta}^{2} \epsilon^{1 / 2}}{\tau_{b b}}\left[K_{22}^{b}-\left(K_{12}^{b}\right)^{2} / K_{11}^{b}\right] .
\end{aligned}
$$

With the momentum-conserving pitch-angle scattering model which was used by Hinton and Moore (1974) to calculate the frictional coupling between the two ion species, the value of $F$, which appears in Eqs. (6.150) 
and (6.151), was found to be

$$
F=1+\left(2 m_{a} / m_{b}\right)^{1 / 2} K_{11}^{a} / \alpha K_{11}^{b} .
$$

The coefficients are given approximately as follows:

$$
\begin{aligned}
K_{11}^{a} & =\frac{0.73(1+0.53 / \alpha)}{1+1.26 \nu_{* a}(1+0.53 / \alpha)}, \\
K_{12}^{a} & =\frac{0.73(1+0.71 / \alpha)}{1+0.37 \nu_{* a}(1+0.71 / \alpha)}, \\
K_{22}^{a} & =\frac{1.46(1+0.8 / \alpha)}{1+0.2 \nu_{* a}(1+0.8 / \alpha)}, \\
K_{11}^{b} & =\frac{0.55}{1+0.94 \nu_{* b}}, \quad K_{12}^{b}=\frac{0.73}{1+0.37 \nu_{* b}}, \\
K_{22}^{b} & =\frac{1.65}{1+0.23 \nu_{* b}},
\end{aligned}
$$

where

$$
\nu_{* a} \equiv \sqrt{2} r B_{0} /\left(B_{\mathrm{po}} v_{\mathrm{th} a} \tau_{a b} \epsilon^{3 / 2}\right),
$$

and

$$
\nu_{* b} \equiv \sqrt{2} r B_{0} /\left(B_{\mathrm{po}} v_{\mathrm{thb}} \tau_{b b} \epsilon^{3 / 2}\right)=Z_{b}^{2} \nu_{* a} / \sqrt{2} Z_{a}^{2},
$$

with $\tau_{a b}$ and $\tau_{b b}$ given by Eqs. (6.141) and (6.142), and $v_{\text {th } a} \equiv\left(2 T / m_{a}\right)^{1 / 2}, v_{\text {thb }} \equiv\left(2 T / m_{b}\right)^{1 / 2}$. The numerical coefficients in Eqs. (6.159)-(6.162) were obtained by: (i) requiring the limits $\nu_{* a} \rightarrow 0, \nu_{* b} \rightarrow 0$ to give the exact banana regime values, and (ii) fitting the remaining parameter to numerical calculations similar to those of Hinton and Rosenbluth (1973), with the constraint that the plateau limiting values be independent of $\alpha$. The terms proportional to $1 / \alpha$ in Eqs. (6.159)-(6.161) are the contributions from like-species collisions. In Eq. (6.162), the assumption $\alpha \gg\left(m_{a} / m_{b}\right)^{1 / 2}$ was used; the effect, on the heavy species, of unlike-species collisions is thus neglected here, although it is retained in Eq. (6.158).

It is now easy to check on the weak-coupling approximation, using Eqs. (6.158), (6.159), (6.162), and (6.164). If both ion species are in the banana regime, then $F-1 \sim\left(m_{a} / m_{b}\right)^{1 / 2} / \alpha$, which is typically small [except in the uninteresting case $\alpha \lesssim\left(m_{a} / m_{b}\right)^{1 / 2}$ ] so that the weakcoupling approximation is valid. However, if the light species is in the banana regime, but the heavy species is in the plateau regime (note that $\nu_{* b} \gg \nu_{* a}$, assuming $Z_{b}^{2} \gg Z_{a}^{2}$ ) then

$$
F-1 \simeq 1.25\left(n_{a} / n_{b}\right)\left(m_{a} / m_{b}\right)^{1 / 2} \nu_{* a}(1+0.53 / \alpha) .
$$

Hence, if

$$
\nu_{* a}(1+0.53 / \alpha) \gtrsim\left(n_{b} / n_{a}\right)\left(m_{b} / m_{a}\right)^{1 / 2}
$$

the weak-coupling approximation is not valid. The right-hand side of the inequality is typically small; for example, with as much as $5 \%$ oxygen in a hydrogen plas$\mathrm{ma}$, it has the value 0.2 . If both ion species are in the plateau regime, then $F-1 \simeq\left(n_{a} / n_{b}\right)\left(m_{a} / m_{b}\right)^{1 / 2}$, which is typically large: the weak-coupling approximation is again not valid. As shown by Hinton and Moore (1974), the right-hand side of Eq. (6.165) gives the value of collisionality at which the banana-plateau transition actually takes place (i.e., where the diffusion coefficient becomes approximately independent of $\nu_{* a}$ ). This value may be much smaller than the "standard" value for this transition $\left(\nu_{* a} \sim 1\right)$, which makes the "plateau" diffusion coefficient much smaller than the standard one, by the factor $1 / F \simeq\left(n_{b} / n_{a}\right)\left(m_{b} / m_{a}\right)^{1 / 2}$.

The continuity equation, in which $\Gamma_{a}$ appears, has the form

$$
\frac{\partial n_{a}}{\partial t}+\frac{1}{r} \frac{\partial}{\partial r} r \Gamma_{a}=S_{a},
$$

where $S_{a}$ is the difference between the ionization and recombination rates which affect species $a$. Neglecting these effects, and in the absence of a temperature gradient $(\partial T / \partial r=0)$, diffusion leads to a final state $\left(\Gamma_{a}=0\right)$ in which $\partial \ln n_{a} / \partial r=\left(Z_{a} / Z_{b}\right) \partial \ln n_{b} / \partial r$ or

$$
n_{b}=\text { const. }\left(n_{a}\right)^{z_{b} / z_{a}}
$$

(Spitzer, 1952; Taylor, 1961; Braginskii, 1965). If species " $a$ " is hydrogen, and $Z_{b} \gg 1$, then the impurity species " $b$ " is much more highly concentrated in the center of the plasma $(r=0)$ than the hydrogen ions, in this final state. The effect of a temperature gradient is to increase the inward diffusion rate for impurities, if both ion species are in the collisional regime. As pointed out by Rutherford (1974), however, the coefficient $\partial T / \partial r$, in the banana regime expression for $\Gamma_{a}$, is positive, so that a normal temperature gradient $(\partial T / \partial r<0)$ causes inward diffusion of the light species, and hence outward diffusion of the impurity species, since $\Gamma_{b} \simeq-\left(Z_{a} / Z_{b}\right) \Gamma_{a}$. As the collisionality is increased in the banana-plateau transition, this temperature gradient "screening" effect changes sign (Hinton and Moore, 1974), leading to results qualitatively like those in the collisional regime.

\section{Closure of the Moment Equations}

The moment equations, discussed in Sec. II, become a closed set of equations only when the appropriate kinetic equations have been solved and the required moments have been calculated from the distribution functions. In this section, we discuss the closure problem for tokamak devices, in which there is an additional difficulty: the magnetic field is not completely determined by currents external to the plasma, but must be determined self-consistently with the transport processes in the plasma (Grad and Hogan, 1970; Grad, 1970). The goal is to obtain a set of equations which, with appropriate initial data and boundary conditions, completely determine the spatial and temporal dependence of the electron density, the electron and ion temperatures, and the magnetic field in the plasma.

As we have seen in Secs. IV-VI, the magnetic surfaces provide the basis for the coordinate system in which the transport relations - linear relations between "fluxes" and "forces" - are given. In fact, the fluxes are averages of certain odd moments over these magnetic surfaces, while the forces are derivatives of even moments with respect to a distance coordinate perpendicular to the surfaces. (Although the transport relations were not derived in precisely this form, the quantity $\left\langle E_{\|} B\right\rangle$ having been treated as a "force", we will show, shortly, how they may be put in this form.) Since the magnetic field configuration in tokamaks depends on the plasma pressure, which changes on the diffusion time scale, we need an equation to determine the time dependence of the mag- 
netic field, on this time scale.

The equation of magnetohydrodynamic equilibrium, Eq. (2.90), is usually considered to be the equation which determines the spatial dependence of $\psi$, the poloidal flux, with $P(\psi)$ and $I(\psi)$ regarded as known functions. We are concerned here with low- $\beta$ plasmas, which are evolving because of dissipation and transport processes, and possibly because of slowly changing, external, poloidal magnetic fields, in the presence of a constant external toroidal magnetic field. It is then more natural to consider the poloidal magnetic field, or $\psi$, to be determined by Faraday's law, Eq. (2.103), with an electric field consistent with the transport processes. The role of the equilibrium equation, Eq. (2.90), should be to specify the flux surface configuration, i.e., the geometry, at each instant of time. (It may also be used to determine the small perturbation in the toroidal magnetic field, due to finite plasma pressure.) The equilibrium equation thus determines the family of surfaces $\psi(\overrightarrow{\mathrm{x}})=$ const at each instant of time, but not the value of $\psi$ on each surface as a function of time.

For this reason, we have introduced (in Sec. V) an effective minor radius coordinate $\rho$, which is related to the toroidal flux by $\phi=B_{\mathrm{To}} \cdot \pi \rho^{2}$, where $B_{\mathrm{To}}$ is a constant. The value of the toroidal flux which labels a given magnetic surface, may be assigned; once $I\left(\equiv R B_{T}\right)$ is known, through Eqs. (2.46) and (2.87), i.e.,

$$
\phi=\frac{1}{2 \pi} \int d^{3} \overrightarrow{\mathrm{x}} I / R^{2}
$$

integrated over the region enclosed by the given magnetic surface. The small perturbation in $I$, from its value in the absence of the plasma, may be determined by multiplying Eq. (2.90) by $R^{-2}$ and integrating over the region enclosed by the magnetic surface. Then, by introducing the volume $V$ as an auxiliary flux surface label, and differentiating with respect to $V$, we obtain

$$
\left\langle R^{-2}\right\rangle \frac{\partial}{\partial V}\left(I^{2} / 2\right)=-4 \pi \frac{\partial P}{\partial V}-\frac{\partial \psi}{\partial V} \frac{\partial}{\partial V}\left(\varkappa \frac{\partial \psi}{\partial V}\right),
$$

where

$$
\mathcal{K} \equiv\left\langle|\vec{\nabla} V|^{2} / R^{2}\right\rangle .
$$

Now $I^{2}$ may be obtained by one further integration, using the boundary conditions.

Having shown how the magnetic surfaces may be labeled by toroidal flux $\phi$, or, equivalently by the effective minor radius coordinate $\rho$, we must turn to the derivation of an equation for the poloidal flux, $\psi$. First, however, we shall derive a theorem which will be needed in the following.

This theorem gives the time derivative of an integral $J$, defined by

$$
J(\Psi, t)=\int d^{3} \overrightarrow{\mathrm{x}} F(\overrightarrow{\mathrm{x}}, t)
$$

where the integral is carried out over the region interior to a moving surface $\Psi(\overrightarrow{\mathrm{x}}, t)=$ const. The result is

$$
\frac{\partial J}{\partial t}=\int d^{3} \overrightarrow{\mathbf{x}} \frac{\partial F}{\partial t}+\oint d \overrightarrow{\mathrm{S}} \cdot \overrightarrow{\mathrm{u}}_{\Psi} F,
$$

where the surface integral is taken over the surface $\Psi$ = const, and where $\overrightarrow{\mathrm{u}}_{\Psi}$ is the velocity of the constant $\Psi$ surface, defined by

$$
\frac{\partial \Psi}{\partial t}+\overrightarrow{\mathbf{u}}_{\Psi} \cdot \vec{\nabla} \Psi=0 \text {. }
$$

Note that the time derivative of $J$ is taken at constant $\Psi$, while the time derivative of $F$ is taken at constant $\vec{x}$. This theorem is easily demonstrated by writing the time derivatives as limits, of differences between values at times which differ by $\Delta t$, divided by $\Delta t$. By using $\overrightarrow{\mathrm{d}} S$ $=(d S /|\vec{\nabla} \Psi|) \vec{\nabla} \Psi$, we may write the above result as

$$
\frac{\partial}{\partial t} \int_{\Psi=\text { const }} d^{3} \overrightarrow{\mathrm{x}} F(\overrightarrow{\mathrm{x}}, t)=\int d^{3} \overrightarrow{\mathrm{x}} \frac{\partial F}{\partial t}+\oint \frac{d S}{|\vec{\nabla} \Psi|} F \overrightarrow{\mathrm{u}}_{\Psi} \cdot \vec{\nabla} \Psi .
$$

\section{A. Faraday's law}

We shall now derive an equation for $(\partial \psi / \partial t)_{\phi}$, the time rate of change of the poloidal flux associated with a surface labeled by a given value of toroidal flux. To do so, we first note that

$$
\left.\left.\left.\frac{\partial \phi}{\partial t}\right)_{\psi}+\frac{\partial \phi}{\partial \psi} \frac{\partial \psi}{\partial t}\right)_{\phi}=\frac{\partial \phi}{\partial t}\right)_{\phi} \equiv 0
$$

so that

$$
\left.\left.\frac{\partial \psi}{\partial t}\right)_{\phi}=-\frac{1}{2 \pi q} \frac{\partial \phi}{\partial t}\right)_{\dot{\psi}},
$$

where $q \equiv(1 / 2 \pi)(\partial \phi / \partial \psi)$ is the "safety factor" introduced in Sec. II.

Using the definition of $\phi$, Eq. (2.46), and the theorem expressed by Eq. (7.7), we have

$$
\left.2 \pi \frac{\partial \phi}{\partial t}\right)_{\psi}=\int d^{3} x \frac{\partial \overrightarrow{\mathrm{B}}}{\partial t} \cdot \vec{\nabla} \zeta+\oint \frac{d S}{|\vec{\nabla} \psi|} \overrightarrow{\mathrm{B}} \cdot \vec{\nabla} \zeta \overrightarrow{\mathbf{u}}_{\psi} \cdot \vec{\nabla} \psi,
$$

where $\overrightarrow{\mathbf{u}}_{\psi}$ is the velocity of the constant- $\psi$ surface

$$
\left.\overrightarrow{\mathrm{u}}_{\psi} \cdot \vec{\nabla} \psi \equiv-\frac{\partial \psi}{\partial t}\right)_{\overrightarrow{\mathrm{x}}}=-c R \cdot E_{T} .
$$

By multiplying Faraday's law, Eq. (2.103), by $\vec{\nabla} \zeta$,

$$
\frac{\partial \overrightarrow{\mathrm{B}}}{\partial t} \cdot \vec{\nabla} \zeta=-c \vec{\nabla} \cdot(\overrightarrow{\mathrm{E}} \times \vec{\nabla} \zeta),
$$

integrating over the region enclosed by the surface $\psi$ = const., and using the divergence theorem, we obtain

$$
\int d^{3} \overrightarrow{\mathrm{x}} \frac{\partial \overrightarrow{\mathrm{B}}}{\partial t} \cdot \vec{\nabla} \zeta=-c \oint \frac{d S}{|\vec{\nabla} \psi|} \overrightarrow{\mathrm{E}} \cdot \overrightarrow{\mathrm{B}}_{p} .
$$

Combining Eqs. (7.9)-(7.11) gives, therefore,

$$
\begin{aligned}
\left.2 \pi \frac{\partial \phi}{\partial t}\right)_{\psi} & =-c \oint \frac{d S}{|\vec{\nabla} \psi|} \overrightarrow{\mathrm{E}} \cdot \overrightarrow{\mathrm{B}}, \\
& =-c \frac{\partial V}{\partial \psi}\left\langle E_{\|} B\right\rangle,
\end{aligned}
$$

where the second equality follows from Eq. (2.56). From Eq. (7.8) we finally obtain

$$
\left.\frac{\partial \psi}{\partial t}\right)_{\phi}=\frac{c\left\langle E_{\|} B\right\rangle}{I\left\langle R^{-2}\right\rangle}
$$

where we have also used the relation

$$
q=I \frac{\partial V}{\partial \psi} \frac{\left\langle R^{-2}\right\rangle}{(2 \pi)^{2}}
$$


which follows from the definition, Eq. (2.50). The reason for using $\rho$ (which is equivalent to using $\phi$ ) as the spatial coordinate should now be clear: Eq. (7.13) is a simple and exact equation, which contains the electric field only through $\left\langle E_{\|} B\right\rangle$, which is obtainable from the transport relations.

It is actually more convenient, in applications, to use an expression for the time derivative of the effective poloidal field $B_{\mathfrak{y}}(\rho, t)$, defined by Eq. (5.36). To obtain this expression, we simply differentiate Eq. (7.13) with respect to $\rho$

$$
\frac{\partial B_{p o}}{\partial t}=c \frac{\partial E_{0}}{\partial \rho},
$$

where

$$
E_{0} \equiv\left\langle E_{\|} B\right\rangle / I R_{0}\left\langle R^{-2}\right\rangle .
$$

This equation has the form of Faraday's law for a cylindrical system, with $E_{0}$ as the axial component of the electric field.

Next, we derive a useful expression for the velocity, $\overrightarrow{\mathrm{u}}_{\rho}$, of a surface of constant $\rho$ (or constant $\phi$ ). We note that

$$
\overrightarrow{\mathrm{u}}_{\rho} \cdot \vec{\nabla} \rho \cdot=\overrightarrow{\mathrm{u}}_{\rho} \cdot \vec{\nabla} \phi / \frac{\partial \phi}{\partial \rho}=\overrightarrow{\mathrm{u}}_{\phi} \cdot \vec{\nabla} \phi / \frac{\partial \phi}{\partial \rho},
$$

where

$$
\overrightarrow{\mathrm{u}}_{\phi} \cdot \vec{\nabla} \phi \equiv-\frac{\partial \phi}{\partial t},
$$

and the time derivative is taken at constant $\overrightarrow{\mathrm{x}}$. Also, since

$$
\left.\left.\left.\frac{\partial \psi}{\partial t}\right)_{\overrightarrow{\mathbf{x}}}=\frac{\partial \psi}{\partial t}\right)_{\phi}+\frac{\partial \psi}{\partial \phi} \frac{\partial \phi}{\partial t}\right)_{\overrightarrow{\mathbf{x}}},
$$

we have

$$
\left.\left.\overrightarrow{\mathbf{u}}_{\phi} \cdot \vec{\nabla} \phi=\left[\frac{\partial \psi}{\partial t}\right)_{\phi}-\frac{\partial \psi}{\partial t}\right)_{\overrightarrow{\mathbf{x}}}\right] / \frac{\partial \psi}{\partial \phi} .
$$

Finally, combining Eqs. (7.16), (7.18), (7.10), and (7.13), we have

$$
\overrightarrow{\mathrm{u}}_{\rho} \cdot \vec{\nabla} \rho=\left[c\left\langle E_{\|} B\right\rangle / I\left\langle R^{-2}\right\rangle-c R E_{T}\right] / R_{0} B_{\text {po }} \cdot
$$

\section{B. Ampere's law}

The transport theory described in earlier sections allows us to relate $\left\langle E_{\|} B\right\rangle$ to $\left\langle J_{\|} B\right\rangle$ (as well as to spatial derivatives of the density and temperatures). We now show how $\left\langle J_{\|} B\right\rangle$ can be expressed in terms of spatial derivatives of $\psi$. We have

$$
\left\langle J_{11} B\right\rangle=\left\langle\overrightarrow{\mathrm{J}}_{p} \cdot \overrightarrow{\mathrm{B}}_{p}\right\rangle+\left\langle\overrightarrow{\mathrm{J}}_{T} \cdot \overrightarrow{\mathrm{B}}_{T}\right\rangle
$$

where the subscripts $p$ and $T$ denote the poloidal and toroidal components, respectively. Using the poloidal and toroidal components of Ampere's law, Eq. (2.42),

$$
\begin{aligned}
& \vec{J}_{p}=\frac{c}{4 \pi} \vec{\nabla} \times \overrightarrow{\mathrm{B}}_{T}=-\frac{c}{4 \pi} \frac{\partial I}{\partial \psi} \overrightarrow{\mathrm{B}}_{p}, \\
& \overrightarrow{\mathrm{J}}_{T} \cdot \vec{\nabla} \zeta=\frac{c}{4 \pi} \vec{\nabla} \times \overrightarrow{\mathrm{B}}_{p} \cdot \vec{\nabla} \zeta=\frac{c}{4 \pi} \vec{\nabla} \cdot\left(\vec{\nabla} \psi / R^{2}\right),
\end{aligned}
$$

we have

$$
\left\langle J_{\|} B\right\rangle=-\frac{c}{4 \pi} \frac{\partial I}{\partial \psi}\left\langle B_{p}^{2}\right\rangle+\frac{c}{4 \pi} I \frac{\partial}{\partial V}\left[\frac{\partial V}{\partial \psi}\left\langle B_{p}^{2}\right\rangle\right],
$$

which may be written as

$$
\left\langle J_{\|} B\right\rangle=\frac{c}{4 \pi} I^{2} \frac{\partial}{\partial V}\left[\frac{\varkappa}{I} \frac{\partial \psi}{\partial V}\right],
$$

where $\kappa$, as defined by Eq. (7.3), may also be written as

$$
\kappa=\left(\frac{\partial V}{\partial \psi}\right)^{2}\left\langle B_{p}^{2}\right\rangle .
$$

When the effective minor radius $\rho$ is used as the spatial coordinate, we have

$$
\left\langle J_{\|} B\right\rangle=\frac{c I^{2}}{4 \pi V^{\prime}(\rho)} \frac{\partial}{\partial \rho}\left[\frac{\varkappa}{I V^{\prime}(\rho)} \frac{\partial \psi}{\partial \rho}\right],
$$

where $V^{\prime}(\rho) \equiv \partial V / \partial \rho$, or

$$
\left\langle J_{\|} B\right\rangle=\frac{c I^{2}}{4 \pi V^{\prime}(\rho)} \frac{\partial}{\partial \rho}\left[\frac{\varkappa R_{0}}{I V^{\prime}(\rho)} B_{\mathrm{po}}\right],
$$

where $B_{\text {po }}$ is the effective poloidal field, defined by Eq. (5.36).

\section{Transport relations}

We now wish to make two points, with regard to the relationships expressed by Eqs. (5.70)-(5.73). The first is quite simple: although the electric field appears in the role of a force, through $A_{3 e} \equiv\left\langle E_{\|} B\right\rangle B_{0} /\left\langle B^{2}\right\rangle$, and the current density appears as a flux, these roles may easily be interchanged. That is, by solving Eq. (5.72) for $A_{3 e}$, in terms of $\left\langle J_{11} / h\right\rangle$ and $A_{1 e}, A_{2 e}, A_{4 e}$, and substituting the result into Eqs. (5.70) and (5.71), we obtain transport relations in which $\left\langle E_{11} B\right\rangle$ appears as a flux, and $\left\langle J_{\|} / h\right\rangle\left(=\left\langle J_{11} B\right\rangle / B_{0}\right)$ appears as a force. Note that the Spitzer current term in Eq. (5.72) is, by definition

$$
\left\langle J_{\| s} / h\right\rangle=\sigma_{\| 1}\left\langle E_{\|} B\right\rangle / B_{0} \text {. }
$$

It is natural to regard $\left\langle J_{H} B\right\rangle$ as a force, since it is given in terms of spatial derivatives by $\mathrm{Eq}$. (7.20). It is also natural that $\left\langle E_{\|} B\right\rangle$ should be regarded as a flux: recall Eq. (2.115), which gives the Poynting flux relative to a constant- $\psi$ surface, averaged over the surface.

The second point which we wish to make is that the particle flux, relative to a constant- $\phi$ surface, is simply related to the flux $\Gamma_{e}$, defined by Eq. (5.62). [Recall that $\mathrm{Eq}$. (2.108) gives the particle flux relative to a constant- $\psi$ surface.] To find this relation, we begin with Eq. (3.81). Noting that the essential difference between (the radial components of) $\overrightarrow{\mathrm{v}}_{D}$, Eq. (3.17), and $\overrightarrow{\mathrm{v}}_{d}$, $\mathrm{Eq}$. (3.24), is the inductive $E \times B$ drift, we obtain, from Eq. (3.81),

$$
\begin{aligned}
\left\langle n_{e} \overrightarrow{\mathrm{u}}_{e} \cdot \vec{\nabla} \rho\right\rangle= & \left\langle\int d^{3} v \overrightarrow{\mathrm{v}}_{d e} \cdot \vec{\nabla} \rho f_{e}\right\rangle+\left\langle n_{e} c \frac{\overrightarrow{\mathrm{E}}^{(A)} \times \overrightarrow{\mathrm{B}} \cdot \vec{\nabla} \rho}{B^{2}}\right\rangle \\
& +\left\langle n_{e} \overrightarrow{\mathrm{u}}_{c} \cdot \vec{\nabla} \rho\right\rangle,
\end{aligned}
$$

where $\overrightarrow{\mathrm{E}}^{(A)} \equiv-c^{-1} \partial \overrightarrow{\mathrm{A}} / \partial t$ is the inductive electric field. From now on, we shall neglect the classical flux $\left\langle n \overrightarrow{\mathrm{u}}_{c} \cdot \vec{\nabla} \rho\right\rangle$, although it is easily included.

The first term on the right-hand side of Eq. (7.22) can be transformed, by using Eq. (5.42), in which the indefinite integral can be eliminated by using Eq. (3.32) and 
integrating by parts. In terms of $\Gamma_{e}$, defined by Eq. (5.62), we find

$$
\left\langle\int d^{3} v \overrightarrow{\mathrm{v}}_{d e} \cdot \vec{\nabla} \rho f_{e l}\right\rangle=\Gamma_{e}+\frac{n_{e} c B_{\mathrm{To}}}{B_{\mathrm{po}}}\left[\frac{\left\langle E_{\|} B\right\rangle}{\left\langle B^{2}\right\rangle}-\left\langle E_{\|} / B\right\rangle\right] .
$$

The second term on the right-hand side of Eq. (7.22) can be written in a different form by multiplying Eq. (2.98) by $n_{e} c /(B \partial \psi / \partial \rho)$ and averaging

$$
\left\langle\frac{n_{e} c \overrightarrow{\mathrm{E}}^{(A)} \times \overrightarrow{\mathrm{B}} \cdot \vec{\nabla} \rho}{B^{2}}\right\rangle=n_{e} c\left[I\left\langle E_{\|} / B\right\rangle-\left\langle E_{T} R\right\rangle\right] / R_{0} B_{\mathrm{p} 0} .
$$

By combining Eqs. (7.22)-(7.24), we obtain

$$
\left\langle n_{e} \overrightarrow{\mathrm{u}}_{e} \cdot \vec{\nabla} \rho\right\rangle=\Gamma_{e}+\frac{n_{e} c}{B_{\mathrm{po}}}\left[B_{\mathrm{To}} \frac{\left\langle E_{\|} B\right\rangle}{\left\langle B^{2}\right\rangle}-\frac{\left\langle E_{T} R\right\rangle}{R_{0}}\right] .
$$

Finally, using Eq. (7.19) for the velocity of a constant$\phi$ surface, we have

$$
\left\langle n_{e}\left(\overrightarrow{\mathrm{u}}_{e}-\overrightarrow{\mathrm{u}}_{\rho}\right) \cdot \vec{\nabla} \rho\right\rangle=\Gamma_{e}+\frac{n_{e} c}{B_{\mathrm{po}}}\left\langle E_{\|} B\right\rangle\left[\frac{B_{\mathrm{To}}}{\left\langle B^{2}\right\rangle}-\frac{1}{I R_{0}\left\langle R^{-2}\right\rangle}\right]
$$

or

$$
\left\langle n_{e}\left(\overrightarrow{\mathrm{u}}_{e}-\overrightarrow{\mathrm{u}}_{\rho}\right) \cdot \vec{\nabla} \rho\right\rangle \simeq \Gamma_{e},
$$

where the approximation used in $\mathrm{Eq}$. (7.27) is $B^{2} \simeq B_{T}^{2}$.

The particle flux, relative to a constant- $\phi$ surface, is needed in the particle conservation law, which will presently be derived. It depends on the electric field only through the average $\left\langle E_{\|} B\right\rangle$, which is related to spatial derivatives of $\psi$ (or $B_{\mathrm{po}}$ ) through Eq. (7.20) and the transport relations, Eqs. (5.70)-(5.73). By comparing Eqs. (7.18), (7.10), and (7.13), it is clear that the particle flux, relative to a constant- $\psi$ surface, also depends upon the electric field only through $\left\langle E_{\|} B\right\rangle$; this result is due to Bernstein (1974).

\section{Particle conservation law}

As we have stressed earlier, the motion of particles in toroidal confinement systems is not localized, in general, except in a direction perpendicular to the magnetic surfaces. We can expect, therefore, to obtain a closed set of macroscopic equations only by taking averages over the magnetic surfaces.

We begin with the law of particle conservation, Eq. (2.13). By integrating this equation over the region interior to a surface $\phi=$ const. (or $\rho=$ const.) and using the divergence theorem, we have

$$
\int d^{3} \overrightarrow{\mathrm{x}} \frac{\partial n_{e}}{\partial t}+\oint \frac{d S}{|\vec{\nabla} \phi|} \vec{\nabla} \phi \cdot\left(n_{e} \overrightarrow{\mathrm{u}}_{e}\right)=0 .
$$

Next, we use the theorem expressed by Eq. (7.7) to write this equation as

$$
\frac{\partial}{\partial t} \oint d^{3} \overrightarrow{\mathbf{x}} n_{e}+\oint \frac{d S}{|\vec{\nabla} \phi|} n_{e}\left(\overrightarrow{\mathrm{u}}_{e}-\overrightarrow{\mathrm{u}}_{\phi}\right) \cdot \vec{\nabla} \phi=0 .
$$

The time derivative is to be taken at constant $\phi$ (or constant $\rho$ ). By noting that Eq. (2.55) implies

$$
\int d^{3} \overrightarrow{\mathrm{x}} n_{e}=\int_{0}^{\rho} d \rho V^{\prime}(\rho)\left\langle n_{e}\right\rangle
$$

where $V^{\prime}(\rho) \equiv \partial V / \partial \rho$ and referring to Eq. (2.56), the definition of the surface average, we differentiate Eq. (7.29) with respect to $\rho$ and obtain

$$
\frac{\partial}{\partial t}\left[V^{\prime}(\rho)\left\langle n_{e}\right\rangle\right]+\frac{\partial}{\partial \rho} V^{\prime}(\rho)\left\langle n_{e}\left(\overrightarrow{\mathrm{u}}_{e}-\overrightarrow{\mathrm{u}}_{\rho}\right) \cdot \vec{\nabla} \rho\right\rangle=0 .
$$

Thus, the surface-averaged electron density satisfies a continuity equation, in which the particle flux, relative to the surface, is to be obtained from Eqs. (7.27) and (5.70). The electron density is approximately uniform on the surface, so $\left\langle n_{e}\right\rangle \simeq n_{e}$.

In the large aspect ratio circular cross section case, in which motion of the magnetic surfaces is neglected, we have $V^{\prime} \propto \rho \simeq r$, independent of time, and Eq. (7.30) takes the familiar form

$$
\frac{\partial n_{e}}{\partial t}+\frac{1}{r} \frac{\partial}{\partial r} r \Gamma_{e}=0
$$

which is equivalent to the equation of conservation of particles in a circular cylinder.

\section{E. Energy conservation laws}

Beginning with Eq. (2.14), we may derive flux surfaceaveraged equations expressing the conservation of energy for electrons and for ions, in a manner similar to that used for the particle conservation laws. By using collisional conservation of momentum, and energy, expressed by Eqs. (2.11) and (2.12), we may write Eq. (2.14) for electrons and ions in the form

$$
\begin{aligned}
& \frac{3}{2} \frac{\partial p_{e}}{\partial t}+\vec{\nabla} \cdot \overrightarrow{\mathrm{Q}}_{e}=-Q_{i}+\overrightarrow{\mathrm{J}} \cdot \overrightarrow{\mathrm{E}}-\overrightarrow{\mathrm{u}}_{i} \cdot\left(\overrightarrow{\mathrm{F}}_{i}+n_{i} Z_{i} e \overrightarrow{\mathrm{E}}\right), \\
& \frac{3}{2} \frac{\partial p_{i}}{\partial t}+\vec{\nabla} \cdot \overrightarrow{\mathrm{Q}}_{i}=Q_{i}+\overrightarrow{\mathrm{u}}_{i} \cdot\left(\overrightarrow{\mathrm{F}}_{i}+n_{i} Z_{I} e \overrightarrow{\mathrm{E}}\right) .
\end{aligned}
$$

By integrating these equations over the region interior to a constant- $\phi$ surface and differentiating with respect to $\rho$, we obtain [compare the derivation of $\mathrm{Eq}$. (7.30)]

$$
\begin{aligned}
& \frac{3}{2} \frac{\partial}{\partial t} {\left[V^{\prime}(\rho)\left\langle p_{e}\right\rangle\right]+\frac{\partial}{\partial \rho} V^{\prime}(\rho)\left[\left\langle\overrightarrow{\mathrm{Q}}_{e} \cdot \vec{\nabla} \rho\right\rangle-\frac{3}{2}\left\langle p_{e} \overrightarrow{\mathrm{u}}_{\rho} \cdot \vec{\nabla} \rho\right\rangle\right] } \\
&=V^{\prime}(\rho)\left\{-\left\langle Q_{i}\right\rangle+\langle\overrightarrow{\mathrm{J}} \cdot \overrightarrow{\mathrm{E}}\rangle-\left\langle\overrightarrow{\mathrm{u}}_{i} \cdot\left(\overrightarrow{\mathrm{F}}_{i}+n_{i} Z_{i} e \overrightarrow{\mathrm{E}}\right)\right\rangle\right\},
\end{aligned}
$$

and a similar equation for the ion energy. By comparing Eq. (3.85) with the definition of $q_{e}$, Eq. (5.63), and neglecting the classical and electrostatic terms in Eq. (3.85), we have

$$
\left\langle\overrightarrow{\mathrm{Q}}_{e} \cdot \vec{\nabla} \rho\right\rangle=q_{e}+\frac{5}{2} T_{e}\left\langle n_{e} \overrightarrow{\mathrm{u}}_{e} \cdot \vec{\nabla} \rho\right\rangle .
$$

We will combine terms on the left-hand side of Eq. (7.34), in order that only the particle flux relative to a constant- $\phi$ surface appears. In so doing, we obtain an extra term which may be transformed by using Eq. (7.7), with $F=1$, differentiated with respect to $\rho$

$$
\frac{\partial}{\partial t} V^{\prime}(\rho)=\frac{\partial}{\partial \rho}\left[V^{\prime}(\rho)\left\langle\overrightarrow{\mathrm{u}}_{\rho} \cdot \vec{\nabla} \rho\right\rangle\right] .
$$

From this point on, we shall use the approximations $n_{e} \simeq\left\langle n_{e}\right\rangle, p_{e} \simeq\left\langle p_{e}\right\rangle$, whenever they appear convenient.

For the energy exchange term, the small mass-ratio approximation (Braginskii, 1965) gives

$$
Q_{i}=\frac{3 m_{e}}{m_{i}} \frac{n_{e}}{\tau_{e}}\left(T_{e}-T_{i}\right),
$$

in which the average is not needed, since $n_{e}, T_{e}$, and $T_{i}$ may be regarded as uniform on a magnetic surface. 
The Ohmic heating term in Eq. (7.34) can be transformed to a more useful form by using Eqs. (2.69) and $(2.89)$

$$
\vec{J} \cdot \overrightarrow{\mathrm{E}}=-(c / 4 \pi)(\partial I / \partial \psi) E_{\|} B-c(\partial P / \partial \psi) E_{T} R .
$$

The derivative $\partial I / \partial \psi$ may be eliminated by using Eq. (2.90), in which the right-hand side is $(4 \pi / c) R^{2} \vec{J} \cdot \vec{\nabla} \zeta$; multiplying by $R^{-2}$ and averaging over a magnetic surface yields

$$
-(c / 4 \pi) \frac{\partial I}{\partial \psi}=\frac{\langle\vec{\jmath} \cdot \vec{\nabla} \zeta\rangle+c \partial P / \partial \psi}{I\left\langle R^{-2}\right\rangle} .
$$

By manipulations similar to those which lead to Eq. (7.20), we may write the toroidal current term as

$$
\langle\vec{J} \cdot \vec{\nabla} \zeta\rangle=\left[c / 4 \pi V^{\prime}(\rho)\right] \frac{\partial}{\partial \rho}\left[\frac{\Re R_{0}}{V^{\prime}(\rho)} B_{\mathrm{po}}\right],
$$

where $\mathscr{K}$ is defined by Eq. (7.3). By averaging Eq. (7.38) and using Eq. (7.19) to eliminate $\left\langle E_{T} R\right\rangle$, we find

$$
\langle\overrightarrow{\mathrm{J}} \cdot \overrightarrow{\mathrm{E}}\rangle=\langle\overrightarrow{\mathrm{J}} \cdot \vec{\nabla} \zeta\rangle\left\langle E_{\|} B\right\rangle / I\left\langle R^{-2}\right\rangle+\left\langle\overrightarrow{\mathrm{u}}_{\rho} \cdot \vec{\nabla} \rho\right\rangle \partial P / \partial \rho .
$$

The Ohmic heating term is thus related to the relative Poynting flux [recall Eq. (2.115)] and to the work done by the moving surface against the plasma pressure gradient.

The remaining terms on the right-hand side of Eq. (7.34), involving $\vec{u}_{i}$, are evaluated as follows. Noting that only the $O\left(\delta^{2}\right)$ terms are required, and making explicit the $\delta$-ordering subscript, we have

$$
\begin{aligned}
\left\langle\overrightarrow{\mathrm{u}}_{i} \cdot\left(\overrightarrow{\mathrm{F}}_{i}+n_{i} Z_{i} e \overrightarrow{\mathrm{E}}\right)\right\rangle= & -Z_{i} e \partial \Phi / \partial \rho\left\langle\left(n_{i} \overrightarrow{\mathrm{u}}_{i}\right)_{2} \cdot \vec{\nabla} \rho\right\rangle \\
& +\left\langle\overrightarrow{\mathrm{u}}_{i 1} \cdot\left(\overrightarrow{\mathrm{F}}_{i}+n_{i} Z_{i} e \overrightarrow{\mathrm{E}}\right)\right\rangle .
\end{aligned}
$$

We computed $\overrightarrow{\mathrm{u}}_{i 1}$ in Sec. II. C.2; the result may be written as

$$
\overrightarrow{\mathrm{u}}_{i 1}=\hat{K}_{i} \overrightarrow{\mathrm{B}} / n_{i}-\left(c / Z_{i} e n_{i}\right)\left(\partial p_{i} / \partial \psi+Z_{i} e n_{i} \partial \Phi / \partial \psi\right) R^{2} \vec{\nabla} \zeta
$$

for the axisymmetric case [recall Eqs. (2.65), (2.66), and (2.86)]. Combining these two equations, recalling the relation between $\hat{K}_{i}$ and $\mu_{i}$, implied by Eq. (5.46), and using Eq. (2.93), we find

$$
\left\langle\overrightarrow{\mathrm{u}}_{i} \cdot\left(\overrightarrow{\mathrm{F}}_{i}+n_{i} Z_{i} e \overrightarrow{\mathrm{E}}\right)\right\rangle=\left\langle\overrightarrow{\mathrm{u}}_{i} \circ \vec{\nabla} \rho\right\rangle \partial p_{i} / \partial \rho-\mu_{i} T_{i} A_{1 i},
$$

where $A_{1 i}$ is defined by Eq. (5.59). Note that the radial electric field does not appear in Eq. (7.42); there is an electrostatic energy change due to radial diffusion, but this is precisely cancelled by the energy change due to equilibrium rotation. It follows in particular that the radial electric field never appears in the closed set of second-order moment equations.

The right-hand side of Eq. (7.42) appears in both electron and ion energy equations, with opposite signs in each equation, and represents a transfer of energy between the species. It can be simplified in either of two limiting cases:

(i) In the large aspect-ratio circular cross-section case, with electrons in the banana-plateau règime, $A_{1 i}$ is given by Eq. (6.55), in terms of the particle flux. If the ions are also in the banana-plateau regime, then $\mu_{i}$ is given by Eq. (6.50), in terms of the ion temperature gradient and the parameter $y$. Neglecting motion of the magnetic surfaces, $\left\langle\vec{u}_{i} \cdot \vec{\nabla} \rho\right\rangle$ is given by Eq. (7.27) divided by $n_{e}$, so that Eq. (7.42) reduces to

$$
\left\langle\overrightarrow{\mathrm{u}}_{i} \cdot\left(\overrightarrow{\mathrm{F}}_{i}+n_{i} Z_{i} e \overrightarrow{\mathrm{E}}\right)\right\rangle \simeq\left[\frac{\partial p_{i}}{\partial r}+\left(y-\frac{5}{2}\right) n_{i} \frac{\partial T_{i}}{\partial r}\right] \Gamma_{e} / n_{e}
$$

This expression was first obtained by Hinton and Rosenbluth (1973); the banana regime limit, in which $y=1.33$, was given by Rosenbluth, et al., (1972).

(ii) In the collisional regime, $A_{1 i}$, as given by Eq. (5.85), is negligibly small [cf. Eq. (5.156) and the subsequent discussion].

We now collect results, and write the general surfaceaveraged electron energy equation as

$$
\begin{aligned}
\frac{3}{2} \frac{\partial}{\partial t}\left[V^{\prime}(\rho) p_{e}\right]+ & p_{e} \frac{\partial V^{\prime}}{\partial t}+\frac{\partial}{\partial \rho} V^{\prime}(\rho)\left[q_{e}+\frac{5}{2} T_{e}\left\langle n_{e}\left(\overrightarrow{\mathrm{u}}_{e}-\overrightarrow{\mathrm{u}}_{\rho}\right) \cdot \vec{\nabla} \rho\right\rangle\right] \\
=V^{\prime}(\rho)\{ & -Q_{i}+\langle\overrightarrow{\mathrm{J}} \cdot \vec{\nabla} \zeta\rangle\left\langle E_{\|} B\right\rangle / I\left\langle R^{-2}\right\rangle-\left\langle\left(\overrightarrow{\mathrm{u}}_{i}-\overrightarrow{\mathrm{u}}_{\rho}\right) \cdot \vec{\nabla} \rho\right\rangle \frac{\partial p_{i}}{\partial \rho} \\
& \left.+\mu_{i} T_{i} A_{1 i}\right\},
\end{aligned}
$$

where $Q_{i}$ is given by Eq. (7.37), and $\langle\vec{J} \cdot \nabla \zeta\rangle$ is given by $\mathrm{Eq} \cdot(7.40)$. The ion equation is very similar, the differences in the right-hand sides being an overall sign change and the absence of the $\left\langle E_{\|} B\right\rangle$ term.

In the large aspect-ratio, circular cross section case, with magnetic surface motion neglected, Eq. (7.44) takes a more familiar form

$$
\begin{aligned}
& \frac{3}{2} \frac{\partial p_{e}}{\partial t}+\frac{1}{r} \frac{\partial}{\partial r} r\left[q_{e}+\frac{5}{2} T_{e} \Gamma_{e}\right] \\
& =-Q_{i}+\frac{c E_{0}}{4 \pi r} \frac{\partial}{\partial r}\left(r B_{p_{0}}\right)-\frac{\Gamma_{e}}{n_{e}} \frac{\partial p_{i}}{\partial r}+\left(\beta_{1}, g_{2 i}\right) A_{1 i} \frac{\partial T_{i}}{\partial r},
\end{aligned}
$$

where $E_{0}$ is given by Eq. (7.15), $\left(\beta_{1}, g_{2 i}\right)$ is given by Eq. (6.136), and $A_{1 i}$ is given by Eq. (6.132).

In Eq. (7.44), the two time-derivative terms can be combined, and the equation written as follows

$$
\begin{aligned}
\frac{3}{2} \frac{\partial}{\partial t} & {\left[p_{e}\left(V^{\prime}\right)^{5 / 3}\right]+\left(V^{\prime}\right)^{2 / 3} \frac{\partial}{\partial \rho} V^{\prime}\left[q_{e}+\frac{5}{2} T_{e} \Gamma_{e}\right] } \\
& =\left(V^{\prime}\right)^{5 / 3}\left\{-Q_{i}+\langle\vec{J} \cdot \vec{\nabla} \zeta\rangle\left\langle E_{\|} B\right\rangle / I\left\langle R^{-2}\right\rangle-\frac{\Gamma_{e}}{n_{e}} \frac{\partial p_{i}}{\partial \rho}+\mu_{i} T_{i} A_{1 i}\right\} .
\end{aligned}
$$

The ion energy equation can be written in a similar form

$$
\begin{aligned}
\frac{3}{2} \frac{\partial}{\partial t}\left[p_{i}\left(V^{\prime}\right)^{5 / 3}\right]+ & \left(V^{\prime}\right)^{2 / 3} \frac{\partial}{\partial \rho} V^{\prime}\left[q_{i}+\frac{5}{2} T_{i} \Gamma_{i}\right] \\
& =\left(V^{\prime}\right)^{5 / 3}\left\{Q_{i}+\frac{\Gamma_{e}}{n_{e}} \frac{\partial p_{i}}{\partial \rho}-\mu_{i} T_{i} A_{1 i}\right\} .
\end{aligned}
$$

The meaning of the factor $\left(V^{\prime}\right)^{5 / 3}$ in the time derivatives can be made clear by considering an example in which transport processes are negligible.

\section{F. Adiabatic compression}

One successful method of heating tokamak plasmas has been compression in major radius ( $\mathrm{Bol}$, et al., 1972). By changing the external poloidal magnetic field, with a time-independent toroidal field, the equilibrium position of the plasma column can be shifted to smaller values of major radius. If this is done fast enough that dissipation and transport effects are negligible, but slowly enough that magnetohydrodynamic equilibrium is approximately maintained, the plasma is adiabatically 
compressed. In this case, we assume that the time derivative terms dominate in Eqs. (7.13), (7.30), (7.46), and (7.47), which become

$$
\begin{aligned}
& \left.\frac{\partial \psi}{\partial t}\right)_{\phi}=0, \\
& \frac{\partial}{\partial t}\left(n_{e} V^{\prime}\right)=0, \\
& \frac{\partial}{\partial t}\left[p_{e}\left(V^{\prime}\right)^{5 / 3}\right]=\frac{\partial}{\partial t}\left[p_{i}\left(V^{\prime}\right)^{5 / 3}\right]=0 .
\end{aligned}
$$

If the temperature equilibration occurs more quickly than the compression, Eq. (7.50) should be replaced by

$$
\frac{\partial}{\partial t}\left[P\left(V^{\prime}\right)^{5 / 3}\right]=0
$$

where $P \equiv p_{e}+p_{i}=\left(n_{e}+n_{i}\right) T$. Equation (7.48) states that the poloidal flux is conserved, following a surface of constant toroidal flux. By differentiating with respect to $\phi$ and using the definition of the safety factor $q, \mathbf{E q}$. (2.50), we have $q=$ const., following the flux surface motion. Equation (7.49) gives $n_{e} V^{\prime}=$ const. which expresses the conservation of the number of particles between two nearby magnetic surfaces, while Eq. (7.51) yields $P\left(V^{\prime}\right)^{5 / 3}=$ const. which is the law for the change in pressure, in an adiabatic compression. By differentiating Eq. (7.1) with respect to $V$, using Eq. (2.55), and using the definition of $\rho, \phi=B_{\mathrm{To}} \pi \rho^{2}$, we have

$$
V^{\prime}=(2 \pi)^{2} \rho B_{\mathrm{To}} / I\left\langle R^{-2}\right\rangle .
$$

Hence, the adiabatic scaling laws for rotational transform $2 \pi / q$, density, and pressure are

$$
q=\mathrm{const}, \quad n_{e} \propto\left\langle R^{-2}\right\rangle, P \propto\left\langle R^{-2}\right\rangle^{5 / 3},
$$

following a magnetic surface. These generalize somewhat the large aspect-ratio scaling laws (Furth and Yoshikawa, 1970) for which $\left\langle R^{-2}\right\rangle \simeq R_{0}^{-2}$, and $\rho$ is the initial minor radius.

\section{G. Summary}

The equations which must be solved, to determine the radial profiles of density, electron and ion temperature, and poloidal magnetic field, as functions of time, are Eqs. (7.14), (7.30), (7.46), and (7.47). By "radial profiles," we mean the dependence on the effective minor radius coordinate $\rho$ (equivalent to toroidal flux $\phi$ ). The magnetic surface configuration is to be determined, at each instant of time by Eq. (2.90), using Eqs. (7.39) and (7.40) to express $\partial I / \partial \psi$ in terms of spatial derivatives of the pressure $P$ and the poloidal magnetic field $B_{\text {po }}$. These equations describe the radial profiles and magnetic surfaces on time scales long compared with the time needed to establish magnetohydrodynamic equilibrium. We have shown that, when the transport is negligible, the equations are consistent with the scaling laws for adiabatic compression.

We have shown that these equations are a closed set of equations when the following quantities are related to the density, temperatures and poloidal field and their spatial derivatives: $\Gamma_{e}, q_{e}, q_{i},\left\langle E_{11} B\right\rangle, \mu_{i}, A_{1 i}$. But these relations are given by Eqs. (5.70)-(5.73), (5.75), and (5.76), in terms of transport coefficients ch must be obtained by first solving the kinetic equations, Eqs. $(5.80)$ and (5.81). Thus, the closure problem is solved in principle, although explicit results for the transport coefficients are known only in special cases.

The most useful special case is the large aspect-ratio, circular cross-section case, for which the geometry was discussed in Sec. VI.A. Only in this case are the transport coefficients known explicitly, for all regimes of collisionality. In order to facilitate the use of these results in computer programs, we now summarize the complete set of equations and transport coefficients for the large aspect ratio, circular cross-section case. The magnetic surface configuration is assumed here to be time-independent.

The equations which (with appropriate boundary and initial data) determine $n_{e}, T_{e}, T_{i}$, and $B_{\mathrm{po}}$ as functions of $r$ and $t$ are as follows (with $p_{e} \equiv n_{e} T_{e}$ and $p_{i} \equiv n_{i} T_{i}$ ):

$$
\begin{aligned}
& \frac{\partial n_{e}}{\partial t}+\frac{1}{r} \frac{\partial}{\partial r} r \Gamma_{e}=0 \\
& \frac{3}{2} \frac{\partial p_{e}}{\partial t}+\frac{1}{r} \frac{\partial}{\partial r} r\left(q_{e}+\frac{5}{2} T_{e} \Gamma_{e}\right) \\
& =-\frac{3 m_{e}}{m_{i}} \frac{n_{e}}{\tau_{e}}\left(T_{e}-T_{i}\right)+\frac{c E_{0}}{4 \pi r} \frac{\partial}{\partial r}\left(r B_{\mathrm{po}}\right)-\frac{\Gamma_{e}}{n_{e}} \frac{\partial p_{i}}{\partial r}+\mu_{i} T_{i} A_{1 i}, \\
& \frac{3}{2} \frac{\partial p_{i}}{\partial t}+\frac{1}{r} \frac{\partial}{\partial r} r\left(q_{i}+\frac{5}{2} T_{i} \Gamma_{e} / Z_{i}\right) \\
& =3 \frac{m_{e}}{m_{i}} \frac{n_{e}}{\tau_{e}}\left(T_{e}-T_{i}\right)+\frac{\Gamma_{e}}{n_{e}} \frac{\partial p_{i}}{\partial r}-\mu_{i} T_{i} A_{1 i},
\end{aligned}
$$

$\frac{\partial B_{\text {po }}}{\partial t}=c \frac{\partial E_{0}}{\partial r}$.

In order to have a closed set of equations, we must express $\Gamma_{e}, q_{e}, q_{i}, E_{0}, \mu_{i}$, and $A_{1 i}$ in terms of $n_{e}, T_{e}, T_{i}$, and $B_{p_{0}}$ and their derivatives. This is accomplished by the use of Eqs. (6.120)-(6.123), (6.131), (6.132), and

$$
\mu_{i} \dot{T}_{i} \simeq\left(\beta_{1}, g_{2 i}\right)\left(\partial T_{i} / \partial r\right)
$$

in which the weak coupling approximation, as discussed in Sec. V.B.4, has been used. In Eq. (6.122), one must solve for $E_{0}$, using $\left\langle E_{\|} / h\right\rangle \simeq E_{0},\left\langle J_{\| s} / h\right\rangle \simeq \sigma_{\|} E_{0}$ [with $\sigma_{\|}$ given by $\mathrm{Eq} .(5.66)]$, and

$$
\left\langle J_{\|} / h\right\rangle \simeq \frac{c}{4 \pi r} \frac{\partial}{\partial r}\left(r B_{p o}\right) .
$$

The transport coefficients which appear in these equations, the $K_{m n}$ 's, $K_{2}$ and $\left(\beta_{1}, g_{2 i}\right)$, have been fitted to analytic expressions, given by Eqs. (6.125), (6.133), and (6.136), respectively.

\section{ACKNOWLEDGMENTS}

This work was supported in part by U.S. Energy Research and Development Administration through Contract AT-(40-1)-4478 and also in part by the Alfred $P$. Sloan Foundation, through a Research Fellowship held by one of the authors (F.L.H.). Figure 11 was kindly supplied by D. W. Ross.

\section{REFERENCES}

Alfven, H., 1950, Cosmical Electrodynamics (Oxford U. P., Fairlawn, N. J.).

Artsimovich, L. A., 1972, Nucl. Fusion 12, 215.

Baños, A., Jr., 1967, J. Plasma Phys. 1, 305.

Berk, H. L., and A. A. Galeev, 1967, Phys. Fluids 10, 441. 
Bernstein, I. B., 1974, Phys. Fluids 17, 574.

Bickerton, R. J., J. W. Connor, and J. B. Taylor, 1971, Nat. Phys. Sci. 229, 110.

Bol, K., R. A. Ellis, H. Eubank, H. P. Furth, R. A. Jacobsen, L. C. Johnson, E. Mazzucato, W. Stodiek, E. L. Tolnas, 1972, Phys. Rev. Lett. 29, 1495.

Braginskii, S. I., 1965, in Reviews of Plasma Physics, edited by M. A. Leontovich (Consultants Bureau, New York), Vol. 1, p. 205.

Chandrasekhar, S., 1943, Astrophys. J. 97, 255, 263.

Chapman, S., and T. G. Cowling, 1952, The Mathematical Theory of Non-Uniform Gases, (Cambridge University, London).

Chew, G. F., M. L. Goldberger, and F. E. Low, 1956, Proc. R. Soc. Lond., 236A, 112.

Cohen, R. S., L. Spitzer, Jr., and P. McR. Routly, 1950, Phys. Rev. 80, 230.

Connor, J. W., 1973, Plasma Phys. 15, 765.

Connor, J. W., R. C. Grimm, R. J. Hastie, P. M. Keeping, 1973, Nucl. Fusion 13, 211.

Connor, J. W., and R. J. Hastie, 1973, Nucl. Fusion 13, 221.

Connor, J. W., and R. J. Hastie, 1974, Phys. Fluids 17, 114.

Coppi, B., and D. J. Sigmar, 1973, Phys. Fluids 16, 1174.

Daybelge, U., 1971, Phys. Rev. Lett. 27, 916.

de Groot, S. R., and P. Mazur, 1962, Non-equilibrium Thermodynamics (North-Holland Publ. Co., Amsterdam), Chap. 4.

Dolgov-Saveliev, G. G., V. S. Mukhovatov, V. S. Strelkov, M. N. Shepelov, and N. A. Yavlinski, 1960, in Proceedings of the Fourth International Conference on Ionization Phenomena in Gases, Uppsala (North-Holland, Amsterdam), Vol. II, p. 947.

Dreicer, H., 1959, Phys. Rev. 115, 238.

Fried, B. D., 1966, in Plasma Physics in Theory and Application, edited by W. B. Kunkel (McGraw-Hill, New York), p. 49 .

Frieman, E. A., 1970, Phys. Fluids 13, 490

Furth, H. P., and M. N. Rosenbluth, 1969, in Plasma Physics and Controlled Nuclear Fusion Research (IAEA, Vienna),

Vol. 1, p. 821 .

Furth, H. P., and S. Yoshikawa, 1970, Phys. Fluids 13, 2593.

Furth, H. P., M. N. Rosenbluth, P. H. Rutherford, W. Stodiek, 1970, Phys. Fluids 13, 3020.

Galeev, A. A., 1971, Sov. Phys.-JETP 32, 752.

Galeev, A. A., and R. Z. Sagdeev, 1968, Sov. Phys.-JETP 26, 233.

Galeev, A. A., and R. Z. Sagdeev, 1971, JETP Lett. 13, 113.

Galeev, A. A., and R. Z. Sagdeev, 1975, to be published in

Advances in Plasma Physics, edited by A. Simon and W. B. Thompson (Interscience, New York), Vol. VI.

Galeev, A. A., R. Z. Sagdeev, H. P. Furth, and M. N. Rosenbluth, 1969, Phys. Rev. Lett. 22, 511.

Gibson, A., and D. W. Mason, 1969, Plasma Phys. 11, 121.

Gibson, A., and J. B. Taylor, 1967, Phys. Fluids 10, 2653.

Glasser, A. H., and W. B. Thompson, 1973, Phys. Fluids 16, 95.

Grad, H., 1967, Phys. Fluids 10, 137.

Grad, H., 1970, Actes, Congress intern. Math. 3, 105.

Grad, H., and J. Hogan, 1970, Phys. Rev. Lett. 24, 1337.

Grad, H., and H. Rubin, 1958, in Proceedings of Second International Conference on the Peaceful Uses of Atomic Energy (United Nations, Geneva), 31, 190.

Greene, J. M., and J. L. Johnson, 1961, Phys. Fluids 4, 875. Hamada, S., 1962, Nucl. Fusion 2, 23.

Hastie, R. J., J. B. Taylor, and F. A. Haas, 1967, Ann. Phys. 41, 302 .

Hazeltine, R. D., 1973, Plasma Phys. 15, 77.

Hazeltine, R. D., 1974, Phys. Fluids 17, 961.

Hazeltine, R. D., 1975, to be published in Advances in Plasma

Physics, edited by A. Simon and W. B. Thompson (Inter-

science, New York), Vol. VI.

Hazeltine, R. D., and F. L. Hinton, 1973, Phys. Fluids 16,
1883.

Hazeltine, R. D., F. L. Hinton, and M. N. Rosenbluth, 1973, Phys. Fluids 16, 1645.

Hazeltine, R. D., and M. N. Rosenbluth, Phys. Fluids 15, 2211.

Hazeltine, R. D., A. A. Ware, D. J. Sigmar, S. P. Hirshman, J. E. McCune, E. C. Crume, J. T. Hogan, and J. F. Clarke, 1974 to be published in Plasma Physics and Controlled Nuclear Fusion Research (IAEA, Vienna).

Hinton, F. L., and T. B. Moore, 1974, Nucl. Fusion 14, 639. Hinton, F. L., and C. Oberman, 1969, Nucl. Fusion 9, 319.

Hinton, F. L., and M. N. Rosenbluth, 1973, Phys. Fluids 16, 836.

Kadomtsev, B. B., and O. P. Pogutse, 1971, Nucl. Fusion 11, 67 .

Kadomtsev, B. B., and V. D. Shafranov, 1972, Nucl. Fusion Supp. (IAE A, Vienna), p. 209

Kaufman, A. N., 1960, in the Theory of Neutral and Ionized Gases, edited by C. de Witt and J. F. Detouef (Wiley, New York).

Kaufman, A. N., 1966, in Plasma Physics in Theory and Application, edited by Wulf B. Kunkel (McGraw-Hill, New York), p. 91.

Kihara, T., and O. Aono, 1971, in Kinetic Equations, edited by Richard L. Liboff and Norman Rostoker (Gordon and Breach, New York), p. 201.

Klima, R., 1965, Czech. J. Phys. B15, 473.

Kovrizhnikh, L. M., 1969, Sov. Phys.-JETP 29, 475.

Kovrizhnikh, L. M., 1970, International Atomic Energy Agency Internal Report IC/70/124 (unpublished).

Kruskal, M. D., J. L. Johnson, M. B. Gottlieb, and L. M. Goldman, 1958, Phys. Fluids 1, 421.

Kruskal, M. D., and R. M. Kulsrud, 1958, Phys. Fluids 1, 265.

Laing, E. W., S. J. Roberts, and R. T. Whipple, 1959, J. Nucl. Energy C 1, 49.

Landau, L., 1936, Phys. Z. Sowjetunion 10, 154.

Lenard, A., 1960, Ann. Phys. (N. Y.) 3, 390.

Marshak, R. F., 1941, Ann. N. Y. Acad. Sci. 41, 49.

Maschke, E. K., 1972, Plasma Phys. 14, 141.

Montgomery, D., L. Turner, and G. Joyce, 1974, Phys. Fluids $17,2201$.

Morosov, A. I., and L. S. Solov'ev, 1966, in Reviews of Plasma Physics, edited by M. A. Leontovich (Consultants Bureau, New York), p. 201.

Northrop, T. G., 1963, The Adiabatic Motion of Charged Particles (Interscience, New York).

Ohkawa, T., 1968, Kakuyugo-Kenkyu 20, 557.

Pfirsch, D., and A. Schlüter, 1962, Max-Planck-Institut Report MPI/PA/7/62 (unpublished).

Post, R. F., 1956, Rev. Mod. Phys. 28, 338.

Prigogine, I., 1961, Introduction to Thermodynamics of Irreversible Processes (Interscience Publishers, New York), 2nd edition.

Rawls, J. M., M. S. Chu, F. L. Hinton, 1975, Phys. Fluids 18, 1160 .

Robinson, B. B., and I. B. Bernstein, 1962, Ann. Phys. 18, 110.

Rosenbluth, M. N., 1973, Bull. Am. Phys. Soc. 18, 1337.

Rosenbluth, M. N., R. D. Hazeltine, and F. L. Hinton, 1972, Phys. Fluids 15, 116.

Rosenbluth, M. N., and A. N. Kaufman, 1958, Phys. Rev. 109, 1.

Rosenbluth, M. N., and C. L. Longmire, 1957, Ann. Phys. (N. Y.) 1, 120 .

Rosenbluth, M. N., W. MacDonald, and D. Judd, 1957, Phys. Rev. 107, 1.

Rosenbluth, M. N., P. H. Rutherford, J. P. Taylor, E. A. Frieman, and L. M. Kovrizhnikh, 1971, in Plasma Physics and Controlled Nuclear Fusion Research (IAEA, Vienna), Vol. 1, p. 495. 
Rutherford, P. H., 1970, Phys. Fluids 13, 482.

Rutherford, P. H., 1974, Phys. Fluids 17, 1782.

Rutherford, P. H., and E. A. Frieman, 1968, Phys. Fluids 11, 569 .

Rutherford, P. H., L. M. Kovrizhnikh, M. N. Rosenbluth, and F. L. Hinton, 1970, Phys. Rev. Lett. 25, 1090.

Sagdeev, R. Z., and A. A. Galeev, 1970, Sov. Phys.-Dokl. 14, 1198.

Shafranov, V. D., 1958, Sov. Phys.-JETP 8, 545.

Shafranov, V. D., 1965, At. Energ. 19, 120.

Shafranov, V. D., 1966, in Reviews of Plasma Physics, edited by M. A. Leontovich (Consultants Bureau, New York), Vol. 2, p. 103.

Sigmar, D. J., 1972, talk given at the Sherwood Theory Meeting, Los Alamos, March 23 (unpublished).

Sigmar, D. J., J. F. Clarke, R. V. Neidigh, and K. L. Vander Sluis, 1974, Phys. Rev. Lett. 33, 1376.

Spitzer, L., Jr., 1952, Astrophys. J. 116, 299.
Spitzer, L., Jr., 1967, Physics of Fully Ionized Gases (Interscience, New York).

Spitzer, L., Jr., and R. Härm, 1953, Phys. Rev. 89, 977.

Stix, T. H., 1973, Phys. Fluids 16, 1260.

Stringer, T. E., 1970, Phys. Fluids 13, 810

Su, C. H., and C. Oberman, 1968, Phys. Rev. Lett. 20, 427. Tamm, I. E., 1959, in Plasma Physics and the Problem of Controlled Thermonuclear Fusion (Pergamon, New York), Vol. 1, p. 35.

Taylor, J. B., 1961, Phys. Fluids 4, 1142.

Trubnikov, B. A., 1965, in Reviews of Plasma Physics, edited by M. A. Leontovich (Consultants Bureau, New York), Vol. 1, p. 105.

Tsang, K. T., and E. A. Frieman, 1975, to be published in Phys. Fluids.

Ware, A. A., 1970, Phys. Rev. Lett. 25, 916.

Ware, A. A., 1973, Nucl. Fusion 13, 793.

Wimmel, H. K., 1970, Nucl. Fusion 10, 117. 